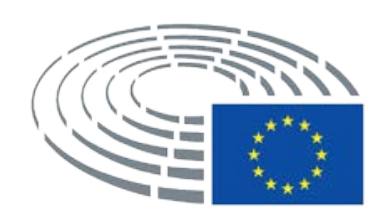

European Parliament

\section{STUDY}

Science and Technology Options Assessment

EPRS | European Parliamentary Research Service Scientific Foresight Unit (STOA)

PE 614.571
Additive biomanufacturing: 3D printing for medical recovery and human enhancement 



\title{
Additive bio-manufacturing: 3D printing for medical recovery and human enhancement
}

\author{
Study \\ IP/G/STOA/FWC/2013-001/LOT5/C2
}

July 2018

\begin{abstract}
Additive bio-manufacturing (bio-AM) is defined here as the use of 3D printing for medical purposes or non-therapeutic "human enhancement", whether they involve the production of biological material or not. It includes any application for rehabilitating, supporting or augmenting biological functionality. The impacts of bio$\mathrm{AM}$ are uncertain, and it is not clear which actions may be required to foster responsible development of the technology.

This study responds to these gaps in our knowledge by describing the state of the art and future development prospects, analysing their wide-ranging impacts - including social, ethical and economic aspects - and identifying key policy challenges along with options to respond to them. Key challenges are to avoid an incoherent, piecemeal adaptation of regulatory frameworks, to support responsible development that improves citizens' lives, and to foster public participation and citizen-driven innovation. The European RRI approach could provide a useful platform for responding to these challenges, emphasising inclusiveness and co-creation by a wide variety of stakeholder groups, including public participation.
\end{abstract}

PE 614.571 
The STOA project 'Additive bio-manufacturing: 3D printing for medical recovery and human enhancement' was requested by the European Parliament's Science and Technology Options Assessment (STOA) Panel. It was carried out by the European Technology Assessment Group (ETAG), led by the Institute for Technology Assessment and Systems Analysis at Karlsruhe Institute of Technology (KIT/ITAS) and including the Institute of Technology Assessment of the Austrian Academy of Sciences (ITA/OEAW), the Rathenau Institute, the Danish Board of Technology (DBT) Foundation and France-based Responsible Technology (RT). It was managed by the Scientific Foresight Unit (STOA) within the Directorate-General for Parliamentary Research Services (DG EPRS) of the European Parliament.

\title{
AUTHORS Riisgaard, Rasmus Øjvind Nielsen (DBT Foundation). \\ STOA ADMINISTRATOR RESPONSIBLE \\ Phillip Boucher, Scientific Foresight Unit (STOA) \\ Directorate for Impact Assessment and European Added Value \\ Directorate-General for Parliamentary Research Services \\ European Parliament, Rue Wiertz 60, B-1047 Brussels \\ E-mail: philip.boucher@europarl.europa.eu
}

Arianna Ferrari, Martina Baumann, Christopher Coenen, Daniel Frank, Leonhard Hennen, António Moniz (KIT/ITAS); Helge Torgersen, Jan Torgersen (ITA/OEAW); Lisa van Bodegom, Freija van Duijne, Ingrid Geesink, Barend van der Meulen (Rathenau Institute); Emilio Mordini (RT); Karen

Scenario illustrations by LUC - le Laboratoire d'Usages Collaboratifs - luc-lab.com

\section{Acknowledgements}

We are extremely grateful to the individuals and organisations that made important contributions to the study, including: Dario Tamburrano (MEP), Nuno Araújo, Eduard Arzt, José Barata, Thomas Bayer, Torsten Bell, Wouter Boon, Martin Malthe Borch, Alice Conquand, Carlos Cuevas-Garcia, Mark Cutkowsky, Aránzazu del Campo, Peter Dubruel, Maria Farsari, Peter Gruber, Øyvind Halaas, Michael Hasenpusch, John (J.A.) Jansen, Miet Janssen, Ali Khademhodseini, Polina Kotlarenko, Phoebe Li, Alberto Magarelli, Giorgio Magistrelli, Vladimir Mironov, Lorenzo Moroni, Iris Otto, Enno Park, Wendy Phillips, Radu Racasan, Heinz Redl, Lale Rodgarkia-Dara, Melike Şahinol, Erica Seidita, Angela Simone, Jürgen Stampfl, Coline Thomas, Aneta Tyszkiewicz, Franz Varga, Hildebrando Vasconcelos, Niki Vermeulen, Ellen Willemsen and Yu Shrike Zhang.

\section{LINGUISTIC VERSION}

\author{
Original: EN
}

\section{ABOUT THE PUBLISHER}

To contact STOA or to subscribe to its newsletter please write to: STOA@ep.europa.eu This document is available on the Internet at: http:/ / www.europarl.europa.eu/stoa/

Manuscript completed in July 2018

Brussels, (C) European Union, 2018

\section{DISCLAIMER}

This document is prepared for, and addressed to, the Members and staff of the European Parliament as background material to assist them in their parliamentary work. The content of the document is the sole responsibility of its author(s) and any opinions expressed herein should not be taken to represent an official position of the Parliament. Reproduction and translation for non-commercial purposes are authorised, provided the source is acknowledged and the European Parliament is given prior notice and sent a copy.

PE 614.571

ISBN 978-92-846-3148-3

doi: $10.2861 / 923327$

QA-01-18-636-EN-N 


\section{Table of contents}

EXECUTIVE SUMMARY 6

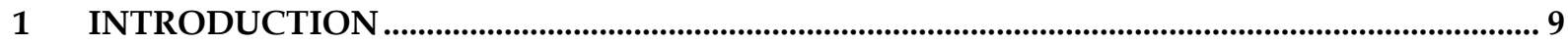

2 ADDITIVE BIO-MANUFACTURING........................................................................................... 10

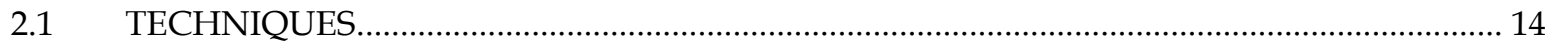

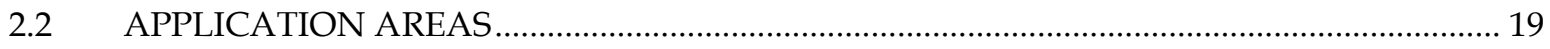

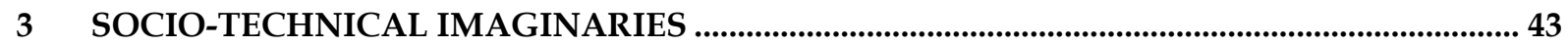

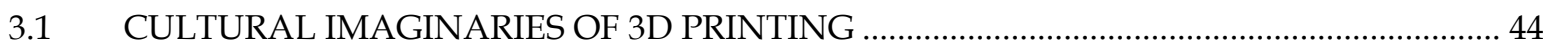

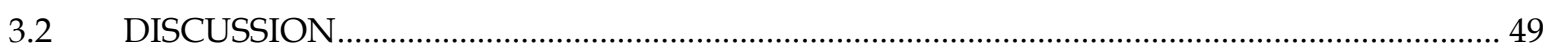

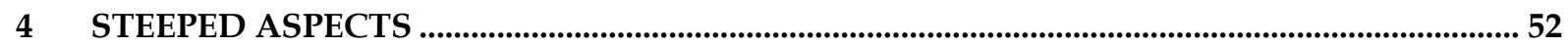

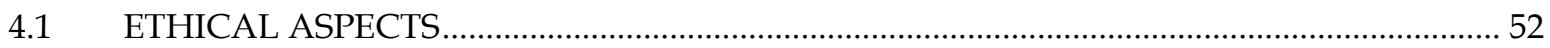

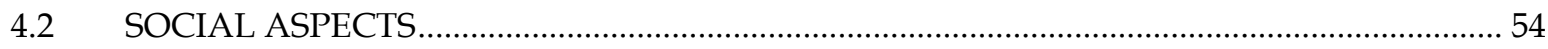

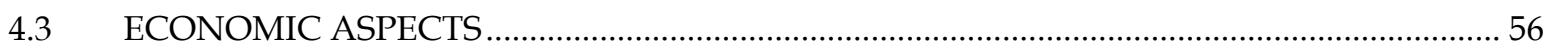

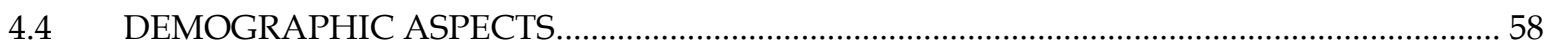

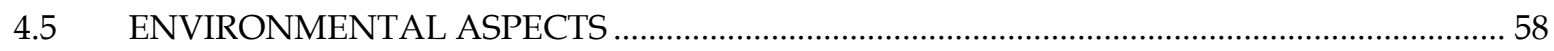

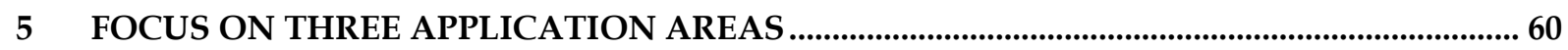

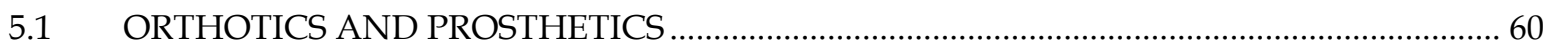

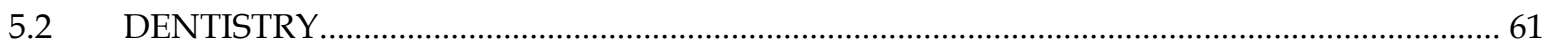

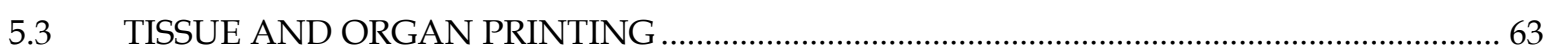

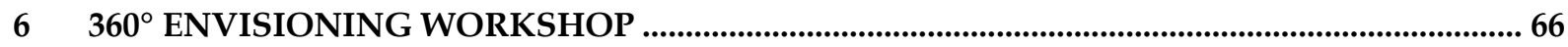

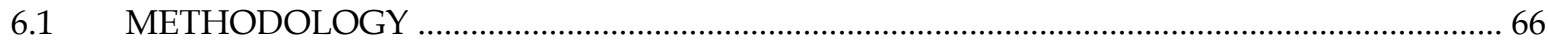

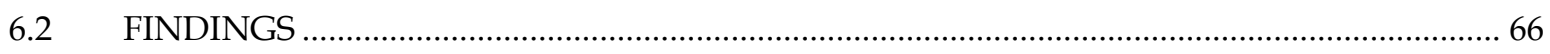

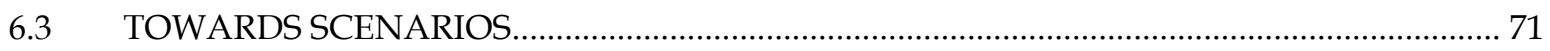

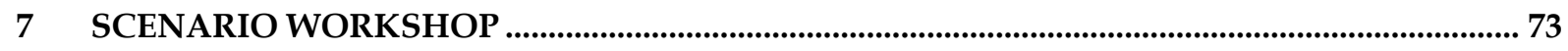

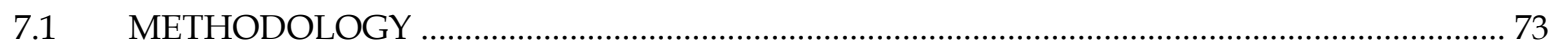

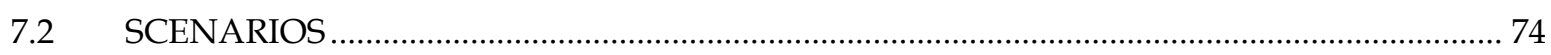

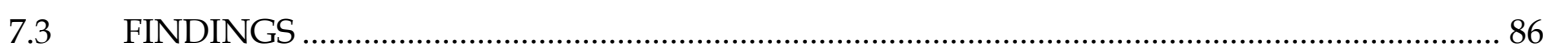

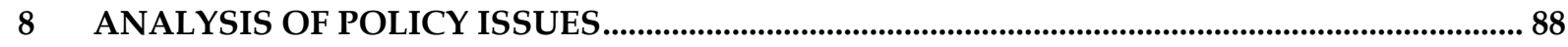

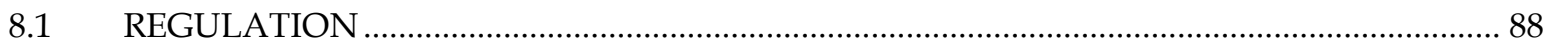

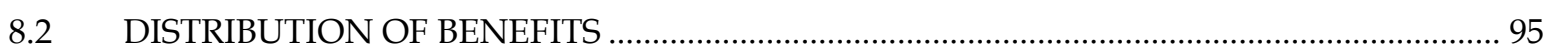

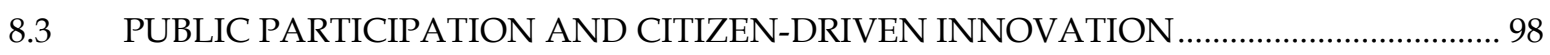

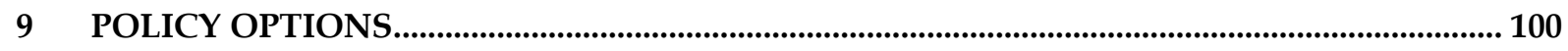

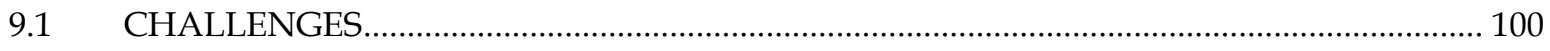

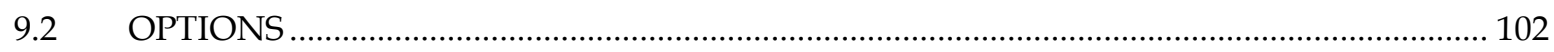

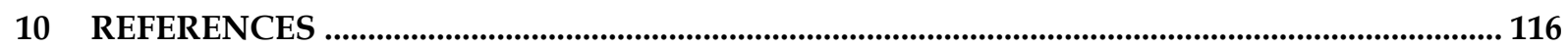




\section{Exec utive summary}

Additive manufacturing (AM, commonly known as 3D printing or rapid prototyping), allows creating parts from different kinds of materials and in complex shapes by means of computer-aided design (CAD). This means that a software is used to design a product which is then constructed layer by layer by a 3D printer. The big advantage of this technique is that parts that are only needed in small quantities or even as a single part can be produced very economically. Another feature is that products may generally be produced decentralised as only one type of manufacturing machine is needed and the blueprints for designs, the CAD files, may be distributed very easily. In the medical sector, customised products for individual needs, also called personalised treatments, promise higher efficacy and lower costs.

The project focused on additive bio-manufacturing (bio-AM), defined as the use of AM for medical purposes of for non-therapeutic "human enhancement", including all relevant AM applications whether they produce biological material or not - as long as they are deployed for the express purpose of rehabilitating, supporting or augmenting biological functionality. As bio-AM is still a very new development, the impact of this technology is uncertain in various respects: Will it revolutionise future health, does it have unintended side effects on health and, more generally, our lives, and what factors could foster or hamper innovation? And what could the European Union (EU) do to support responsible 3D printing innovation that yields benefits for industry and patients, but also for society as a whole? We thus analysed the technical state of the art and future development prospects as well as the STEEPED (Social, Technological, Environmental, Ethical, Policy, Economic and Demographic) aspects of several selected applications. The focus was on prostheses and orthoses, tissues and organs, and dental implants.

AM technology makes it possible to create precisely fitting orthoses and prostheses (with individualised designs), which may improve the situation of amputees also in developing countries, where civil society initiatives already bring 3D printed prostheses to kids. The fabrication of hearing aid shells is one of the most successful AM applications, and dental implants have the potential to become the next widely adopted 3D printing application in the medical sector. Full automation of the entire process chain is feasible, making the process faster and cheaper. Tissue engineering receives high media attention and is very promising, but today and in the near future it will mostly remain a tool for studying cell behaviour and development in 3D (such as printed liver tissue that is already used for toxicity tests).

However, progress in bio-AM does not only have an effect on the way research is done, what diseases can be treated how effectively, and how fast services like the production of dental implants can be provided. It may have numerous impacts on society, both intended and unintended, both anticipated and entirely unknown. In most cases, one can only give plausible scenarios for the impact of technology on society based on solid research on the STEEPED aspects. We did that research by reviewing literature and interviewing experts from the relevant fields of technology and research, law, ethics, and also patients. We found that there is a positive potential of 3D printing for organs to solve some problems of transplantation medicine. Bio-AM may help reduce waste, as for example in the field of food printing through the re-use of materials, the energy consumption however remains an open question. There are concerns about job losses due to changes in production methods and places, especially in specialised sectors (such as dentistry) and in developing countries. Bio-AM may contribute to the reduction of animal experiments. As regards tissue and organ printing, there is a risk of hype and subsequent disappointments which may have an impact on the acceptance of whole research areas.

In the project we also wrote techno-moral vignettes. These scenarios are stories that describe and explore plausible futures in which various bio-AM applications are used. Therein, diverse stakeholder groups, 
especially citizens and patients, are confronted with the technological possibilities of bio-AM anticipated for the next years. For example, in one scenario two teenagers want to get 3D printed tattoos, a procedure that bears safety risks but cannot be regulated by medical agencies when it is done in small Do It Yourself (DIY) labs and people cross borders to get such treatments. In this scenario, we considered two further aspects besides the STEEPED aspects: the use of AM technology for human enhancement or, more broadly speaking, non-medical uses; and the role of citizens as active players in the process of innovation, which brings also new responsibilities for citizens and challenges current ways of standardising products and ensuring safety. All scenarios show hopes and fears concerning the potential impacts of bio-AM on people's lives.

The scenarios where also designed to present and stimulate reflection on some of the identified policy issues and challenges that EU policy-makers have to anticipate and respond to. There are three broad challenges we identified.

The first is to avoid an incoherent, piecemeal adaptation of different regulatory frameworks to upcoming issues such as safety problems, but instead create a comprehensive framework of regulation that addresses the fundamental issues of bio-AM without leaving grey areas and in a timely manner. This is critical in offering safe medical treatments to patients and giving developers and manufacturers certainty about what they need to do to launch a product. Fundamental issues are the difficulty of classification of bio-AM products as advanced therapy vs. medical devices (the two broad categories that are currently used to define the tests and requirements for a specific treatment before entering the market), the need for entirely new standards for product development, validation and testing of customised, on-demand manufactured products, and questions of intellectual property rights and ownership of the data produced, e.g. when a nose of a person is used as a blueprint to 3D print a new nose for another person.

A second challenge for the EU is to support and promote the application of bio-AM technology for improving public health in a responsible way and affordable for wider society. Here, funding schemes and intellectual property rights / patenting regulation will be critical in directing research and development activities towards diseases with high societal burden and ensuring that also smaller firms have access to research data, and all people in need to the end products.

A third challenge is to foster public participation and citizen-driven innovation - two crucial aspects of responsible research and innovation (RRI) - in order to support innovation and initiate public dialogue on societal and other aspects of bio-AM. Options are numerous here and include the design and creation of a EU-wide network of citizen-innovation spaces, improved public communication of science by researchers also in order to counter tendencies of hype, and the organisation of inclusive and publicly visible deliberations on specific bio-AM applications as well as on the relevance of bio-AM in broader research, innovation and health policy contexts. The latter is crucial due to the cross-cutting and enabling character of AM technologies.

By creating evidence-based mid-term scenarios, we tried to bridge the gap between an assessment of the current state of the art and visionary prospects. However, for technologies that cross-cut a wide range of science, technology and application areas, this may never be completely achieved. Thus, we propose that a main task of European policy should be to initiate processes where the interested public can participate in shaping the future of innovation by bringing in their values and needs. The EU should take advantage of having an already well-developed, comprehensive approach to responsible innovation which puts particular emphasis on inclusiveness and the co-creation of innovation by a wide variety of stakeholder groups, including public participation. The European RRI approach could fruitfully be applied with respect to bio-AM, in order to foster new pro-innovation coalitions between 
policy actors, companies, academic institutions, other established players in the innovation system and citizens who are engaged in citizen-driven innovation initiatives, or simply want to have their say concerning the future of science and technology in European societies. 


\section{Introduction}

AM allows parts to be created in an additive manner, by means of computer-aided design (CAD). The main advantage of this technique is that small batch sizes can be achieved more economically than with other manufacturing processes. Each individual structure can be customised. In some cases, several different parts can be created in a single batch. AM systems function without user interaction, combining computer-aided design with manufacturing (CAD-CAM).

Recently, AM has become of interest and accessible to many potential users. The complex shapes obtainable, the simple operation, the ever-increasing availability and the rapid decline in prices have triggered serious excitement and a real hype for this technology. Users expect the fabrication of fully customised objects of varied types and functions, created easily and according to their personal designs. Engineers and designers are enthusiastic about applying AM in almost all sectors of industrial manufacturing, in engineering, electronics and healthcare (Chhaya et al. 2015). Especially in the healthcare sector, where low batch sizes and customisation are of high importance, AM has shown to be most effective in creating products from patients' 3D medical data constituting complex organic inputs. It has been successfully applied in the following areas: surgical, diagnostic and educational aids; prosthesis development; drug delivery; tissue engineering/organ printing (see, e.g., Norman et al. 2017). Under the umbrella term 3D bioprinting, it was in particular prosthesis and organ printing that has brought AM significant media attention. These applications seem to be only the tip of the iceberg, and many foresee much broader use of additive bio-manufacturing (bio-AM), with all kinds of potential implications.

The project explored 'additive bio-manufacturing for medicine and human enhancement' (bio-AM). Our definition of Bio-AM includes all biologically relevant additive manufacturing technologies whether they produce biological material or not - as long as they are deployed for the express purpose of rehabilitating, supporting or augmenting biological functionality. The project began by reviewing major trends in the field, before focussing on specific application areas, exploring their implications through a scenario method, identifying major policy issues and developing potential options for responding to them.

The next section provides an overview of AM in the medical sector, descriptions of available techniques and major fields of medical and non-medical application, both current and speculative (section 2). This is followed by a review of bio-AM 'imaginaries', which provide an insight into how we might make sense of the technology (section 3) and a detailed review of social, ethical, economic and other aspects of bio-AM development (section 4). The study then provides a more detailed description of applications in three particular areas: orthotics and prosthetics, dentistry, and tissue and organ printing (section 5). These applications were explored in greater depth during ' $360^{\circ}$ envisioning' and scenario workshops (described in sections 6 and 7). The final sections outline major policy issues identified in the course of the study (section 8) and describe policy options along with their possible outcomes (section 9). 


\section{Additive bio-manufacturing}

$\mathrm{AM}$ is one of the most rapidly developing fields of industrial engineering. Its potential has been dealt with in many scientific, economic and media reports. While some claims seem to be exaggerated, the significance of AM for future production in a wide variety of fields is undeniable. One of the most outstanding fields is medical technology.

However, sometimes ambiguities seem to prevail in the understanding of what AM essentially is. According to ASTM F2792-10 standard (ASTM 2010), AM is defined as "the process of joining materials to make objects from 3D model data, usually layer upon layer, as opposed to subtractive manufacturing technologies." Thus, AM allows creating parts in an additive manner by means of computer-aided design (CAD). More specifically (Gebhardt 2007), AM technologies are processes in which

- a part is built adding slices (cross sections of a part) on top of each other;

- slices are generated from CAD files that are universal to all AMTs;

- no tooling is required during fabrication;

- mechanical and topological properties evolve simultaneously;

- workpieces can be manufactured in any orientation; and

- clamping can be entirely omitted.

These properties enable the fabrication of unique structures (e.g. cellular or gyroid) within one step and without requiring process-specific know-how.

Over the last three decades, AM has undergone rapid development (Figure 1) from the first stereolithography and acrylate printing in the mid-1980s to today's advanced technologies including micro-scale two-photon polymerisation or bioprinting using living cells. Figure 1 depicts some of the most important milestones.

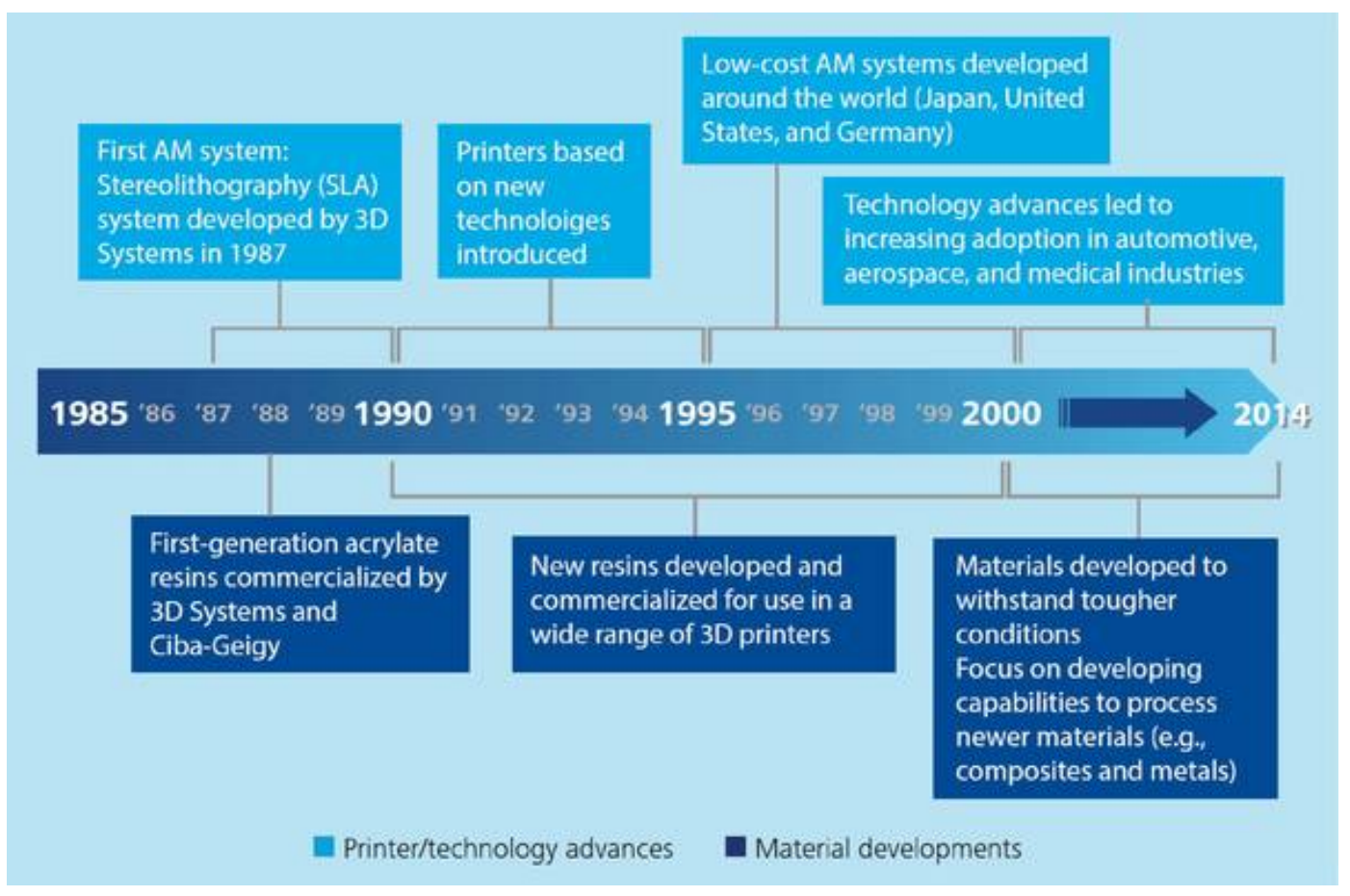

Figure 1 The milestones of additive manufacturing. Source: DU Press, Wohlers Associates http://www.cecimo.eu/site/additive-manufacturing/am-from-its-beginnings/.

Over time, AM has become interesting and accessible to many potential users. The complex shapes obtainable, the simple operation, the rapidly growing availability and the fast decline in price and the 
increasing choice of materials triggered serious excitement and, sometimes, a real hype. Users imagined the entire fabrication of fully customised objects of various kinds and functions, easily created according to their personal designs. Today, many engineers and designers are enthusiastic to apply AM in almost all sectors of industrial manufacturing, engineering, education, electronics and health care (Chhaya et al., 2015; Lipson, 2014). Figure 2 gives an overview over applications that some years ago had already been realised or were then expected to be realised. Today, a number of them have materialised indeed, while others still remain dreams of the future.

Among the many applications for additive manufacturing, the medical technology industry has been one of the sectors that take most advantage. AM is often expected, and in fact has already started, to bring about a revolution in customised medicine. Here, low batch sizes and customisation are of high importance. AM has shown to be most effective in creating products in various fields from patients' $3 \mathrm{D}$ medical data constituting complex organic inputs. Not only dental implants, prostheses, orthoses and hearing aids are manufactured to fit an individual's unique physiology, but also surgical tools, drug delivery devices as well as approaches using tissue engineering in regenerative medicine (Gibson et al. 2015; Norman et al. 2017). Thus, AM based technologies are rapidly progressing towards clinical application in various fields or have already been introduced and pave their way towards broader use. Key benefits of AM in the medical field are:

- freedom of design, complex shape, thin walls and shapes impossible to produce by casting;

- light weight parts, flexibility in design changes;

- customised design;

- no tooling ${ }^{1}$ needed;

- short production time.

Regarding materials applied, traditional medical implants have been fabricated mostly from metallic compounds such as stainless steel. A wide range of alloys can also be used based on titanium; nickel, cobalt, iron, rare earth, aluminium etc. In addition, specialised polymeric and other synthetic materials are currently being developed for implant purposes. All of them are also amenable to AM now.

Under the umbrella of biofabrication, especially tissue engineering and regenerative medicine has brought AM to significant professional as well as media attention. They have engaged scientists, engineers and clinicians to contribute pushing this field to one of the most rapidly advancing areas of biomedical science (Chhaya et al. 2015). The significant attention, the ongoing and continuous discussion about AM related topics and the prospects of its industrial use have shaped AM's image profoundly.

Nevertheless, this does not go undisputed: we can see two opposing opinions regarding the feasibility and future distribution of AM. While the speculations of visionary thinkers sketch out a future where AM has replaced most of conventional fabrication methods, conservative practitioners are reluctant to anticipate any significant role for AM in production processes apart from specialised niche applications. As a platform technology, AM certainly brings competitive advantages for producing complex, personalised and on-demand products. While AM has evident advantages, some factors still impede the full application of the technology. ${ }^{2}$ Among others:

- systems are expensive;

- the size of the objects is limited;

- necessary production time restricts mass production;

- products need finishing;

- processes need to be strictly controlled;

\footnotetext{
1 This term refers to the adaptation and adjustment of the machinery that is necessary for other forms of fabrication (e.g. casting, milling or lathing) of a work piece.

2 Giorgio Magistrelli, expert in additive manufacturing, in A3DM Magazine 20, October 2016
} 
- qualification procedures and process approbations are time-consuming;

- user communities for 3D prints, especially from metals, have not yet established.

\begin{tabular}{|c|c|c|}
\hline INDUSTRIES & CURRENT APPLICATIONS & POTENTIAL FUTURE APPLICATIONS \\
\hline $\begin{array}{l}\text { COMMERCIAL } \\
\text { AEROSPACE } \\
\text { AND } \\
\text { DEFENSE } 17\end{array}$ & $\begin{array}{l}\text { - Concept modeling and prototyping } \\
\text { - Structural and non-structural } \\
\text { production parts } \\
\text { - Low-volume replacement parts }\end{array}$ & $\begin{array}{l}\text { - Embedding additively manufactured } \\
\text { electronics directly on parts } \\
\text { - Complex engine parts } \\
\text { - Aircraft wing components } \\
\text { - Other structural aircraft components }\end{array}$ \\
\hline SPACE & $\begin{array}{l}\text { - Specialized parts for space } \\
\text { exploration } \\
\text {. Structures using light-weight, } \\
\text { high-strength materials }\end{array}$ & $\begin{array}{l}\text { - On-demand parts/spares in space } \\
\text { - Large structures directly created in } \\
\text { space, thus circumventing launch } \\
\text { vehicle size limitations }\end{array}$ \\
\hline AUTOMOTIVE ${ }^{18}$ & $\begin{array}{l}\text { - Rapid prototyping and } \\
\text { manufacturing of end-use auto parts } \\
\text { - Parts and assemblies for antique cars } \\
\text { and racecars } \\
\text { - Quick production of parts or entire }\end{array}$ & $\begin{array}{l}\text { - Sophisticated auto components } \\
\text { - Auto components designed through } \\
\text { crowdsourcing }\end{array}$ \\
\hline $\begin{array}{l}\text { HEALTH } \\
\text { (AD CARE }{ }^{19} \\
\text { aD })\end{array}$ & $\begin{array}{l}\text { - Prostheses and implants } \\
\text { - Medical instruments and models } \\
\text { - Hearing aids and dental implants }\end{array}$ & $\begin{array}{l}\text { - Developing organs for transplants } \\
\text { - Large-scale pharmaceutical } \\
\text { production } \\
\text { - Developing human tissues for } \\
\text { regenerative therapies }\end{array}$ \\
\hline $\begin{array}{l}\text { CONSUMER } \\
\text { PRODUCTS/RETAIL }\end{array}$ & $\begin{array}{l}\text { - Rapid prototyping } \\
\text { - Creating and testing design iterations } \\
\text { - Customized jeweiry and watches } \\
\text { - Limited product customization }\end{array}$ & $\begin{array}{l}\text { - Co-designing and creating with } \\
\text { customers } \\
\text { - Customized living spaces } \\
\text { - Growing mass customization of } \\
\text { consumer products }\end{array}$ \\
\hline
\end{tabular}

Figure 2 AM applications by main markets. Source: Dupress.

https://dupress.deloitte.com/dup-us-en/focus/3d-opportunity/the-3d-opportunity-primer-the-basics-ofadditive-manufacturing.html\#endnote-17

In addition, the lack of a clear definition might contribute to a somewhat skewed impression of AM's technical capabilities. The research field's definition itself carries inconsistencies and requires refinement (Groll et al. 2016). ${ }^{3}$ In the longer run, this might jeopardise the confidence in the technology, hinder its introduction for useful applications and, in effect, hamper innovation and future revenue. Research results can be made comparable and treatments applicable for medical doctors only through reliable standards. The combination of lack of oversight, media hype and missing standards (Chhaya et al. 2015; Lipson 2014) may have led to oversimplified, overpromised and exaggerated perspectives and results.

The fundamental principle of AM, namely the direct transformation of a digital file into a physical object, carries wider societal risks and challenges as well. Some might require regulatory or political action, for example the misuse of sensible patient specific data, the safeguarding of intellectual property

\footnotetext{
${ }^{3}$ For example, the terms biofabrication and bioprinting are used interchangeably and predominantly in conjunction with AM.
} 
rights of designs as well as the democratisation and access to AM related healthcare. Here, smart policies can balance incentives and rewards for investors with innovation advancing free access to designs.

In general, $\mathrm{AM}$ is said to contribute to the democratisation of production, ${ }^{4}$ distributing the production process to the end user even. This would imply that items are not centrally produced, shipped and bought at the retailer; rather, electronic data files are being sent to the local user who gets the item made in his own 3D printer. Such projections, however, meet fundamental obstacles. This also applies to AM in the context of bio- and medical applications; however, the requirements in the medical field differ from industrial production in general in some respects. Nevertheless, important conclusions can be drawn from more general considerations also for medical and/or bio-AM.

As Matthias Holweg, professor of operations management at the University of Oxford's Saïd Business School, wrote in a blog for Harvard Business Review, ${ }^{5}$ there are severe limitations to 3D printing. From economic reasons alone, printed items could not compete with centrally fabricated ones unless there is a special context such as spare requirement in a space ship or at remote places, where transport would be unfeasible.

Another problem is that functionally more complex items require special materials that are difficult to handle. Metals need to be sintered, which requires powerful laser beams that cannot be handled at home or in a small hospital. The same goes for special plastic materials that need to be cured in toxic solutions. ${ }^{6}$

Finally, skill is a limiting factor in the $3 \mathrm{D}$ printing process despite claims that it would lead to a deskilling and, hence, to a distribution of the ability to produce. At a workshop organised by the Royal Society of Engineering, "3T RPD Chief Executive Officer Dr Ian Halliday drew parallels between AM and the internet revolution, citing the democratising impact the internet has had on the publishing and entertainment industries. Graham Tromans conjectured that, "the deskilling of design and production could be a big driver in its uptake". But other participants questioned whether AM would result in deskilling, at least at the higher end of the technology. This technology allows the creation of more complex objects, which would compel users to think back to first principles, asking 'what is it that this object is trying to do?' rather than 'how do I make this one better than the last one?' It may require more skill, not less"' (Royal Academy of Engineering 2013)7.

Thus, the greatest potential for AM remains in a context where customisation is paramount. For the medical field, it is important to acknowledge that some applications may be found in a more distributed setting, too, but their technical sophistication appears to remain limited for the time being. Current trends in the medical device industry also indicate a more application-based and specific centralised mode of production, as for example the fabrication of dental retainers (by InvisAlign) or of hearing aid shells (by various companies), two of the biggest biomedical sectors using AM.

Also in economic terms, the following major fields of medical biotechnology are believed to be strongly influenced and advanced through AM in the future:

- Surgical diagnostics and educational tools

- Prostheses and orthoses

- Drug delivery

- Organ printing / tissue engineering

- Food printing

4 For example: http://www.makepartsfast.com/additive-manufacturing-democratizing-design/

${ }^{5}$ https:/ / hbr.org/2015/06/the-limits-of-3d-printing

6 Wojciech Matusik, MIT Computer Science and Artificial Intelligence Laboratory, presentation at the 2015 MIT Europe conference, 14. October 2015, Vienna.

7 This may explain in part why at the odd print-shop, or even at pertaining events such as the Chaos Communication Congress, most 3D-printed do-it-yourself objects displayed still are remarkably simple in terms of both, function and geometry, and not to be compared with, for example, complex hacker software (Enno Park, personal communication). 
We will thus focus on these fields in the next sections, with personalised healthcare as an overarching aspect of several of these fields. Moreover, we will specifically look at the areas of dentistry and hearing aids, since they are already strongly shaped by the rise of AM)

\subsection{Techniques}

Formerly, AM was known as Rapid Prototyping. Its initial use was to quickly create simple objects meaningful for a particular related product. These objects supported the design process without being necessarily applicable. In recent years, however, AMT is entering industry as a way of manufacturing fully functional, end-user parts. It has grown from just being applicable for producing non-functional prototypes. AM is now about to revolutionise several fields in modern manufacturing.

When referring to $\mathrm{AM}$, the most common name used these days is $3 \mathrm{D}$ printing. However, according to the ASTM F2792-12a, it only refers to AM using printer technology such as print heads or nozzles.

To differentiate from competitors, AM systems manufacturers have created unique process names and material designations. Many of the different processes share the same technology and materials. The input to the system always consists of 3D model data and the fabrication occurs by joining materials in successive layers. The available materials, their unprocessed state and the mechanism by which these materials are joined, however, differ between the techniques.

Frequently, some confusion regarding the nomenclature jeopardises a clear understanding of the advantages and disadvantages of different techniques applied in various contexts. In the following therefore, we provide a brief overlook over AM techniques for fabricating parts from both liquid and solid phases.

\subsubsection{Fused deposition modelling}

In material extrusion based additive manufacturing, material contained in a reservoir is forced out through a nozzle by applying constant pressure. The diameter of the extruded material remains constant. The extrusion head moves in the horizontal plane predefining the shape of the layer. After one layer is completed, either the build platform or the head moves for starting the next layer, which adheres to the previous one (Gibson et al. 2015). Coming out of the nozzle, the material is in a semi-solid state. To ensure that the polymer remains in the predefined shape and adheres properly, quick solidification is necessary. Proper liquefaction can proceed via chemical changes ('curing', i.e. hardening agents, residual solvents, reactions with air, drying) or temperature, the latter being most

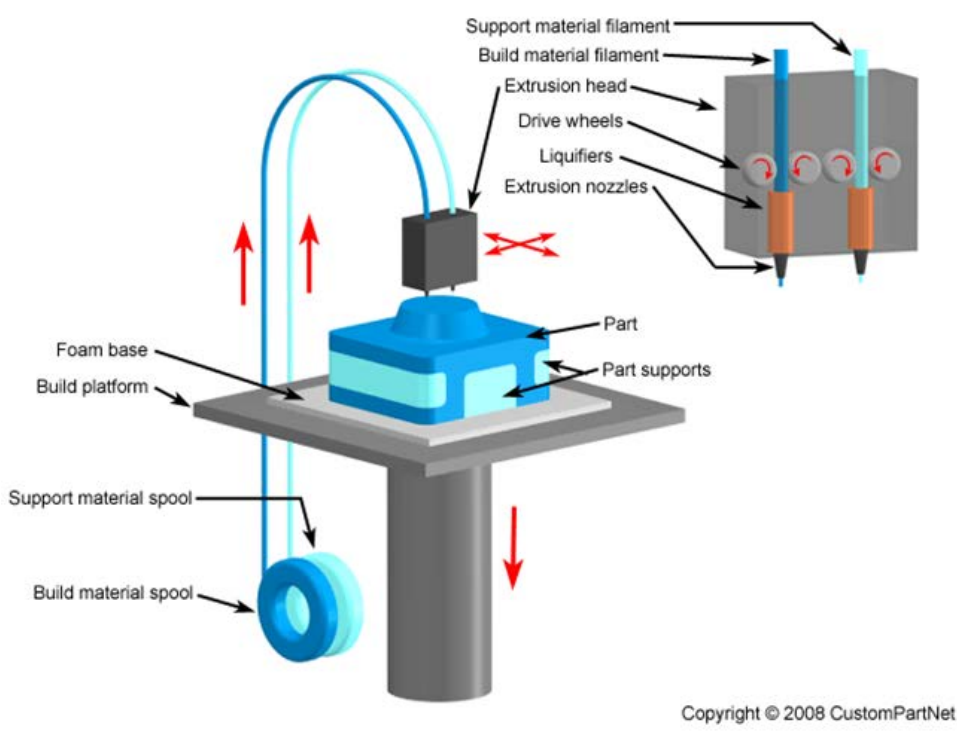

Figure 3 Fused deposition modelling. Source: CustomPartNet. http://www.custompartnet.com/wu/fused-deposition-modeling common.

The most common extrusion based AM is fused deposition modelling (FDM, Figure 3). Scott Crump introduced this process in 1989 (Crump 1992) and Stratasys, Inc. registered FDM as trademark in 1991.8

8 FDM - Reviews \& Brand Information - Stratasys, Inc. Eden Prairie, MN - Serial Number: 74133656, http:/ / www.trademarkia.com/fdm-74133656.html (accessed 9.2.15). 
Several other techniques, mostly derived from the open-source project RepRap (Jones et al. 2011), are based on a similar principle. Usually a pinch roller system pushes the filament material through a heated liquefier before it passes the nozzle. As support structures are required for bottom surfaces and overhanging features, modern machines use two or more filaments, one for part material and the other for supports that can be removed after the fabrication process (Gebhardt 2007). Generally, the filament can be any thermoplastic material coiled onto a spool. However, as viscosity control is crucial for the building process, crystalline polymers that have a distinct melting point, are difficult to process. Yet, the range of processible materials and the compact and low cost FDM systems, makes it one of the most widely used AMT (Gibson et al. 2015). It allows joining different raw materials in one part allowing for multi-colour (Pridoehl et al. 2014) and multi-material fabrication processes (Espalin et al. 2014). In addition, the obtained parts are among the closest to the bulk material properties among all AMT. The processible materials range from thermoplasts like acrylonitrile-butadiene-styrene (ABS) (Ziemian et al. 2012), polycarbonate (PC) (Domingo-Espin et al. 2014) to polyphenyl-sulfone (PPSF). In addition, biopolymers like poly-lactic acid (PLA) (Guo et al. 2015) and poly( $\varepsilon$-caprolactone) are processible (Teo et al. 2011). Including filler material or combining multiple filaments in the fabrication process, composite materials including ABS mixed with metals (Nikzad et al. 2011) or reinforced with carbon fibre (Ning et al. 2015) as well as PLA/TCP compounds (Drummer et al. 2012) are possible. The accuracy can reach up to $\pm 0.5 \mathrm{~mm}$ (Sood et al. 2010).

The biggest disadvantage of FDM is the long building time. The manufacturing of a solid part involves filling layers with closely packed polymer lines, where the line spacing and nozzle dictated cross-section determines resolution and density. This, in turn, determines the mechanical properties (Domingo-Espin et al. 2014; Sood et al. 2010). The print head has to move over large distances performing many changes in directions ${ }^{9}$. This building process makes FDM parts comparably rough and limited in accuracy. Depending on the process parameters and the AM machine used, parts can suffer from weak seam lines and delamination caused by temperature fluctuations during the process (Boschetto et al. 2013; Pérez 2002)

\subsubsection{Material jetting}

Inkjet printing heads move across a building platform jetting liquid material onto the previously formed layer (or the building platform itself), which is then solidified. The building platform moves down and the fabrication of the next layer starts (Figure 4). In this technology, droplet formation is the most critical parameter. Droplets must remain certain shape during flight and impact. The trajectory, velocity and size as well as the substrate interaction must be considered (de Gans et al. 2004; Gibson et al. 2010a). In most cases, the droplets are formed on-demand (drop-on-demand or DOD method), where the print head is physically positioned over the desired location on the substrate before ejection. A pressure pulse in the liquid drives a drop (typically 20-50 $\mu \mathrm{m}$ (Derby 2010) out of the nozzle. The liquid's density, the dynamic velocity and the surface tension determine proper droplet formation, which, in turn, poses stringent requirements to the processed material. Processible liquids have viscosity thresholds of 0.02-0.04 Pa s (Chua

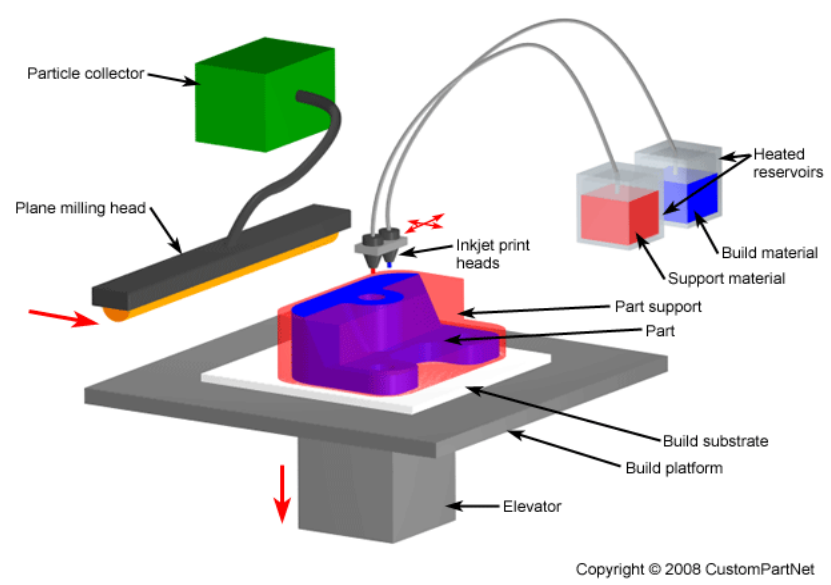

Figure 4 Material Jetting. Source: CustomPartNet. http://www.custompartnet.com/wu/ink-jet-printing

\footnotetext{
${ }^{9}$ Anisotropic material properties of fused deposition modeling ABS, http:/ / www.emeraldinsight.com.ezproxy.stanford.edu/doi/pdfplus/10.1108/13552540210441166 (accessed 8.31.15)
} 
et al. 2010). Upon the droplets impingement on the building platform or the previously fabricated layer, the material has to change from liquid to solid. The strategies range from cooling of melted materials (wax) (Ottnad et al. July 2012), evaporation of the liquid portion of a solution (Derby 2015) and curing of a photopolymer (Elliott et al. 2013) or other chemical reactions.

Depending on the curing process, the processible materials range from thermoplastics (molten droplets) and acrylic photpolymers to waxes.

\subsubsection{Vat photopolymerisation (stereolithography)}

A liquid photopolymer in a vat is selectively solidified by light activated polymerisation. Typically, a UV laser beam with small diameter passes an acousto-optic modulator (AOM) which turns it on and off (Figure a). The most common vat photopolymerisation technique is Stereolithography (SLA) (Figure 5), in which a scanner containing two rotating mirrors deflects the laser beam before it reaches the surface of a photopolymerisable formulation. The beam moves on the surface of the specimen according to the layer cross-sections curing the formulation to a depth slightly larger than the later movement of the Zstage ensuring cross-linking with unreacted functional groups of the previous layer. The coating system then delivers new liquid material and the illumination starts again. After fabrication, the operator removes the part from the building platform and cleans it from excess liquid formulation. This requires chemical developers or water (depending on the type of formulation). Afterwards, it is necessary to drain the finished part. In many cases, STL produces parts not fully polymerised. These "green part" are post-cured with UV light to improve their mechanical properties (Melchels et al. 2010).

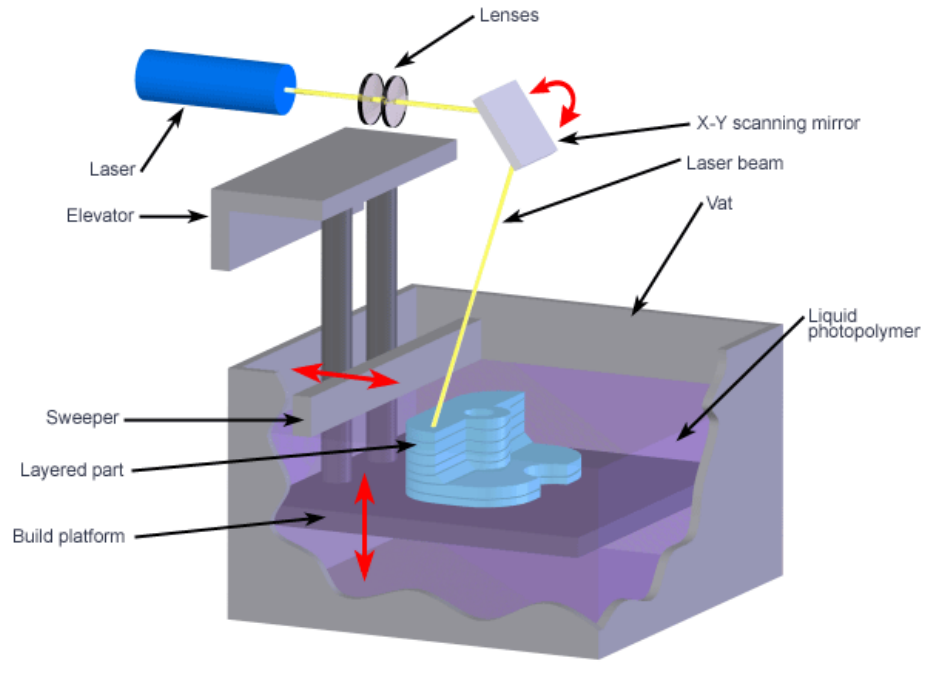

Copyright $\odot 2008$ CustomPartNet

Figure 5 Stereolithography. Source: CustomPartNet. http://www.custompartnet.com/wu/stereolithography

Chuck Hull introduced this technology in 1986 as the first AM (Hull 1986). Thus far, it is still the most precise one (Krini et al. 2015). Adjustment of focusing optics and photopolymerisable formulations can render resolutions below 100 $\mathrm{nm}$ (Torgersen et al. 2013). However, as with FDM, one layer cannot be produced in one illumination step. Despite the high scan speed of $0.2-0.5 \mathrm{~m} / \mathrm{s}$ (Stampfl et al. 2008), the fabrication time is still a bottleneck. With the rise of high resolution digital mirror devices and light sources with high illumination power, photomask lithography based AM emerged. Here, the machine fabricates the object top-down rather than bottom-up projecting light on a transparent, non-adhering plate from underneath. The building platform dips into the formulation from above. On one side, this eventually increases the mechanical forces during the separation from the bottom plate after the illumination. On the other, it ensures a smooth surface and prevents oxygen from reaching the polymerising surface. In addition, for low-viscous formulations, recoating is not required. In contrast to laser-scanning, the dynamic masks can cure the entire cross-section of the part at once. When using high power light sources, this reduces the process time significantly. Exploiting the full extent of the building platform, the process speed measure is vertical $\mathrm{mm} / \mathrm{h}$. A digital light projector (DLP) inside a video beamer projects the layer information on the photopolymer's surface defining the illuminated area. Depending on the focusing objective and the amount of micro-mirrors, the resolution can be up to $40 \mu \mathrm{m}$ laterally and $15 \mu \mathrm{m}$ in $\mathrm{Z}$ (Felzmann et al. 2012). 
For any illumination strategy, the range of materials is limited to photopolymerisable formulation only, which typically consists of reactive diluents (mono- and multifunctional groups), a cross-linker (high molecular weight backbone with several reactive groups) and a photoinitiator (creates polymerisation initiating radicals upon light activation) and, depending on the application, additional components, all influencing its reactivity, viscosity, reaction mechanism and the properties of the resulting polymer (Stampfl et al. 2008). Though certain properties can be adjusted with the cross-linker, the materials are limited to reactive components such as (meth)acrylates and epoxies, which often suffer from brittleness and toxicity preventing their use as functional parts (Dorfinger et al. 2015).

\subsubsection{Binder jetting}

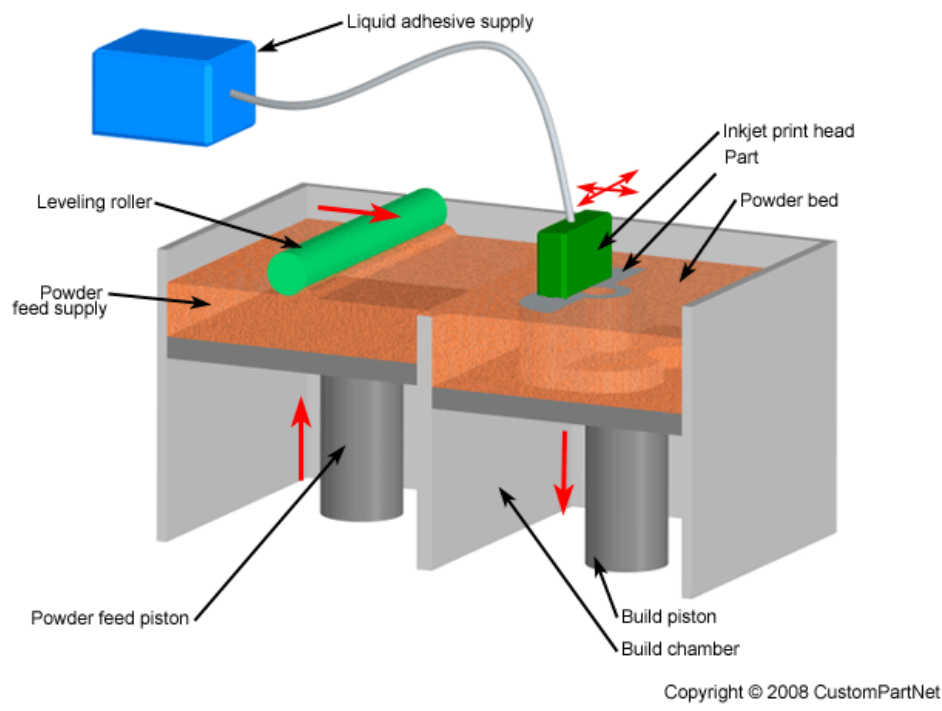

Figure 6 Three-dimensional printing. Source: CustomPartNet. http://www.custompartnet.com/wu/3d-printing
Binder jetting is a powder bed additive manufacturing technique that joins material powder using a liquid binder. The building platform positions a powder bed containing fine granulates of material. The distance to the powder bed's surface is adjusted to one layer thickness. The binder jet moves over the surface selectively joining material to a layer. The platform moves down and a blade supplies fresh material covering the previously fabricated layer. In this manner, the fabrication continues similarly as in material jetting. The original patent for binder jetting goes back to a Massachusetts Institute of Technology patent from 1993 that

was later licensed by Z-corporation (Sachs et al. 1993) under the name three dimensional printing (Figure 6). With print speeds of 2-4 layers per minute and comparably inexpensive powders and binders, this technology became important for conceptual models and prototypes with limited functionality. The accuracy, surface finish and part strength are lower than in other AMT, however postprocessing can eliminate the influences of the binder material. To adjust the binder's viscosity and fabricate parts in colour, various filler materials, additives and dies can be added. Support structures are not as critical as in fused deposition modelling, however, the design must account for removal of the residual powder. Various polymers such as ABS, polyamide, polycarbonate and polymethyl methacrylate are processible in addition to various photopolymers, ceramic composites, sand, aluminium and steel.

\subsubsection{Sheet lamination}

The raw material is a thin sheet of material usually coiled on two spools. Unwinding the sheet from the material supply and winding it on the used material spool, the sheets are placed on the building platform, where they are bond to the building platform (or the previously fabricated layer) and then cut to defined shape (bond-and-cut) or first cut and then bond (cut-and-bond). After this procedure, the building platform lowers, the spool unwinds an unprocessed portion of the sheet and places it on the previous fabricated layer. In the bond-and-cut method, the unused material is cut into small pieces in the serves as support. Their removal is however complicated, especially for small features. Cut-andbond facilitates easier material removal but requires external supports and layer alignment to attach the cut piece correctly. 
Lasers or mechanical cutters are used for creating the layer outlines. Depending on the material, gluing, adhesive binding, thermal bonding, clamping or ultrasonic welding joins the sheets together. The sheet thickness determines the accuracy in the $\mathrm{Z}$ direction and can be below $0.1 \mathrm{~mm}$ (Gibson et al. 2010b).

Based on sheet lamination, Laminated Object Manufacturing (LOM) (Figure 7) was patented in 1991 (Berman 1991) and soon commercialised by Helisys Inc. as one of the earliest AMT. Originally paper sheets were

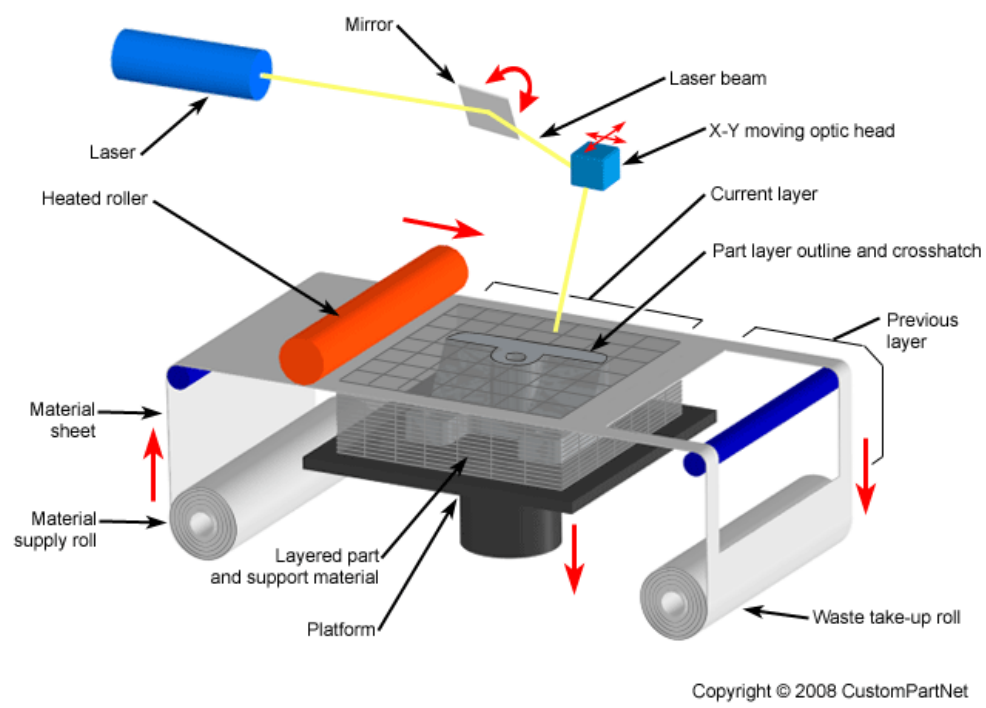

Figure 7 Layer object manufacturing. Source: CustomPartNet. http://www.custompartnet.com/wu/laminated-object-manufacturing joined together and cut with a $\mathrm{CO}_{2}$ laser.

Sheet lamination is an easy controllable and comparably cheap process. The parts have little shrinkage, residual stresses and distortion problems within the process. The raw material is of low cost and nontoxic. Machine and process costs are low and the excess material can be easily recycled. However, the layer bonding method also implies disadvantages. Often the raw material has to be coated to prevent moisture absorption and excessive wear. Depending solely on the flatness of the raw sheets and its deformation during cutting, high accuracy in the Z-direction is hard to achieve. The bonding between the sheets leads to inhomogeneous material properties. This has prevented sheet lamination from its use in fabricating functional prototypes. Rather, this technique is often used for sand casting and 3D topographical maps.

Although several polymers including polyvinyl chloride (PVC) (Solidimension Inc.) and composites can be fabricated, sheet lamination is popular for the fabrication of ceramic and metallic parts. Yet, up till now, the most popular lamination built material is paper with one sided thermoplastic coating.

\subsubsection{Powder bed fusion}

The underlying principle of powder bed fusion can be seen as a combination of binder jetting and SLA. As in the former case, a delivery system covers the building platform (or the pre-fabricated layer) with a thin and smooth layer of material powder. A focused energy beam (either light or electrons) is then scanned across its surface selectively joining material according to a predefined shape. As with any other AM, the building platform then lowers and the fabrication of the next layer starts. The residual powder remains and serves as support. Post processing involves part cooling and cleaning from residual powder. Further thermal processing and/or finishing steps are sometimes necessary depending on the material and quality requirements. In contrast to Material Jetting and SLA, thermal energy fuses the powder merging it to a workpiece.

Developed at the University of Texas at Austin, Carl Deckard patented Selective Laser Sintering (SLS) (Figure 8) in 1986 (Deckard). The company Nova Automation (later DTM Corporation) selling SLS machines was founded in 1988. Companies supplying machines and materials for selective laser sintering and the comparative process selective laser melting (SLM) have rapidly increased. To date, the biggest players are EOS GmbH, MTT Technologies, Concept Laser GmbH and Phenix Systems. 
Powder bed fusion allows processing almost any material in powder form that tends to fuse when heated (Kruth et al. 2003). The versatile fusion principle allows overcoming certain material property limitations arising from binders or required chemical functional groups existent in other AMT and allows creating engineering grade parts. However, the inhomogeneous thermal expansion and contractions during the process and during post-process cooling have to be tackled. A wide range of experimental and theoretical investigations are available (Bourell et al. 2014; Hussein et al. 2013; Song et al. 2011; Zeng et al. 2014) and intensive industrial as well as scientific research is ongoing optimising process conditions and materials.

One can distinguish four different fusing mechanisms (Kruth et al. 2005). In solid state sintering (1) fusion occurs without melting through diffusion; in chemical induced sintering (2), thermally activated chemical reactions bind particles; in liquid phase sintering/partial melting (3) a certain fraction of the powder melt, while other portions of the bed remain solid and (4) in full melting the particles are entirely melted to their liquid state. Most materials are amenable for solid state fusion, where the process temperature can be as low as $1 / 2$ of the material's melting temperature. This way, one can avoid large temperature

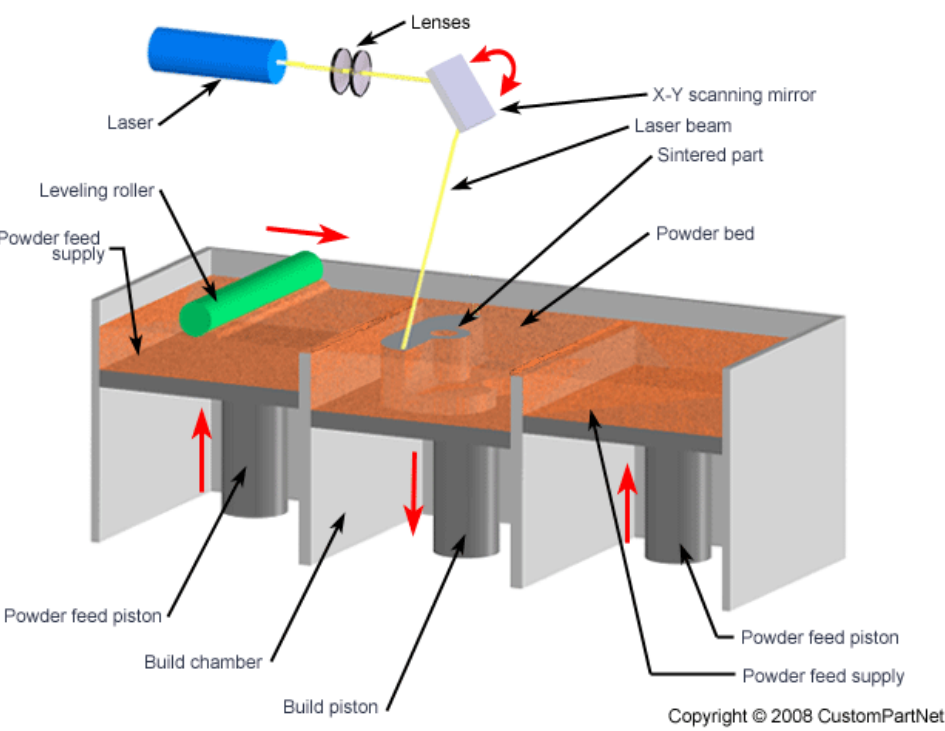

Figure 8 Selective Laser Sintering. Source: CustomPartNet. http://www.custompartnet.com/wu/selective-laser-sintering fluctuations and vast energy requirements. However, solid state fusion is slow and usually requires post-processing (Kruth et al. 2005). Full melting, in contrast, produces fully dense parts within a comparably faster process. However, only metals, metal-alloys and polymers can be processed. Inducing reactions to the surrounding gas, chemical sintering is used for creating ceramics. Liquid phase sintering/partial melting combines structural material in the solid and binder material in the liquid phase. Combining the characteristics of both full melting and solid-state sintering, composites and parts with superior properties can be created (Goodridge et al. 2012). A wide range of binder-structural material combinations are applied creating higher packing, allowing low temperature processing and avoiding dimensional distortions.

\subsection{Application Areas}

After this brief review over the most common AM techniques we now will have a look at a variety of AM applications within the health sector. The technological developments within the fields mentioned in the introduction are very varied and progress has been made to different degrees. Nevertheless, there are common features in some of these fields, for example, when it comes to the dependence on medical imaging.

In the following, we will provide a short overview over the state of the art, going through some of the most prominent fields of application. We will explain, respectively, main achievements as well as difficulties and challenges and also mention potential future developments. Using the example of surgical and diagnostic tools, we will deal with a number of issues that also apply to other fields in described in section 2. Therefore, the following sub-section is more detailed than the other parts of section 2 . 
In a second part, we will have a brief look at some developments that could be significant for human enhancement. However, to date there is no specialised methodology as eventual human enhancement applications would build on established AM methods used in other fields.

\subsubsection{Surgical and diagnostic tools}

One of the most important fields where AM has made a significant impact is the design and the fabrication of surgical and diagnostic tools. Departing from exact images of the area where a surgical procedure, for example, is to be carried out, tools to plan, to try and to guide these procedures are now available. Of course, this would not have been possible without huge progress in medical imaging using a variety of advanced technologies. Imaging is therefore an essential step and a prerequisite for any AM supported design and fabrication procedure.

Medical imaging has evolved considerably in the past few decades. Computer tomography (CT) and MRI has become indispensable in modern medical treatments. 3D visualisation, multiplanar reformation and image navigation made radiology pivotal for many clinical disciplines (Rengier et al. 2010). However, the use of screens limits the visualisation of 3D imaging data. With AM, one can overcome this limitation transforming 3D visualisations to physical objects. These objects resemble anatomical shapes and can serve a wide range of purposes. Regarding tools that can be fabricated range over a wide spectrum. Looking at products that do not remain in the body after the surgical procedure, we can classify fabrics in two major categories, anatomical models and biological structures for surgical planning and testing, and surgical tools and tools to guide surgeons during an operation (Giannatsis \& Dedoussis 2007).

The first group of fabrics are tangible models of the patient's specific anatomy (Ventola 2014) and fulfil a tailored visualisation and haptic purpose. They ease communication between stakeholders, i.e. surgeons, medical personnel and patients and facilitate well-conceived choices for the actual surgery. These models simplify education, conveying information and counselling keeping all involved parties informed on the procedures specifics throughout the treatment. Furthermore, AM models facilitate surgeon-training allowing the simulation of treatments without patient interaction. This is especially useful for the pre-simulation and rehearsal of complex medical cases. In neurosurgery, for example, such a patient specific pre-simulation is essential, as a narrow surgical corridor has to be followed and errors can have devastating consequences (Klein et al. 2013). These trainings are preferable to those on cadavers due to their limited availability, high costs and lack of resemblance to the actual patient (Banks 2013).

Surgical tools, on the contrary, are used during the procedure itself. Usually, a digital three-dimensional reconstruction of the patient specific site of interest is generated. A replicate of the surface is made which can be used in different ways during the surgery. For example, (Lu et al. 2009a; Lu et al. 2009b) fabricated drill templates for pedicle screw placement in spinal surgeries applying their tools in an surgical treatment. They claim that whereas a manual placement of the screws causes a high risk of bone weakening or lesion of the spinal cord, the AM templates fit on the unique morphology of the individual vertebra. The designed holes guide drills through a pre-planned trajectory, significantly reducing the operation time and radiation exposure for the members of the surgical team, as no images have to be taken during the operation. Lethaus et al. (2012) used anatomical surface reconstructions to pre-bend plates before the surgery that later were inserted after jawbone resection. The plates served as template for attaching bony grafts. The plates saved the time usually required for intraoperatively shaping moving this task to the preoperative planning stage and significantly simplifying the procedure for the surgeons.

The overall goal of both, anatomical models and surgical tools, is to help in decision making, in shortening the procedure, in improving the outcome and in ultimately improving patients' health (Morineau et al. 2009; Martelli et al. 2016). On the contrary to their benefits and potential, the underlying 
process of obtaining these models and tools requires a complex workflow involving mainly the following 3 steps: image acquisition, image processing and manufacturing.

Step 1 does not differ from conventional treatments in hospital nowadays, it does not necessarily involve AM considerations and established processes do not have to be changed. The medical data may derive from computer tomography (CT), magnetic resonance (MRI), positron emission spectroscopy (PET), 3D ultrasound or confocal microscopy. For creating 3D models for subsequent AM, CT and MRI are best suited to deliver medical images with appropriate resolution $(<250 \mu \mathrm{m}$ according to Siemens Healthineers ${ }^{10}$ depending on the type of structure under investigation. Due to advantages in contrast and easier conversion into 3D models, CT images are conventionally used for post-processing and AM fabrication. The data consists of a series of 2D images representing the model in stacked slices. The format can be either conventional (jpeg, bmp, tiff or x-ray raw image data) or represented in the DICOM format (Digital Imaging and Communications in Medicine, (Srivatsan and Sudarshan, 2015). The latter has developed as standard for storing, printing and transmitting information in medical imaging. The National Electrical Manufacturers Association (NEMA) holds the copyright (BS EN ISO 12052:20011).

Image processing constitutes the largest hurdle for AMs continuing introduction into the medical sector. The preparation of the data requires algorithms for the adjustment of the area contrast, for thresholding and segmentation as well as highlighting different areas of interest. At first, the procedure for creating a 3D data file for manufacturing does not differ from image preparation for visualisation. Tools for this task already exist on the market and are routinely used for planning and executing surgeries. ${ }^{11}$ However, whereas experienced medical personnel can still derive information from 2D stacked images with low contrast information, the reproducible and automatic conversion of stacked 2D medical data into a meaningful and anatomically correct $3 \mathrm{D}$ triangle mesh ${ }^{12}$ for $\mathrm{AM}$ fabrication poses stringent requirements to both operator and software. The tasks include the assignment of volumetric data, area enhancement, rendering of tissue surfaces and volumes as well as the object's internal structure (Srivatsan \& Sudarshan 2015). The conversion can be especially difficult when converting low contrast images derived from e.g. soft tissue. The workflow consists of the steps 1) creation of an image dataset, 2) thresholding, 3) region growing and 4) reconstruction of the model's internal geometry. Especially steps 3 and 4 require operators to possess both, deep computer and anatomical expertise making a strong cooperation of multidisciplinary teams indispensable. Despite the rapid development of respective software platforms, like Mimics from Materialise ${ }^{13}$, Anatomics RX and Biobuild, surgeons still perceive the time and costs spent to create a 3D printable model as the biggest disadvantage of AM in surgery (Martelli et al. 2016).

Having created a data file of a biomodel, the manufacturing can now proceed automatically. The build time depends on the type of AM process, the biomodel's size and its complexity. It usually requires a few hours. The most used processes for surgical tools are SLA, SLS, FDM, Powder Binding and Inkjet

10 Siemens Healthineers, SOMATOM Force, https://www.healthcare.siemens.com/computedtomography/dual-source-ct/somatom-force/technical-specifications (accessed 11.1.16).

11 Examples for DICOM data conversion into 3D models for additive manufacturing are Mimics http://www.materialise.com/en/medical/software/mimics, 3D slicer https://www.slicer.org/ , Anatomics RX http://www.anatomics.com/anatomicsrx/ordering-software/, 3D Doctor http://www.ablesw.com/3d-doctor/, Amira\& Avizo https://www.fei.com/software/amira-avizo/, Analyze http://analyzedirect.com/ and Slice-OMatic http://www.tomovision.com/products/sliceomatic.html

12 The STL file format is a data interface approximating a 3D object's surface via triangles. Three endpoints and the associated surface normal describe each triangle. The smaller the triangles, the more the model matches to its intended design. Smaller triangles, in turn, increase the file size and complicate computer processing. The STL is the common standard in AM and in various other computer aided manufacturing applications and is saved binary code or in ASCII (American Standard Code for Information Interchange)

13 http:/ / biomedical.materialise.com/mimics (accessed 11.2.16). 
printing (Rengier et al., 2010). The geometric complexity and accuracy obtainable by the powder bed technique renders this technology especially useful for surgical tools and guides (Salmi et al. 2013).

An assessment of feasibility of AM technologies in surgery requires a detailed analysis of the advantages and disadvantages surgeons experience with the use of this technology. Martelli and his group conducted a systematic review on 158 articles on AM in surgery performed in hospitals and published between 2005 and 2015 (Martelli et al., 2016). The main use of AM was in maxillofacial surgery and orthopaedic applications, where the contrast of medical images is high.

Accuracy is the major benefit that creates opportunities for AM in surgery. Accurate guides and templates for carrying out surgical procedures improve the positioning and therefore the quality and precision of cuts enabling highly symmetric reconstructions (Cohen et al., 2009; Feng et al., 2011). Reports overall highlight the possibility to create accurate guides and implant shapes. Correction and manipulation of the model can be omitted. Apart from the possibility of improved aesthetics, surgeons are also able to decrease patients' discomfort after treatment. Due to the low resolution of medical images, however, one cannot utilise the full resolution and shape capabilities of AM, which medical personnel perceive as one of the major drawbacks. Again, this becomes especially problematic processing low contrast images of soft tissue (Ngan et al. 2006).

The second most important opportunity of AM aided surgery is the time saved during operation. The biomodels act as tool for decision-making, as template for bending implants and/or as guides for cuts. This can make surgeons more effective during the actual medical procedure. The time saved during the operation reduces the accompanying risks and complications. These include blood loss, infections, anaesthesia time and postoperative complications. In addition, exposure to radiology during surgery can be minimised because continuous x-ray control is no longer necessary (Abdel-Moniem Barakat et al. 2014). This way, AM use in surgery may also decrease the use of analgesics and antibiotics (Martelli et al. 2016). Furthermore, a shorter procedure is less tiring for the surgeon and consequently reduces the chances for human errors.

For bony reconstructions in particular, the visualisation and pre-assessment of anatomical anomalies with help of AM has proven most successful (Ciocca et al. 2011; Leiggener et al. 2009). For example, (Hanasono \& Skoracki 2013) from the Andersen Cancer Centre in Texas compared treatments of 38 patients that underwent AM aided jawbone reconstructions between 2005 and 2011. The medical personnel bent titanium hardware on AM fabricated shapes. The bent implants were then used as bone flap fixations. The authors compared the operating time against a defect matched control group treated without AM surgical tools. They reported a decrease in the mean surgery time from 10.5 to 9 hours while increasing the placement accuracy of implants when using AM shapes.

Generalising the time saving with AM is difficult as it is highly case specific. Another study dealing with a similar task; the pre-bending of microvascular bony structures for implantation, reported a time saving of roughly 20-25 minutes without specifying the total operating time (Lethaus et al. 2012). In contrast to the time spent in the operation room, the preparation of an AM biomodel requires time for data processing and printing, which has been stated as a drawback. This time delays do not permit a respective medical procedure in emergency situations (Hammer et al. 2010). Estimations for the entire process from image acquisition to receiving the biomodel range from a working day to a couple of weeks, depending on the structure being fabricated in house or externally (Jacobs et al. 2008; Kono et al. 2013).

Again, the time spent in data preparation is stated most significant and labour-intensive. For example, (Jacobs et al., 2008) created anatomical correct 3D models for patients with complex heart diseases. They used most of the time and labour (3-8h) for the segmentation of the regions of interest and for creating AM data files. The authors particularly stress the need for manual segmentation to produce good models. Knowledgeable medical personnel has to participate in the data preparation process to provide the necessary anatomical interpretation and input (Kataoka et al. 2013). 
AM-aided education is regarded as the third biggest opportunity. Medical personnel perceive the biomodels as excellent tools for multidisciplinary communication and coordination (Wurm et al. 2011). It is possible to improve surgical decision making and reduce trial and error attempts, e.g. during fibular harvest and shaping (Hanasono and Skoracki, 2013). Specialists participating in a particular medical treatment can illustratively demonstrate their anticipated treatments helping in the discussion and identification of appropriate procedures. In parallel, the models serve as excellent tool for patient counselling and education. Patients can easier understand their treatment and knowing and understanding what will happen in the operating room. In addition, biomodels are useful as surgical training tool and for developing new procedures. It is possible to quickly acquire skills practicing on AM fabricated models with rare pathologic characteristics helping surgeons to acquire praxis and stay prepared for emergency situations (Yoo et al. 2016).

In contrast, the entire multidisciplinary process from acquiring images all the way to using printed models in medical procedure has been described as too complex and as requiring too many stakeholders. The organisational impact of AMs introduction in a hospital is high. A conventional medical procedure is already complex. The introduction of $3 \mathrm{D}$ printing software and the implementation of respective knowledge in a hospital surrounding is a big hurdle (Morineau et al. 2009). In a hospital setting, the surgeons play a huge and critical role at the preoperative planning stage coping with potential conflicts between the intent of the surgery and its potential impact on the patients' body functions. 3D visualisation and AM are knowledge based control systems allowing to predict and monitor the procedure. Whereas they may help to predict conflicts, lower the potential impact and better the outcome, their benefits are hard to grasp and foresee in particular cases. Surgeons often regulate conflicts through skill or rule based behaviour, with a lack of proper monitoring procedures (Wentink et al. 2003). The former stems from the trained surgeon's sensory-motor loop and the latter on rules constructed from previous experiences. In contrast, knowledge based behaviour involves a control based on conceptual models allowing an analysis of the environment and purposes to attain. In a recent report, the documentation of conflict occurrence in a surgical procedure only increased with increasing complexity of the surgery when knowledge based control mechanisms were in place (Morineau et al. 2009). Due to the lack of monitoring, it is therefore often hard to determine whether an AM aided procedure is beneficial or only distracting the surgeon.

Another major disadvantage for the use of AM in a hospital setting is the costs for CAD software and AM equipment and/or the costs of producing parts externally (Hanasono and Skoracki, 2013). AM pays out in the complex, non-standard procedures that are hard to standardise and to monetarily quantify. A cost benefit calculation is therefore delicate and dependent on the access to services, skills of medical image processing, surgery skills, risk-costs and many other factors. (Hanasono and Skoracki, 2013) estimated the costs of each AM fabricated models as ranging from $\$ 3.000-\$ 4.700$ ( $€ 2.750-4.300$ ), costs that might only be justified in special complex procedures, especially when considering that AM based services are not necessarily included in the medical coverage (Izatt et al. 2007). However, the costs vary between different reports. For creating patient-specific drill guides, (Schweizer et al. 2013) reported costs of only $\$ 220-\$ 230(€ 200-210)$ per piece.

Mechanical properties of standard AM parts do not necessarily mimic those of natural tissue; they are often reported to be too rigid and fragile to serve as proper anatomical models (Schievano et al. 2007). However, poor properties are often mentioned in conjunction with inkjet-printed parts. A wider material selection is available for other processes. When selecting the proper process, the parts' feasibility for sterilisation also have to be taken into account (Hung et al. 2008).

In summary, AM has become a very important tool in surgery already and will play an even bigger role in the future. However, their usual presentation as time saving tool is not necessarily correct. Biomodels in general can save operating time but fabrication of these models is time consuming. Furthermore, data preparation and creation of digital models requires skilled personnel and software. Reviewing the clinical reports, the latter issue seems to be the one with most organisational, time and cost impact. 
Further development in software, automation and training of personnel in hospitals will be required to ensure proper usage of AMs potential. Hospitals will eventually develop their own manufacturing centre that focuses on creating physical biomodels out of medical imaging data. These centres will eventually merge with the radiology department. Presumably, they will not rely on AM and other processes enabling the direct and fast conversion of CAD into complex physical objects.

In general, 3D imaging devices have the potential to enlarge the information given to the patients in relation to their situation after surgical interventions. Advantages go from a better understanding of the deformities/fractures, planning accurate correction of deformity to the reduction of surgical time, blood loss and use of anaesthesia. Important is also the potential to customise implants and instruments. 3DImaging devices are used also for educational purposes to enhance the imagination of the patient and her understanding of the entire procedure. While none of these applications are used for the purpose of human enhancement as defined in this report ("modifications aimed at improving individual human performance and brought about by science-based or technology-based interventions in the human body"), 3D imaging devices are used in the field of plastic surgery not only to support therapeutic purposes ${ }^{14}$ but also in the area of "cosmetic" surgery; and this area is one of the (very few) wellestablished areas of (non-therapeutic) human enhancement. Patients can, for example, receive a 98.5\% accurate, 3-dimensional image of what their modified body parts will look like after breast augmentation (Roostaeian \& Adams 2014).

\section{Key findings:}

- Anatomical models are reconstructions from a patient's site of interest. They ease communication between stakeholders, education and training and allow simulating the treatment without patient interaction.

- Surgical tools are templates reconstructed from a replicate of an anatomical surface. A replicate of this tool is made and used in different ways for easing surgery, e.g. as a template for the correct placement or for the bending of an implant.

- Medical images from CT and MRI form the basis for fabricating these items. The image contrast and the ability to convert these images into 3D printable data files determine the quality.

- Main uses are maxillofacial surgery and orthopaedic applications.

- The accuracy, time saved in surgery and educational opportunities are reported as biggest advantages.

- Major drawbacks are reported to be the process complexity involving multiple disciplines, the costs (CAD software, AM tool,...) and the mechanical properties of the parts.

- Surgeons perceive image processing and the creation of 3D printable data files to be the biggest hurdles for the introduction of AMs into the field.

\subsubsection{Digital dentistry and hearing aids}

Conventionally, dentists used to take an image from the treatment site and pour a plaster model with which they constructed a wax pattern. This pattern was then invested ${ }^{15}$ and replaced with a permanent material such as metal, silicone, acrylic or ceramic (Sadan et al., 2005).

In terms of workflow, dental procedures are different process than other surgeries. The oral cavity is much more accessible and the tissues under investigations are hard materials that are easier to fabricate and replace by prosthesis than any other part of the body. Both, during surgery and during later usage of the implant, they have the least side effects.

14 See https://3dprint.com/117889/3d-print-to-reconstruct-faces/.

15 This term refers to the pattern being invested (surrounded) with a refractory material. Another term for it is investment casting. 
Dental laboratories are separate from hospitals and are the early adopter of new technologies and materials. The automation of the production for dental restorations started 20 years ago and CAD/CAM systems were introduced as early as 1980 (Beuer et al., 2008; Strub et al., 2006). Since the rapid increase in computational power and data acquisition capabilities made the workflow for obtaining a restoration more and more digital. Products such as (Torabi et al., 2014):

- Fixed and removable partial dentures

- Wax patterns for the dental prosthesis

- Zirconia prosthesis and moulds for metal castings and

- Maxillofacial prosthesis

are nowadays routinely fabricated using in house or external CAD/CAM manufacturing services. For dentistry, the workflow of obtaining a physical object from an anatomical site of interest does not differ from the one described for surgical guides and tools. It consists of 1) digitalisation, 2) data processing and 3) manufacturing.

Data acquisition is essential to evaluate and record size and form of anatomical structures providing information on soft and hard tissues. Common techniques for this task are CT, cone beam CT, micro CT and MRI. In addition, scanners based on 3D laser scanning, structured light techniques, sterophotogrametry and 3D surface imaging are becoming popular for dentistry (Karatas and Toy, 2014). Independent from the type of imaging technique, the need for high speed, high density and resolution to gather small feature sizes has advanced the development of 3D imaging in recent years (Karatas and Toy, 2014). However, despite these advances, the common approach to scan a model from the patient's oral cavity still includes the fabrication of a cast prepared by conventional impression methods that are later digitised. However, the quality of direct imaging is improving. Some articles already report better performances with the direct method (Güth et al., 2013; Vecsei et al., 2016).

A variety of software tools are available to convert the digital model into restoration designs such as crowns, bridges and partial denture frameworks. As described in 2.2.1, data preparation can be difficult, requiring software skills and anatomical knowledge. However, from a software perspective, dental restoration is a narrower task than the fabrication of surgical aids. Software packages are advanced, user friendly and already integrate an entire database for storing, ordering and simulation. Examples are Soredex, ${ }^{16}$ Sirona, ${ }^{17}$ Dentca ${ }^{18}$ and iTero ${ }^{19}$. iTero, for example, is a combined imaging/data processing tool that allows to optically scan the patient's oral cavity, allows data manipulation such as the insertion of teeth or their alignment and allows data exchange with external vendors for the fabrication of appropriate restorations. Soredex provides tailored software for data gathering and preparation for the treatment of cysts and tumours, endodontic studies, maxillary sinus studies, for tracking nerve canals and many other applications. Dentca and Sirona cover the complete workflow, from imaging to the final part, giving dentists and patients a specific procedure to follow. All of the packages allow the digital insertion of restorations and the conversion of the file into printable/ machineable data files. The possibility of simulating and visualising the procedure allows for a decreased number of appointments as compared to conventional procedures, a decrease duration of the prosthesis manipulation, the opportunity of standardisation for clinical research on removable prostheses, an easy reproduction and manufacture of trial denture as well as better quality control (Bidra et al. 2013; Bilgin et al. 2016). Scanner devices together with digital technology made previously manual tasks easier, faster accurate and more predictable.

Manufacturing is not linked to AM only. In fact, most of the dental prosthesis and products are made by conventional subtractive manufacturing (milling, machining) due to the higher speed of fabrication,

\footnotetext{
16 http://www.soredex.com/en/digital-imaging-software/

17 http://www.sirona.com/en/products/digital-dentistry/

18 https://www.dentca.com/

19 http://www.itero.com/en
} 
the wider range of available materials, the established supply chains and the better surface quality of the obtained parts (Beuer et al. 2008; Vandenbroucke \& Kruth 2007). The most common materials processed are metals and ceramics, materials only available with certain, costly AM technologies. However, despite AMs niche existence to this date, the technology has high potential for dentistry and is becoming increasingly popular due to its potential to overcome the limitations of machining such as (Torabi et al. 2014):

- Material waste: machining produces a large amount of material waste and creates wear on tools (especially when machining hard and brittle materials such as dental ceramics). In a typical fabrication procedure of a dental restoration, approximately 90 percent of the raw material is removed in machining (Davis 2010).

- A precise fit of the restoration to the site of interest is governed by the smallest machining tool available and dependent on the type of fabrication process and the processible materials. Hollows and undercuts that maybe required to replicate the exact tooth geometry, cannot be fabricated with subtractive manufacturing

- Milling tools can wear out fast, especially when processing ceramic materials resulting in high maintenance costs and downtimes.

- Cracks can develop in ceramic surfaces induced by the load of the machining tools on the material

The fabrication of surgical guides and tools for jawbone reconstruction has been mentioned before as one of the most established applications for AM in biomedicine (Stoetzer et al. 2011). Similarly, AM is widely used in dental surgery for implant planning and placement purposes (Lal et al. 2006). This has proven especially useful for complex cases, for example the complete oral rehabilitation with implants (Bentz \& Balshi 2012).

AM offers the possibility for automatically creating wax models. These models can later be invested, moulded and then casted similarly to the conventional way. ${ }^{20}$ The creation of the wax model (wax-up) itself is thereby one of the most crucial and labour-intensive steps in the fabrication of the dental restauration and AM has the potential to automate it (Liu et al. 2006; Sun \& Zhang 2012). The digital model serves as basis for designing the wax pattern and low cost AM such as FDM or 3DP for its fabrication. Compared to a manual fabrication procedure, this approach is believed to result in high production rates, easy quality control and high precision wax patterns as well as the reduction of required finishing work on the casts (Azari \& Nikzad 2009; Liu et al. 2006). Several studies on the fabrication of moulds for the casting of restorations exist (Joo et al. 2016; Williams et al. 2006; Wu et al. 2001). However, despite the credited accuracy benefits, a few reports also criticise the large deviations of restorations obtained with AM wax-ups comparing fabricated models with those obtained from conventional machining and/or manual processes (Munoz et al. 2016).

Reports on the direct fabrication of restorations are rare. However, using SLS and SLM, successful clinical trials are already published (Tara et al. 2011). Among the applications, the fabrication of partial denture frameworks is the earliest direct fabrication procedure, with the first clinical use already in 2006 (Williams et al., 2006). AM techniques for zirconia ceramics are rapidly developing (Anssari Moin et al. 2016; Silva et al. 2011) showing great potential to digitise and automate the direct fabrication of ceramic implants.

For dental applications, part sizes are small enough inducing no restrictions on the build size (Feng et al., 2010). Several dissimilar parts can be fabricated at once, during one process, with minimal additional build time and without the necessity of retooling and/or reclamping. In addition, workpieces with different graded materials, such as graded porosity, graded composition and topology tuned mechanical properties can be fabricated providing a large freedom in dental restoration design (Traini et al., 2008). Designing porous constructs allows for tailored biocompatibility such as osseointegration (Gagg et al., 2013).

\footnotetext{
${ }^{20}$ http://www.infodentis.com/fixed-prosthodontics/wax-up-and-metal-casting.php
} 
The accuracy of AM is considered among the biggest advantages of AM in dentistry. Parts can be made to fit the patient's hard and soft tissues (Davis, 2010; Webb, 2000). Quante et al. found the accuracy of implants made from metal and metal alloys to be in the range between 67 and $99 \mu \mathrm{m}$ producing significantly finer features than possible with conventional machining (Örtorp et al., 2011; Quante et al., 2008).

The production procedure of successively adding layers induces an external surface with stepped and coarse morphology (Huang et al., 2010). This can have adverse effects on the parts' functionality as implants as well as mould. When directly fabricating implants the porosity of metal parts fabricated by SLS can reduce their durability (Traini et al., 2008), which may especially be critical during the fabrication of intraoral prosthesis that have to withstand considerable loads. Furthermore, the number of available materials suitable for the direct AM fabrication of restorations is still limited (van Noort, 2012). Due to the costs of metal and ceramic AM machines, the indirect fabrication of restorations via intermediate wax models is therefore still dominating the direct fabricating. The most common metal and ceramic processes (SLS or SLM) might still be out of the financial reach of many dental laboratories. However, apart from the costs of equipment, the time and competence to make a printable 3D model is still perceived as the biggest hurdle for AMs broader use in dentistry.

A way to work around this problem and still benefit from CAD/CAM manufacturing in general is outsourcing (Beuer et al., 2008). Many laboratories nowadays have scanners connected with a production centre; the recorded data is directly sent to the centralised entity via the internet. There, the parts are manufactured and sent back to the responsible laboratory. In this scenario, the laboratory's initial investment to benefit from CAD/CAM manufacturing is the scanner/ digitalisation device and respective software. The laboratory is still responsible for the design of the restoration and does not have to rely on one single manufacturing technology (such as SLS or a milling). In addition, the laboratory does not have to select the most suitable technique for fabricating their design. On the other hand, they have to rely on one manufacturer's/supplier's framework and will lose the income from producing the restoration.

The outsourcing strategy has led to the rapid application and acceptance of AM in digital dentistry. Companies like Stratasys already provide particular orthodontic AM setups producing tailored materials for temporary in-mouth placements and diagnostic wax-ups. ${ }^{21} \mathrm{~A}$ variety of commercial dentistry products relying on the outsourcing principle are now available. We get access to new, almost defect free industrially prefabricated and controlled materials. Dentists store patient data through a standardised chain of production constantly improving precision, planning and efficiency (Beuer et al., 2008). Several companies provide a complete framework for obtaining restorations. For example, the Sirona group provides all necessary equipment and production means to fabricate bridges and crowns. InvisAlign provides the entire process chain from scanners operating on site, simulation of tooth positioning, software to create tooth aligners, all the way to ordering networks, centralised fabrication and appropriate logistic channels. In addition, they also train and certify personnel and laboratories to work with their processes. The company continues to grow and is offering more than 17 million unique aligners every year (Wohlers et al., 2016).

Companies have created their own digital workflows and try to constrain users to only use their processes and related products. However, with increasing data exchange between components in dental CAD/CAM and continuous standardisation and accessibility, it is very likely that dentists of the future will work with different sources, software packages and manufacturing systems in an interconnected supply network. In this network, AM will play a role, but it will probably not revolutionise the field entirely (van Noort, 2012).

${ }^{21}$ http://www.stratasys.com/3d-printers/dental-series/objet30-orthodesk 
In a workshop on 'The 3D Printing Revolution and the Dental Sector: Opportunities and Challenges' held in European Parliament premises on 30 November 201622, there was, according to a participant, a consensus that the training of dentists, further research (for example on biocompatibility) and legal questions (for example reference standards) are key challenges. Some concerns were raised about potential job losses (for example with regard to dental lab technicians) but not discussed in in great detail.

As with the dental industry, the fabrication of hearing aids is a lucrative, highly competitive business (Figure 9). The hearing aid industry is an early adopter of modern manufacturing technologies. Here, the fit of the In-The-Ear hearing aid is more important than its mechanical properties.

AM for the production of hearing aid shells is an even greater success story as AM in dentistry. The fabrication of shells of in-the-ear hearing aids has almost entirely shifted to AM. ${ }^{23}$ These products need to integrate electronic signal processors, microphones, a battery and a loudspeaker posing significant constraints on power management and imposing acoustic problems (Lotz, 1998). The hearing aid has to fit tightly to avoid feedback resulting in squealing noises inside the ear. Similar to other related biomedical industries, the potential of AM strongly aligns with the possibility to create a digital image of the anatomical site without any manual steps such as such as sculpting and moulding.

The application of custom AM hearing aids is not under public attention, however, the hearing aid industry already shifted its operation to almost entirely producing shells with AM (Sandström, 2016). The market penetration is highest among all of its potential biomedical applications. Deloitte stated in a report from January 2015 that nearly 15 million hearing aid shells are made by AM worldwide. ${ }^{24}$ The industry transitioned to the technology already in the years 2008-2009. Six companies control $95 \%$ of the market, these are Siemens, Oticon, Phonak, GN Resound, Starkey and Widex (Sandström, 2016). Europe is the largest market followed by the US and the need is growing steadily. ${ }^{25}$ The gross margins are high and, unlike other electronic products, the retail price increases over time. ${ }^{26}$

${ }^{22}$ For a video covering he entire workshop, see https://www.youtube.com/watch?v=Nlf3oGE6ehE. For the workshop programme, see http://www.bec.it/download/aioprogramma.pdf.

${ }^{23}$ Hearing aids can be roughly classified into behind the ear models and in the ear models, the latter being the more comfortable to wear but also the more expensive and with the higher customisation requirements.

24 https://www2.deloitte.com/global/en/pages/technology-media-and-telecommunications/articles/tmtpred-3d-printing-is-revolution.html

25 http:/ / hearinghealthmatters.org/hearingnewswatch/2013/research-firm-analyzes-market-share-retailstores-prospects-of-major-hearing-aid-makers/

26 https:/ / www.audicus.com/hearing-aid-prices-a-comparison/ 
In the field of dentistry, the only application discussed in the field of human enhancement is the enhancement of the digital simulation of patient's teeth for cosmetic purposes. This application does not have directly to do with 3D printing but takes advantage of 3D printing in terms of precision.

Since with 3D scanning, CAD/ CAM technology, audiologists, manufacturers, service centres have the potential to design and produce the perfect customised hearing aid shells, ear moulds, noise protection and soft moulds, it can be in principle expected that devices will be created which permit to overcome the average human range of sound. However, at the moment there is no discussion about these applications, since the field is in the making and it is targeted to therapeutic goals.

\section{Key findings}

- Data acquisition and 3D model preparation are essential parts of the digital dentistry process.

- A large variety of software packages exists that allow visualisation, simulation of the procedure and conversion into printable data files.

- Today, conventional machining dominates the manufacturing of implants; AM has a niche existence but high potential.

- Usually, low cost AM models are used for creating wax patterns for subsequent moulding.

- The direct fabrication of replacement/implants is reported and has the potential to fully automate the entire process chain.

- The advantages of AM in the field are: low material waste, precise fit, lower maintenance and downtime costs and a lower probability of defects on the implant surface as well as more biocompatible designs.

- The bottlenecks for end use production are: low surface quality (stepped surfaces), limited number of available materials, internal porosity of ceramic parts and the high cost of AM machines.

- Many dental laboratories utilise the digital workflow to outsource the production.

- The fabrication of hearing aid shells is one of the most successful AM applications.

\subsubsection{Orthoses and prostheses}

Orthoses are devices to correct or enhance the use of a part of the body, whereas prostheses replace the body part in part or entirely. ${ }^{27}$ Providing support and comfort during the healing process, orthoses reduce shock, inflammation and swelling. Prostheses, in contrast are permanent, intended to allow patients to live/continue a usual life despite the absence of the specific body part. Both, orthoses and prostheses (O\&Ps) are intended to help people with congenital limitations and people who had injuries or suffer from the consequences (e.g. amputations) of certain conditions such as diabetes (Chitragari et al. 2014). O\&Ps can be functional, as for example artificial legs or exoskeletons (Jiménez-Fabián \& Verlinden 2012) and/or cosmetic as for example artificial eyes (Wavreille et al. 2013). By quantity and revenue, foot orthoses are the most common O\&Ps.

Both can be either acting external or be implanted (Ramu et al. 2015; Udoekwere et al. 2014). The fabrication of O\&Ps with additive manufacturing offers huge potential as the devices can be built as a whole, integrating joints and moving parts, which are more convenient to wear and more aesthetic. ${ }^{28} \mathrm{In}$ addition, AM offers the freedom to design internal structures and allows combining materials in one build. In this regard, AM allows to reduce weight, implement material property gradients (stiffness, elasticity, hardness, etc.) and integrate functioning parts like sensors and actuators. The design freedom even allows to fabricate biomimetic structures such as grasping devices leveraging surface effects (Arzt et al. 2003; Autumn 2006). AM is, for example, used to replicate a physical model from soft tissue data that is mirrored from the intact hand. With this mould, parts of a hand prostheses or orthosis can be

\footnotetext{
27 https://www.humantechpando.com/whats-the-difference-between-orthotics-and-prosthetics/

28 http:/ / www.3ders.org/articles/20150623-german-giant-ottobock-teams-with-unyq-to-develop-stylish-3dprinted-prosthetics.html
} 
made. This could reduce the number of steps needed for custom fabrication and improve the fit of the end product (Jin et al. 2015). The design freedom also allows to improve aesthetics and monitoring and continuous product development (e.g. to correct an abnormal posture). With respect to conventional subtractive manufacturing processes, AM can aid to reduce material waste, potentially offering a cost advantage.

In contrast to surgical aids, this subsection will focus on devices that have direct purpose in aiding the patient outside of the hospital. Dental restorations, described in 2.2.2, essentially are also O\&Ps. Here, we nevertheless want to draw a distinction, as, regarding available scientific literature, the fabrication of dental restorations with regards to AM seems far more advanced than that of fabricating other O\&Ps. Also, we want to distinguish O\&Ps from other implants that deliver medication (drug delivery devices) and those that provide support for creating tissues and organs (tissue engineering constructs); these will be the topic of the following sub-sections.

One important aspect of the use of AM for the fabrication of O\&Ps is the aim to fit the O\&Ps to the anatomic sites of interest. Several clinical studies on O\&Ps have shown that this is the most decisive factor for functionality and customer satisfaction (Jin et al. 2015). A survey of 581 veterans and service members wo lost limbs in the Vietnam or Iraq wars revealed the socket fit as being one of the major issues (Berke et al. 2010). Insufficient fit can lead to the alteration of the patient's posture. For example, a person with a amputated limb may favour and stress the intact leg more than the one with the prosthesis leading to back pain and degenerative changes such as osteoporosis (Gailey et al. 2008). Here, AM can provide tailor fit O\&Ps that may potentially improve the outcome. Also, as described earlier in the sub-section on surgical aids, the traditional fabrication of O\&Ps via the plaster moulding or vacuum forming technique is labour intensive and requiring several casting, fitting and moulding steps. The process can be eventually simplified with the use of AM.

The process of fabricating an AM orthosis or prosthesis, proceeds according to the steps (1) imaging, (2) data processing/ geometry modification and (3) fabrication (Jin et al. 2015). Usually the device is made as mirrored replica of the healthy body part (limb, hand, part of the skull, etc.) The imaging data is often obtained from a direct scan or indirect (negative mould from e.g. a foam box) 3D laser scan of the healthy limb (Jin et al. 2015; Marchelli et al. 2010; Telfer et al. 2014). To design a custom O\&P, the shape of the device is fitted to the surface profile of a body scan (e.g. 3D laser scan). This can be done with e.g. Tracer $\mathrm{CAD}^{29}$ or CanFit, ${ }^{30}$ two of the many, rapidly developing CAD/CAM solutions tailored for the creation of custom O\&Ps. Many of those systems already allow exporting designs in an AM file format. In many reports, however, the authors used several software solutions (e.g. Magics, SolidWorks, ${ }^{31}$...) to obtain a manufacturable design (Jin et al. 2015). Telfer et al. point out the skills and experience required to obtain the final product and the limited reproducibility of the outcome that is mainly dependent on the operator (Telfer et al. 2014; Telfer et al. 2012a)(Telfer et al. 2014; Telfer et al. 2012a). For the fabrication of custom O\&Ps mainly SLS and FDM proved successful.

The AM of foot orthoses is quite widespread. Milusheva et al. published the procedure of producing personalised foot orthoses using laser scanning and AM as early as 2007 (Milusheva et al. 2007). The cost effectiveness of the AM approach has also been demonstrated (Dombroski et al. 2014). Several unique designs were made possible through AM, including tailored reduction of the load on the metatarsal heads, ${ }^{32}$ adjustable stiffness and the integration of sensors (Pallari et al. 2010; Telfer et al. 2014; Telfer et al. 2012b). Combining data acquisition with laser scanning and AM, SOLS33 commercialised the fabrication of custom insoles in 2013. Products are directly available to end users

\footnotetext{
29 https://www.willowwoodco.com/products-services/omega/

$30 \mathrm{http}: / /$ vorum.com/cad-cam-prosthetic-orthotic/canfit-design-software/

31 http://www.solidworks.com/

32 Five long bones in the foot located between the bones of the hind- and mid-foot and the toes.

33 http://www.sols.com/
} 
and can be designed with simple photographs as input and a mobile app ${ }^{34}$. Another example of the application of AM is the treatment of children's clubfoot ${ }^{35}$ where conventional treatment involves a series of correction steps. In an analogue to the InvisAlign approach (see the sub-section on digital dentistry), this treatment requires several casting steps and custom orthoses to allow patient the development of a correct foot posture (Vijayaragavan et al. 2014). This has market potential for AM.

AM fabricated ankle foot orthoses are similarly widespread. Faustini et al. (2008), for example, compared the performance of conventional carbon-fibre orthoses to Nylon 12 devices made by SLS. The AM design showed better damping as achieving adequate stiffness as well as withstanding a whole range of destructive tests. The authors point out the automated procedure and geometric complexity obtainable. In this regard, it allows for the reduction of labour-intensive process steps together with a refinement of the devices' passive dynamic response. The iteration of ankle-feet orthoses design with regards to performance is possible through finite element modelling ${ }^{36}$ and topology optimisation ${ }^{37}$ (Pallari et al., 2010). This way, the design freedom of AM allows for increased opportunities to tailor device performance. In several gait parameter study, AM fabricated ankle-foot orthoses allowed patients similar support as their conventional fabricated counterparts (Creylman et al. 2013; Harper et al. 2014). As the fabrication procedure involves fewer steps, it has credited potential for studying effects of design changes on gait performance (Jin et al., 2015).

AM was also used for creating prosthetic socket fits for amputees. The shape of the residual limp has to be carefully considered to circumvent points of high contact pressure. This is especially challenging as the volume of the residual limb may change over time, especially during the time immediately after the amputation. Socket replacements may frequently be necessary. Here, again, the combination of digital imaging and AM has potential. Apart from customisation, mechanical labour reduction and rapid supply of tailored prosthesis, AM allows for benefits such as sockets with different mechanical properties in different socket regions to replicate the load conditions in a healthy limb. These prospects were recognised very early, straight after the development of AM and digital imaging techniques. The residual limp and the fabrication of an AM prosthetic socket is one of the pioneering applications. The 3D laser scanning and the fabrication of a prosthetic with SLA and SLS were already reported in the 1990s, immediately after the AM and digitising technologies had become available (Marcus et al. 1991; Zupko 1992; Rovick et al. 1996). An amputee already wore an AM socket in 1992 following many clinical studies (Rogers et al. 2009) and customised designs for compliance.

However, sophisticated designs were discarded due to the high costs and complicated design process involving many software packages that directed research back to single wall designs (Bourell et al. 2001). Low cost AM processes were employed and AM equipment suppliers for socket fabrication such as Rapid Manufacturing Machine (Ng et al. 2002) and Squirt-Shape emerged ${ }^{38}$. In a case, a patient wore

${ }^{34} \mathrm{http}: / /$ lermagazine.com/issues/december/sols-harnesses-additive-manufacturing-data-capturetechnologies-to-innovate-custom-insole-space

35 Deformity at birth, where the affected foot appears to have rotated internally at an ankle. Without treatment, people tend to walk on their ankles or sides of their feet. With treatment, the vast majority of patients recover during early childhood (Miedzybrodzka, 2003).

36 Finite element modelling describes an entire problem as system of small parts (finite elements) described by simple functions. It is a numerical technique linked to computer aided design providing approximate solutions to boundary value problems. In engineering, it is used to calculate and visualise distribution of stresses and displacements depending on external influences such as thermal, electromagnetic, fluid and structural working environments (Reddy 1993).

37 Topology Optimisation is a computational method minimising material usage while optimising its layout depending on certain boundary conditions. It relies on repetitive use of finite element modelling to perform compliance calculations. Using topology optimisation, engineers find concept designs that fits best to set design constraints (Bendsøe \& Sigmund 2004).

38 http:/ / www.engineering.com/DesignerEdge/DesignerEdgeArticles/ArticleID/10255/The-Squirt-Shape3D-Printer--a-Moonshot-Project.aspx 
a prosthesis fabricated by the latter for as long as 34 months (Rovick et al. 1996). However, besides the proof-of-concept study of a variable hardness socket with a novel multi-material AM process (Sengeh \& Herr 2013), the authors did not find much published recent information on AM socket fits. This is probably due to the limited mechanical properties of cost-effective AM, the low speed for fabrication or the high costs of high quality AM. To the best of our knowledge, there is no service on AM custom sockets to date.

Clinical studies of implanted AM prostheses are becoming increasingly popular. Aside from hip knee and spinal implants that continue to receive clearance from the U.S. Food \& Drug Administration ${ }^{39}$ (FDA) (Wohlers et al., 2016), the correction of maxillofacial (skull) defects is also becoming a clinical procedures. $\mathrm{Li}$ et al. reported the fabrication and implantation of a nanoscale hydroxyapatite/polyamide for the reconstruction of the jaw bone joint ${ }^{40}$ (Li et al. 2011). Further custom titanium restorations were implanted restoring nerve and artery channels in patients' skulls ${ }^{41}$ (Lin et al. 2013; Wang et al. 2012). These applications show that the possibility of fabricating porous materials is especially interesting for implantation as it accelerates osseointegration. This led to the commercialisation of OsterFabb, ${ }^{42}$ a process by Oxford Performance Materials. The company provides polymer implants that have FDA approval and clearance in the European Union. CEIT biomedical engineering received EU approval for AM made cranial, facial and jaw titanium implants and was able to negotiate costs of a recent treatment with the largest Slovakian insurer. ${ }^{43}$ One particular large-scale scull reconstruction was already performed and received broad media attention. ${ }^{44}$

The use of biodegradable materials also favour bone ingrowth. However, they degrade and are not detectable after a certain period of time. In this regard, Schantz et al. used a biodegradable polymer implant for cranioplasticity to close burr holes after surgery of a hematoma after traumatic brain injury. The implants got well integrated in the surrounding bone as could be seen in follow up CT scans after several months (Schantz et al. 2006).

The ability to tune the internal architecture provides additional opportunities to fit the implant to the mechanical properties of the anatomical site of interest (Wang et al. 2016). Together with the recent material and process approvals, AM of custom implants will likely become increasingly popular. However, Wang et al. also stress the lack of thorough long term implant studies, a missing database containing the mechanical and biological properties of human bones (depending on age, gender, location, and so on) and sophisticated software for topology optimisation and the design of internal structures as major limitations that, if overcome, might render AM a bigger share on the implant market. They also stress the software needs for the design of custom FO. As indicated in 2.2.1-2.2.3, the data preparation is a big issue in AM of biomedical devices. For the special purpose of creating FO, the need of processing drove research and industry to develop custom CAD software tailored for AM and named Personalised Orthotic Design (POD CAD). 45

The relevance of prosthetic technologies is an important topic in the discussions about human enhancement, also due to the fact that remarkable advances have been made in the development of such technologies.

While still many engineering challenges lie ahead, significant progress has been made in various areas of prosthetic technology in the present decade (and already before). This includes the area of limb

\footnotetext{
39 The FDA is responsible for protecting public health by assuring the safety, effectiveness, quality, and security of human and veterinary drugs, vaccines and other biological products, and medical devices in the U.S. https://www.fda.gov/AboutFDA/Transparency/Basics/ucm192695.htm.

40 Temporomandibular joint between the upper temporal bone and the lower mandibule

41 This work describes the reconstruction of traumatic zygomatico-orbital defects

42 http://www.oxfordpm.com/osteofab\%C2\%AE-products-0

43 http:/ / medicaldesign.com/design-engineering/additive-manufacturing-improves-cranial-implants

44 https:/ / 3dprintingindustry.com/news/3d-printed-cranial-implants-receives-eu-approval-36296/

${ }^{45}$ EU FP7 2298893 report on http://cordis.europa.eu/result/rcn/157437_en.html
} 
prostheses. The BeBionic prosthetic arm, for example, which already debuted in 2012, places sensors on the skin which, in conjunction with microprocessors in the arm itself, detects muscle movement from the remaining portion of the biological arm and translate patterns of movements into 14 different grips, which allow the user to perform several tasks, such as grip a pen, type on a keyboard or holding up to 100 pounds of weight (Niman 2013).

Suggestions that these instances of technological progress indicate the imminent emergence of 'cyborgs' who will vastly outperform "non-enhanced' humans are nevertheless highly questionable, mainly for two reasons: on the one hand, the technological state of art does not justify such statements, and, on the other hand, many users of prostheses explicitly reject extra functions, emphasising that they want a device as similar to a natural body part as possible.

The very scarce evidence of more widespread pro-enhancement attitudes appears to be limited to nonamputees. In an online delivered, exploratory and non-probability survey with non-injured members of a Canadian veteran group which was designed to explore whether veterans perceive the Canadian New Veterans' Charter as sufficient to ensure a full continuum of client-centred services and to gauge their perception regarding levels of satisfaction with service provisions, one question on human enhancement was included: "If you acquired an injury which led to the removal of your legs and you had the choice between a) artificial legs that behave like a 'normal' leg and therefore do not outperform 'normal' legs; b) artificial legs that that are better than the 'normal' legs and c) no artificial legs but something like a wheelchair, which would you chose?" (Wolbring et al. 2015). Of the 252 respondents which responded to this question, $153(60.7 \%)$ filled out the complete survey. As regards the question on human enhancement, $35.3 \%(n=54)$ expressed a preference for legs that outperform 'normal' legs.

We would like to emphasise that prosthetic technology is usually only applied for the compensation of congenital and injury- or diseases-related bodily limitations and in many aspects only allows for some compensation for the lost body part or function. However, future artificial limbs may comprehensively match the performance of natural limbs and could have intrinsic advantages (e.g., due to the used materials), and all such prostheses might in principle be designed with extra functions, including species-atypical ones. But only with regard to such more far-reaching visions of a future merger of humans and technology (massive cyborgisation of the human body), the potentials of additive biomanufacturing for human enhancement purposes appear to be significant.

\section{Key findings}

- Better fitting O\&Ps and unique aesthetic and wearable designs can be manufactured integrating joints, functioning and moving parts.

- Simpler manufacturing processes include property gradients, allowing weight reduction and reduction of material waste.

- Problems are the skills and experience required to obtain a good final product, limited reproducibility, high costs and limited mechanical properties.

- Foot and ankle-foot orthoses are the main AM O\&Ps; porous prosthetic implants are entering the clinic.

- An increasing number of FDA approvals for AM materials makes custom implants a promising field.

\subsubsection{Tissue engineering and organ printing}

Tissue engineering shows great promise for the regeneration of almost any tissue of the human body (Dhandayuthapani et al. 2011). It is an active area of research within regenerative medicine involving multiple disciplines including cell biology, biomaterial science, imaging and characterisation of surfaces as well as cell material interactions. It aims to restore, maintain or improve the function of defective tissues (Schneider et al. 2016). With respect to AM, the concept of tissue engineering refers to the placement of cell and/ or biologically active molecules on or within different matrices, the so-called 
tissue engineering scaffolds that shall promote the repair and/or generation of tissues. The scaffold itself is a cellular solid support comprising an interconnected pore network or matrix supporting cell colonisation, migration, growth and differentiation (Melchels et al. 2012; van Vlierberghe et al. 2011).

The design of these scaffolds poses a stringent technical challenge. The scaffold design mimics the natural extracellular matrix, a continuum with features ranging over multiple length scales, comprising multiple materials and cells and providing bioactive stimuli for tissue formation (Heller et al. 2009; Torgersen et al. 2013). They must fulfil a variety of functions. First, they provide cell-biomaterial interactions, promote cell adhesion and extracellular matrix deposition. Second, they shall permit certain transport characteristics such that gases, nutrients and regulatory factors reach the cells. Third, they need to be biodegradable at a controllable rate matching the rate of tissue regeneration under culture conditions (Langer \& Tirrell 2004).

With the development of biomaterials for AM, the automated fabrication of scaffolds with tuneable, reproducible and mathematically predictable physical properties has become possible. So far, cell free scaffolds, fabricated ex-situ and transplanted after their fabrication, have shown clinical success for bone , osteochondral tissue repair, cartilage and skin (Melchels et al. 2012). Clinical applications are mostly related to the fabrication of O\&Ps as reported above. The benefit of creating porous scaffolds favours osseointegration and hence tissue formation (Wang et al. 2016). Especially the use of FDM to create polycaprolactone scaffolds for bone tissue engineering is promising (Schantz et al. 2006). Commercial available interwoven meshes from Osteopore International ${ }^{46}$ (Osteomesh, Osteoplug) and their application as covers for burr-holes were already reported in 2.2.3 (Low et al. 2009).

Other polycaptrolactone and multimaterial scaffolds produced by FDM showed promising results for bone and cartilage tissue engineering in animal studies (Ho et al. 2010; Rai et al. 2010). A technology derivative called bioplotter was developed to create multifunctional and tailor-made scaffolds to create tailor made mechanical properties and gradients. ${ }^{47}$ Similarly, the Bioscaffolder from Systems Engineering comprises different dispense heads for gels and melt extrusion that provided higher seeding efficiency and more homogeneous cell distribution in vitro as compared to conventional techniques (Chen et al. 2011).

The inkjet printer technology has been explored to create controlled volumes of biological systems in a layer-by-layer fashion that is sometimes referred as organ printing (Boland et al. 2007). Cells and material can be deposited simultaneously. The interest in this approach led to the development of many robotic systems to create cell laden constructs mimicking various tissues. The ongoing commercialisation of these "bioprinters", the progress in tissue spheroid fabrication and the development of bioreactors is believed to open up new perspectives for organ printing. So far these matrices proved successful for cell studies including the development of blood vessels (Skardal et al. 2010), nerves (Marga et al. 2012), skin (Koch et al. 2012) and bone (Fedorovich et al. 2011). However, the approach is believed insufficient to produce large scale organs and is far from clinical trials (Mironov et al. 2011; Mota et al. 2015).

The main bottlenecks are the complication of mimicking the cellular organisation of natural tissues, the economically feasible upscaling to clinical applications and insufficient vascularisation of grafts (Melchels et al. 2012). Furthermore, anatomically correct models are difficult to fabricate, requiring 3D data files with clear information on soft tissue (Mota et al. 2015). Nevertheless, AM as a tool in tissue engineering seems promising in combination with other cell seeding processes. The prospect of digitally controlling distribution of material and cells in 3D, with high geometric complexity and accuracy, has made this field a very active research area, one that triggers excitement, scepticism and broad public

\footnotetext{
46 http:/ / www.osteopore.com/

47 https:/ / envisiontec.com/3d-printers/3d-bioplotter/
} 
discourse. Although media accounts ${ }^{48}$ already report that personalised organs will be printed in the future, a realistic short-term goal is the creation of models for drug testing (Faulkner-Jones et al. 2013)(Faulkner-Jones et al. 2015) or certain tissue components such as human heart valves (Cimetta et al. 2013).

The scale up, the available biomaterials and the regulatory hurdles, however, hamper a clinical application. AM tissue engineering remains mere a tool for studying cell behaviour and development in 3D.

In the scientific literature on tissue engineering goals for human enhancement are not mentioned. Although this field is described as very promising, the technology still faces many hurdles, such as keeping the larger printed structure stable under physiologic conditions and keeping the cells inside the structure alive, as described in the previous paragraph (see f.e. (Jana \& Lerman 2015); (Kang et al. 2016)). As far as we can see, AM plays no role in this context. While there are some voices which express far-reaching future visions such as printed organs exceeding the performance of natural organs, we did not find evidence of the use of additive bio-manufacturing taking place or being "in the pipeline" in this area. Likewise, Enno Park, a German expert from the do-it-yourself sector, whom we talked with for the project, does not know of any do-it-yourself activities in this area.

\section{Key findings}

- Tissue engineering depends on the placement and cultivation of cells on complex shaped matrices, the so called scaffolds.

- Scaffolds are supports made of interconnected pore networks mimicking the extracellular environment in a topological, chemical, and mechanical manner.

- Scaffolds are fabricated from biomaterials; they are tuneable, reproducible and have predictable physical properties.

- Fabricated ex-situ and transplanted into the body, they proved to be successful for bone, osteochondral, cartilage and skin tissue engineering.

- Using inkjet technology, discrete volumes of biological systems can be plotted in a layer-by-layer fashion, also known under the term organ printing.

- Several additive manufacturing tools for tissue engineering were developed and already commercialised.

- Problems are the complicated mimicking of the cellular organisation of natural tissues, upscaling that is not economically feasible and insufficient vascularisation.

- The availability of biomaterials is limited, regulatory hurdles exist.

- The field receives broad public attention and involves a large research area.

- It mostly remains a tool for studying cell behaviour and development in 3D.

\subsubsection{Drug delivery}

The digital control of matter gives AM the ability to create complex and personalised products, which also brings opportunities for arranging pharmaceutical ingredients to personalised drugs. Its use can have three main advantages: (1) the increase of product complexity as well as (2) low batch/patient specific and (3) on-site/on-demand production (Norman et al. 2017).

\footnotetext{
48 See, among others: Gallagher J. Doctors 3D-print 'living' body parts. Secondary doctors 3D-print 'living'body parts. 2016, http://www.bbc.co.uk/news/health-35581454; Whitman E. Well before it might produce hearts and livers, 3D bioprinting is reshaping modern medicine. 2016. http://www.ibtimes.com/well-itmight-producehearts-livers-3d-bioprinting-reshaping-modern-medicine-2364865: Zaleski A. Bioprinting: the new frontier in medicine that's not science fiction. 2015 http:/ / www.cnbc.com/2015/11/02/ bioprinting-the-newfrontier-in-medicine-thatmakes-human-tissue.htm
} 
Un-mouldable and difficult shapes can be produced including highly porous structures facilitating easy oral disintegration. These include gradients for tailored release of the active substance and the combination of active ingredients in one tablet allowing large freedom in product design. There are also a few publications on AM drug-device products, where personalised drugs overlap with surgical/ like wirelessly triggered capsules or antibiotic loaded catheters.

The first reported use of AM for the creation of a drug delivery device was already in 1996 (Wu et al. 1996). They specified the drug concentration profile in a CAD and report the release of two model dyes in a matrix of biocompatible polymers. The release could be altered either by the position or the composition of the dye in the matrix. Since then, several studies focused on the concept of tailored release and customised loading. The 3D printing technology seems particularly suitable for the creation of custom drugs. The vast majority of research is performed with this AM technology (Yu et al. 2008). The aims are to achieve zero order release (release independent from concentration) (Yu et al. 2007), pulsatile or timed release (Rowe et al. 2000) and fast disintegration (Goyanes et al. 2015). Further, implantable devices were studied that can perform a multitude of release functions for treating diabetes, for boned infections, for administering antibiotics, for immunisation and the like (Bhise et al. 2016; Gopalakrishnan et al. 2015; Huang et al. 2007; Inzana et al. 2015). The vast research efforts culminate into a recent report by Khaled et al. (Khaled et al. 2015). They investigated a 3D printed tablet containing multiple drugs with separated and defined release profiles. This shows that complex medication regimes can be combined and customised in a single tablet. Drug device products are also under development allowing for wirelessly triggered capsules (Gupta et al. 2015) or antibiotic loaded catheters (Weisman et al. 2015). Here, the research overlaps with tissue engineering. Researchers developed combination products allowing for pharmacologically controlled tissue engineering scaffolds (Xing et al. 2015).

Using AM, Chin et al. (2017) recently fabricated a drug delivery device that can be actively and wirelessly controlled after implantation without requiring an external source of power for the device. They present an implantable microdevice integrating movable biocompatible hydrogel patterns. It was promising in the treatment of osteosarcoma (bone cancer tumour) in a mouse model and potentially advances the development of implantable microelectromechanical systems without requiring device powering and related biocompatibility issues.

Apart from the tailoring of the release strategy, the potential of creating extremely low dose medication using highly potent drugs is also promising (Yu et al., 2008). AM allows for the economic production of personalised products at batch size one. Individual drug release products with personalised dosing can be made that likely benefit body mass and metabolism specific treatments, especially aiding therapies for growing children and those based on strong drugs (Norman et al. 2017; Vakili et al. 2015). It was proposed to integrate all components of a patient's daily medication into one cocktail (Khaled et al. 2014) that potentially takes into account the patients anatomy and metabolism (Sun \& Soh 2015). A study also focused on the acceptance of drugs fabricating different animal-shaped tablets for children. ${ }^{49}$

The additive fabrication of custom drug delivery devices may also come with a logistic advantage. The fabrication on demand can be especially useful for product development (Melocchi et al. 2015). In addition, this approach might make low stability drugs usable with limited shelf life. Li et al. described a method to perform digital synthesis on site ( $\mathrm{Li}$ et al. 2015). The research efforts on controlled release and tailored dosage led to the recent FDA approval of a 3D printed tablet ${ }^{50}$ with the name Spiritam, 51 an epilepsy drug for oral intake. Due to its porous structure, it readily disintegrates when taken with water. In addition, the drug content can be significantly higher and lower compared to conventional

\footnotetext{
${ }^{49}$ https://www.ucl.ac.uk/pharmacy/pharmacy-news/animal-shaped-tablets

50 http://www.accessdata.fda.gov/drugsatfda_docs/label/2015/207958s000lbl.pdf

51 http://icnapedia.org/explore/articles/news/entry/2015/11/24/spiritam-levetiracetam-world-s-first-3dprinted-drug-gets-fda-approval
} 
tablets allowing the adjustment to patients' specific needs. This may open the road for AM fabricated tablets, starting with treatments for neurological disorders. In January 2017, Renishaw received a 6 mill $€$ Horizon 2020 grant to support trials of a drug delivery system for Parkinson disease. ${ }^{52}$ The devices will be implanted into the skull of the patient close to the ear. The company does not specify the fabrication method of their drug delivery system, however, as Renishaw has long experience and various AM products, AM might also play a role in the fabrication of this novel system.

Despite these prospects, there is still limited experience with AM made drug release products. The solidification process in AM requires fusion mechanisms that may either involve incompatible chemistries or heat potentially hampering the drug's effectiveness and compliance requirements. Extensive process development, testing, the development of standards and regulations for AM drug release products hamper their broader acceptance and use thus far. AM drug delivery products first have to compete against the established regulatory pathway in place for conventionally manufactured drug products.

Personalised medicine as a way of delivering medicines to individuals in a safe and effective manner relies on diagnostic/genetic testing and targeted drug delivery which require a new manufacturing method, capable of producing products in small numbers (Yin et al. 2010). 3D printing in pharmaceuticals has the potential of designing simple, accurate, cheap, structured and tailored drug delivery systems, since 3D structures can be printed on a functionalised surface characterised by a specific permeability, porosity, hydrophobicity/hydrophilicity or functionality (Goole \& Amighi 2016). In their review study Goole \& Amighi (2016) conclude that modern pharmaceutics finds itself a turning point due to these advancements. The advances biotherapy and personalised medicine which have bought to novel concepts of formulation of drugs, such as nano-scale medicines, biomimetic particles, functionalised liposomes, as well as to more sophisticated manufacturing methods, have been also associated with the potential of creating drugs beyond therapeutic purposes. There are different techniques concerning the 3D production of pharmaceuticals, both with their pros and cons, and they still have to overcome the regulatory approval process. Therefore, since the application of 3D printing for drug delivery is still at an early stage, there is no scientific literature on the potential for human enhancement applications.

\section{Key findings}

- The field involves low batch and customised production, production on-site and on-demand.

- Difficult designs for easy oral disintegration and tailored and/or triggered release mechanisms are often unmouldable.

- Capsules containing multiple drugs for multiple treatments become possible.

- Individual dosing may be based on the respective body mass and metabolism.

- AM techniques enable treatments on low stability drugs on-site.

- Experience with AM made drug release products is limited.

- Many fusion mechanisms in AM are incompatible with many drug chemistries.

- AM products meet problems with the regulatory pathway in place to date.

\subsubsection{Food printing}

The first-generation food printer concept designs were introduced to the general public more than 10 years ago but they had a very modest diffusion: Nanotek Instruments, Inc., patented a rapid prototyping and fabrication method for producing 3D food objects, such as a customer-designed birthday cake; but it did not build any physical prototype (Lin et al. 2001). In the Electrolux Design Lab 2009 competition Electrolux came out with a Moléculaire concept design, which could print a multi-material customised

\footnotetext{
52 http:/ / www.renishaw.com/en/horizon-2020-grant-supports-trials-of-a-new-renishaw-drug-deliverysystem-in-the-treatment-of-parkinsons-disease--39926
} 
meal using a small robotic arm. Between 2009 and 2010 a few food printing projects were carried out (Cohen et al. 2009) (Hao et al. 2010; Lipton et al. 2009), mainly focusing on the fabrication of customised food items as Sun et al. reported (Sun et al. 2015b). More recently Netherlands Organisation for Applied Scientific Research (TNO) and Barilla (Italian pasta company) have presented the preparation of 3D printed pasta using classical pasta recipes (ingredients: durum wheat semolina and water, without additives) ${ }^{53}$. A company called Natural Machines created Foodini Food printer which extrudes fresh food ingredients to design meals, using these ingredients for surface filling (e.g., pizza or cookie dough and edible burger from meat paste) and graphical decoration.

Food printing is a digital food fabrication process integrating 3D printing and digital gastronomy technique to manufacture food pieces. The current food printing process starts with designing a virtual 3D model. Then slicing software translates this model into individual layers and finally generates machine codes for printing. After uploading the codes into a printer and choosing a preferred food recipe, the food printing starts. Sun et al. (2015a) define a food printing platform as consisting of an XY-Z three axis stage (i.e., a Cartesian coordinate system), dispensing/sintering units, and a user interface. With a computer-controlled material feeding system, such platforms can manipulate food fabrication in real time. The food printing process does not require a high-energy source to completely remove liquid ingredients from food composition, since fabricated layers do not need to be completely solidified, but they require sufficient rigidity and strength to support its own weight and the weight of subsequent layers without a significant deformation or shape change. Following Sun et al. food printing "allows users to design and fabricate food with customised colour, shape, flavour, texture, and even nutrition. As a result, our eating experiences can go beyond taste to encompass all aspects of gastronomy, such as food preparation, culture, economy, physics, and chemistry" (Sun et al. 2015b).

Some 3D food printers are already in use in commercial settings and nursing homes, while others in the development and experimental phases and some remain at the speculative stage. It was reported that 3D printed food is currently served in more than 1,000 German nursing homes to residents who have difficulty chewing 54 . This food is described as positively accepted by residents since it is more appetising than traditionally pureed food. The available materials for food printing can be classified into three categories: natively printable materials (like hydrogel, cake frosting, cheese, hummus, and chocolate), non-printable traditional food materials (such as rice, meat, fruit, and vegetables), and alternative ingredients (such as the ones extracted from algae, fungi, seaweed, lupine, and insects).

3D Food Printing Technologies include Selective Laser Sintering and Hot Air Sintering (laser and hot air can be utilised as a sintering source to fuse powder particles and form a solid layer); Hot-Melt Extrusion (which has been used to create customised 3D chocolate products, (Hao et al. 2010) and Room Temperature Extrusion (which has been applied to natively printable materials like cheese, frosting, and hummus); Binder Jetting (each powder layer is distributed evenly across the fabrication platform, and a liquid binder sprays to bind two consecutive powder layers) and Inkjet Printing (which dispenses a stream of droplets from a syringe-type print head in a drop-on-demand way for cookie, cake, or pastry fabrication).

The cost of 3D printed food products includes expenses associated with printing platforms (hardware and software) and printing materials, labour cost, operation cost, and general overhead for maintaining the production facility. Sun et al. reported that the current price of commercial food printing platforms is at least a few thousand dollars, still too high as a consumer product in terms of fabrication capabilities (Sun et al. 2015b). In addition, consumers need to purchase printing materials from the platform companies which are more expensive than that of the similar materials in the market (Sun et al. 2015b). Sun et al. conclude that food printing is an emerging and promising field which needs the development of a systematic way to investigate recipes, platform design, printing technologies, and their influences

\footnotetext{
53 https:/ / ec.europa.eu/jrc/sites/jrcsh/files/20150225-presentation-jan-sol.pdf

${ }^{54} \mathrm{http}$ :/ / www.cbsnews.com/news/nursing-homes-in-germany-serve-3d-printed-food/
} 
on food fabrication: "Meanwhile, the food design process should be structured to promote user's creativity, the fabrication process should be quantified to achieve consistent fabrication results, and a simulation model should be developed to link design and fabrication with nutrient control" (Sun et al. 2015b).

In June 2016 social researchers in Australia conducted one of the first studies on potential consumers' attitudes toward 3D food printing (Chen et al. 2017). Lupton and Turner ${ }^{55}$ analysed responses to an online discussion group with 30 Australian participants that examined these issues over a four-day period in March 2016 (Lupton D.). The participants were asked to respond to a new set of questions each day. During this period, they were able to return to previous days' questions and answers and change, add new responses or elaborate their responses. Almost all the participants were unfamiliar with 3D printing. They elaborated strong affective responses to what they considered to be unnatural elements of the 3D printing process and the food it generated (food would be somehow 'plastic' and therefore inedible). The group was in general not so much concerned with environmental issues related to food: it was then not a surprise that the potential environmental benefits of $3 \mathrm{D}$ printed food were rarely mentioned. They also made little explicit mention of the potential environmental sustainability contribution and the contribution to alleviate world hunger by using alternative food sources such as alga or insects, also for 3D printing. The majority saw these ingredients as disgusting and they were concerned by the physical appearance of this food. In general, the majority of the participants expressed scepticism about the use or value of 3D food printing technologies for their own lives. They were generally not willing to introduce this food in their diets. All in all 3D food fabrication raised four different types of concerns: the first related to the way in which these substances are processed and prepared; the second has to do with the lack of understanding of the fabrication process, which sounded exotic and strange for people; the third has to do with the appearance of the objects that are generated by this technology: in many cases foods look very different from familiar one; the fourth has to do with the unfamiliarity of the content of the food produced by 3D printing, which contain something different such as the ones produced from algae or insects as well as in vitro meat. Lupton and Turner (2016) see their results as confirming the studies previously quoted by Razin and his colleagues ${ }^{56}$ (Rozin et al. 2012) concerning the importance of 'natural preference' in shaping people's responses to novel food technologies, with some caveats. Lupton and Turner (2016) ${ }^{57}$ argue that the scepticism has to do with the lack of knowledge and understanding of the attribute of process of food fabrication in part due to the title of the technology, since the word 'printer' is traditionally a technology associated with the use of chemicals to make marks on surfaces. Indeed, even those who knew something of 3D printing in general, associated the process with manufacturing objects such as toys from plastic substances, something very different from food. They conclude: "Our findings suggest that efforts to promote use of these technologies to achieve key activist goals must engage with the persistence of a consumer preference for 'natural foods' and the importance of their immediate affective and visceral reactions to food, including such elements as what kind of technology is used to process it, what the food is perceived to be made of, how fresh itis, the degree of processing it undergoes and how many other elements may be added to create it. [...] Our research findings suggest that to maximise the potential benefits of 3D food printing for activism, there is a need to shift the focus of the technology beyond niche groups (such as the elderly, the poor and those living in areas where food supplies are limited) and to emphasise its qualities for better nutrition and the positive dimensions of its novelty" (Lupton and Turner 2016, p. 1558).

The new research published by Meat \& Livestock Australia (MLA) in May 2016 indicated 3D printing technology as the next big advancement for red meat products (Meat and Livestock Australia Limited

\footnotetext{
55 https:/ / papers.ssrn.com/sol3/papers.cfm?abstract_id=2799191

56 https://edibleschoolyard.org/sites/default/files/meaningfoodinlivesJNEB2005.pdf

57 https:/ / papers.ssrn.com/sol3/papers.cfm?abstract_id=2799191

58 https:/ / papers.ssrn.com/sol3/papers.cfm?abstract_id=2799191
} 
2016). Meat can be used as a raw material in different forms and different processes, especially powderdried meat (Godoi 2015). In particular, the report written by researchers of the University of Queensland for MLA Australia state that the meat industry has potential to play an important role on the consolidation of this technology because of the rich protein content in the muscles combined with the variety of triglycerides and mineral contents (Meat and Livestock Australia Limited 2016). There are four major advantages in the use of 3D printing technologies for red meat products: reduction of waste per animal carcase; additional volume of meat sold; additional value per carcase and reduced cost of processing, capital and running cost.

In vitro meat (IVM) is meat grown in a culture medium using the techniques of tissue engineering, instead of being taken from living animals. In the first step, muscle cells are taken from an animal through a muscle biopsy and from these cells skeletal muscle stem cells, also known as satellite cells (SATs), and adipose tissue-derived stem cells (ADSCs) are extracted. Then these cells are grown on a petri dish with the help of a growth-medium. In this way, the muscle cells are able to assemble forming muscle fibres and then muscle tissue in a so-called bioreactor (Post 2014). Once the muscle fibres are harvested, they are assembled into a patty to create different products, such as hamburger or meat balls. Other compounds could be added either to make the patty more similar to that of conventional meat or to increase the nutritional value of the product. In this case, IVM could also be developed as innovation in the field of functional food.

Although the idea of IVM was first imagined in 1927 by J.B.S. (John Burdon Sanderson) Haldane and, more concretely, in 1931 by Winston Churchill and was materially researched in laboratories of NASA in the 1950s, this research became possible later in the Nineties through the development of different branches of biology, namely stem cell biology and tissue engineering. The first patent for a method producing in vitro meat is dated 1997. Five years later, the first in vitro grown tissues were created by two different groups: a scientist group at NASA created fillets of goldfish muscle tissue and announced it in a scientific publication (Benjaminson et al. 2002); a bioart group presented muscle tissues grown using foetal sheep cells in different exhibitions with the intent to invite the public to reflect on the implications of using medical technologies for non-medical needs (Catts \& Zurr 2014).

Both these activities remained largely unnoticed by the general public. It was then first through activities of engaged scientists, charities and private investors, that the idea of producing meat through the means of tissue engineering has become a vision attached to a particular message concerning sustainability, ethics and future food (Ferrari \& Lösch 2017). A turning point in the history of IVM development was the presentation of the first in vitro meat burger made entirely of bovine stem cells by Mark Post in London on August the 5th 2013 at a hybrid science-media event (something between a cookery show, a press release, and (Ferrari \& Lösch 2017) an experiment. This event was introduced by an explanatory short film created by the media production company The Department of Expansion (still visible on youtube), which served also to launch the project "cultured beef" at the University of Maastricht.

At the moment an industrial production of in-vitro meat is not possible: the products which have been until now presented by scientists or some start-up companies, such as the previously described burger created by Mark Post and his group, the meat balls created in January 2016 by the company Memphis Meats, are prototypes and not ready for the market. In March 2017 Memphis Meats announced the creation of the first lab chicken and duck meat ${ }^{59}$. In the IVM development process there are still different technical hurdles, such as (1) finding cells with a large capacity for multiplication; (2) the formulation of culture media to ensure a high growth rate of cells (3) use of a biomaterial that helps cells to organise themselves in a tissue configuration; (4) the design of a large-capacity bioreactor to feed the cells correctly in optimal conditions which allow their differentiation and therefore the large-scale IVM

\footnotetext{
59 http:/ / www.sciencemag.org/news/2016/08/lab-grown-meat-inches-closer-us-market-industry-wonderswho-will-regulate
} 
production as well as (5) the production of muscle tissue which resembles conventional meat as much as possible (especially concerning the colour, the taste and the consistency (cf. (Bonny et al. 2015). Besides these technical hurdles, there are economic, epistemic and ethical problems connected to the use of growth medium for cells: Until now the most efficient growth medium is foetal calf serum, a medium very well known in the field of cell cultures, which is obtained by a painful procedure from the blood of aborted calves. This serum is difficult to standardise, it is proven to be able to transmit diseases and it is extremely expensive. IVM innovators seem to be very well aware of the limitations and are engaged into finding animal-free alternatives to the growth medium. Post and his team have identified some of serum-free culture conditions promising for SATs and ADSCs starting from algae lysates (Hocquette 2016; Wu et al. 2014)

Interestingly enough, one of the leaders of 3D bioprinting for toxicological assays is Dr. Gabor Forgacs George H. Vineyard Professor of Biological Physics at the University of Missouri-Columbia, Scientific Director of the Shipley Center for Innovation at Clarkson University, scientific founder of Organovo, Inc. and also founder of Modern Meadow, Inc. Modern Meadow is a leading start-up company in the field of in-vitro meat and in-vitro leather. Forgacs uses a bioprinting technology which lays down bioink units - a preparation of multicellular aggregates - with support materials along an architectural template. In a conference organised by New York Academy of Sciences, Forgacs explained that the structural formation occurs post-printing, in a process similar to embryonic development of animals (Wu et al. 2014). Despite the different technical hurdles IVM innovators believe that because of dwindling natural resources and global population growth, IVM might be not just a choice, but rather a necessity in the future (Wu et al. 2014).

The potential of food production and printing for human enhancement concerns the previously mentioned field of functional food or personalised nutrition (Watzke \& German 2009). Here the difference between "usual" potential of food production and printing and functional food is not very clear, since some authors, such as for example (Sun et al. 2015b) describe the entire field of 3D food printing as a technology which can produce food with customised shape, colour, flavour, texture and even nutrition.

As regards potentials of 3D printing for realising personalised food (i.e. food targeted to the individual needs of the consumer), fairly often discussed examples are products high in iron targeted to female consumers, especially pregnant women, and products high in protein targeted to kids or athletes. Furthermore, dietary malnutrition of the elderly in aged care facilities has been addressed in this context: "Because the competing product (pureed food) has a low score in appearance and texture, 3D printed food presents a unique opportunity to overcome the barriers of this segment and offer a real consumer benefit" (Meat and Livestock Australia Limited 2016). This is particularly important in the therapeutic context of keeping patients with dementia alive.

The possibility to add different nutrients to the constructed and printed food, as well as different properties have been part of the debate on IVM since the beginning, but only at the margins. IVM innovators in this context often emphasise that their products will not contain any form of genetic engineering.

Even when using a broad notion of human enhancement, the current relevance of 3D printing for human enhancement uses appears to very low in this area too, and the potentials are unclear, at best.

\section{Key findings}

- Digital fabrication processes integrate 3D printing and digital gastronomy.

- Customised colour, shape, flavor, texture and nutrition can be produced from a digital file.

- Commercial food printers are available for nursing homes.

- Costs are too high for commercial use.

- Natively printable substances (such as hydrogels, cake frostings, cheese, ...) stand against non printable traditional materials 
- An industrial production of in vitro meat is still not possible due to technical hurdles.

- In vitro meat can contribute to tackle the challenge of climate change in the food production.

- Public acceptance of in vitro meat remains unclear. 


\section{Socio-tec hnical imaginaries}

There has been growing recognition (Bouchard 2003) in the social sciences (Taylor 2004) and humanities (Castoriadis 1997) that imagination is a crucial, constitutive element in social life. Beyond representations and actions, human beings produce symbols. Mental contents are symbolic in nature, because they bridge to reality, but they are not the reality itself ${ }^{60}$. Symbols are multiverse; they link to reality but also to other mental contents. This implies that symbols are always overdetermined, say, they convey extra, implicit, meanings. Webs of meanings are stored, communicated to, and absorbed from, other humans through narratives (McComas \& Shanahan 1999), both personal (e.g., life stories, dreams, fantasies) and collective (e.g., legends, myths, artistic expressions).

The concept of socio-technical imaginary (Marcus 1995) has been developed to provide an analytic framework for exploring the role of narratives in the production of new technology. People embed in stories the way in which they perceive technology innovation. The importance of these narratives is twofold, on one hand they provide significant clues about how society, and social groups, are metabolising technology innovation, enlightening fears, hopes, expectations and fantasies. On the other hand, they provide social actors with models and points of view to inform their technological choices. Today, it is apparent that economic drivers, and rational arguments, are not sufficient to explain increasingly complex innovation dynamics. The more techno-science is a collective enterprise, the more imaginary dimensions become significant ${ }^{61}$. One of the most established methodologies to explore the sociotechnical imaginary is through science fiction (SF or sci-fi) ${ }^{62}$.

Although many gadgets and inventions have been predicted by $\mathrm{SF}^{63}$, what make it relevant to technology policy setting is not that it might allow predictions, but that it provides precious information on interactions between technology innovation and society. As Frederik Pohl - American science fiction writer, editor, and lecturer - once said "A good science fiction story should be able to predict not the automobile but the traffic jam". Scholars have proposed to use SF as a prototypical framework to test technology innovation and its societal impact. Science Fiction Prototyping (SFP) is today an established methodology (Potstada \& Zybura 2013). SFP goes beyond the analysis of existing narratives to draw upon for alternative future worlds, being technique to generate scenarios. Johnson (Johnson 2011), who coined the term, describes SFP as "stories grounded in current science and engineering research that are written for the explicit purpose of acting as prototypes for people to explore a wide variety of futures".

3D Printing is largely present in SF, in novels, movies, TV series, video games, and comics (Birtchnell \& Urry 2013). In a recent study (Stein 2017) Adam Stein has investigated political imaginaries related to 3D printing discourse He argues that there are three key themes, which emerge from the analysis of narrative and literature surrounding 3D printing,

1. Maker-as-entrepreneur: 3D printing as an enabler for the individual maker as a neoliberal entrepreneur in a world of capitalist opportunity;

2. Economic revival of the nation state: 3D printing as a boon to productivity through the "on shoring" of manufacturing, enabling national economic revival, particularly in the Global North;

\footnotetext{
60 This concept is nicely expressed by Magritte's famous painting "The Treachery of Images", which shows a pipe; below it, Magritte painted, "Ceci n'est pas une pipe" ("This is not a pipe").

${ }^{61} \mathrm{http}: / /$ sts.hks.harvard.edu/research/platforms/imaginaries/iii.proj/nsf-summary-and-proposal/

62 Science Fiction is "a contemporary mode in which the techniques of extrapolation and speculation are utilised in a narrative form, to construct near-future, far-future or fantastic worlds in which science, technology, and society intersect" (Thacker 2001).

${ }^{63}$ Many SF predictions, however, turned out being wrong.
} 
3. Commons-based utopia: 3D printing as a social tool in a post-capitalist, commons-based utopia featuring material abundance, shared resources, and widespread community control over the means of production.

The first theme (Maker-as-entrepreneur) portrays 3D printing as an enabling technology, which could contribute to produce a new generation of individual entrepreneurs, as in a new "Silicon Valley Revolution". In this scenario, a wave of novel entrepreneurs would exploit 3D printing to create new opportunities for all, through the flourishing of a myriad 3D printing based companies. This theme is defined in political terms by Stein as a neoliberal utopia, set in "in a world of capitalist opportunity".

The second theme (Economic revival of the nation state) depicts 3D printing as an opportunity for promoting manufacturing at local level, contrasting globalisation and delocalisation processes. This is a scenario in which local companies react to globalisation and delocalisation by developing 3D manufacturing. This theme is defined by Stein as "national economic revival".

The third theme (Commons-based utopia) describes 3D printing in terms of "commons-based utopia featuring material abundance, shared resources, and widespread community control over the means of production". It is a "post-capitalist" scenario, characterised by a new notion of manufacturing, which would deeply transform the market based economy by establishing a new era of material abundance at almost no cost. This is certainly the most utopian and visionary theme in Stein's taxonomy. In practice, 3D printing is conceptualised as a powerful driver of social, economic, and political transformation, which would eventually overturn most current standards.

It is important to emphasise that Stein considers these three themes as three imaginary answers to current worries and anxieties connected to automation, globalisation, and vanishing of traditional manufacturing jobs. In Stein's view, they do not represent predictions, or forecasts, about future 3D manufacturing, rather they unravel present hopes and fears related to 3D printing. The importance of knowing and mastering them mainly dwells into their nature of fantasies shaping public attitudes and, in the same time, into their capacity for driving technology development by providing collective visions.

\subsection{Cultural imaginaries of 3D printing}

Stein's analysis is thought-provoking and, to some extent, it is also convincing. Yet, Stein is chiefly interested in political and socio-economic narratives. The wider societal and cultural perspective lies beyond his focus. In the present study, we are instead more interested in exploring the "human" dimension of 3D printing, including worldviews, perceptions, rights, choices, ethical and cultural issues.

We have scanned the main science fiction online databases, including The Internet Speculative Fiction Database ${ }^{64}$; The Science Fiction and Fantasy Research Database ${ }^{65}$; Risingshadow ${ }^{66}$; Technovelgy ${ }^{67}$; SF Site ${ }^{68}$; Free Speculative Fiction Online ${ }^{69}$; Internet Movie Database70; Fantastic Fiction ${ }^{71}$; Feminist SciFi72; SciFi Movie

\footnotetext{
${ }^{64}$ http:/ / sffrd.library.tamu.edu

65 http://www.sfadb.com

66 https://www.risingshadow.net

67 http:/ / www.technovelgy.com

68 https://www.sfsite.com

${ }^{69} \mathrm{http}: / /$ www.freesfonline.de

70 http://www.imdb.com

71 https://www.fantasticfiction.com

$72 \mathrm{http}$ // feministsf.org
} 
Pages ${ }^{73}$; SF Booklist ${ }^{74}$; Ultimate SF Web Guide ${ }^{75}$; The Internet Movie Script Database ${ }^{76}$; TV.Com ${ }^{77}$ We have searched for "3D printing", "3D printer", "Additive Manufacturing", "3D Manufacturing", "bio printing", "bio manufacturing", "human duplication", "body modified humans" searching for novels, short stories, movies and video games. In total, we have identified 149 titles, of which 46 turned out being relevant to the present study. They cover a time ranging from 1930 (The Cosmic Express by Jack Williamson) ${ }^{78}$ to 2016 (Greg Bear's Killing Titan) ${ }^{79}$.

We have analysed these stories by using the scheme proposed by Bal (Bal 1997), which is based on three level layer of analysis: 1) the Fabula, the series of events that are being represented, say, the archetypal stories; 2) the Story, which is a subset of the Fabula restructured into a meaningful sequence for a particular effect, say, the storyline; 3) the Narrative, which is the story given form with all the added semantics of the form itself, for example a monologue, novel, film or multimedia presentation, etc.. Our investigation started with a collection of narratives (in form of novels, short stories, movies, and games), from which we have extracted a few storylines. By analysing and comparing these storylines, we have finally identified three main fabulae, say, archetypal stories 1) the Pandora's box; 2) the Cornucopia; and 3) the Magic Bag.

\subsubsection{The Pandora's Box}

The archetypal story of Pandora's Box ${ }^{80}$ is based on two main elements, 1) a "holy" container, a jar in the original Greek myth; 2) something which must be confined inside, because it is gifted with an exceeding power (evils and hopes, in the original myth). By accident, hubris, or stupidity, the items hold by the container are freed; so, they invade the world, threatening horrible disasters. Pandora's box plot is in the background of most narratives surrounding 3D printing, notably those connected to human organs, and full body, printing. When 3D printing is used for "producing" human bodies (or parts of them), the myth of the Pandora's box is frequently implicitly evoked by SF literature. This is quite evident in SF films like the Fifth Element (1997) (Figure 10) ${ }^{81}$, where the artificial production of a new human being is due to a powerful "Fifth Element" (whose exceeding power must be contained) and in novels such as The Printer's Devil by Chico Kidd (1996) ${ }^{82}$, where the "Printer" is animated by a powerful, devilish, force (somehow echoing Stevenson's 1891 short story The Bottle Imp). Stephenson's 2015 novel Seveneves (Stephenson 2015) tells the story of the efforts to preserve human society after an apocalyptic event (the unexplained disintegration of the

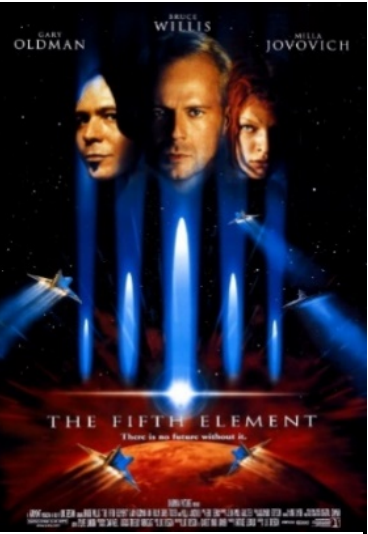

Figure 10 The Fifth Element. Source: Wikipedia. https://en.wikipedia.or g/wiki/The_Fifth_Eleme nt. Moon). These efforts include the creation of a sort of Noah's ark (the "Cloud Ark"), where saving a Human Genetic Archive, to be used to rebuild the human population. The Cloud Ark exploits 3D printers to replicate genetic material and to produce a variety of robots. Unfortunately, the "Cloud Ark" turns soon into a claustrophobic scenario 83 , where the most its population die of various causes. Finally, only eight survivors, all female, remain alive. The Human Genetic Archive has been destroyed, but they have sufficient resources to use the Ark's genetics laboratory to create genetic

\footnotetext{
73 http:/ / www.scifimoviepage.com

74 http://www.sfbooklist.co.uk

75 http:/ / www.magicdragon.com/UltimateSF/SF-Index.html

76 http:/ / www.imsdb.com

77 http://www.tv.com/shows/category/science-fiction/

78 http:/ / www.goodreads.com/book/show/6328997-the-cosmic-express

79 http://www.goodreads.com/book/show/24819460-killing-titan

80 https://en.wikipedia.org/wiki/Pandora\%27s_box

81 https://en.wikipedia.org/wiki/The_Fifth_Element

82 https://www.amazon.com/Printers-Devil-Chico-Kidd/dp/1935961195

83 The Ark is a closed Pandora's box, a trap, apparently full of hopes but instead full of evils.
} 
printers that could rebuild the human species by parthenogenesis. The narrative then continues in the far future by telling the story of the eight human races originated from the eight survived women.

The Pandora's box myth also informs the recent wave of SF dystopian novels and movies based on the hypothesis of technological singularity, say, the hypothesis that progress in artificial intelligence and/or enhancement technologies might lead to the emergence of a posthuman race, either biotechnologically modified transhumans or software-based synthetic minds, which threats to supersede the human species (Eden et al. 2012). The plot of most of these stories includes the element of a container (e.g., a lab, a special glass cell, a quantum computer memory, even a Faraday cage, etc.) which fails to contain a powerful technology, which eventually escapes and threats the human species. One could mention for instance, the 2016 British-American SF horror film Morgan ${ }^{84}$, where a powerful human hybrid created by biotechnological printers, which can replicate genetic material through 3D-printing of the zygote or the sperm and the egg - escapes from the glass cell in which it is confined; the 2014 Warner Bros production Transcendence ${ }^{85}$, including Hollywood stars like Johnny Depp and Rebecca Hall, in which the same manufacturing technology produces both nano-particles, which might eradicate pollution, disease, human mortality; and computer viruses, which might destroy the whole technological civilisation. In the role-playing game Eclipse Phase ${ }^{86}$, progress in AI and nano-fabrication allow people printing at home everything they need from the molecular level; these printers are also used to enhance and clone human bodies; and to digitalise minds and memories, making it possible to transfer them over long distances and download them into new bodies (biological or synthetic). Such a utopian scenario is soon disrupted by a group of out-of-control AIs, the Titans, probably generated by a virus escaped from the printing devises. Titans transport themselves through a network of wormhole gateways, known as "Pandora Gates" ${ }^{87}$. These examples - just to mention a few - illustrate quite well one of the most significant features of the imaginary surrounding 3D manufacturing. Future manufacturing techniques and devises are imagined as very powerful tools, which could beneficially overturn our society, by manipulating human bodies and minds in the same way in which they can manipulate material items. Yet, such an "objectification" of our humanity entails the inherent risk that this technology could also generate a mortal threat to human survival. There is indeed a dark, even macabre, side in the Pandora's box myth, well showed by equivalent legends in Celtic, Norse and Welsh mythology. In these legends, there are often magical cauldrons ${ }^{88}$, which play the same function of the Pandora's box in Greek mythology. Yet, these cauldrons had also a further function, they could swallow, digest and revive corpses. This would echo ancient Neolithic religions associating the coffin and the earth with the maternal womb, according to the hypothesis first proposed by Lithuanian-American archaeologist Marija Gimbutas (Gimbutas \& Gimbutas 1991), who pioneered feminist archaeology.

\subsubsection{The Cornucopia}

The Cornucopia is the second archetypal myth shaping the collective imaginary on 3D printing, as it emerges from SF narratives. In Greek mythology, the cornucopia is a marvellous horn, pouring an unceasing abundance of ambrosia and fruit. Almost all cultures have examples of myths based magical dispensers, e.g., the Pot of Gold in Irish mythology ${ }^{89}$; the Magic Tablecloth in Russian folklore ${ }^{90}$; the Crock and Dish of Rhygenydd Ysgolhaig, in Welsh mythology ${ }^{91}$; the Akshaya Patra, in Hindu mythology ${ }^{92}$; the

84 http://www.imdb.com/title/tt4520364/

85 http://www.imdb.com/title/tt2209764/

$86 \mathrm{http}: / /$ eclipsephase.com/game

87 The name given to these gateways clearly show the source of the original inspiration behind the Eclipse Phase's plot

88 https://en.wikipedia.org/wiki/Cauldron

${ }^{89} \mathrm{~A}$ pot full of coins at the end of the rainbow.

90 A tablecloth where food and drink magically appear.

91 Jar and plate where food magically appeared.

92 A vessel, which provides a never-ending supply of food. 
Manna Machine, in Jewish mythology ${ }^{93}$. The Cornucopia basic plot is likely to be the most diffused archetypal SF story on 3D printing.

The two chief examples are likely to be the Matter Compiler in The Diamond Age (Stephenson 1995) (Figure 11), by American writer Neal Stephenson ${ }^{94}$; and the Replicator and Bio-matter Re-sequencer, in the Star Trek saga.

The Matter Compiler is a machine which can directly assemble atoms. This machine can create all sort of materials and devices atom by atom, using only carbon, oxygen, nitrogen and chlorine. In major cities, there are public matter compilers, which provide the people with basic food and water for free. They receive molecules from a public grid - like today electrical grid - called the Feed. This grid is controlled by a restrict oligarchy. In opposition to the Feed, there is an open, decentralised, distribution system, called the Seed, which is run by various anarchical and independent networks. The Matter Compiler is an interesting mix between a Cornucopia- based scheme and the Stein's Commons-based utopia theme., the existence of a machine, which produces food for free, does not contribute to generate a post-Capitalist society (as in Stein's hypothesis) rather it produces neo-feudal hierarchies.

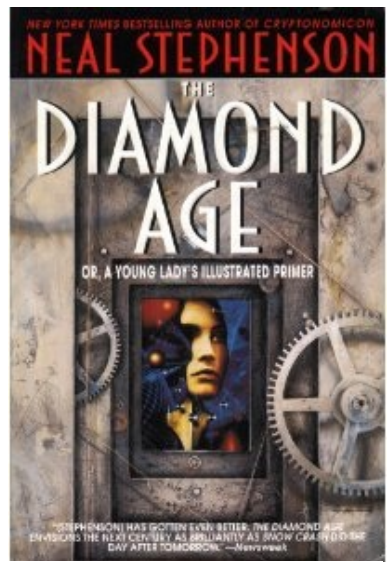

Figure 11 The Dimond Age. Source: WKIPEDIA. https://en.wikipedia.org /wiki/The_Diamond_Ag e.

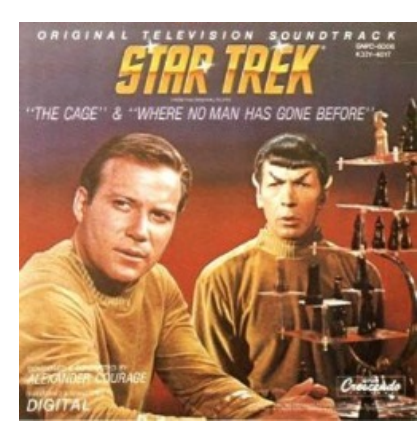

Figure 12 Star Trek. Source: WIKIPEDIA. https://en.wikipedia.org/ wiki/Star_Trek.

In Star Trek TV series ${ }^{95}$ (Figure 12), there is machine, which produces usable material from waste. The device can dismantle any object into subatomic particles and re-assemble them into new objects. In the 1966 original series, this Replicator was chiefly used to provide food and water on board star ships. In various spin-off series, the replicator machine has progressively acquired many further functions, including 1) energy production; 2) spare part production, making it possible to repair star ship damage without having to return to a base; 3) replication of starfleet uniforms; 4) production of food, clothes, and other everyday objects. There is also a safety protocol, which prevents replication of dangerous objects, such as weapons and poisonous substances. Finally, the Replicator has become a veritable magical dispenser, which can produce any kind of item and provide any kind of service, turning anything into anything else. Yet, there is a bug in such a wonderful device. In the episode Call to Arms ${ }^{96}$, the replicator is used to produce weapons (mines) that have a replicator inside, say, they can self-replicate. Although this theme is not developed, it is evident that it introduces in the Replicator narrative an ambiguous and threatening element, self-replication, developed by Michael Crichton in Prey (Crichton 2002).

\footnotetext{
93 A machine described within the kabalistic text, Zohar

94 https://en.wikipedia.org/wiki/The_Diamond_Age

95 https://en.wikipedia.org/wiki/Star_Trek

96 http://memory-alpha.wikia.com/wiki/Call_to_Arms_(episode)
} 
In Transmetropolitan (Figure 13), a cyberpunk transhumanist comic book series, written by Warren Ellis ${ }^{97}$, published from $1997-2002$ by DC Comics, there is an invention called the Maker. By taking a block of base materials, and after receiving a simple vocal command, these devises can make anything, food, clothes, weapons, drugs and so. Each morning, Makers sweep the streets for garbage, gathering the matter to recycle it into more useful objects.

In Planetfall (Newman 2015), a novel published in 2015 by Emma Newman, the main character is a 3D printing engineer, working in a space colony, creating the tools necessary for human survival in an alien environment. In this novel, $3 \mathrm{D}$ printing is described to produce food and basic materials, which might allow humans to survive in hostile conditions.

Similarly, 3D printers are an essential element also in 2014 Cory Doctorow's novella, The Man Who Sold the Moon ${ }^{98}$. Cory Doctorow - science fiction author, former European director of the Electronic Frontier Foundation and cofounder of the UK Open Rights Group - imagines a near future where a group of parkourist hackers crowdfund open-source, autonomous, 3D printers, which

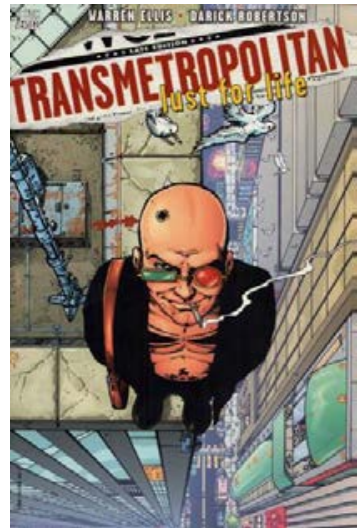

Figure 13 Transmetropolitan. Source: WIKIPEDIA. https://en.wikipedia. org/wiki/Transmetro politan. make it possible to colonise the Moon by producing food and other materials by directly assembling molecules.

Also in Peter F. Hamilton's Great North Road (Hamilton 2012), 3D printers manufacture a range of items from a default raw material. An expedition to an alien planet fabricates everything from parkas to modular housing.

The Cornucopia plot is not, however, always entirely optimistic. As already suggested by the replicator narrative in Star Trek, there are also some risks entailed by the cornucopia. One of them is the impact that abundance can have on human society. Singularity Sky (Stross 2003) is a novel by Charles Stross, published in 2003. The novel tells how human civilisation - after an epochal crisis, which destroyed most human settlements on the Earth -regains higher technological levels thanks to the "cornucopia machines", molecular assemblers that can recreate objects in predefined patterns or duplicate others. Some groups refuse, however, to use these molecular assemblers in order to protect their social order. When - notwithstanding the opposition of conservative groups - the cornucopia machines are finally made available to everybody, they cause the collapse of the existing social, economic and political orders. "People suddenly gifted with infinite wealth and knowledge rapidly learned that they didn't need a government". The cornucopia machines undermine the social order by weakening the principle of authority and reality, and creating a condition of anarchy ruled by an overarching principle of pleasure, dictating the immediate - almost hallucinatory - satisfaction of any desire.

\subsubsection{The Magic Bag}

In SF literature, 3D manufacturing is frequently associated to an endless production of goods (we have already met this aspect in the Cornucopia myth) and to unexpected tricks. Both themes recall a classic fairy tale trope, the Magic Bag. The Magic Bag - which could be a sack, a purse or a knapsack - is a typical fairy-tale theme, classified by the ATU system (Uther 2004) as type 569 (magic objects). Sometimes, the Magic Bag works as an object of concealment, used to hide the hero, or to hide something safely out of the sight (e.g., the magic bag in which to put Medusa's head, given to Perseus by the Hesperides in the Greek myth). In other cases, it provides an ever-empty supply of money, or other valuable items (e.g., Fortunate and His Pursue, an ancient European fairy tale, first transcribed in 1509, included in The Grey Fairy Book (Lang 1905) by Andrew Lang in 1905). There are many variants of this tale, yet the plot always includes two typical elements, i) the magic bag was given to the hero as a reward

\footnotetext{
97 https://en.wikipedia.org/wiki/Transmetropolitan

98 http:/ / boingboing.net/2015/05/22/the-man-who-sold-the-moon.html
} 
for kindness (e.g., Brothers Grimm's story "Brother Lustig" 99), ii) during the story, the bag is used for tricking the devil, the villain, or any evil monster (e.g., the Irish folktale "Billy Duff and the Devil"100). The latter feature is particularly interesting, because it often emerges from SF literature, and its presence almost unavoidably suggests the presence of a Magic Bag scheme in a given narrative.

For instance, in Dave Luckett's Patternmaker (Luckett 1994) 3D printers are used to make paradoxical shapes that first puzzle, and eventually discourage, extra-terrestrial invaders. Similarly, in Jennifer Foehner Wells' Confluence (Foehner Wells 2004), 3D printers are used to help the hero to figure out how to get the alien ship to do what he wants it to.

Sometimes, the Magic Box scheme involves that 3D printers are used for illegal purposes. Many stories are about 3D printers used for printing illegal drugs. For instance, this idea is in the plot of William Gibson's The Peripheral (Gibson 2014), which is about the story of Flynne Fisher, who makes her live by assembling product at the local 3D print shop. Similarly, in Cory Doctorow's short novel Printcrime ${ }^{101}$, 3D printers are used to reproduce almost everything, included illegal drugs. Two original variants of the magic bag doing illegal tricks are included in the video game Fallout and in Charles Stross' novel Rule 34. In the video game Fallout - based on a post-apocalyptic setting, in the aftermath of a global nuclear war - the player can use a molecular assembler to transform casino chips into several different useful items. Charles Stross' Rule 34 (Stross 2011) (Error! Reference source not found.) includes a section about a low-grade criminal who prints illegal products from an illegally-modified 3D printer, where the modification allows it to make contraband items like gun parts. His 3D printer

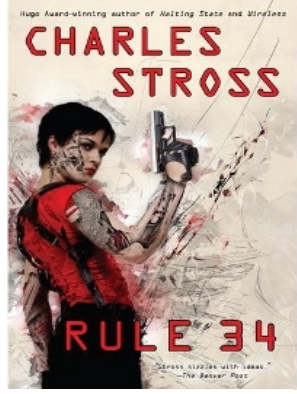

Figure 14 Rule 34. Source: WIKIPEDIA. https://en.wikipe dia.org/wiki/Rule _34_(novel). contracts a virus and uses up his feedstock while cranking out unlikelyproportioned sex toys.

"Illegal tricks" comprised in the Magic Bag scheme are rarely dramatic or catastrophic criminal behaviours, more often they are petty crimes, sometimes even justified by the context or by the plot. Basically, the Magic Bag scheme entails a rather optimistic perception of 3D printing technologies, which are not seen as an epochal technology, about to overturn all human standards. 3D manufacturing is rather described as a nice technological innovation, possibly having significant effects on human labour and manufacturing, but without major societal impacts.

\subsection{Disc ussion}

We have extracted three main archetypal stories from SF literature on 3D printers, 1) the Pandora's Box, 2) the Cornucopia, and 3) the Magic Bag. These three plots could be considered the "foundational myths" of 3D manufacturing in the collective imaginary, at least as it is captured and portrayed by science fiction.

The significance of these fantasies in terms of public acceptance of 3D technology is self-evident, there are a few points that deserve to be briefly discussed.

\subsubsection{The Trojan Horse Effect}

The "Trojan Horse Effect" is the peculiar situation in which an apparently beneficial item is welcomed, being unaware that it hides a mortal danger. When the Trojan Horse Effect is only imagined, it is a paranoiac experience, cantered on the anticipation of the disastrous result of an expected gain; or on the

\footnotetext{
99 Brothers Grimm (1815). Brother Lustig, Grimm's Fairy Tales, http://www.grimmstories.com/en/grimm_fairy-tales/index?page=1

100 Emerson P.H. (1984). Billy Duff and the Devil. In "Welsh Fairy Tales and Other Stories", http://www.gutenberg.org/ebooks/8675

101 Doctorow C (2006). Printcrime. Nature 439, 242 http://craphound.com/stories/2006/01/12/printcrime/
} 
(mis)perception that a dangerous agent is masked within an innocent garb. Quite often this effect becomes a component of self-fulfilling prophesies, because it unavoidably creates a social environment permeated with suspicions and mistrust. The "Trojan Horse Effect" plays an important role in the Pandora's Box type narratives, and it can be also present in the Cornucopia and in the Magic Bag schemes. This should warn against the risk the 3D printing technology could be perceived, soon or later, with some significant ambivalence. As it stands, there is no evidence of conspiracy theories including 3D manufacturing in their plot. Yet, overemphasising its positive aspects and its benefits could provoke the opposite result to excite techno-paranoia. If one wants to avoid such a risk, one should handle with care the positive messages. It is paramount to avoid giving the impression that any side effect of, or risk entailed by, this technology is purposely ignored or underestimated. A careful risk assessment must become integral to any technology system based on 3D printing, and 3D printing technology deployment should be always based on a strict application of the precautionary principle. Notably, those applications which could have disruptive effects on the labour market must be wisely monitored and their societal impact should be assessed in advance, recalling that one of the main SF narratives on 3D printing describes this technology as responsible for the collapse of an entire social system.

\subsubsection{Bodily Metaphors}

There are many clues suggesting that, at a deeper psychological level, imaginaries surrounding 3D printing are based, or originate, on metaphors concerning the body. As computers are often been conceptualised in terms of mental metaphors, and the human mind has been interpreted by using computer metaphors, similarly 3D printing is connected to metaphors concerning the body and its generative capacities. In particular, both the Pandora's box and the Cornucopia myths hint at a strict association between 3D printing and the maternal body. Imaginaries based on the Pandora's box plot unavoidably evoke fantasies about the maternal womb, imagined as a marvellous, but also aweinspiring, source of creation, an Alchemist's forge (Eliade 1956), where life and death inextricably overlap and mix. Likewise, narratives based on the Cornucopia scheme suggest fantasies about inexhaustible maternal breasts. They also recall, however, that over-gratifying experiences entail some risks of addiction and even obnubilation. Finally, also the Magic Bag plot might evoke bodily associations, notably associations with the digestive tract, stomach and bowel. It is a container which could hide things, making them to disappear, as the stomach does. In the while, it is a never empty container, which produces a stream of money, as the bowel produces faces (and at a deeper psychological level, we know that there is an equivalence between faces and money).

The underlying presence of these significant bodily fantasies should invite to be particularly cautious with all 3D printing applications entailing the production of human tissues, or body parts, or aiming at modifying the body structure. It is difficult to predict the way in which the public could perceive technological applications addressing the human body and it would be necessary to carry out further, and more systematic, studies on likely psychological impacts of 3D bio-printing before implementing these technologies at large scale.

\subsubsection{The Uncanny}

Both the "Trojan Horse Effect" and bodily fantasies share an element, which is the "uncanny". The presence of a feeling on "uncanny" associated to some technological applications is well known, and it has been first studied in robotics. In robotics, we call "uncanny valley" (Mori 2005) the sudden fall in affinity and sympathy for robotic artefacts when they become too like humans. Usually, a human observer's feeling of affinity for the robot increases as the robot's human likeness increases, till a given point, beyond which the observer shows uneasiness and discomfort, even horror. The analysis of the collective imaginaries surrounding 3D printing, that we have carried out through SF narratives, suggests that any form of "uncanny valley" could also occur in the future with 3D manufacturing.

The likely presence of an uncanny effect associated with 3D printing - although it is still embryonic should be taken very seriously, suggesting studying very carefully the best design strategies to increase 
users' acceptance. Technology design is never a trivial issue, and, in the case of 3D printing, it is likely to play a pivotal and critical role in the future. 


\section{STEEPED Aspects}

In this section, we review ethical, social economic, demographic and environmental aspects of bio-AM.

\subsection{Ethical aspects}

\subsubsection{Safety}

3D printing will make it possible to manufacture devices and products in a decentralised way. Since our current product safety regulations depend on centralised manufacturing (safety testing and regular inspection in factories), 3D printing raises questions about safety issues (Neely 2016). A shift of regulation from the product to the process/software level will not solve the problem because it is difficult to restrict the illegal distribution of files. Neely argues that creating an unsafe design is not, in and of itself, unethical, but distributing an unsafe design raises ethical issues (Neely 2016). One critical question is whether users can judge the safety of products by themselves, a requirement that was so far not needed with centralised production. At the moment, much of 3D printing is experimental in nature, and the communities of users probably realise that there is a degree of risk inherent in trying out various designs. In the future, it will be less likely that consent to accepting safety risks is given, in fact, informed. In the medical sector, not only CAD files and machines plus a standard material make the printing product, but living material has to be prepared (at the place of production) or chemical substances of high quality used. According to Heinz Redl (Ludwig Boltzmann Institute of Experimental and Clinical Traumatology, Vienna), a tissue regeneration centre as part of a hospital might arrive at some point, but only for large University Centres. Redl does not believe that such infrastructures will make their way to trauma surgery clinics. Therefore, major benefits of 3D printing might be a truly personalised medicine as well as increased availability (compared to donated material), rather than distributed production. Though the principle of quality controls in certified production centres may thus not be challenged in this application area, safety regulation has to keep pace with technical developments in order to ensure the safety of products.

Besides the more far-reaching perspective of safety issues connected with the potential rise of consumerproducer ("prosumer") additive (home) bio-manufacturing, worker safety issues are already being discussed with respect to 3D printing. While health risks such as oil mist and noise can be avoided with 3D printing, AM processes may create new health problems (Huang et al. 2013): the health risks of some of the materials used in AM are not yet well understood. Also, cells and living tissues should be handled under strict safety procedures as in laboratories, to prevent distribution of e.g. blood-borne diseases when patient material is used in manufacturing.

\subsubsection{Security}

There are also potential security issues, not only on an individual but also on a collective level. Dual use of 3D printing technology, e.g. in the case dangerous viruses will be printed (Neely 2016), is discussed in the media. However, we have to remember that this danger is not specifically linked to 3D (DNA) printing, but it is also possible with already existing genetic engineering methods.

\subsubsection{Data and privacy}

The question of protecting ownership rights and privacy emerged as important through all activities of the project. Ownership of medical data and bodily material was initially closely connected to issues of intellectual property rights. From expert interviews and literature it is clear that the development of 3D printing and related developments as 'do it yourself' (DIY) communities and data sharing puts current practices of intellectual property rights (IPR) under pressure.

The issue of collecting data emerged also in the interviews with experts. Michael Hasenpusch (research and development unit of Ottobock, Germany) states that Ottobock does not collect data in an international database as the uncertainty regarding data distribution is too high. To date, processes such as technical 
services are not digitalised and connected, although Ottobock's smart products are collecting data continuously. These data are only locally read for servicing the prostheses. Peter Dubruel (Ghent University) and his group declared that they were not involved in the gathering and handling of patientspecific data at the moment. However, they might be with the ongoing research activities on the meniscus implant. They require the local government to implement adequate rules as, for example, to make the medical scans impersonalised.

The need for ensuring privacy has been discussed also in relation to the innovation policy, which can be developed in the direction of implementing the patenting system or, at the opposite, granting open source culture. For example, the interviewed expert Michael Hasenpusch (research and development unit of Ottobock, Germany): states that Ottobock engages in a project of the Association of German Engineers (VDI) on data management and security in the digital workflow. Dr. Hasenpusch sees challenges in the processing, utilisation and analysis of big data due to restrictions because of data sensitivity and processing capabilities.

Øyvind Halaas (Professor in Nanomedicine, Department of Cancer Research and Molecular Medicine, Faculty of Medicine and Health Sciences, St. Olavs Hospital, Norwegian University of Science and Technology) is concerned about liability and patent issues. He claims that a national core facility sponsored by the state that takes care of liability until a certain technology is developed would be a good idea. Peter Dubruel (Ghent University) and his research group, which launched a start-up providing research equipment for scaffold fabrication marketed in the UK, see especially small start-ups suffering from the easy spreading of data.

\subsubsection{Reduction or increase of animal experiments?}

Another topic of ethical relevance is the hope, and claim, that bio-AM may contribute to a reduction of animal experiments, e.g. by fabricating physiologically functional tissues in limited sizes to be used for medical research and toxicology assays for cosmetics (especially relevant since the EU ban on cosmetic testing ${ }^{102}$ ) instead of animal models. Organovo is the first commercial company in this field with a patented platform (Organovo's NovoGen Bioprinter ${ }^{\circledR}$ Platform ${ }^{103}$ ).

However, further development of bioprinting may (at least temporarily) still rely on animal experiments and the use of animal material. Reasons are both the scientific experimental culture in life sciences (which is framed around the centrality and inevitability of animal experiments) and the current regulations about clinical trials (which render animal experiments a de facto obligatory step before human clinical trials due to ethical reasons). Furthermore, in specific applications of tissue engineering, such as aortic valves, cells from pigs are used in humans (which is then called xenotransplantation).

\subsubsection{Issues around organ donation}

A multitude of ethical issues surrounding organ donation would be solved entirely in a possible future in which organs are 3D printed. There would be no decision dilemmas regarding organ allocation (Ahearn 2016), and no scandals (Dufner \& Harris 2015) due to non-transparent organ distribution by hospitals any more. The highly contested field of xenotransplantation (Haddow et al. 2010; Cook 2014; Sanner 2001), which tries to engineer animal organs for transplantation into humans, would become obsolete. Psychological problems of patients receiving e.g. a heart from a deceased person would not be an issue any more (Bunzel et al. 1992). Human organ smuggling would become less economically rewarding (Scheper-Hughes 2000, 2003).

In a more realistic setting, due to limited production capacity and high costs there may be waiting lists for printed organs as well. An ethically relevant negative outcome of the feasibility of organ printing may be that the motivation to donate organs would probably decline and lead to an overall worse

102 https://echa.europa.eu/documents/10162/13628/reach_cosmetics_factsheet_en.pdf

103 http://organovo.com/science-technology/bioprinted-human-tissue/bioprinting-advantages/ 
situation for people in need of an organ. Only those that can afford to pay for their own organs might benefit then (Vermeulen et al. 2017). Furthermore, first clinical tests on humans can be expected to bear risks different from traditional organ transplantation for the patients, e.g. due to the possibility of cancer development (Vermeulen et al. 2017). Bioprinting solid organs is a long-term prospect, so it is also an ethical obligation to avoid hype and raise expectations that cannot be met in the near future (Murphy \& Atala 2014; Mandrycky et al. 2016). A very controversial issue that needs to be addressed before technical development gets so far is whether bioprinted organs will or should be patentable, and what this would mean for the autonomy of patients (Vermeulen et al. 2017).

\subsection{Social aspects}

\subsubsection{Social justice and accessibility}

Social justice and issues of access in particular are important social aspects of bio-AM. Enthusiastic statements concerning potential benefits are widespread, such as the claim that the technology will significantly "reduce health inequalities worldwide because medicine becomes accessible for everyone" and enable "monumental improvement for developing countries" 104 . While such generalisations are questionable, there clearly are potentials for, and some evidence of, AM technology improving the health situation in developing countries. Current benefits are obvious in the field of prosthetics and orthotics, in which AM technology is widely seen as advantageous, since it enables the on-site production of low-cost and highly individualised prostheses. The Victoria Hand Project (VHP), as an example, has the mission to provide upper-limb prostheses to amputees in need, with a focus on countries where amputees have limited or no access to prosthetic care. ${ }^{105}$

\subsubsection{Job creation or job losses}

Another important social aspect is the question of whether and to what extent the rise of AM technology in the medical and healthcare sector could cause the loss of jobs. AM technology could, for example, put pressure on the job market prospects of dental lab technicians. This was also an issue of concern expressed at the previously quoted workshop on "The 3D Printing Revolution and the Dental Sector: Opportunities and Challenges", organized by the EFDD group of the European Parliament on 30 November 2016. At the same event, representatives of dentists also warned against the dentists being somehow bypassed or losing importance as a consequence of technological progress. They insisted on the changes to be guided by prioritising patient wellbeing, not profit, warning against deregulation in this area. However, Øyvind Halaas (Professor in Nanomedicine, Department of Cancer Research and Molecular Medicine, Faculty of Medicine and Health Sciences, St. Olavs Hospital, Norwegian University of Science and Technology), who works on soft implants, thinks that AM will not compete with traditional fabrication methods but merely fill a gap of fabricating products that could not be done before. This will create jobs rather than destroying them due to automation.

A study on AM for the European Foundation for the Improvement of Living and Working (Eurofound) argues that changes in social tasks will become more important due to AM (Van Berneveld and Jansson 2017): Teamwork is no longer confined to individual steps of the production process, but also concerns closely linked steps such as design, material and process engineering, process operations and postprocessing. Moreover, the authors state that collaboration with external designers and material engineers and providers is expected to increase and argue that multi-disciplinarity becomes more important. These are also issues of concern for labour associations such as those in the European Trade Union Confederation (ETUC). Studies on the impact of AM on working conditions are scarce, a situation that trade unions find important to change. One of the concerns is that an increase of small-scale production locations may lead to less well-regulated labour conditions, for example with respect to

104 https:// www.raconteur.net/healthcare/how-3d-printing-could-revolutionise-medicine, updated on 8/30/2016

105 https:/ / www.victoriahandproject.com/ 
working times and wages. One considerable social aspect related to the labour market, is that the degree of organisation of workers may decrease, as maker spaces and home 3D printing become more prominent.

\subsubsection{Hype}

An issue that arose in the envisioning workshop and was further mentioned in the interviews is hype and too high expectations in the fruits of 3D printing.

Eduard Arzt (CEO and Scientific Director, Leibniz Institute for New Materials, Saarland University, Saarbrücken, Germany) and his group use two-photon lithography, a high resolution lithography-based AM technology for manufacturing grasping devices that are based on the gecko effect. Drawing from observations from scientific conferences, Prof. Arzt believes that the technology is still hyped and that it will probably not be able to keep the credited promises. Referring to the general phases of technology development, Prof. Arzt believes that a phase of disillusion will follow, where people will realise that certain credited possibilities cannot be fulfilled. After this phase, AM will be integrated in the daily life, people will have a better understanding of the process and be aware of its capabilities, advantages and drawbacks. Øyvind Halaas (Professor in Nanomedicine, Department of Cancer Research and Molecular Medicine, Faculty of Medicine and Health Sciences, St. Olavs Hospital, Norwegian University of Science and Technology) thinks that hype around AM has already faded, especially in bioprinting. Halaas specifically mentions one research endeavour, where people printed a scaffold for the tissue engineering of an artificial ear grown on the back of a mouse. This was not a functional ear but just a shape and the trust in the technology has since faded after this trigger of public attention.

According to a recent analyses by Gartner 106 concerning the hype cycle for 3D printing, which tracks media mentions and marketing efforts, 3D bioprinted organ transplants and drugs are on the rise, 3D printed surgical implants already reached the hype peak, and medical devices and human tissues are sliding into the through. 3D printed dental devices are climbing the slope and hearing devices have finally entered the plateau.

\subsubsection{Blurring the boundaries between therapy and enhancement}

One of the main topics of the project was the exploration of possible human enhancement applications of bio-AM: since the horizon scanning, it was noticed that in some cases human enhancement applications are pure promises or hype; in other cases there is a general uncertainty whether some applications are actually therapy or a means for enhancement.

The link to the blurred boundaries between therapy and enhancement and the risk to rely upon technological fixes to solve psychological or social problems were highlighted also in the interviews. Thomas Bayer (dental patient) reports from his experiences during the treatment of his congenital condition (that leads to teeth deterioration) that one of the first patients at the clinic was a professional model wishing to have "unnaturally" white teeth, and she was given an appropriate set. This threw up the question of cosmetic replacement and, if possible, reversibility. For theatre use, the latter is of course decisive as the gluing process is polluting and unhealthy. 3D imaging and, ultimately, AM production may offer entirely new possibilities not only for professional use but also for fashion. As alterations of the teeth have a long tradition (e.g. inserting jewels or grinding), altered replacements printed individually to personal taste, colour and shape may offer a new business model. Vladimir Mironov (3D Bioprinting Solutions, Moscow) said that technology dictates technological development. People of today are a slave of technology that continuously changes and adapts. Combining humans and robots with elements of AI, big data processing and analysis capabilities could be beneficial for humans because it could dramatically augment human capabilities. Similarly, Øyvind Halaas (Professor in Nanomedicine, Department of Cancer Research and Molecular Medicine, Faculty of Medicine and Health Sciences, St. Olavs

106 See, for example, https:/ /3dprintingindustry.com/news/gartner-releases-2017-3d-printing-hype-cycle$118349 /$. 
Hospital, Norwegian University of Science and Technology) thinks that with regard to human enhancement, AM will maybe help to improve senses or body performance. AM might provide complex implants integrating tailor-made and moving parts. AM will also likely play a role in cosmetics and in the military. Halaas believes that a lot of people would appreciate to be worked on by 3D printers. The military would also appreciate potential enhancements possible through AM. Halaas believes that AM will be everywhere in the future as an integral part of society.

Although there are possible concrete issues of enhancement, especially in cosmetic applications, technology assessment work and experts pointed out that there is a need to check the reality of promises and expectations of these emerging technologies. To avoid hype has been described as crucial in order to focus on an appropriate policy on bio-AM. The answer to this question is again crucial for economic reasons, as reimbursement through health insurances will certainly not apply, and thus products will be less easily sold, for non-therapeutic applications. Applications with aesthetic aims such as teeth or skin or facial tissue printing could be relevant in this respect; social and ethical aspects of just access are also linked to this regulatory issue.

\subsection{Economic aspects}

AM gets ever more important in economic terms. The worldwide revenue from AM consisting of all AM applications (products and services), not only of bio-AM, is estimated at $\$ 5.165$ billion (4.733 billion euro) and grew by $25.9 \%$ in $2015^{107}$. This estimation does not include research and technology development, or revenues from AM manufactured parts at the OEM (Original Equipment Manufacturer), e.g. Lima Corporate's acetabular orthopaedic implants. In a 2014 survey of more than 100 manufacturing companies, it was stated that $11 \%$ had already switched to volume production of 3D printed parts or products. According to Gartner analysts, a technology is "mainstream" when it reaches an adoption level of 20\% (d'Aveni 2015). In 2014, sales of industrial-grade 3D printers in the United States were already one-third the volume of industrial automation and robotic sales. Some projections have that figure rising to $42 \%$ by 2020 ( $d$ 'Aveni 2015). The biggest industrial sectors are industrial/business machines (20\%), aerospace (16.5\%), consumer/electronics (13.1\%), medical/dental $(12.2 \%)$ and academic institutions (10.5\%) (Wohlers et al. 2016) ${ }^{108}$. Wohler's report also shows the worldwide distribution of AM applications. Not surprisingly, North America harbours almost $40 \%$ of all installations of AM systems in the world, followed by Europe and Asia and the Pacific Rim by an almost equal share of about $28 \%$. In Europe, it is Germany, and in Asia, it is Japan that is best equipped, with $8.6 \%$ and $9.4 \%$, respectively. Over time, after having suffered a short decline, AM seemed to have experienced a real hype from 2008 on. This is mirrored in the development of CAD installations that more than doubled from 2009 to 2015, and the revenues from 3D printing, which soared from about $\$ 1.000$ (€916) to more than $\$ 5.000$ (€4.582) per unit during the same time span. While the selling price per system, originally about $\$ 118$ (€108), dropped by almost half over the 2000s, it rose again after 2010 . In particular, desktop systems became popular, their sales rising from a handful in 2007 to more than 278,000 in 2015.

This enormous development and the growing importance of AM as a way of fabricating innovative products has also had an impact on the medical and dental fields. Undoubtedly, they play an important role in AM, with almost one eighth of all applications. A variety of commercial dentistry products are available, offered by different companies. In the field of hearing aids, D'Aveni reported in 2015 that the US hearing aid industry converted to 100\% AM in less than 500 days, according to one industry CEO, and that not one company that stuck to traditional manufacturing methods survived. 109

107 https://www.forbes.com/sites/tjmccue/2016/04/25/wohlers-report-2016-3d-printer-industry-surpassed5-1-billion/\#79e5439519a0

108 For even more recent statistics, see http://wohlersassociates.com/blog/2018/03/wohlers-report-2018/.

${ }^{109}$ However, it has to be taken into consideration that this branch of industry is already highly monopolised. 
Due to the increase of elderly in the population of many countries, it is argued that it is one of the most important challenges in the 21st century to deliver high quality, economically efficient healthcare to improve the average well-being of the population. Personalised medicine is seen as a promising area, because it tailors specific characteristics and needs of patients. The potential of 3D printing and manufacturing is seen as crucial in achieving this goal (Ely 2009). In 2006, the CUSTOM-FIT European initiative aimed at creating a fully integrated system for the design, production and supply of individualised medical and consumer good products (Gerrits et al. 2006).

Regarding the purpose of the medical objects fabricated, functional parts take the lion's share of almost one third (32.5\%). Fit and assembly parts make up $16.2 \%$, objects for education and research about $10 \%$. However, this field is particular as there are many rules for different applications such as implants or prostheses, surgical aids or dentistry. These regulatory requirements may give rise to economic hurdles, too, for the developing companies. Fears of novel technologies for the enhancement of various aspects of human performance above the normal may either present new market opportunities or trigger rejection among a reluctant public. (Norman et al. 2017) argue that the recent approval of a 3D printed drug product by the FDA will stimulate continual innovation in pharmaceutical manufacturing technology. The field of 3D printing of tissues and organs is also seen as important for testing drugs: There is potentially a huge market for the production of physiologically functional tissues in limited sizes to be used for toxicology assays, as it could save billions of dollars and euros for the pharmaceutical industry due to the high cost of animal experiments. The need for surgical implants has constantly increased in recent time, and the potential of 3D manufacturing is widely deemed enormous.

As variously mentioned above, AM provides considerable freedom in designing, manufacturing and using prosthetic and orthotic devices, where customisation is the key for products, relying on medical imaging or 3D scanning data. This enables quick reprints and changes in the designs, which could bring savings. ${ }^{110}$ Problems may be entailed as development and manufacturing times are long and associated costs are high. ${ }^{111}$ According to Michael Hasenpusch (research and development unit of Ottobock, Germany), AM does not make economic sense for most industrial applications yet, but machine and material prices will lower significantly in the upcoming years. Dr. Hasenpusch mentions that industries where AM already plays a big role can calculate the amortisation differently than a company like Ottobock might be able to, making AM relatively more economic for their use. Airbus, for example, can significantly reduce weight with brackets that are installed 1000 times in a single airplane, which subsequently provides savings in airplane operation costs. This opportunity does not exist for Ottobock. The company depends on private payers and individual reimbursements from insurances. Thus, the implementation of innovative prostheses may be highly dependent on reimbursement regulations.

Especially when combining the AM process with living biological material, overoptimistic predictions and poorly managed expectations may lead to, on the one hand, potential stakeholders avoiding AM because they are sceptical about its capabilities. On the other hand, early adopters might make false investments as they overestimate $\mathrm{AM}^{\prime}$ 's potential. It is foreseeable that the intelligent combination of different processes and technologies will bring the greatest benefits. In the past, hybrid systems often have opened new ways towards innovative applications for industrial production. ${ }^{112}$. This also applies to $\mathrm{AM}$ and its combination with other manufacturing technologies in the medical field. Highly personalised one-off products are needed in the medical sector, and this suggests that the pattern of

\footnotetext{
$110 \mathrm{http}: / /$ advancedmanufacturing.org/3d-printing-orthotic-prosthetic-devices/

111 Interview with Constantinos Mavroidis, director, Biomedical Mechatronics Laboratory, Northeastern University Boston.

112 http:/ / www.a3dm-magazine.fr/la-fabrication-hybride-au-dela-de-la-fabrication-additive/by Giorgio Magistrelli
} 
industrial emergence, technology adoption and diffusion follows the niche development and specialisation that has been observed in previous emerging industries. ${ }^{113}$

The market is getting ready for AM-related products, however, at least in the United States, where regulatory approval has been granted to several AM applications. Currently, over 20 medical implant products of AM technology have gained clearance from the FDA) (Wohlers et al. 2016). European regulation is under discussion, too, also against the backdrop of a future mass customisation enabled by bio-AM (Li and Faulkner 2017). There has been a recent revision of the Directives for Medical Devices and In Vitro Diagnostics. Also important in this context are the Directive 2004/23/EC on on setting standards of quality and safety for the donation, procurement, testing, processing, preservation, storage and distribution of human tissues and cells and the Regulation 1394/2007 on Advanced Therapy Medicinal Products (ATMP). Regulatory measures in these areas will make an impact on policies for the use of substances of human origin, such as human tissues and cells, and blood and organs. As also discussed below, the combination of materials (devices) and human biological material, in combination with alternative manufacturing processes for AM, have recently spurred debate on appropriate regulatory pathways in the EU. Currently, approval routes within the EU and across the world diverge, which may create inequality in access to therapies and services. Another regulatory issue which touches upon economic aspects of AM technologies concerns intellectual property rights, and whether newly constructed applications are, or should be, subject to open innovation principles. Related to this concern is whether decentralising manufacturing will lead to more private endeavours of R\&D and a balance of knowledge sharing and production between public and private sectors..

\subsection{Demographic aspects}

Some of the economic, ethical and social aspects mentioned in preceding sections have the potential to become also relevant with regard to demographic aspects. However, given the usually long-term and multifactorial character of demographic impacts and the relative novelty of bio-AM, any discussion about the latter's demographic impacts at this stage would be premature and entirely take place in the realm of mere speculation.

\subsection{Environmental aspects}

There is widespread consensus that AM has significant potential for an ecologically more sustainable economy. As (Huang et al. 2013) argue, AM is more efficient when compared to conventional machining processes in terms of virginal material consumption and water usage; it does not require the use of coolant and other auxiliary process inputs and thus produces less pollution to the terrestrial, aquatic and atmospheric systems and requires less landfill. Moreover, it may enable simplified supply chains and boost on-demand manufacturing, thus reducing the need for warehousing, transportation and packaging.

However, while there are already a number of contributions to life-cycle analysis (LCA) concerning AM's environmental impacts - some of which largely corroborate the optimistic forecasts (e.g. (Kreiger \& Pearce 2013) -, the flipside should not be ignored. As (Huang et al. 2013) point out, AM might not have an edge over traditional manufacturing processes in terms of overall energy consumption, once the entire operating procedure is considered.

Great expectations also become visible on a more general level, for example with regard to the potential contributions of AM technology to a circular economy (Despeisse et al. 2017). The point of view is widespread that AM holds the potential to enable the shift towards such a sustainable economy. While proposals have already been made on how to accurately and in detail assess the environmental impacts of 3D printed products at the design stage, there is obviously a need for further research on this topic. In an alternative scenario, it might bring about increased resource consumption due to less eco-efficient

113 http:/ / www.personalizedmedicinecoalition.org/Userfiles/PMC-Corporate/file/The-PersonalizedMedicine-Report1.pdf 
localised production, demands for customised goods and a higher rate of product obsolescence. Moreover, when items are cheaper and more easily available through AM, overall consumption might rise.

Apart from general issues of sustainability and resource consumption, environmental aspects have to be assessed depending on the specific material used for AM. In bio-AM, genetically modified or contagious cell material (infected with viral or bacterial diseases) could be involved that would need special care in order not to be released to the environment. 


\section{Foc us on three application areas}

To support further analysis, we reviewed three application areas in greater depth. These are orthotics and prosthetics, dentistry, and tissue and organ printing.

\subsection{Orthotics and prosthetics}

While orthoses are used to enhance or to correct sound body parts, prostheses are devices to replace lost or malfunctioning parts of the body. In general, the fabrication of orthoses and prostheses (O\&Ps) by additive manufacturing offers huge potential for several applications. The devices can be built as a whole, integrating joints, moving parts or sensors and actuators. Furthermore, they are more convenient to wear and more aesthetic, since AM allows to reduce weight, implement material property gradients (stiffness, elasticity, hardness, etc.) and integrate functional parts such as sensors and actuators. ${ }^{114115} \mathrm{In}$ addition, AM offers a high degree of design freedom and allows combining different materials in one build. In this regard, the design freedom in terms of internal structures and the combination of materials allow to fabricate biomimetic structures such as grasping devices leveraging surface effects (Arzt et al. 2003; Autumn 2006). In the field of orthotics and prosthetics, there are several application areas for AM.

One important application is the replacement of lost limbs. When designing a prosthesis, the healthy counterpart is often used as a laser-scanned model, so that the device is made as a mirrored replica of the healthy body part (limb, hand, part of the skull etc.) (Jin et al. 2015; Telfer et al. 2014). To design a custom orthosis or prosthesis, the shape of the device is fitted to the surface profile of a body scan (e.g. 3D laser scan). Here, fitting a prosthesis shaft to the residual limb is not only very important for the customer's comfort but also for avoiding inflammation and false postures. AM has the potential to improve the fitting in a less labour intensive way and requiring less castings, compared to traditional fabrication methods. Similarly, digital imaging by 3D laser scanning and AM have also been used for creating prosthetic socket fits for amputees since the development of these techniques in the 1990s (Rogers et al. 1991; Rovick et al. 1992; Rovick et al. 1996; Rogers et al. 2009). However, sophisticated designs were discarded due to the high-cost and complicated design process (Lockhande \& Crawford 2001; Rogers et al. 2001). Low-cost AM processes were employed and AM equipment suppliers for socket fabrication emerged, such as Rapid Manufacturing Machine (Ng et al. 2002) and Squirt-Shape ${ }^{116}$. To the best of our knowledge, there is no service for AM custom sockets to date.

Other widespread and cost-effective devices that can be fabricated by AM are foot orthoses (Dombroski et al. 2014; Milusheva et al. 2007) as well as additive manufactured ankle foot orthoses (Faustini et al. 2008), or custom insoles which were already commercialised by SOLS117 in 2013.

Besides external prostheses, clinical studies of implanted AM prostheses, in particular hip, knee and spinal implants and the correction of maxillofacial (skull) defects are becoming increasingly popular (Wohlers et al. 2016; Li et al. 2011). Further custom titanium restorations were implanted restoring nerve and artery channels in patients' skulls (Li et al. 2013; Wang et al. 2012). Also in the area of additive manufactured implants, some products have already been approved and/or cleared.

\subsubsection{Potential paths of development}

During the interviews, different aspects of potential developments in orthotics and prosthetics were highlighted. Michael Hasenpusch (research and development unit of Ottobock, an internationally leading supplier of prostheses) thinks that the fabrication of metal parts by means of selective laser sintering (SLS)

114 All internet resources have been last accessed 19.02.2018.

115 http:/ / www.3ders.org/articles/20150623-german-giant-ottobock-teams-with-unyq-to-develop-stylish-3dprinted-prosthetics.html

116 http:/ / www.engineering.com/DesignerEdge/DesignerEdgeArticles/ArticleID/10255/The-Squirt-Shape3D-Printer--a-Moonshot-Project.aspx

117 http:/ / www.sols.com/ 
is a future option for Ottobock; however, the return on investment potential depends on the utilisation of the respective machine.

The use of AM in series production may be another option. However, this has not been examined yet, which is partly due to the lack of competence in AM design. The required mechanical compliance and ability to think differently when designing products are major issues that hamper the further industrial manifestation of AM. Using only existing designs might reduce the advantages of AM. The potential and benefit of AM in key manufacturing processes will materialise with the internalisation of design possibilities in terms of geometric and functional complexity following bionic principles. Ottobock is working on a method to achieve the necessary freedom of design. AM will not replace conventional technologies; however, it will permanently complement them.

To this date, the AM fabrication of end user products is very limited. Polich, a partner institution of Ottobock in Traunstein, Germany, produces hand joint prostheses employing AM. The company Jablouse produces cosmetic covers for lower extremity prostheses. Dr. Hasenpusch questions, however, whether AM will be capable of becoming standard in a field that is traditionally marked by craftsmanship.

In the envisioning workshop it was highlighted that although today AM only plays a minor role in the production of O\&Ps, with foot orthoses constituting the largest portion, in 5-10 years technological development will be advanced enough to compete with traditionally produced O\&Ps. With highly advanced AM technologies, O\&Ps may even contribute to human enhancement by e.g. providing products that combine different technologies such as sensors, connections to cell phones etc. and may increasingly be used in the gaming industry or for "body art" (e.g. for soldiers in war, QS movements etc.). In other words, bio-AM has large technical potential and promises a lot, but rather than focusing on what is technically feasible, the discussion in this group focused on non-technical aspects such as basic human rights and how to ensure that bio-AM contributes to an inclusive society rather than discrimination based on capabilities.

In the scenario on prosthetics (Arms and Legs for Solidarity), no new technological development is described, since this is a scenario where technological progress is assumed to be slow or not to exist at all.

\subsection{Dentistry}

Dental restauration is also a special form of prosthetics and maybe the most advanced one, because dental laboratories are separate from hospitals and are the early adopter of new technologies and materials. The workflow for producing dental devices also starts with the digitisation of the anatomical site of interest, followed by data processing by a variety of software tools and manufacturing. Even though there are approaches to improving direct imaging of the site of interest in the patient's oral cavity (Güth et al. 2013; Vecsei et al. 2016), the common approach is to scan a model from the oral cavity and fabricate a cast prepared by conventional impression methods that are later digitised. AM provides the option of automatically creating wax models, an approach believed to result in high production rates, easy quality control and high precision wax patterns as well as in reduced finishing work on the casts (Azari \& Nikzad 2009; Liu et al. 2006) However, an open issue concerns how the fabricated models compare with those obtained from conventional machining or manual processes (Munoz et al. 2017).

Despite AM's niche existence so far, this technology has high potential for dentistry and is becoming increasingly popular due to its potential to overcome the limitations of machining (Torabi et al. 2014). The biggest advantage is the accuracy of production: parts can be tailored to the patient's hard and soft tissue (Davis 2010; Webb 2000; Örtorp et al. 2011; Quante et al. 2008). However, when directly fabricating implants, the porosity of metal parts fabricated by SLS can reduce their durability (Traini et al. 2008). Furthermore, the number of available materials suitable for direct AM of restorations is still limited (van Noort 2012). Due to the high costs of metal and ceramic AM machines, indirect fabrication of restorations via intermediate wax models is therefore still dominating direct fabrication. The biggest 
obstacles discussed in the literature are the high costs of the most common metal and ceramic processes (SLS or SLM) for many dental laboratories, together with the necessary time and competence in 3D printing. Further key challenges for advances of AM in dentistry may be the training of dentists, together with further research (e.g. on biocompatibility) and legal questions (e.g. reference standards).

\subsubsection{Potential paths of development}

During the project, we interviewed Thomas Bayer who suffers from a congenital condition called Osteogenesis imperfecta which causes impaired bone formation during juvenile growth and subsequent skeletal deformations (impairing also the teeth). He was offered an entirely new treatment called "full mouth recovery" based on a digital reconstruction from photographic data, as one of the first patients ever at the Dental Department of the Medical University Vienna. For Bayer, the initial expectations fully materialised. Both functionality and aesthetics turned out to be satisfactory. It is foreseeable that this method will soon completely replace the old-fashioned replacement tooth-by-tooth. It also implies less $\mathrm{x}$-rays, which will only remain for root and bone diagnosis.

In the envisioning workshop, it was stated that additive manufacturing techniques for dentistry were already advanced and implemented in daily practice. Basically, digital modelling enables dentists to create precise 3D maps of the patient's oral cavity, which can be transferred to dental labs anywhere in the world and used in the production of wax patterns and subsequent moulding of dental implants. To be sure, digital modelling can be - and is in most contemporary cases - coupled with traditional moulding methods. This means that the current state of implementation of additive manufacturing in dentistry is in fact dominated by a technique (digital modelling or CAD) which is technically peripheral to $3 \mathrm{D}$ printing of physical materials (CAM), such as additively manufactured teeth. This is because at the current state of development, traditional moulding techniques, such as ceramics, are still superior to existing 3D printing techniques in terms of surface texture and durability. However, there is great potential in switching to additive manufacturing for moulding. This potential includes a more precise fit and - for implants made of expensive materials such as Zirconia - as much as $90 \%$ lower material consumption. With these potential benefits in sight, many public and private investors continue to promote the development of CAM techniques for dentistry. From a technical development perspective, additional investments may be needed to perfect scanning technology and CAM techniques using new materials and surfaces. However, when taking into account the overall development trend in the field of dentistry, it is to be expected that such techniques will become refined to compete with traditional moulding techniques within the next 5-10 years.

If it is allowed, uses of additive manufacturing in dentistry may conceivably go beyond traditional medical applications into cosmetic applications. It requires no great leap of the imagination to envision increased flexibility and lowered costs translating into replacement dentures in the colours of the user's favourite football team.

Compared to the often discussed scenario in which "everyone will have their own 3D printer", it seems highly unlikely that the costs of additive manufacturing machinery will fall to the point where each dentist will naturally have his or her own 3D printer on site. It seems more likely that the default development trend will be for centralised printing centres which will amass different types of CAM machinery and know-how to serve multiple customers with CAM needs. Some such centres may specialise in dentistry applications, but it is also likely that centres will serve multiple markets at once. Such centres will present disruptive competition for dental labs and, everything else being equal, will pressure dental labs to adopt CAM technology and possibly also to centralise. This process of structural change in the dentistry sector is not bound by geography. It is fully possible to imagine new value chains that have more to do with how value chains develop in the digital economy than with dentistry; i.e. with US-based digital service providers acting as hubs receiving digital scans from dentists and distributing them to outsourced CAM centres in China, India or elsewhere. As in many other cases of 
digital disruption, such development could provide cheaper services to European citizens, but at the same time destroy value and jobs in European dental labs.

In the scenario workshop, the following developments were anticipated: the possibility of experimenting with printing real teeth from stem cells; the possibility of transforming teeth into a piece of art with sensors in the teeth that are connected to a mobile device and using an app to choose a different piece of art on a tooth that was made of responsive materials; the use of bite marks as a new identification tool, in addition to iris scans; the construction of a database of thousands of patients, all with a perfect bite, to know exactly how to shape new teeth for an individual; the scans of the inside of the mouth made by robot doctors.

\subsection{Tissue and organ printing}

With the development of biomaterials for AM, the automated fabrication of scaffolds by fused deposition modelling (FDM), bioplotter ${ }^{118}$ oder bioscaffolder (Chen et al. 2011) with tuneable, reproducible and mathematically predictable physical properties has become possible. So far, cell-free scaffolds, fabricated ex-situ and transplanted after their fabrication, have shown clinical success for bone, osteochondral tissue repair, cartilage and skin (Melchels et al. 2012). Among the applications considered promising are the use of FDM to create polycaprolactone scaffolds for bone tissue engineering (Schantz et al. 2006) and other polycaprolactone and multi-material scaffolds for bone and cartilage tissue engineering (Rai et al. 2010; Ho et al. 2010).

Furthermore, the inkjet printer technology has been explored to create controlled volumes of biological systems in a layer-by-layer fashion that is sometimes referred to as organ printing (Boland et al. 2007). In this way, cells and material can be deposited simultaneously. The interest in this approach has led to the development of many robotic systems to create cell-laden constructs mimicking various tissues, which are progressively commercialised. So far these matrices proved successful for cell studies including the development of blood vessels (Skardal et al. 2010), nerves (Marga et al. 2012), skin (Koch et al. 2012) and bone (Fedorovich et al. 2011). However, the approach is considered insufficient to produce large-scale organs and is far from clinical trials (Mironov et al. 2003; Mota et al. 2015).

The main bottlenecks are the complication of mimicking the cellular organisation of natural tissues, the economically feasible upscaling to clinical applications and insufficient vascularisation of grafts (Melchels et al. 2012). Furthermore, anatomically correct models are difficult to fabricate, requiring 3D data files with clear information on soft tissue (Mota et al. 2015).

$\mathrm{AM}$ as a tool in tissue engineering seems promising in combination with other cell seeding processes. The prospect of digitally controlling distribution of material and cells in $3 \mathrm{D}$, with high geometric complexity and accuracy, has made this field a very active research area, although clinical applications may result difficult, also due to the limited availability of biomaterials and regulatory hurdles.

\subsubsection{Potential paths of development}

Potential paths of development in the field of organ printing and regenerative medicine were largely discussed during our interviews. Heinz Redl (Ludwig Boltzmann Institute for Experimental and Clinical Traumatology, Vienna) and his group investigate how photopolymerisation can be done in the presence of living cells and organisms to create a biologically dynamic and tunable environment. He believes that AM's strength lies mainly in research and prototyping as AM is not effective for high throughput. A way to get this technology to the end consumer market in Redl's field is the tailored fabrication of patient-specific implants and surgery instruments, if parts of bones or structures, surgical guides and aids have to be mimicked and adapted to the anatomical site of interest. This is also strongly connected to advances in imaging and data processing that have to provide these design possibilities. In his opinion, a tissue regeneration centre as part of a hospital might arrive at some point, but only for

118 https:/ / envisiontec.com/3d-printers/3d-bioplotter/ 
large University Centres. Redl does not believe that such infrastructures will make their way to trauma surgery clinics.

Øyvind Halaas (Professor in Nanomedicine, Department of Cancer Research and Molecular Medicine, Faculty of Medicine and Health Sciences, St. Olavs Hospital, Norwegian University of Science and Technology) thinks that there are many products/designs that cannot be made without any other technology and hence that AM will play a role in future final production for complex geometries. It will not be used for products that can be made by other means, there it will remain merely a prototyping tool. It can never be a really fast process, so it has to be a process for difficult parts. Halaas is convinced that AM can keep its credited promises. He points out the versatility of the technology to produce automotive parts and tissue engineering scaffolds likewise.

Several concrete applications in the area of tissue printing are mentioned by Peter Dubruel (Ghent University). His main interest is materials research. With the materials the group is developing, they are advancing the three following fields in tissue engineering where future developments may be anticipated: 1) orthopaedics: meniscus/soft implants with use of the bioplotter; 2) oculus applications: two-photon polymerisation (high-resolution stereolithography) shall be used to create an implant for age-related macular degeneration (blurred or no vision in the centre of the visual field); 3) reconstructive research: lipophilic materials for cosmetics and surgery that are safe and implantable.

Yu Shrike Zhang (Brigham and Women's Hospital, Harvard Medical School) believes that AM will become dominant in the field [of tissue engineering]. His group works on a new technology for the AM of a core-shell nozzle system. It shall combine physical and UV crosslinking in one system. This technology will therefore be a combination of inkjet- and extrusion-based systems and will be able to fabricate different biomaterials and bioinks at the same time. This shall lead to the better mimicking of biomedical environments and optimised architectures. A large pool of bionics will be commercially available. Bones and other hard biological materials, especially small-scale ones are more mature and will enter into clinics sooner. Soft tissues are more complicated and will require more time. The most immediate products that may come out of their research are novel materials, respectively novel hydrogel formulations that can have use in the medical industry, as well as new printing technologies. A tissue engineering product will take some time, and the competition is very high. In regard to organisation, he thinks that customised products of the future will likely be made in a bioengineering facility inside the hospital utilising the hospital's imaging capabilities.

The following two interviewees described further-reaching visions and already mentioned new AM or other 3D printing technologies.

Vladimir Mironov (3D Bioprinting Solutions, Moscow) is pioneering the creation of 3D bioprinters and various artificial tissues up to entire organs (including vascularized and functional mouse thyroid gland). He sees the rapid evolvement of 3D bioprinting and its realisation in the near future. For Mironov, AM is a key enabler for the creation of artificial tissues, but maybe not the only or best technological approach to it. Mironov's vision is to create a scaffold-free tissue construct without requiring 3D printed material as support for the cells, using 3D cell and tissue patterning via magnetic, acoustic waves and electric fields. New fields will emerge such as the bioprinting of emerging creatures. Referring to this topic, Mironov pointed out the recent publications of Rahsid Bashir (e.g. Raman et al. 2017), in which the authors fabricate biological actuators for controllable locomotion on 3D printed skeletons. This may lead to the development of biotoys and biorobots with AI.

Regarding organisational issues, Mironov thinks that the stability and safety of tissue engineering constructs for transportation hampers outsourcing the fabrication. Therefore, Mironov envisions every hospital to have a complex integral regenerative medicine laboratory at their availability in the future. Mironov sees the future revenue in the medical biofabrication and bioprinting equipment sold to hospitals and clinics or to the integrated regenerative medicine labs, respectively. This will be a huge opportunity for suppliers. Furthermore, Mironov sees a big business in medical data processing software 
and artificial intelligence (AI). This includes data generation, processing and conversion into 3D printable files. This software will have an impact on clinics and education. In terms of processing data for 3D printing, the company Autodesk is dominating and controlling the market, and the revenue of the company is increasing fast. Software for surgical in vivo 3D bioprinters with elements of $\mathrm{AI}$ is very promising for advancing healthcare and the digital revolution in surgery.

Øyvind Halaas (Professor in Nanomedicine, Department of Cancer Research and Molecular Medicine, Faculty of Medicine and Health Sciences, St. Olavs Hospital, Norwegian University of Science and Technology) says that in the next 30 years, it would be his dream to have an AM technology for the fabrication of precisely resolved, soft, transparent and cell-compatible materials that can be fabricated at high speed, in large volumes and out of multiple materials. For Halaas, AM will not compete with traditional fabrication methods, it will merely fill a gap of fabricating products that could not be produced before. This will create jobs rather than destroying them due to automation. But the consumers themselves will be more involved in making their own products at home, downloading data and fabricating products through AM.

In the envisioning workshop, it was stated that bio-AM within the field of tissue engineering was expected to undergo huge developments and the expectations of the field were high. It was said that tissue engineering was likely to become commercialised over a 10-year perspective.

However, while tissue engineering is already highly advanced, the potential of organ printing is more speculative. Functional organs to be integrated into the human body are not likely to occur within the first half of this century. Some of the most promising applications include customised implants such as stents, and the products are likely to become fully integrated with the human body and cells within the next decade. Mini organs are currently being bioprinted, and in some cases these are partially functional. Likewise, partial replacement of the liver and regeneration of skin are realistic achievements over the next decade, when skin and liver pieces may even be manufactured during an operation. Despite this, AM organs would first of all be useful for educational purposes to simulate surgical interventions, while more advanced organs, such as hollow organs, are unlikely to be manufactured within this time horizon. The advancements in these technologies are, however, hard to predict and could rapidly change, allowing for more advanced usage. The technology would be advanced at a level where clinical trials could be conducted on simple tissue. 


\section{6. $\quad 360^{\circ}$ Envisioning Workshop}

To develop a broader understanding of the wide range of potential implications of bio-AM and provide a clearer picture of the policy challenges relevant to MEPs, a 360 degree envisioning workshop was conducted focusing on three promising application areas for bio-AM: dentistry, prostheses and orthoses, and regenerative medicine and organ printing. This section summarises the process and key findings from the workshop including technological development trends, the role of regulation and building blocks for the scenarios.

\subsection{Methodology}

Highly supportive regulatory framework Extensive implementation

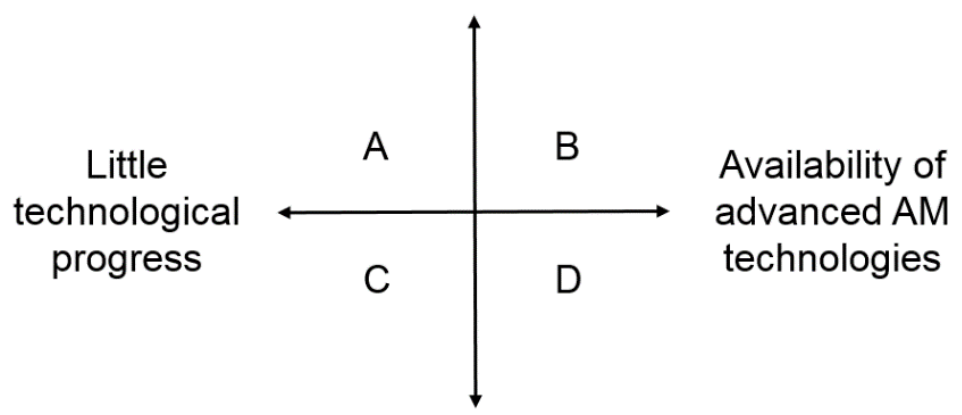

\section{Little regulatory support Low levels of implementation}

Figure 15 Framework for building the future scenarios by asking "what if..." for each quadrant (A, B, C, D).
The workshop was structured around the STEEPED approach where each of seven dimensions of potential impact was discussed in relation to the degree of technological development and the level of regulatory support. For each application area, four future scenarios were developed from a 5-30 year time perspective.

A total of 23 people participated in the workshop: one Member of the European Parliament, three STOA staff members, ten external experts/stakeholders, and eight project partners. The participants were divided into three groups, each group covering one application area. After a short general introduction to the previous findings of the horizon scanning, the participants initiated a brainstorming on their hopes, dreams and fears for the future in relation to the AM application area of their respective group.

The seven STEEPED dimensions were visible during the brainstorming at the tables and listed on easily readable A4 handouts along with the key results of the horizon scanning. The participants were asked to challenge the assumptions and brainstorm on any other possible impacts to occur in the future for this particular application area. After the initial brainstorming, each group developed four future scenarios from two parameters: (1) degree of technological advancement and (2) level of regulatory support. The groups were allowed to build the scenarios using different time perspectives. In a plenary discussion, two scenarios developed by each of the groups were presented, allowing the rest of the workshop participants to comment on the scenarios and provide their view. During the plenary discussion, key elements of the group discussions were brought up and compared to the other future scenarios.

\subsection{Findings}

In this section, we present the building blocks used as starting material for the writing of the first version of the techno-moral vignettes. They illustrate how the regulatory framework and the level of technological development may change the role of bio-AM in society in a ten-year perspective. A, B, C and $\mathrm{D}$ refer to the four quadrants in Figure 15. The building blocks already contain different policy options and possible outcomes for each of the three specific application areas of bio-AM. 


\subsubsection{Regenerative Medicine/Tissue and organ printing}

\section{A. Disappointment and frustration}

- Despite high investments and a highly supportive regulatory framework, technological promises and people's expectations of regenerative medicine and organ printing have not been fulfilled. The technology has shown to be insufficient in solving basic medical issues, and in Europe there are only few labs that use the technology. In other words, AM is still a niche technology, which again entails that AM is not developed further and gets stuck in this niche. It may be applicable in some rare instances, but the demand is low and a broader use is not foreseeable. Low applicability and demand entails a lack of infrastructure and development, which again makes AM not very attractive to spend any research effort on it.

- The innovation process is stuck in the early phases, disappoints and creates frustrations in society.

\section{B. Butchers showroom}

- Everyone with physical or health-related problems can technically find a solution to the problem. In Europe, everyone has access to a spare pair of organs and regulatory bodies worry about overuse. The technical possibilities stress the healthcare systems because the therapies are often expensive and are associated with regenerative problems. This leads to a paradigm shift in the healthcare system where focus is directed towards more preventive efforts rather than fixing problems. Meanwhile, we see an increase in medical tourism since regulation is not uniform globally. Along with the medical use, there is a growing demand for non-medical applications.

- The technological advancements will particularly be used for human enhancement treatments including aesthetic enhancement. The number of people choosing the "perfect" skin pigmentation and/or surface texture and other changes of the natural body look is growing and the expectations for further technological development are high.

- The increasing freedom to technically choose how the human body should look and function will create a gap between the natural humans and the enhanced. The regulatory framework focuses on overuse of the technology and how to dampen people's expectations for bio-AM to solve their problems.

\section{The blues}

- The scenario provides limited regulatory support and the implemented regulations create bureaucracy for start-ups rather than moving the technology forward. Professionals with the vision to develop the technology move to other countries where there is better regulatory support, making it irrelevant to put in place further regulations in Europe.

- The slow progress in the development of these technologies makes them useless and gives people a negative perception of them. The innovation communities are attracted to other technologies, which will develop at a faster pace. These other technologies will gradually integrate bio-AM, thereby building bridges between the innovation communities and further advancements in bioAM.

\section{Hit the road}

- The black market is dominating the market for bio-AM since the technologies are primarily available in the private sector outside Europe. This results in a growing medical tourism away from Europe to the rest of the world. 
- There are no standards for the quality and safety of the products and treatments and, as a matter of fact, people do not trust the providers of the services. People using the technology include rich people and the bio-hacker communities.

\subsubsection{Prostheses and orthoses}

\section{A. Technological progression with early adopters and open access}

- Additive manufacturing of O\&Ps is successful despite little technological progress, but it is still a niche that competes with traditionally produced O\&Ps. They serve as simple solutions to perfect fit, but are primarily produced in the health sector for medical treatment. Those who benefit are the researchers within the technical field who receive funding to develop the technologies as well as the early adopters including children and volunteers who are open minded to new technologies.

- In the high-income countries, the early adopters could be children with missing limbs, while early adopters in the low-income countries may be the poor, who are more likely to accept a product that is not fully functional. The market for medical recovery using AM O\&Ps is global, while the need for aesthetic enhancement using AM O\&Ps is higher in the high-income countries.

- Regulations support technological development by funding research and improving infrastructure for CAD. However, there is also political support towards civil society organisations (CSOs) working with issues related to amputees in order to go in the direction of a more inclusive society that brings down taboos of using prosthetics. This process is highly participatory, involving civil society and different stakeholder groups including the resource poor.

- Open access policies are implemented to improve the providers' access to CAD (including CSOs). The open access policy is particular beneficial for the low-income countries and for the small nonfunded players.

\section{B. Active consumerism}

- AM within the field of O\&P is extensively used to recover the millions of people in the high- as well as the lower-income countries with missing or defect limbs. The technology is easily accessible worldwide due to the low cost of the products and the open access to CAD.

- In the high-income countries, we see more technological progress compared to the low-income countries and the AM technologies are highly integrated with other technologies such as AI and ICT. This means that the products often provide better solutions than traditional O\&Ps, hereby expanding the market to a broader user community that not only includes children but also elderly, veterans, people with traumatic injuries and people with diabetes.

- The regulatory framework changes from technological support to non-technical support, hereby supporting human rights such as the human right to physical integrity. Other societal challenges arising from the technological development will also be addressed, such as issues of 'dual use' (dual use 'therapy and enhancement', including military uses), social acceptance of medical recovery using bio-AM, what is acceptable in terms of human enhancement, and issues related to informed consent.

\section{A stepping stone for new ideas}

- In this scenario, the (DIY) communities play a central role for pushing the technology forward. The participants did not precisely define the role these communities could play, but considered that AM for O\&Ps would be of a more experimental character with no profit, mentioning as potential uses body art applications and other very specific uses. The technologies for producing O\&Ps by AM nevertheless play a central role as stepping stone for new innovative ideas. 


\section{Calling for political action}

- The market has changed from medical application to a highly decentralised market where gaming and aesthetic enhancement are the major application areas. Those who benefit are the gaming industry, the cosmetic clinics and the private producers. In reality, everyone is able to print their own orthoses and prosthetics due to open access CAD and cheap, user-friendly 3D printers. Despite the high production of AM O\&Ps, the poor and elderly will not benefit in Europe. The EU will primarily play a role in the export of the technologies while the users are outside Europe - primarily in the US, South America and Asia, where the demand is high.

- The products are marketed with the scope to sell at the highest price while ignoring potential controversies or ethical/societal issues related to the products. Challenges include issues related to dual use and other misuses since there are no regulations on export and application on the technologies. Human right activists are visible in many places, calling for political action.

\subsubsection{Dentistry}

\section{A. Europe outpaced by US-led disruption: Too little, too late}

- US-based economic actors may succeed in achieving a degree of standardisation which would get private health insurance and well-off customers on board. If this happens, US-based actors may outpace European economic actors and establish global value chains that disrupt the relationship between European dentists and dental labs, gradually out-compete European dental labs, and thus move great amounts of the value-creation in the value chain out of Europe.

\section{B. Towards a European model: through regulatory clarity and the support of public insurers}

- If European regulation moves swiftly to clarify the above-mentioned regulatory issues and the roles of different European actors, it may trigger a virtuous cycle. With greater regulatory clarity, e.g. mature safety standards and clear guidance for personal health data processing, it would become viable at once for dentists and dental labs to adopt and apply new production methods, for public insurers to support such new services, and for public and private innovation investors to support Europe-based technology development and the development of new business models.

- A successful European model could include two mutually supportive tracks of development, which would favour the two major actor types - dentists and labs - respectively. The one track would consist in globalised value chains led by European labs and driven by private financing. This track would be dedicated to non-essential and routine applications of advanced 3D manufacturing, such as cosmetic alterations. The other track would consist in localised 3D printing where dentists supported by the appropriate classifications - make use of in-house 3D printing to make higher quality and cheaper emergency operations. The economics of a model supporting such a two-track solution would be synergistic in that investments for the one would benefit the other.

\section{Baseline scenario: Suboptimal development}

- If regulatory clarity is not achieved, it is likely that technological uptake among European actors will slow down and, as a consequence, technology development and innovation will reach only a suboptimal level.

- Such a situation would benefit US economic actors (e.g. US-centric multinationals with ownership in the US and printing centres in cost-competitive countries). Such actors would have the benefit of a home market where there is less risk of regulatory destruction of invested capital and therefore also less hesitancy to invest in uncharted waters. 
- Still, while US-based actors are often quick to move first - banking on supportive regulation down the line -, they also tend to avoid standardisation and harmonisation and therefore tend to reach technically and economically suboptimal solutions.

- If this tendency manifests itself here as well, the end result would be a mixed scenario where lowhanging fruits of centralisation are reaped by some printing centres, but where saturation of additive manufacturing in the European dentistry sector remains lower than one might expect and where the ultimate benefits in terms of higher quality products at lower cost will only partially materialise for citizens.

\section{Regulatory failure: high-tech routines, unchanged emergency response}

- It is conceivable that European regulation fails to become clarified, but that the investments already sunk into technology development programmes do nevertheless succeed in producing higher quality, lower cost production methods, such as predicted by 3D printing proponents.

- Even if European economic actors are not outpaced by their US counterparts, without the proper regulatory support, infrastructures are likely to develop following a "least cost" approach, i.e. globalised value chains where CAD scans are done in Europe and CAM is done in lower-cost global regions.

- This would have the effect of dividing the sector into two areas. One area would be routine and/or non-essential (such as cosmetic) applications of high-tech production methods, where the delays caused by global transport times are acceptable. Another area would be emergency health services where time is of the essence, e.g. when a patient is in pain. These areas would still be serviced by Europe-based dentists and dental labs using more traditional methods. Here, the cost would remain high, quality possibly lower than what would be technically possible, material use would remain high. Nevertheless, since more traditional methods involve less risk from regulation, the net incentive for European actors would be to stay the course for emergency applications.

\subsubsection{Results from the plenary discussion}

Generally, there was a tendency for developing short-term perspectives when addressing already advanced application areas like dentistry, whereas the less developed applications, such as organ printing, lead to more futuristic and imaginative far-reaching scenarios. In the latter types of scenarios, the degree of imagination was also higher regarding what to be expected.

In the group discussions, most of the STEEPED aspects were addressed with emphasis on social, technological, ethical, political and demographic dimensions. Across all groups, there was a discussion on how to ensure that the regulation supports an inclusive society where the technology is also accessible by low income people and where the technology serves medical purposes rather than human enhancement.

The participants acknowledged that regulation is important for directing the technology and provided concrete examples of what policy-makers could do to support the technological development, including establishment of infrastructure, making standards and providing export policies. However, they also stressed the need to identify societal drivers for directing the technological developments. Some of the key drivers identified by the participants were increases in lifestyle diseases (incl. alcohol abuse, tooth decay and diabetes) since these are expected to increase the need for medical recovery. Another driver mentioned was the urge for the "perfect body" which could drive forward the technological development of AM used for aesthetic enhancement.

Since the scenarios were developed with a particular field of application in mind, they address application-specific issues. Despite this, most observations can be used for bio-AM more generally. Particular in the long term or technological advanced scenarios, the participants agreed that it makes 
sense to combine the application areas. It could be e.g. in relation to questions on cosmetic use, export issues, the role of DIY communities, open access policy or demographic aspects.

Overall, most of the questions raised during the group discussions are similar to many issues arising from other technological development, which leads to the question whether it is adequate to separate AM from other technological advancements. Acknowledging that the different fields of technological innovation are closely linked and face many of the same challenges, the participants saw a close connection and synergy between AM and other technological advancements such as AI, robotics and biotechnology.

Finally, the participants saw an important task in adjusting the public's expectations to the technological advancements. The media tend to tell the most spectacular stories that are often also those that have the least hold in reality. According to the participants, it is central to create more researcher awareness in telling the true stories and adjusting the research and technological development to the societal need since we may otherwise expect disappointment in society leading to mistrust and technological opposition.

\subsection{Towards scenarios}

The aim of the envisioning workshop was to develop the results of the horizon scanning into a set of building blocks that could be used for the techno-moral vignettes. In the envisioning workshop, two dimensions were selected that were considered most relevant for the further progress of bio-AM.

1. Technological progress: limited technological progress versus the widespread availability of very advanced AM solutions.

2. Regulation: little regulatory support and low levels of implementation versus a highly supportive regulatory framework and extensive implementation.

Two axes would result in four scenarios (low technology - low regulation, low technology - high regulation, high technology - low regulation, high technology - high regulation) (see Figure ).

The definition of axes led to considerable difficulties, as it turned out in the discussion that regulation may have several dimensions (financial support/funding opportunities, regulation of standards, reimbursement of medical applications, patent regulation etc.) and as the axes influence each other (e.g., funding as a form of regulation will affect, or is maybe the determining factor of, technological progress).

After the $360^{\circ}$ envisioning workshop, a brainstorming with the consortium team was conducted that generated first ideas for storylines of the techno-moral vignettes. For each of the quadrants in the framework, a focus was set on one specific application of 3D printing. For the quadrant of little technological progress and an active state, the vignette focused on 3D printed prostheses for war victims. The quadrant of much technological progress and an active state focused on 3D printing technology to cure the effects of unhealthy lifestyles, e.g. printing new teeth. The quadrant of much technological progress and laissez-faire state focused on 3D printing for body art. The quadrant of little technological progress and a laissez faire state focused on 3D printing for cosmetic surgery and beauty enhancement.

This rough outline provided the initial "scenario space" as a starting point for further development of the techno-moral vignettes.

After the meeting, there was also a round of brainstorming on social drivers and trends (also affecting developments in addition to the two axes regulation and technological development) that might be included as story elements and embed the technology in socio-cultural contexts in the techno-moral vignettes. The drivers and regulatory settings relevant for the final scenarios were then further elaborated and modified in the process of the scenario development, and are listed in Table 1,below. 
Table 1 Drivers and regulatory settings relevant for the final scenarios.

A

- slow development of research and development in life sciences in the EU

- strong data protection and intellectual property rights regime

- active EU role in global development

- unequal level playing field

C

- slow development of research and development in life sciences in the EU

- lack of supportive regulation for bioprinting and $\mathrm{AM}$

- individualisation

- privatisation of healthcare (high insurance fees and limited reimbursement)

- crucial role of healthcare insurance providers

- governments are reactive in terms of regulation and oversight

- strong presence of DIY community, but lack of control and regulation by state or EU

- beauty: urge for the perfect body

- social segregation

- 3D printing for production is standard outside of the medical field
B

- rapid development of research and development in life sciences in the EU

- supportive regulation and policies for bioprinting technologies by national states and EU

- equal access to affordable healthcare based on healthy lifestyle

- rise of welfare diseases, despite prevention efforts

- authority of the state and individual responsibility for health and wellbeing

- access to healthcare predicated on lifestyle criteria

D

- rapid development of research and development in life sciences in the EU

- dissemination of research and development for cosmetic purposes

- restrictive regulation of bio-AM

- individualisation and autonomy for citizens

- privatisation of healthcare services and former government support

- "Attention is everything"

- strong presence of DIY community, but lack of control and regulation by state or EU 


\section{Scenario workshop}

Future developments are unpredictable and uncertain, and many trends and developments have a complex impact on society. Scenarios are a way to gain an understanding of changes in the future in face of the volatility, uncertainty, complexity and ambiguity of change. Scenarios are fictional futures. They are not predictions. Instead, they are storylines that are built upon a thorough analysis of trends and drivers of change, expert opinions and other input. The scenarios presented in the workshop are designed to gain a better understanding of emerging issues and (policy) implications.

\subsection{Methodology}

In preparation of the scenarios, we used the findings from the project so far to draw up a list of main actors/factors and driving forces behind AM as well a preliminary list of uncertainties. We also used the results of the envisioning workshop, especially the scenario building blocks formulated there. In addition, we interviewed several experts and mapped their different expectations of the future.

The techno-moral vignettes were created in three subsequent phases. In the first phase, we used the results of Work Package 1 to draw up a list of main actors/factors and driving forces behind AM as well as a preliminary list of uncertainties. We also used the results of the envisioning workshop, especially the scenario building blocks and the scenario space formulated in this context. By combining building blocks and scenario space, we were able to develop scenario ideas and first sketches of the techno-moral vignettes.

In the second phase, we implemented an iterative process of three summer school sessions at the Rathenau Institute to kick-start the co-creation of the scenarios and receive feedback on our ideas and some further interviews with experts. For each quadrant, a first version of a techno-moral vignette was created. The storyline elements built upon the results of WP1, WP2 and the interviews. The first drafts were presented at one of the summer school sessions. Also the consortium provided input. The later versions of the drafts were discussed with several experts and reshaped until a satisfying version emerged. The drafts were also introduced in some of the interviews. In this way, it was tested whether the techno-moral vignettes helped to initiate a conversation about potential issues. It appeared that the vignettes encouraged the experts to talk about specific issues, which was helpful for the project.

The later versions of the draft were used to identify scenes for the illustrations. The illustrator presented ten illustrations and a logo for each vignette. Each vignette was designed using a specific colour scheme in order to increase identification. Short texts were added to create a storyboard that summarises the scenario and the techno-moral vignette. These were presented in the scenario workshop, where we got useful feedback from potential users of the techno-moral vignettes, which helped us to understand how they are perceived and how to fine-tune them. This feedback stimulated us to further improve the vignettes into their current form.

The scenarios were also developed into first-person stories which function as techno-moral vignettes and which are intended to support Members of the European Parliament and their Committees in understanding potential bio-AM trends and developments in order to anticipate their (policy) impact.

They look at the year 2035 and are built around two main drivers of change: the healthcare system and the role of government and the maturity and availability of 3D printing. In each of the quadrants for this set of scenarios, certain drivers of change dominate. These drivers of change were derived from previous work packages of the project. The technologies included in the storylines of the scenarios were dealt with in WP1. Also, the issues addressed in the scenarios emerged from WP1, WP2 and expert interviews. The scenario development was an iterative process, in which the whole consortium participated. The first version of the scenarios was taken as an input for the scenario workshop. 


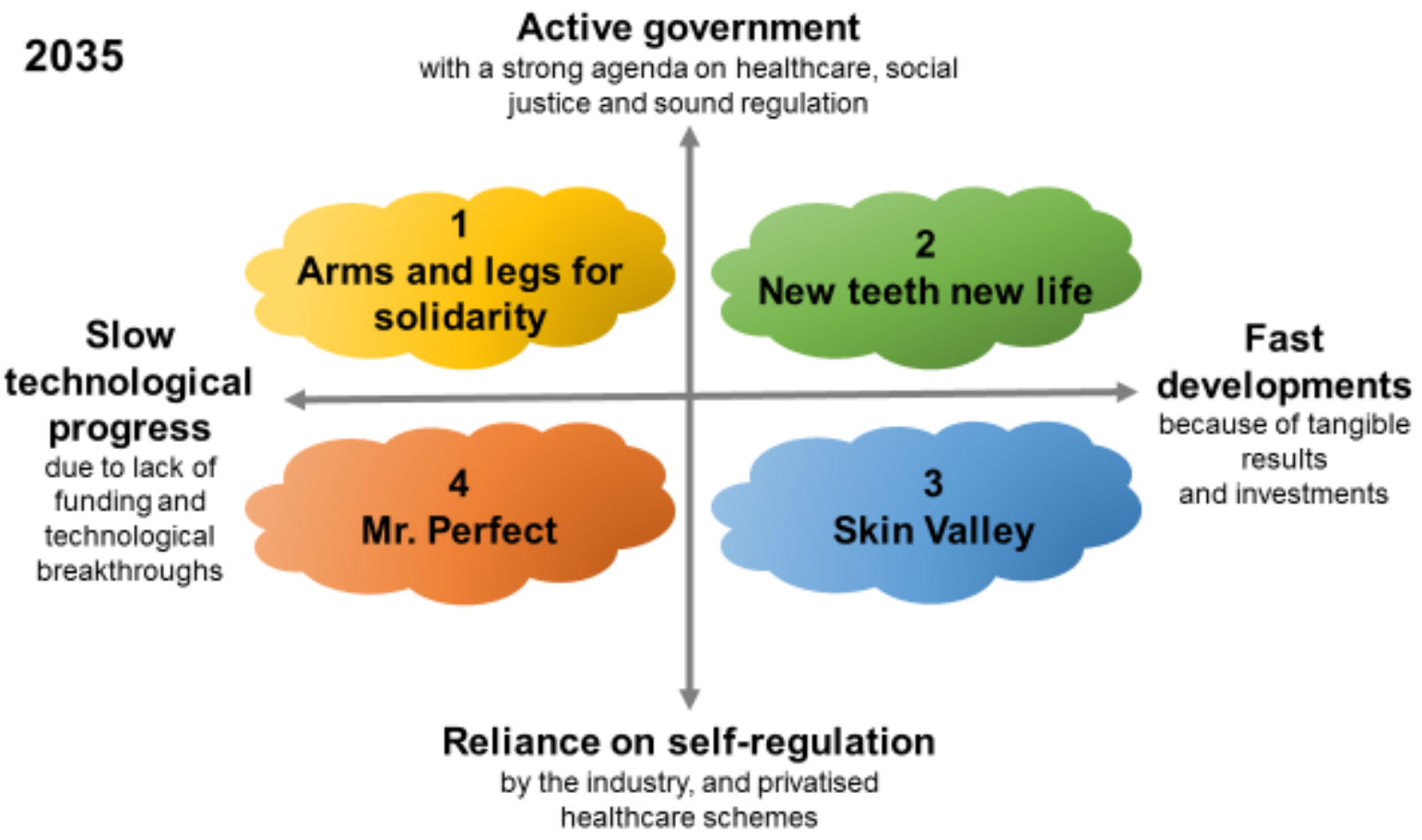

Figure 16 Two axes resulting in four scenarios (low technology - low regulation, low technology - high regulation, high technology - low regulation, high technology - high regulation).

\subsection{Scenarios}

In the following sections, the four scenarios and accompanying techno-moral vignettes are presented.

\subsubsection{Scenario 1: Arms and legs for solidarity}

In this world, innovation has developed much slower in the EU than anticipated in the field of 3D printing. Technologies such as 3D printing did not deliver advanced products and people have to work with relatively outdated technology. National governments are actively pushing for EU regulation on $3 \mathrm{D}$ printing that the medical industry needs to comply with. The EU also holds strong privacy regulations and protects intellectual property rights by all possible means, with serious fines for those infringing the rules and regulations.

However, these rules only apply to EU member states and EU-funded organisations, causing an uneven level playing field with organisations funded outside Europe.

European organisations play an active role in supporting post-conflict regions and developing economies globally. The lack of regulation in these countries and the urgency of the issues the CSOs have to deal with create a quite different playing field for the development and use of 3D printed medical applications. For instance, European CSOs work on providing 3D printed prostheses to children in war-ridden countries - programmes that gain a lot of public sympathy, without much concern about where these prostheses come from. Transparency is an issue, as detailed reports of the achievements of these CSOs are not always available.

Drivers in this world are:

- slow progress of research and development in life sciences in the EU;

- strong data protection and intellectual property rights regime;

- active EU role in global development;

- unequal level playing field. 


\section{Techno-moral vignette}

\section{What happens when innovation funds are inadequate?}

In this scenario, an EU-funded civil society organisation (CSO) provides prostheses to kids without ensuring adequate protection of their medical data or its own intellectual property.

"There you go. This tiger leg is yours now." Carla worked for Hearts4Kids, an CSO that furnished kids with prosthetic limbs. She was proud of eight-year-old Mo, who was taking his first steps with his new prosthetic leg. It was decorated with a tiger stripe pattern, just like Tigo, the main character of the blockbuster film by FM Company. Every kid wanted to have Tigo things these days, and Mo was no exception. Like many young children in his country, he had

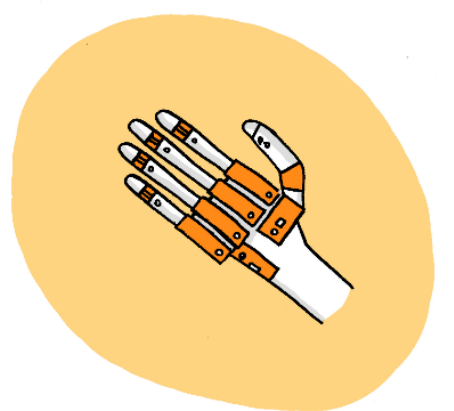

Scenario illustrations by LUC - le Laboratoire d'Usages Collaboratifs lost a limb playing in an area covered in landmines. Civil war had raged across the country for many years, with rival militias fighting over access to water and dominance in the main cities.

Hearts4Kids helped as many of these kids as it could. Their prosthetics were not very fancy, and the electronics that controlled the limbs were far from perfect. Putting on a shirt remained difficult, for example. Their 3D scanners were outdated and measured only a limited number of anthropometric data points without being tailored to each individual patient. The printers were slow and the materials fell short in terms of strength, weight and durability. But their prosthetics were cheap to produce (they simply printed them on site) and many of the materials were recycled and reclaimed. That was a big plus because the children needed new, bigger limbs when they grew older.

Hearts4Kids had its roots in Belgium and was funded by the EU. Some local businesses were critical of these subsidies. They claimed that the EU was disrupting the local prosthetics market. On the other hand, some local firms were themselves collaborating with big

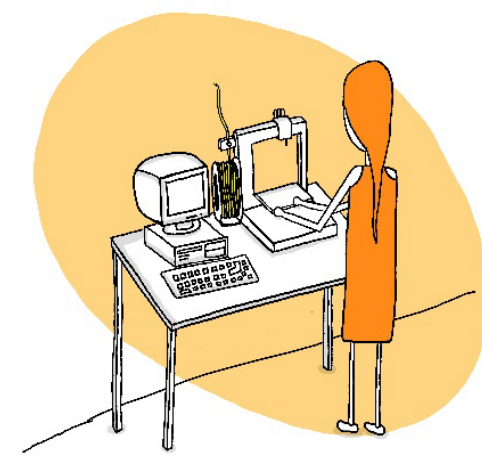

Scenario illustrations by LUC - le Laboratoire d'Usages Collaboratifs overseas companies. One of these was Roko, once a small firm that had expanded quickly thanks to its partnership with an Asian company. It designed amazingly effective prostheses for kids. Like many local companies, Roko was a firm advocate of open source technology. This made it interesting to Asian developers who worked with new materials and advanced electronics. Kids with Roko legs could even run. Their prosthetic limbs were also decorated with the Tigo pattern but without being licensed by FM Company. Hearts4Kids would never engage in this type of copyright infringement. To be honest, they didn't need to: companies knew that collaborating with Hearts4Kids boosted their reputation and so they offered the designs for next to nothing. 


\section{No EU funding without respect for intellectual property}

A few days later, Carla attended a staff meeting. Roberta, her boss, said she had strong evidence that local companies were using their own and other design technology to print Tigo designs and that they had been doing so for years. How these companies got hold of these designs was not completely clear to Heart4Kids because the CSO had taken protective measures. Roberta feared that developers, for instance hardware or electronics firms, would no longer want to collaborate with Hearts4Kids if it was unable to protect their intellectual property. And without these developers, Heart4Kids would not be able to deliver prostheses, making it almost impossible to attract EU funding.

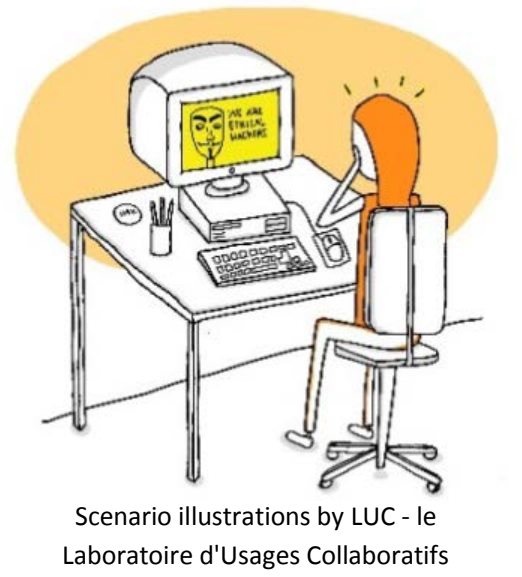

Earlier that week, Roberta had also found out that their computer system had been hacked. An activist group had published their huge database of anthropometric information on thousands of kids. The group claimed that they were ethical hackers who wanted broader access to prostheses. The data could be used for research and development, but more importantly, the activists wanted to make a statement about the need for open data. All children should have access to prosthetics, the group claimed, and open access data was an important step towards expanding accessibility and stimulating innovation.

Although Carla sympathised with this idea, the hack was very damaging to Heart4Kids' reputation. To receive EU funding, the organisation had to comply with strict privacy and data protection rules. The hack showed how risky it was to own a database containing personal information and would likely fuel the ongoing debate about CSOs and private organisations collecting and storing personal data. Many people feared that companies would exploit these data, and the incident might also stir up the old controversy about Heart4Kids making prostheses that had not undergone safety testing in the EU. So far, their response had been that Heart4Kids did not work in the EU and served people in need. Testing would make affordable prostheses impossible.

Six weeks later, Carla started her last day of work at Hearts4Kids. The past month had been hard for everyone at the CSO. Many politicians and opinion makers had been calling on the organisation to cease

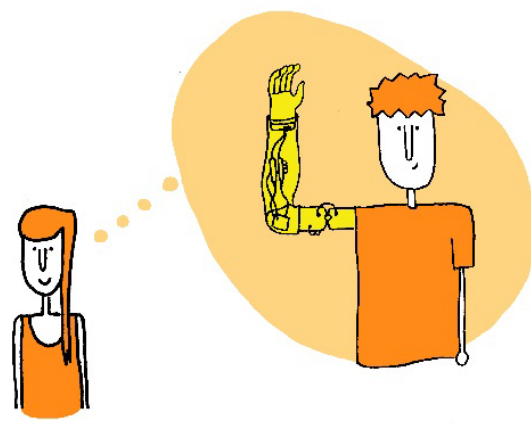

Scenario illustrations by LUC - le Laboratoire d'Usages Collaboratifs its activities. Others had argued that a new open data approach would be an opportunity to collaborate with local companies. Still others believed that the EU should have provided CSOs like Carla's with more money so that they could attract developers and invest in data security. The reality was that local firms that worked with open data, like Roko, were more successful at producing innovative prostheses. And there was good news for Carla: the people at Roko had asked her whether she would be interested in joining their team and continue helping kids like Mo. 


\subsubsection{Scenario 2: New teeth, new life}

This is a world focused on health and wellbeing. The EU has stimulated life science research for regenerative health to a large extent, and many public and private initiatives have benefited from the extra funding and support. National governments provide several support programmes to enable equal opportunities by helping people improve their lifestyles and providing high quality care. At least, for those who are willing and able to take responsibility for a healthy lifestyle. Many are still struggling with this. There is a rise in welfare diseases despite prevention programmes.

While health is seen as one of the most important values in life, and prevention a key responsibility of the national and EU governments, this also means that government bodies decide what healthy behaviour is. Reimbursement of health costs is based on lifestyle criteria, and those who do not comply with the state rules will have to look elsewhere for care. Given the high number of welfare diseases, the healthcare system only serves the relatively healthy who are willing to actively work on their health and wellbeing.

Technology progress has benefited from sustained investment in research and development. 3D print technology is big. For instance, 3D printed teeth are widely available due to lower material costs and less waste, even though these are considered fake and fashion items by some. And there is 3D printed customised medicine.

Also other technologies are developed like Artificial Intelligence and several converging technologies that are being applied to healthcare and wellbeing. Actually, every aspect of life has been automated, which has resulted in virtual assistants for everyone. Most people know how to use these technologies and they are familiar with customised solutions, printed at home or in clinics on demand, and easily adopt new applications. Some use more of these technologies than others, but in general it is an integral part of daily life - which is not to suggest that welfare diseases and lifestyle issues are a problem from the past. On the contrary, in 2035, there are, as ever, people who find it hard to develop a lifestyle which complies with the formal definitions of what is healthy.

Drivers in this world are:

- rapid progress of research and development in life sciences in the EU;

- supportive regulation and policies for bioprinting technologies by national states and EU;

- equal access to affordable healthcare based on healthy lifestyle;

- rise of welfare diseases, despite prevention efforts;

- authority of the state and individual responsibility for health and wellbeing;

- access to healthcare predicated on lifestyle criteria.

\section{Techno-moral vignette}

What happens when innovation funds are inadequate?

In this scenario, we meet Ben and his son Davy. Life is hard for them. They are told that new, 3D printed teeth will improve Davy's health and his life in general ("The technology is getting better and better").

For weeks now, he'd been sleeping poorly. Ben's pale face looked sad and worried. "Maybe it's all my fault," Ben murmured to himself. Davy had often been alone at home. "I should have been there for him more." And then a deep sigh. "If only I didn't have to raise him all by myself. I don't blame him for eating crisps and drinking fizzy drinks." A few years ago, the doctor had given

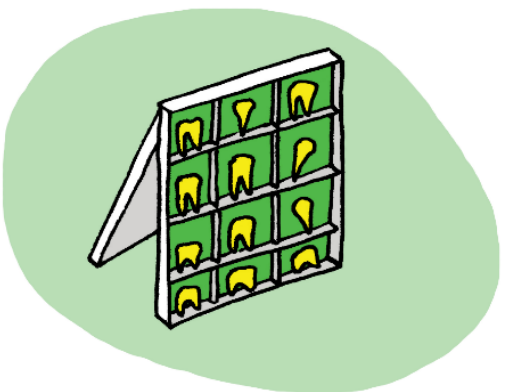

Scenario illustrations by LUC - le Laboratoire d'Usages Collaboratifs Davy a personalised diet, customised to his personal genetics to improve his health and help him get 
fit. The diet consisted of 3D printed food and supplements, along with an exercise regime and an app for personalised motivational messages. But after six weeks, Davy gave up. He said the food was fake and not as tasty as what he was used to. The exercises - he just didn't feel like doing them. Sometimes Ben wanted to look away when he saw all the empty bottles of energy drink in his son's room. Davy's diet was ruining his appearance, but also his teeth.

Davy had finished school last summer. It made Ben happy, but he was also worried. Would Davy ever find a job? He still had his digital coach, part of the support programme that he'd been enrolled in, but he hardly ever listened to it. And would he ever get a girlfriend? Davy, who had been overweight all his life, didn't dare to open his mouth in public. He wouldn't admit this to his dad, but his breath smelled awful. Almost all of his teeth had turned into little grey stumps. He was embarrassed, but he wasn't fond of the dentist either.

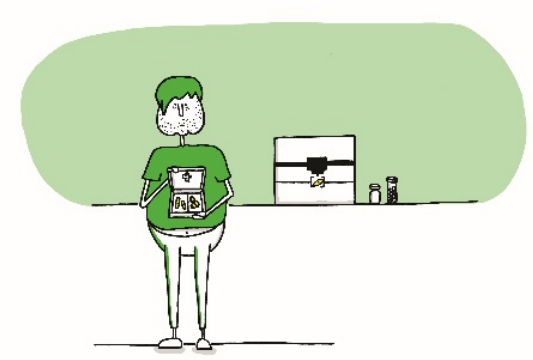

Scenario illustrations by LUC - le Laboratoire d'Usages Collaboratifs

Ben had also struggled with some health issues - high blood pressure, high cholesterol levels, like many other people. In addition, he had reduced liver function, a genetic defect that had been in the family for generations. The doctors had given him a little device to measure his blood values. He entered the test results into a machine that automatically adjusted the level of medication he needed. The pill that came out of the printer was enough for that day.

He wanted a better future for Davy. He himself had been in and out of hospital for a long time. Being heavy had damaged his

knees. He got new 3D printed knees some years ago, but since then he had turned grumpy and started drinking heavily. He had heard about 3D bio-printed liver tissue that would extend the lifespan of an unhealthy liver before transplantation became necessary. There had also been a few major success stories involving engineered liver tissue. Cultivating tissue from the transplant recipient's own cells removed the risk of rejection or the need for immunosuppressants. The rise of 3D printed organ tissue and organs grown in transgenic pigs had led to a decline in the number of human organ donors. But all these innovations did not help him much. The doctors had ordered him to adhere to a strictly sober lifestyle before they would even consider cultivating new liver cells. And that was just not how he wanted to live his life.

\section{Additional healthcare, funded by the EU}

Ben thought back to the health official from his son's former school that used to visit them to inspect the home situation. The health official had told them about this new programme, funded by the EU, to provide additional dental care to those who needed it. The cost of this treatment had gone down dramatically. It was thought that making this service affordable for a large group of people would improve their job opportunities and life satisfaction. Apparently, this was part of the EU action plan to improve public health, employment and quality of life. Modern technology meant that your regular dentist could fix your teeth as good as new in just one session. The new 3D printed teeth were much cheaper than older techniques, and they wasted much less expensive material. On top of the EU programme, the national government in his country would cover the entire dental bill. In other countries, people still had to pay for this themselves.

This was good news. Life had been difficult enough so far. Many companies had reduced their workforce over the years because everything was automated now. Computers and robots did the work that people used to do behind the scenes. Young people like Davy hardly had a chance, even after finishing their education. There was always a healthier, better-looking candidate. Unlike Davy, most people worked hard to look good and be successful. Looking good was very important because most jobs involved customer relations. Research on appearance and social status had shown that something as straightforward as good dental health really mattered in the job market. Besides, dental health issues 
were a well-known risk factor for many other health problems, including heart disease. Good teeth could therefore make a major contribution to people's health and their opportunities in life. Indeed, Davy's digital coach always reminded him to brush his teeth - in vain, evidently.

\section{A new set of perfect teeth}

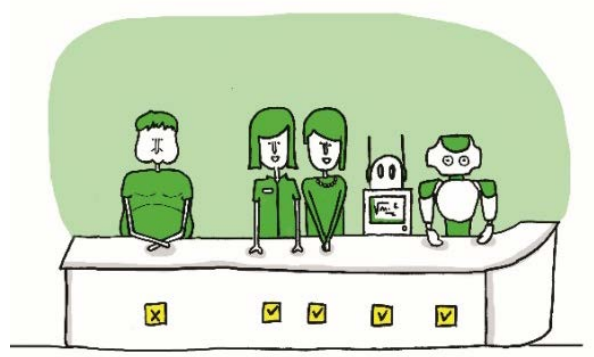

Scenario illustrations by LUC - le Laboratoire d'Usages Collaboratifs
The next day Ben called a dentist. Not to make an appointment for himself - the thought of it made him shiver - but for Davy. He and Davy hadn't been to the dentist for a long time, so he was surprised to hear all the things they were now able to do. The digital voice on the phone explained that he could visit the clinic to learn more about the procedure. At the clinic, he learned that a robot doctor would make a scan of the inside of Davy's mouth so that the dentist would know exactly where to position his new teeth. Davy would also have a more precise bite, with the upper and lower teeth matching perfectly. The dental profession had accumulated a database of thousands of patients with a perfect bite, so dentists knew exactly how to shape new teeth for Davy. After a little puff of anaesthetic and a tiny injection, he would feel nothing. Then the work would begin. While they removed the old teeth, new ones would be printed on the spot. They would first place each of the customised implants. Once the printed teeth were ready, they would only need a bit of polishing and could be attached to the implants straight away. The result would be a new set of perfect teeth made from almost-natural material. All Davy's teeth would line up precisely where they needed to be.

"Is it safe?" Ben asked. "Absolutely! More advanced methods are common in other countries outside the EU, where they're even experimenting with printing real teeth from stem cells. But this procedure is safe, we have a lot of experience and we also have the necessary certification," the man at the desk explained - or was it a man, Ben wondered. "You might have seen scary pictures about futuristic procedures in other countries. It is nothing like that here in Europe. We have high standards. Not every new technology is marketed immediately. But once it gets approval, it's safe. The government's 'new teeth, new life' programme is so successful that we do this procedure every day, and the technology is getting better and better all the time." It sounded great. Ben was sure he could convince his son to have the procedure done. And he was right, in the end.

\section{Natural beauty or not?}

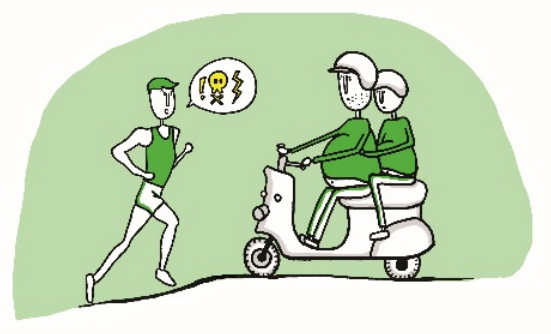

Scenario illustrations by LUC - le Laboratoire d'Usages Collaboratifs

"Come on in." The digital dental assistant's warm greeting was just what Ben and Davy needed. While Davy was in the chair, Ben scanned through newsfeeds. He read about new technologies that he barely understood, something about using your own stem cells to regrow a missing tooth. He was happy with Davy's treatment, but this also sounded exciting. One of the headlines read "Natural beauty or not?" The article was about people who didn't want to date someone with artificial teeth. Ben decided to ignore it. He had more fun reading about how to transform your teeth into a work of art. Some people apparently had sensors in their teeth that were connected to their mobile device. They had an app that allowed them to project a different work of art onto a tooth made of responsive materials. "That's crazy," he thought. He also read about the use of bite marks as a new identification tool, in addition to iris scans. With everything that was going on in his mind, he had closed his eyes to all this news. All he wanted to think about was Davy's new teeth. 
But now he read that a lot of people blamed families like his, something he himself had experienced. "These people live like parasites," read one quote. "They smoke, drink and eat junk food. They get treatment without improving their lifestyles. And now we're all paying for it." He was used to critique, but this felt very unfair. And it wasn't even true.

When the dentist was finished, Ben could see by Davy's face that the whole procedure had not been as bad as he had feared. He looked happy. Davy gave him a big smile. All his teeth were there in a clean, tidy row. The start of a new life, Ben thought. Driving home on their scooter, Ben wondered: maybe now it was his turn to change his life and get a new kidney. He wasn't that old yet, after all. He gave Davy a big smile: new teeth, new life! Then he almost hit a jogger, who just managed to leap to safety. "Hey, you fat ugly leeches," the man yelled at them. "Get out my way!"

\subsubsection{Scenario 3: Skin Valley}

Globally, technology has made great leaps forward, in particular in biotechnology and biomedical research. The EU has invested heavily in research, education and innovation in order to become more competitive. However, regulatory frameworks have slowed down applied research and development in Europe.

This has hampered the dissemination of knowledge and technology breakthroughs in the market. EU citizens travel to other parts of the world, like the US, with more favourable conditions and better access to medical treatments and services, or to the European metropolitan hotspots were local DIY communities try to keep up with global innovation. While licensed research labs have limited options due to strict regulations, the DIY labs have much more freedom and are poorly regulated - attractive to some, but surely not to everyone. Regulatory oversight has proven to be difficult in such less formal settings, but there is a growing group of citizens believing in the value and power of these DIY communities.

Some of these communities consist of so-called "body hackers": people who modify their bodies for aesthetical, practical or other reasons. This scene has matured over the years, and can be seen as the next step in body art, such as tattoos and piercings. Attention is everything in this world. While government health services have declined, cosmetic applications are accepted widely and body art within the DIY community is a hype. The EU tries to control some of the DIY practices, but is unable to stop this because there is no authority or mandate at this level.

Drivers in this world are:

- rapid progress of research and development in life sciences in the EU;

- dissemination of research and development for cosmetic purposes;

- restrictive regulation of bio-AM;

- individualisation and autonomy for citizens;

- privatisation of healthcare services and former government support;

- "Attention is everything";

- strong presence of the DIY community, but lack of control and reguation by state or EU; 


\section{Techno-moral vignette}

\section{What happens when body artists cultivate cells in a lab?}

In this scenario, twins Rubi and Sai, 18-year-old vloggers from Denmark, travel to a DIY studio in Berlin to get a colourful body jewel ("I'll take a tiny sample of your cells and then we'll do everything here in the lab").

"Wow! I really want that," Rubi said to her brother Sai. "Do you see what they have on their arms? That's what I want for us too!" She pointed to something glittering on the arms of the famous twins that they were named after, Rubi and Sai Cole, the children of the singers Bella May and JilZ.

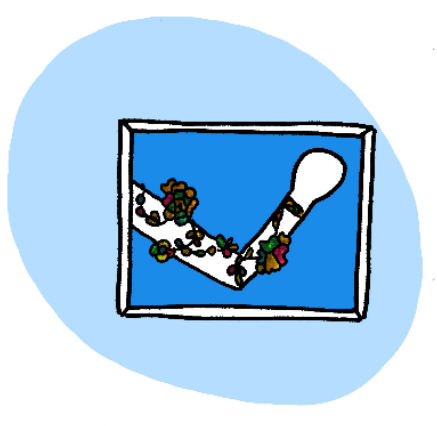

Scenario illustrations by LUC - le Laboratoire d'Usages Collaboratifs

Rubi pointed excitedly to an audio-visual projection of the other Rubi and Sai, who were proudly displaying what appeared to be a new kind of tattoo. Both had a small flower on the inside of their lower arm, Rubi on her right arm and Sai on his left. The flower was colourful, but it couldn't possibly be made of the usual tattoo ink. Rubi immediately recognised them as body jewels, 3D tattoos made from the wearer's own skin cells. Cultivated in the refined lab environment of a body art artist, the cluster of cells were transformed into a beautiful three-dimensional flower with colours and glittery reflections that were impossible to make with plain old tattoo ink.

Sai was intrigued. Rubi had shown him some photographs of people with body jewels. Now that he saw the other Rubi and Sai sporting body jewels, he thought it would be amazing to have them too. All their online friends would love it. Body jewels were new and edgy and only a few people had them. It would be a fabulous opportunity for their vlog, he thought. They would hit a million viewers for sure, and showing that body jewels were not only for the rich and famous but affordable for normal teenagers would attract a great sponsor.

\section{A new generation of tattoos}

Rubi had always followed body art trends. Their parents had tattoos all over their arms and they were still very happy with them. For as long as she could remember, Rubi felt proud of the strong presence that their tattooed bodies projected. Now she could have something even better.

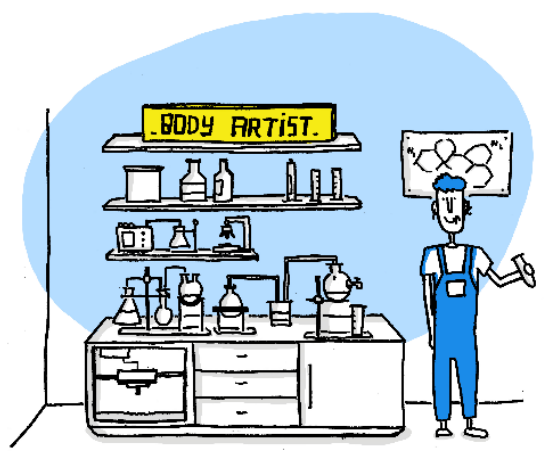

Scenario illustrations by LUC - le Laboratoire d'Usages Collaboratifs

The technology was originally developed to treat burn victims. Skin replacement methods became better and better. Researchers also used skin and other cells to run tests without using laboratory animals. It was even possible to print deeper layers of tissue, with nerves and blood vessels. Artists had started using the technology outside the lab to create body art that could not be made in any other way. As far as Rubi was aware, there were only a few artists doing this. They had formed a small, global DIY community that shared designs, experiments, results and failures.

Medi Valley was where it had all started, not in the official medical facilities but in "artist-owned" labs at the edges of the valley. It was too expensive for them to travel there all the way from Denmark. Local consultants would do the intake, but they would still have to fly to Baltimore for the actual procedure. The cost varied from one place to the next, and you could easily end up in a lab with a dubious reputation.

She knew that some body artists in Europe had adopted the new technology and now designed body jewels themselves and experimented in DIY labs with colours, artistic effects and new forms. One of 
them was in Berlin, where she hoped they could both get an experimental jewel for next to nothing. The studio she'd heard of was part of the underground art scene, but she'd read some great reviews about the artists behind it. They had refined their skills by practising on their own bodies. Karsten, the studio's founder, used to work in a public research lab, but he had wanted to go further and was now probing the limits of legislation.

\section{"Nothing more than a mild, local anaesthetic"}

A few weeks later, the twins found themselves at a location just outside Neukölln. Rubi took it all in. The studio, or lab if you want, had all sorts of boxes on shelves with tubes going in and out. Karsten, a tall guy with at least twenty rings pierced through his lips, was taken by Rubi's enthusiasm. He liked the young duo. "It's nothing more than a mild, local anaesthetic," he said. "I'll take a tiny sample of your cells and then we'll do everything here in the lab." He gestured enthusiastically as he talked about the flower that he wanted to create for Rubi and Sai. He showed them his own jewel, just above his waistline.

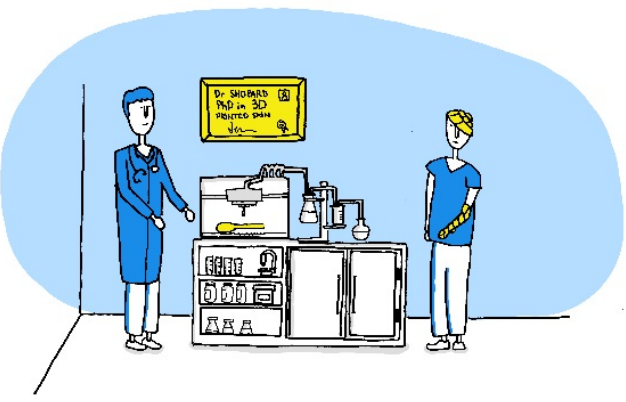

Scenario illustrations by LUC - le Laboratoire d'Usages Collaboratifs "You see, it's nothing like a normal tattoo. It has layers and the colours are much more intense."

The four months needed to prepare the jewels seemed endless to Rubi. The skin cells first had to be modified and multiplied before they could be printed in the shape they had designed with Karsten's help. Sai was also a little nervous. The famous Rubi and Sai had attracted quite a lot of attention with their body jewels. And there were people with similar jewels all over the internet now. Would they be in time to inspire their online friends and attract more followers to their vlog?

Finally, Rubi and Sai returned to Berlin. Karsten would attach the jewel to the skin with some sort of bio-glue. When he showed the twins the two jewels, they could not believe their eyes. They looked even better than the three-dimensional figure on Karsten's body. "I've applied a new technique to let the green come out even better," Karsten tells them proudly. When the twins walked out of the studio, their arms were still covered in bandages. "You need to protect it for at least a week," Karsten told them, but they could hardly wait to show their mum and dad.

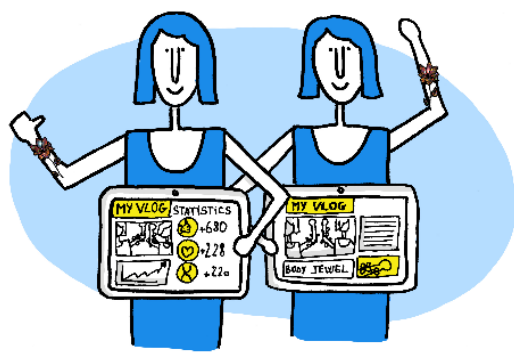

Scenario illustrations by LUC - le Laboratoire d'Usages Collaboratifs
Back at home, dad was more excited than Rubi and Sai had expected, but also a little concerned. Under the bandages, the body jewels did not look as incredible as Rubi claimed. Dad found them a little weird, almost like a fibroma. But Rubi wouldn't listen. A few days later, she revealed the body jewels on their vlog. Just as they had predicted, the views poured in. "Over 10,000 views within an hour." Karsten would like that too and gain some customers in return for his help. Sai was also delighted. The views and the likes gave them a lot of data about their viewers that he could easily sell for a lot of money.

\section{Horrible infections}

Dad's concern was not unfounded. He had seen pictures of horrible infections on the internet. But that wasn't even the worst of it. Some online articles suggested that these jewels could develop into cancer, with cells dividing and migrating. Or the material might travel to other parts of the body where it could do damage. "Would it be possible to remove the jewel?" he wondered.

The National Health Inspectorate had launched a debate about body jewels even before the EU could ask for expert advice on regulatory restrictions. Without robust knowledge about the long-term effects, 
the NHI was already advising against using these techniques in DIY studios. It emphasised that the technology was in its infancy and that the health risks were unclear. One of the questions raised in the public debate was who should pay for medical care if something went wrong. Many people thought that public money shouldn't be used to treat people who had allowed themselves to be used as guinea pigs. Some wanted to forbid body jewels altogether. Others thought that people had the right to do what they wanted with their own body, and that proper regulation should prevent the use of hazardous materials, inappropriate facilities and high-risk procedures carried out by people without the right medical training. For dad, these were not real issues. He was simply worried about his kids.

\section{"The EU has no say in this"}

Mum was also increasingly unhappy about the twins' body jewels. She followed the debate and read that politicians in Brussels were talking about banning consultants for overseas beauty treatments and punishing underground DIY parlours. Their fear was that criminals would use this technique to copy fingerprints and sell them on the Dark Web. However, legal experts explained that it was extremely difficult for the EU to restrict DIY practices, especially if studios offered their services for free to friends and acquaintances or as part of a barter system. And because authorities in other parts of the world were more lenient about commercialising these technologies for body art purposes, regulation was even more difficult.

The more mum heard, the angrier she got. Health and safety had not been a priority in this field, much less so than in the public medical sector. There was no evidence that the procedure was safe - in fact, quite the contrary. Body jewels might have been a Medi Valley spin-off, but what the colouring and layering would do to the property and safety of the printed skin had never been clinically tested. "If this is true, I want yours to be removed immediately," she screamed at Rubi and Sai.

Rubi tried to calm her down: "Don't worry, mum. The studio also does all sorts of regular implants that track your health and that kind of thing. These have been available for years now and people like Karsten know what they're doing in their studio." But mum was not convinced. She continued to worry about the risks. The National Health Inspectorate must have good reasons to be cautious about body jewels.

\subsubsection{Scenario 4: Mr. Perfect}

Healthcare has been privatised in many EU countries. People who can, and are willing to pay for it, get high standards of care. Public research funding in general has become less and less over the year and that includes the research budgets in health. Stringent choices were made and, due to lack of positive results, bio-AM has lost its chances to make its promises real.

The big public resistance against the engineering of printed organs did not help either. In general, the ethical debates about life sciences developments have hindered technological progress in health. For innovative health companies, the EU is not an attractive market anymore. They find the regulation rather outdated, public opinion is volatile and the consumer market is not really willing to adopt. EU has become a late adopter.

This in contrary to other parts of the world where research and development on cancer and degenerative disorders has continued and has resulted in advanced therapies and a growing market in human enhancement.

Some politicians claim it is because of the costs, others say these technologies affect traditional European ethics. The outcome is the same: they are not covered by public health schemes. Thus, the clinics in the EU who work with these technologies serve only rich people who can afford. For some of them, these health technologies are much more life style technologies.

Drivers in this world are:

- slow progress of research and development in life sciences in the EU; 
- lack of supportive regulation for bioprinting and AM;

- individualisation;

- privatisation of healthcare (high insurance fees and limited reimbursement);

- crucial role of healthcare insurance providers;

- governments are reactive in terms of regulation and oversight;

- beauty: urge for the perfect body;

- social segregation;

- 3D printing for production is standard outside the medical field.

\section{Techno-moral vignette}

What happens when money buys faces?

In this scenario, François replaces his nose by a 3D printed version of a celebrity's nose. After a while, and after the celebrity finds out, he doesn't feel so confident anymore.

François was a rising star, the head of a small, specialised unit at one of the most prestigious FinTech companies at La Défense in Paris. He had a fifth-floor apartment with view of the Eiffel tower, he was goodlooking, and he dated a lot, but nothing serious.

François inspected himself in his bathroom mirror and felt truly smitten. He looked stunning in his new haute couture suit by his favourite designer. He'd been told that he resembled the famous

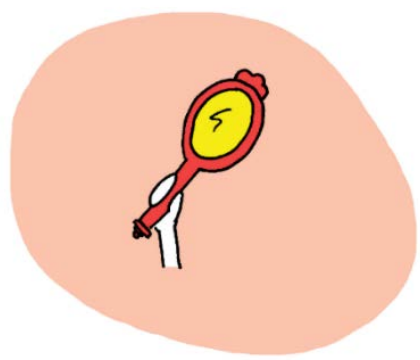

Scenario illustrations by LUC - le Laboratoire d'Usages Collaboratifs male model, Giovanni Borsa, who represented the designer's label. François liked this label a lot, especially the shoes with custom $3 \mathrm{D}$ printed soles.

His collection of hair and skin products was impressive. François had always treated his skin with the greatest of care. Skin cancer had reached epidemic proportions in recent years. It had become very easy to diagnose. There had been many public health campaigns during the years, but many people neglected the warning signs until it was too late. Really stupid, he thought, smartphones warned you if they detected signs of skin cancer on your selfies. What more did these people want? He found it ironic that despite good diagnostics, there had not been much progress in treatment.

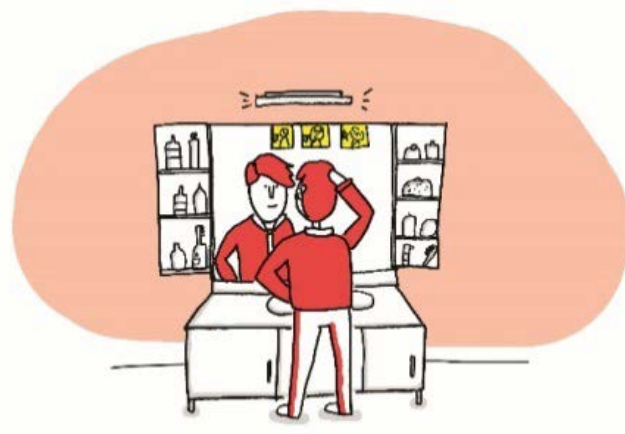

Scenario illustrations by LUC - le Laboratoire d'Usages Collaboratifs

A cure for cancer had always been the holy grail. He wondered why technology had delivered custom-printed lightweight airplane parts and more sustainable biofuels, but not very much in the way of better healthcare. It turned out to be much more difficult than researchers had anticipated. And politicians were always talking about the ethics and the need to proceed with caution. Some people felt that things like bioengineering and tissue printing were unnatural and should be banned. Crowds had taken to the streets of Paris in protest. As a result, high-end research that seemed promising in the past had been discontinued. Many people were also struggling with the costs of privatised healthcare.

He put on some sunscreen and inspected his face. From the side, he really did resemble Giovanni Borsa, like two peas in a pod, but from the front he clearly had a different jawline, cheekbones and nose. That bothered him. On his online dating profile, he only used pictures showing his face from a perfect angle. He also used a fake name: Giovanni. It was his little trick to make women think he was the famous model. And often, it worked. Most of the time he told them straight away that he wasn't that Giovanni. 
Still, it gave him a little head start and worked as a nice conversation opener. His phone beeped. Another match! Nathalie, female model. Gorgeous! She messaged him: "Giovanni, is that really you?" He smiled. Maybe he should make the resemblance perfect.

"Choose your favourite celebrity's nose"

Later that morning, at the office, he could not resist the thought and looked up the beauty clinic where he had his hair replacement therapy and eyelid correction four years ago. They would surely have new techniques by now. The clinic's slick website said: "Have your dream face, choose your favourite celebrity's nose." The website showed how people could have the same nose as their favourite singer or actress. 3D print technology could create a bone implant with perfect precision - much better than the old-fashioned rhinoplasty that François had undergone years ago. The clinic claimed to have the best data, with the most precise data sets on celebrities all around the world. They not only printed nose implants but also cheekbones and jawlines. François got very excited. Would they have data on Giovanni Borsa too?

Although some of the pictures of the nose replacement procedure looked scary, François knew that he had to go through with it. It took only three clicks on the website to make an appointment. Within a week, François found himself at the fancy clinic. An attractive doctor explained the process. There was nothing to be scared of. Basically, it was like any other plastic surgery. The only new bit was that they would print the cartilage for his nose and jawbone during the operation, in a shape that would make the perfect nose for him - the nose of Giovanni Borsa. And they would use a scanner to determine the exact location of his facial nerves and veins so that the nose fit perfectly. "You must understand that this is not for everyone," she continued. "Here is an overview of the costs. Prices depend on the number of implants needed for the best result. And we need you to take the psychological test." François wasn't bothered about the test, but the price tag made him swallow and hesitate. "Alright, I'll do it. I have to do it. I need this," he convinced himself. After all, he had done well on the stock market and expected a nice bonus. A month later, he would undergo the procedure. He would spend a week recovering in the clinic. "We'll treat you well," the doctor promised him with a smile.

\section{"That much money for a nose?"}

And they did! The week in the clinic was almost like being in a holiday resort, although he felt pretty awful the first few days with his face all swollen. On the first day, they had taken very precise measurements of his facial bone structures and matched these with the data on Giovanni Borsa's facial features. To reduce the swelling and improve the result, they had even given him a 3D printed facial mask to wear most days. He hadn't felt so undatable in a long time. With a big bottle of painkillers tucked into his bag, François was ready to go home. He had to take off for another week to recover. His face was still a little bit swollen, but the doctor told him the swelling would slowly subside and his bruises fade.

Two months later, François was on another first date. Eva and he had really hit it off and they lingered in the café. He told her more than he'd intended about his recent upgrade. "What!" Eva exclaimed. "You totally tricked me!" She was obviously upset. "I didn't know they could do that." She gave him a strange look. "Why would anyone want to do this? Why would you want to put so much money into this?"

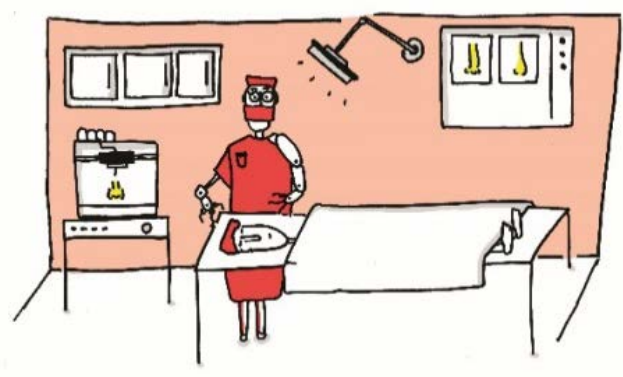

Scenario illustrations by LUC - le Laboratoire d'Usages Collaboratifs

An hour before, she had told him about her mum and dad. They were both very ill. Her dad had skin cancer and her mum had colon cancer. For years she had put all her hope into the new treatments that the doctors were promising. They always said that they would soon be able to grow new intestines in 
the lab and cure her mother. But it never happened. The research on 3D printed body parts had stalled, they told her. And now François was saying that they could actually print noses and faces, just for fun!

And of course, her dad should have checked his moles much sooner. Her app had even warned her about his skin when she posted a family picture. And there had been all those public health campaigns. Now it was too late to treat him because he didn't have the money. There were therapies abroad but they were far too expensive. The ageing population meant that privatised healthcare costs were skyhigh and they could not afford the travel and clinics on their own. Besides, dad had a high-risk profile and ignored the warning signs, so the insurance would cover only a fraction of his medical expenses. And now she was sitting here with François, who bought the most advanced treatment for a bizarre reason. "Is this what you spent your money on? While my parents are told that they're incurable just because they don't have the money? It's so unfair!"

\section{"Our faces are our data"}

The next morning, François woke up a little sad that Eva had left soon after he had told her about his facial surgery. But he cheered up when he saw his beautiful face in the mirror.

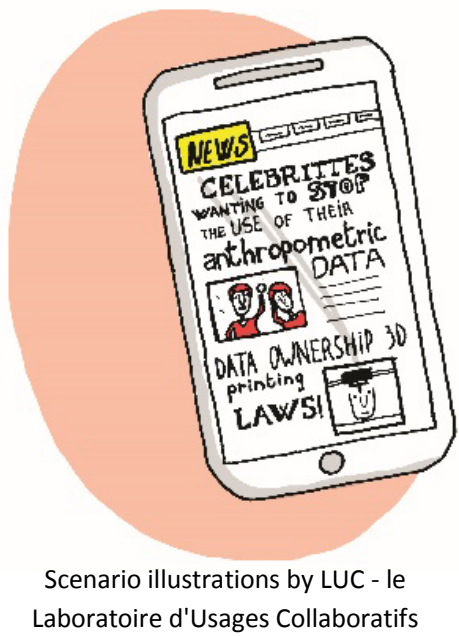
legal action. All of a sudden, François no longer felt so confident about his good looks.

\subsection{Findings}

The project team explained the scenario method and presented four scenarios by going through illustrations matching the storylines. Afterwards, participants worked in two groups, moderated by a member of the research team, on two of the scenarios. Both groups should read one scenario, digest the main idea of it, list the main issues and improve the scenario by adding and changing issues that were in the scenario. For each of the steps, the participants were asked to write their thoughts down on a post-it; these were later grouped and discussed. The same thing was done for the second scenarios.

In a final ranking exercise, participants could divide three points (using stickers) over the element, thought or issue they found most important. Table 2, below, presents the result of this exercise and also indicates in which scenario which issue is emerging. This was discussed in a plenary discussion. The session was then closed with the question: "What do you take home from today?" 
Table 2 Summary of the key points ranked in the discussion. The " $X$ "s in the coloured columns show, in which scenario which issue emerged.

\begin{tabular}{|c|c|c|c|c|c|}
\hline \multirow[t]{2}{*}{ Issue } & \multirow[t]{2}{*}{ Importance } & \multicolumn{4}{|c|}{ Scenario in which the issue is addressed } \\
\hline & & $\begin{array}{l}\text { Arms and } \\
\text { legs }\end{array}$ & New teeth & $\begin{array}{c}\text { Skin } \\
\text { Valley }\end{array}$ & Mr. Perfect \\
\hline \multicolumn{6}{|l|}{ Regulation } \\
\hline $\begin{array}{l}\text { Clash of regulatory } \\
\text { requirements (versus people's } \\
\text { needs) - clash of regulatory } \\
\text { regimes }\end{array}$ & 7 points & $x$ & $x$ & & $x$ \\
\hline $\begin{array}{l}\text { Data - who owns the personal } \\
\text { information / who stores the } \\
\text { data? How do we control the } \\
\text { storage of data? }\end{array}$ & 2 points & $x$ & & $\mathrm{x}$ & $x$ \\
\hline Privacy and ownership & 1 point & & & & $\mathrm{X}$ \\
\hline $\begin{array}{l}\text { Over-regulation or lack of } \\
\text { regulation }\end{array}$ & 1 point & $x$ & & $\mathrm{x}$ & \\
\hline \multicolumn{6}{|l|}{ Distribution of benefits } \\
\hline $\begin{array}{l}\text { Ethics - distribution of benefits } \\
\text { / need versus nice to have }\end{array}$ & 2 points & $x$ & $\mathrm{x}$ & & $x$ \\
\hline $\begin{array}{l}\text { Boundary between restoration } \\
\text { (medical) and enhancement }\end{array}$ & 1 point & & & $\mathrm{X}$ & $x$ \\
\hline $\begin{array}{l}\text { What would make one lose } \\
\text { entitlement to healthcare? }\end{array}$ & 1 point & & $x$ & & \\
\hline $\begin{array}{l}\text { Ensure balance between social } \\
\text { care and healthcare }\end{array}$ & 1 point & & $x$ & & \\
\hline \multicolumn{6}{|l|}{ Citizen roles } \\
\hline $\begin{array}{l}\text { Promote public dialogue and } \\
\text { consultation on emerging } \\
\text { technologies and their } \\
\text { implication - also to not } \\
\text { oversell and make hype on } \\
\text { potential research results }\end{array}$ & 2 points & & $x$ & & \\
\hline $\begin{array}{l}\text { Responsibility for appropriate } \\
\text { use: education of healthcare / } \\
\text { who is responsible if anything } \\
\text { goes wrong / informed consent }\end{array}$ & 1 point & $x$ & $\mathrm{x}$ & $\mathrm{x}$ & $\mathrm{x}$ \\
\hline $\begin{array}{l}\text { Provide incentives and } \\
\text { measures to support skills and } \\
\text { capacities (training) for 3D } \\
\text { printing technology usage and } \\
\text { promote public discussion on } \\
\text { the implications of this } \\
\text { technology (advantages, risks } \\
\text { etc.) }\end{array}$ & 1 point & $\mathrm{x}$ & & $\mathrm{x}$ & \\
\hline Rethink education & 1 point & & & & \\
\hline \multicolumn{6}{|l|}{ Other } \\
\hline $\begin{array}{l}\text { Policy implication - research } \\
\text { funding policy should not } \\
\text { penalise areas that showed little } \\
\text { "positive" results }\end{array}$ & 1 point & & & & V \\
\hline $\begin{array}{l}\text { Food printed out, I would never } \\
\text { eat it (ban printed food or } \\
\text { oblige to inform public what } \\
\text { food is printed) }\end{array}$ & 1 point & & V & & \\
\hline
\end{tabular}




\section{Analysis of policy issues}

Due to the heterogeneity of bio-AM applications and their emerging status as well as due to the exploratory nature of the project, the present report cannot provide an all-encompassing shared framework for 3D bioprinting which clearly defines the role of the EU, Member States and other policy actors. We thus emphasise, above all, the need for further monitoring of the different application areas in which bio-AM devices are employed or will be employed in the near future.

Nevertheless, in order to be able to develop potentially useful policy options, the following approach is applied: First, in this section, relevant results of the project, and the outcomes of the scenario work in particular, are discussed and taken as the basis for an analysis of policy issues structured along three broad thematic areas: regulation, distribution of benefits and the role of citizens (public participation and citizen-driven innovation). Second, the results of this analysis are used to draft a list of crucial policy challenges at the beginning of section 9. Finally, also in section 9, policy options are presented with a special view to Committees and initiatives of the European Parliament as well as the Work Programme of the European Commission.

One rationale behind choosing this three-step approach is to live up to what Jean-Claude André, in an important and ambitious study on future perspectives of AM (André 2018), recently emphasised by citing a French study on disruptive innovation (Charrié \& Janin 2017), namely that it is crucial to give visibility to entrepreneurs, to on-site actors and to citizens in encompassing pro-innovation policies which are to take place on every level of public decision making and action.

As André (2018) argues, in our view quite rightly so, the future prospects of bio-AM and related fields depend on a crossing of boundaries between science, society, economy and policy (in the broad sense). While this makes foresight exercises extremely complicated, narrower approaches will most probably not help provide society and policy-makers with meaningful orientation regarding the highly heterogeneous applications of bio-AM.

\subsection{Regulation}

3D printing approaches, especially those for biomedical applications, are faced with significant regulatory challenges (Hourd et al. 2015; Gilbert et al. 2017). The complexity and customisation of bioAM artefacts challenge regulation and governance structures.

The need for an appropriate regulation of the new application fields of bio-AM was obvious right from start of the project. It became apparent that the currently established pillars of EU regulation (on such areas as pharmaceuticals, medical devices, advanced therapies, tissues and cells, organs, blood, food and chemicals) would need re-evaluation. Regulation was also the overarching policy issue with most votes in the scenario workshop, more specifically the clash of regulatory requirements (versus people's needs) and the clash of regulatory regimes. In the Envisioning Workshop, it was highlighted that technological development will likely progress regardless of regulatory support, but despite this regulation is an important means for guiding the technological development of regenerative medicine and organ printing in an ethically and societally responsible direction (see section 6).

Another important topic in the scenario work were the differences between EU Member States' national laws and regulations on the actual use and application of AM products, which pose issues of liability (in case of damage or adverse/side effects) and of an international playing field for an emerging industry. Considering the possibilities of distributing the production and use of bio-AM materials and applications across and in various countries and those of the development of a market for bio-AM products, such differences may not only affect innovation but also patient and consumer security. These aspects emerged especially in the Skin Valley and the Arms and Legs for Solidarity scenarios. 
On 5 April 2017, two new European regulations on medical devices were adopted, which will begin to apply after a transitional period ${ }^{119}$; and the effects for bio-AM devices are still unclear. It was highlighted in the horizon scanning work that discussions, also in the European Parliament (EP), about policies for the use of substances of human origin are to be expected in the near future, including the Directive on Tissues and Cells (Directive 2004/23/EC), the Blood Directive (2002/98/EC) and the Regulation on Advanced Therapies (ATMPs, 1394/2007).

Furthermore, regulations and standards from other sectors may apply to the machinery or manufacturing processes, such as the use of lasers and software programmes, thereby further complicating the regulatory landscape. The problem of structural integrity (from a mechanical standpoint) is recognised in upcoming standards, as for example the ISO/TC 261 on Additive Manufacturing - Technical design guideline for powder bed fusion or the related ISO/TC 44/SC14: Additive Manufacturing - System performance and reliability. Standardisation endeavours include various joint ASTM/ISO groups to develop industry best practices for AM, mainly regarding Powder Bed Fusion Applications. The topics Powder, Machine, Digital Product Definition and Data Management as well as the training of personnel are particularly in need of standardisation efforts. Other AM technologies are at this stage not as established in industry, which means that bio-AM applications enabled through Powder Bed Fusion components will have the soonest acceptance in clinics.

As regards the area of tissue engineering, there are also a number of regulatory challenges. Liver replacement is one of the first customised organs which is expected to have high potential, primarily because it is a solid organ for which there is a high demand. Liver replacements would, for example, benefit the thousands of people suffering from alcohol abuse problems. A drawback with these kinds of treatments is that they might reduce incentives to change drinking habits. What is more, if 3D printed organs become available with no regulatory mechanisms, the problem of the black market for organs ${ }^{120}$ may aggravate and a rise in medical tourism may develop. The production of personalised manufactured tissue and organs also calls for an infrastructure for storing the records. Databases on patients for customised organs and tissue are associated with many regulatory issues of data protection and data management, and there is a potential tension between cell/DNA banks and digital databases.

Against the backdrop of the wide variety of relevant regulatory questions, the project specifically looked at a number of regulatory aspects, namely (1) the blurring of boundaries between biomaterial and nonbiomaterial products, (2) ownership of data, intellectual property and privacy, (3) issues of safety and informed consent, and (4) standards and requirements for product development, validation and testing. The results of the project's work on these topics will be presented in the following. It is noteworthy that some of the challenges arising in these contexts are characteristic of the bio-AM application areas or relevant medical areas at large.

\section{Blurring of boundaries between biomaterial and non-biomaterial products}

Many emerging applications of bio-AM are difficult to fit into current legislative pillars or categories. It is unclear whether bio-AM applications are a biological product or a non-biological component, whether they are a medical device or an advanced therapy. This has economic implications for developers as for advanced therapies regulation is more stringent and it is much more complex and costly to get market approval. There is hesitation, however, to compare bio-AM devices to regular medical devices since the interaction with the body is more "natural" during their use. The In Vitro Diagnostic Device Regulation (EU) 2017/746 does not clearly state how substances of human origin

119 https:/ / ec.europa.eu/growth/sectors/medical-devices/regulatory-framework_de (last accessed 19.02.2018).

${ }^{120}$ For example, accroding to the $\mathrm{WHO}$, conservative estimates are that around several thousand black market operations of kidneys take place annually worldwide; see http://www.who.int/bulletin/volumes/85/12/06039370/en/. 
should be judged. The newly revised Medical Device Directive 2017/745 brings some changes; especially the definition of custom-made devices is pivotal in this respect, and how these should be considered under the framework for medical devices. As mentioned above, discussions about policies for the use of substances of human origin are to be expected, also in the EP, with respect to the Directive for Quality and Safety of Human Tissues and Cells (Directive 2004/23/EC), the Blood Directive (2002/98/EC) and the Advanced Therapies Regulation (ATMPs, 1394/2007).

One of the key challenges in regulating AM is acknowledgement of the fact that biological and nonbiological materials are regulated in different ways. AM applications are, however, often considered combination products that include both biological and non-biological components. Examples include combinations of biomaterial scaffolds with human cells or other combinations of synthetic and biological components. Especially when these biological materials are human or human stem cellderived, both ethical concerns and needs for public policy scrutiny tend to increase. There are different regulatory pathways in the EU and US for a 3D printed combination product consisting of a scaffold (medical device) with viable human cells (classified as ATMP in the EU). Comparing regulations between US and EU, there is the question whether current EU regulation is up to date concerning AM innovation, given the fact that in the US fast track approval routes have been developed by the FDA for 3D printed medical devices.

Due to the combination of materials and processes used in AM, current established pillars of EU regulation (pharmaceuticals, medical devices, advanced therapies, tissues and cells, organs) may not be suitable for bio-AM regulation in medicine and thus need re-evaluation.

According to Hourd et al. (2015), little or no scientific guidance is available regarding regulatory pathways for 3D manufacturers in medicine. If the field is to move forward, the R\&D community will need early and regular dialogue with regulatory agencies at particular translational bottlenecks in the manufacturing and quality development process (see Hourd et al. 2015 for a detailed discussion of US and EU regulatory framework for 3D bioprinting in medicine, describing regulation and implications for manufacturers of a 3D printed nasal implant; a summary of the most important points is given in the following). In the EU, a 3D printed tissue or organ can only be regulated as either a medicinal product (drug or biologic, including ATMP) or a medical device. Legislation for these two broad categories differs markedly in terms of procedures to be followed to place the product on the EU market. 3D printed products may combine components and show features of several product categories, which makes classification complex. It is crucial to help manufacturers, e.g. through specific guidance documents, determine the applicable regulatory pathway and identify the product development strategy and risk management plans they need accordingly to bring their product to the market.

For a combination product consisting of cells or tissues and a scaffold matrix, the classification is determined by the way in which the finished product achieves its intended medical purpose, the claimed mode of action (MoA), the characteristics of the active substance and the way in which it is combined in the finished product. For example, for a nasal implant, which is central to one of the scenarios, the conclusion would be the following: On the basis that the product consists of substantially manipulated chondrocyte (cartilage) cells, is presented as having properties for regeneration and repair of a human tissue, and contains (as an integral part) scaffold material that fulfils its function as a medical device when deployed in the patient, the product falls within the definition of a tissue-engineered combined ATMP, as defined in article 2(1)(d) of Regulation (EC) No 1394/2007/EC [15].

Besides the quite complex considerations behind these classifications, in practice the manufacturer may not have sufficient data and/or scientific knowledge in the early development phase to identify the principal MoA and thereby the candidate ATMP classification.

Besides these challenges around classification, regulatory input is furthermore required regarding the following standards in order to clarify requirements for manufacturers: 
- Quality and safety standards for donation, procurement and testing of human cells or tissues under the EU Tissue and Cells Directive (Directives 2004/23/EC, 2006/17/EC and 2006/86/EC),

- standards for the manufacture of products in compliance with the principles of Good Manufacturing Practice (GMP) (Directive 2003/94/EC) and for

- conduct of clinical trials in the EU (Directive 2001/20/EC) in accordance with the principles of Good Clinical Practice (GCP) laid down in Directives 2005/28/ EC and 95/46/EC [21].

Another difficulty is that, in the $\mathrm{EU}$, the regulation requires the device component to receive a separate license (CE mark) in addition to the requirements for the cell-based component. If the device component is an integral part of the ATMP and not manufactured as a separate entity (as in the case example of a nasal implant), this may create a significant regulatory burden for manufacturers. This was the widely perceived view of stakeholders in the European Commission's public consultation on the ATMP Regulation in 2012, in which a single assessment process for combined ATMPs was supported to reduce regulatory uncertainty $[47,48]$.

Regarding the promise of customised products for patients, there seems to be a significant regulatory gap: A clear regulatory definition for an "industrial process" in the field of bio-AM, by which the ATMP regulation distinguishes commercial enterprise from "hospital"-based preparation of therapies, is lacking. This is a problem for distributed manufacturing strategies or patient and hospital exemption (EU) regulatory schemes which try to make individual "batch-of-one" therapies available.

Taken together, according to Hourd (et al. 2015), changes of existing regulations are necessary to bring $3 \mathrm{D}$ printed products to patients on a large scale. Guidance may also be provided by regulations in the US, which are more flexible and less adapted/streamlined than in the EU, which means at least farer reaching implications for the manufacturers.

\section{Ownership of data, intellectual property and privacy}

From the horizon scanning work and expert interviews, it became clear that developments in bio-AM, including data sharing and its use in DIY communities, put current practices of intellectual property rights (IPRs) under pressure and may raise issues of data ownership and privacy.

In our scenarios, the issue of ownership was strongly related to patients' data and materials and covered questions of personal identity (identity of the body with AM devices), ownership of data about health (which are needed to create other devices and thus may foster innovation) as well as use of data to stimulate non-therapeutic applications. It is unclear whether current regulations on medical data and body materials are sufficient for regulating the various applications of bio-AM.

According to some experts (Li 2014), the current patent system is deficient and may create barriers to innovation. The TRIPS agreement (Art 66 TRIPS Agreement; Art 24 UNESCO Universal Declaration on Bioethics and Human Rights 2005) recommends different licensing strategies for different countries in order to grant access to developing countries. The scope of patentability will clearly affect access to future bio-AM therapies, as monopolies may lead to rising prices and innovation barriers for companies not holding relevant patents. So far, Organovo was granted patents for 3D printed tissues for toxicology tests and disease research ( $\mathrm{Li} 2014)$.

In general, a patentable invention can be a product or a process. Potential patenting areas in bio-AM are the derivation and production of extracellular matrix materials (ECMs) and pre-processing of cells that are used for printing as well as methods of dispensing and printing and bioreactor methods that allow the printed tissue or organ to grow to full function after the printing process (Yoo 2015). Patents must be obtained for each relevant national patent office separately ${ }^{121}$, but the EU has the European Patent Office (EPO) as a patent organisation. ${ }^{122}$

121 http://www.wipo.int/portal/en/index.html (last accessed 15.03.2017).

122 https:/ / www.epo.org (last accessed 15.03.2017). 
In the US, the Supreme Court ruled that biological matter is patentable based on the criterion that it is markedly different from the natural one (Ebrahim 2017). It is unclear as to whether a biotechnological product produced by bio-AM technology would also be patentable subject matter, not only in regard to the markedly different criterion but also due to the human organism exception, as the ability to produce living tissues or organs with bio-AM blurs the line between the living and the nonliving (Ebrahim 2017). A definition of "markedly different" would roughly be the fabrication of biological components that do not exist in the natural world, or the redesign of existing biological systems (Ebrahim 2017). As the assembly of cells by means of bio-AM would be combined with artificial materials such as plastic scaffolds, such that a mixed living and nonliving bio-AM product results, this would likely be considered markedly different. For post-processing of materials produced by means of bio-AM, such as a kidney or a heart with vascularisation enabled through artificial materials, the situation is unclear (Ebrahim 2017). Bio-CAD (computer-aided design) files, like any 3D printing CAD file, contain digital blueprint instructions for an object in the form of a digital 3D model (Peacock 2013). Medical images of a biological tissue or organ in an MRI or CT scan can be transformed into CAD files (Chae et al. 2015). It was proposed that the Bio-CAD file should be patentable, and that it should be ruled that a modified Bio-CAD file would have a different structure, a different function or different properties than the natural product on which it is based, and therefore, the printed object would also have different structure, function or properties than the natural object because the modified Bio-CAD file is the equivalent of the printed object (Ebrahim 2017). This would make things clearer for developers and companies and widen patentability. However, it is not clear if this would fasten innovation and increase access.

A practical problem for regulation may be the sharing of CAD files online. Manufacturers of 3D printers or data sharing platforms for CAD files may be sued for making copies possible. It is possible that antiforgery measures become mandatory to be incorporated in low-cost 3D printers, as was done for scanners and printers (Bradshaw et al. 2010). In this case, anti-forgery measures were incorporated to detect attempts to duplicate banknotes. ${ }^{123}$ It is possible that 3D printers could be altered in a way that they can only use authorised CAD files.

Article 5(2) of the Biotech Directive [Directive 98/44/EC of the European Parliament and of the Council of 6 July 1998 on the legal protection of biotechnological inventions (Biotech Directive), Art 5(2)] states that "an element isolated from the human body or produced by means of a technical process may be considered as patentable, even if identical to a natural element". This is probably the most prominent difference to US patent law, as mentioned above. It appears to imply that printed human tissue is patentable, but the so-called morality test still has to be passed (53[a] of the European Patent Convention [EPC]). The morality exclusion applies if human embryonic stem cells are used in bioprinting, based on dignity and identity issues, however not if used for therapeutic purposes (i.e. the so-called medical treatment exemption) (Li 2014).

Medical treatments in contrast to medical inventions (such as pharmaceuticals) are, however, generally not patentable ( $\mathrm{Li}$ 2014). This rule is based on the assumption that medical inventions (pharmaceuticals) are safer if disclosed and patented, but medical treatments patenting will rather restrict patient access. Thus, the implantation of a bioprinted organ or tissue is, like any surgery, not patentable, and in vivo printing neither. The manufacturing process of such a tissue or organ or the product itself would, however, be patentable.

Future IPRs and privacy policies may not only influence the speed of innovation and access to its results but also the structural development of the bio-AM industry in terms of decentralisation vs. centralisation. Investments in technical equipment for bio-AM are already strong. It may be beneficial for innovation and the overall benefits for patients when patenting is still possible, but only for end user

123 T. Thompson, "Security Clampdown on the Home PC Banknote Forgers" (6 June 2004), The

Guardian. https://www.theguardian.com/money/2004/jun/06/crime.uknews (last accessed 15.03.2017). 
products, not for the underlying technologies, as is the case for breeding rights. This approach may ensure that non-patent holders and especially smaller companies can use underlying knowledge to develop and improve bio-AM products. Looking at the current regulation in the EU, it is however questionable if such an approach will be feasible, as only medical treatments such as the implantation of a bioprinted organ or in vivo printing are excluded from patentability. Further complication could be caused by international differences in patent law.

Decentralised production in smaller companies or hospital laboratories is beneficial in terms of speed of delivery. However, extra costs for data protection (both intellectual property and personal data), have to pay out in the end. Another issue regarding safety is that new players such as DIY communities and new producers such as hospitals in a decentralised medical economy raise challenges in terms of oversight of regulations.

The development of AM standards for qualification and certification is complicated due to numerous permutations of machines, materials, processes, and techniques, and the absence of a central depository of AM data and of an authority on AM methodologies. An enhanced deployment of AM technologies will require the development of standards to facilitate quicker and more cost-effective certification of materials, processes and products.

In order to get a better understanding of the implications of patent regimes for the future prospects of bio-AM, both broader and more detailed analyses should be conducted.

\section{Taking measures to establish regulations to handle issues of safety and informed consent}

While benefits of bio-AM for public health are potentially huge, especially when applications become more cost-effective and safe, several authors have warned about risks in terms of safety, in particular of DIY practices (Gilbert et al. 2017; Neely 2016). Several bio-AM applications are entering the clinical trial phase, thereby also creating issues of safety testing in humans for disease modelling or testing of pharmaceuticals (e.g. 3D printed ovaries and real, life-size printed cartilage, bone, ear, muscle tissues, and biocompatible polymers).

We submit that the rise of a consumer market for medical bio-AM applications will become, at least in the long run, an important policy issue as the clinic is not the only space for medical interventions anymore, and new providers of these interventions may not necessarily maintain a healthcare-oriented relation with their customers (including the medical-ethical rules that apply, for example in relation to informed consent, access to care, autonomy, quality and safety standards, protection of vulnerable groups, protection of medical data, clinical effectiveness and good care).

On the other hand, as also the horizon scanning work in this project showed, one should refrain from alarmism in this respect: Even if it would be feasible, for example, to print an organ at home, it would require significant medical expertise and equipment to make it work properly. In the foreseeable future, such very ambitious activities will thus most probably only take place in institutional settings where appropriate regulations are in place.

Other, less ambitious medical endeavours which soon may be successfully pursued in settings with lower safety standards and by (medical) non-professionals are, however, in need of regulation. Here, an instructive example is the DIY body modification community. In this community, which is alternatively called "body hackers", "cyborgs", "DIY transhumanists" or "biohackers"124, medical and non-medical (lifestyle) interventions into the human body are routinely performed in non-medical settings (for example at home or in tattoo studios). While the project did not find any significant evidence of the use of AM technology in this community, this may change soon. In particular in countries where the health systems are not so inclusive as in many European countries, such self-

124 The latter term is, however, more often used for citizen scientists active in molecular biology and genetic engineering. 
treatments or interventions in non-medical settings may become increasingly attractive. A key point here is that the sites matter; a laboratory with controlled conditions and safety standards (GLP) or a production plant with quality assurance practices in place (GMP) differs from development and production at home (Neely 2016).

A related concern in this domain is informed consent, as some patients, for example those on the waiting list for a donor organ or terminally ill, might not be able to make an informed decision when the last hope is a medical intervention in a non-medical setting. Moreover, there might be the risk that the current hype around and fashionable character of "cyborgism" and DIY body modification may attract people who are willing to take unnecessary risks, also with respect to treatments and interventions which are medically not indicated.

While the current relevance of such "experimental" medical and body modification activities should not be exaggerated, it would make sense to already now consider clearly regulating these new areas of medico-techno-scientific practice. When doing this, it is, however, important to take into account that such citizen science - or, for that matter, "citizen medicine" - activities should not only be seen through the lenses of safety concerns. It can be argued that in the course of technological progress they also have the potential to become hotbeds of innovation. As pointed out several times in the present report, it would then be crucial to support skill development among the practitioners, information for the users and the development and oversight of safety standards.

\section{Taking measures to establish standards and requirements for product development, validation and testing}

While the establishment and control of standards may be especially important with respect to new players entering the health domain, there is another challenge raised here concerning the technology itself, no matter in what kind of setting it is used. It remains still unclear whether bio-AM applications can be assessed in a similar way as existing products and services or if a completely new validation and testing framework is needed.

Existing regulatory frameworks do not account, for example, for aspects related to customisation or to differences between products manufactured using 3D printing technology and conventional tissue engineering methods (Hourd et al. 2015). One issue is that complex geometries and microstructures enabled by 3D printing create challenges of biocompatibility and sterility. Even if the selected 3D printing material is clinically approved as part of a market-approved device, the manufacturer needs to establish its compatibility with biological materials and the printing process (Murphy 2014). This requires an understanding of the effect of the bioprinting process on the material as even small changes in the chemical, physical and mechanical properties can affect biocompatibility (Pink 2015).

There are also specific requirements for reproducibly manufacturing a safe product that is sterile and free of unwanted contaminants. Where it is not possible to sterilise the end product, sterility has to be guaranteed in the starting and raw materials, and the cleaning processes applied to the $3 \mathrm{D}$ printing machine and environment in which the bioprinting and any post-processing takes place (Hourd et al. 2015).

A crucial question for product validation and release is how much testing is required when products are both patient-matched (and thus "batches of one") and made potentially on demand (Hourd et al. 2015). Testing of the finished product, especially when it is completed in vivo after being implanted into the patient, is unlikely to be possible. Beyond optimal design of the manufacturing process, more extensive preclinical and in vivo safety testing and other adaptive approaches are thus needed to provide sufficient risk versus benefit information (Hourd et al. 2015).

Furthermore, as mentioned above, there are specific issues of validating the data and software part of the bio-AM process. In terms of image acquisition, the manufacturer will need to verify the accuracy and resolution of the anatomic image, accounting for the effects of image artifacts and the potential 
variation between scanners. There may be significant regulatory issues related to the CAD-CAM step and the use of manual or computer-aided process planning (CAPP) to translate the design information into the process steps and instructions (Hourd et al. 2015).

Taken together, there is considerable regulatory uncertainty for product developers in the EU with respect to (lack of appropriate) standards, regulations and certification which could slow down innovation.

Furthermore, the question of safety testing is also relevant with respect to the controversial topic of animal testing. As it became clear in the horizon scanning, it is doubtful whether bio-AM may significantly contribute to reducing animal testing, a goal pursued by the political agenda of the European Parliament and by several Member States. Bio-AM techniques offer a potential for the substitution of animal experiments, such as the fabrication of tissues in limited sizes to be used for toxicology assays instead of animal models. A cell culture matrix produced by AM allows to study key aspects of the extracellular environment as individual parameters can be precisely tuned, leaving all other parameters the same. Recently, following the EU ban on cosmetic testing, cosmetic companies have begun to show interest in 3D printing. However, current legislation still demands that virtually all innovations in the biomedical sector are tested in animals.

\subsection{Distribution of benefits}

Social justice and issues of fair or equal access are important societal aspects of bio-AM mentioned in literature as well as in the interviews and workshops with stakeholders conducted during the project. Some of these aspects have also regulatory implications, or may have such implications in the future. Nevertheless, it makes sense to analyse them separately from the regulatory issues, because often they are not (yet) so relevant to "straightforward" policy considerations, but already very important as actual or potential topics of broader political and societal opinion-forming and deliberation. Here, policymakers have a crucial role to play, insofar as they can stimulate and enable discussions which are often not so high on the agenda of developers, practitioners and users. Moreover, questions of social justice and access are of fundamental significance for the self-understanding of European societies and the EU at large. AM, and also bio-AM specifically, are cross-sectionally relevant enabling technologies, and therefore a discussion of their implications for society should be an important element of EU endeavours to promote responsible research and innovation (RRI) in Europe and beyond.

During the project, questions of social justice and of fair or equal access to technology were mainly raised with respect to those bio-AM applications which are already widely deemed societally beneficial, such as applications in the fields of prosthetics and orthotics. As our scenario work, and the Arms and Legs for Solidarity scenario in particular (see above), showed, the local, on-site production of low-cost and highly individualised prostheses is an important example of RRI, in particular with respect to midand low-income countries. At the same it became clear in our scenario work that the realisation of the potential benefits depends, at least when it comes to Europe, on a successful regulatory and cultural adaptation of the new technological options.

Roughly speaking, the following main challenges emerged in the course of the project with respect to issues of social justice and of fair or equal access to bio-AM technology.

\section{Increasing costs in the health sector}

Bio-AM may not only affect the use of specific medical therapies or devices, but vice versa the social, cultural and political organisation of the health sectors in EU Member States also affects the future prospects of the field(s) of bio-AM. The challenge here is to assure that innovation paths and regulation result in affordable, accessible and societally responsible bio-AM applications that improve public health. 
Health-related costs are generally increasing in ageing societies, and this is particularly relevant with respect to new therapeutic options such as costly high-tech medical devices and therapeutics with high development costs. In terms of cost reduction, there are great expectations that bio-AM can contribute to rendering the healthcare system more efficient and less costly. However, it must be taken into account that such hopes might be fuelled by the current hype around AM.

We thus recommend that studies and other activities on the problem of the increasing costs in the health sector should regularly take into account the potentials of bio-AM technologies in this respect as well as the barriers to realising these potentials.

\section{Impacts on the labour market}

Another important aspect concerning social justice is the question of whether and to what extent the rise of AM technology in the medical and healthcare sector could cause the loss of jobs. As mentioned above (see section 3.2.2), AM technology could put pressure on the labour market for dental lab technicians. While it would be difficult, given the technological state of the art and the many factors influencing innovation processes in this area, to forecast the impacts of AM technology on employment in the medical sector, this is certainly an important topic to consider with respect to the social aspects of the technology field.

On a more general note and with respect to the future vision of a decentralisation of production through $\mathrm{AM}$, it should also be reflected on potential impacts on the labour markets in developing countries. There is a tangible mid-term or long-term risk that a materialisation of this vision leads to more job creation in the rich countries at the expense of developing countries (Gebler et al. 2014): If a trend emerged to "near-source" products that are currently manufactured in developing countries, cargo and shipping volumes could be reduced - with potential environmental benefits -, but job losses might occur in poorer countries. AM would thus contribute to a relocation of production to consumer countries. If such a shift would be seen as welcome, there would nevertheless be a need for action concerning the bio-AM-related skill development in Europe.

\section{Making a difference concerning the supply with limb prostheses}

In countries that are war-torn or in which large parts of the population live in poverty and without access to the globalised health market for other reasons, a better supply with limb prostheses is obviously crucial. Here, AM technology can be of utmost importance. The Victoria Hand Project (VHP), for example, which is based at the University of Victoria in Canada, has the mission to provide upper limb prostheses to amputees in need, with a focus on developing countries where amputees have limited or no access to prosthetic care. According to the $\mathrm{VHP}^{\prime}$ s website, 3D printing, 3D laser scanning and 3D software are used to fabricate these devices directly within the countries where they are used, and the production networks and the clinical provision network are trained and supported. According to the university website, the project's 3D printed prosthetic hand and socket system has a total cost of approximately \$200 ( $€ 183$ ) for a printed and assembled system (compared to the five-digit $\$$ or $€$ sums that standard and high-end prostheses cost at the globalised health market). Such activities often receive support from universities or can be university-based, but DIY movements are making important contributions as well, including fab labs in the global South such as The House of Natural Fiber's (HONF) FabLab in Yogyakarta in Indonesia (Schaub 2013).

In the course of the project, we learned, thanks to Melike Sahinol, a researcher at the Orient-Institut Istanbul, about an initiative in Turkey named Robotel Türkiye. As we understood, this initiative emerged from a citizen science activity ( 5 dakika) which successfully connected with institutions in Turkey as well as with "Enabling The Future", an international non-profit that provides children with low-cost prosthetic hands, using AM technology. While the website of Robotel Türkiye (http://www.robotel.org/) is only in Turkish, Sahinol provided us with extensive information on this 
initiative during our horizon scanning work. Based on this information, we posit that an innovation partnership which combines citizen-driven, bottom-up activities with support from established institutions can actually make a difference concerning the realisation of the potentials of AM in the area of limb prostheses. Given that the well-being of populations in countries neighbouring the EU is crucial for our common future, we recommend that in EU policy making consideration should be given to supporting lighthouse projects in neighbouring countries where bio-AM technology is used in the health sector.

Moreover, we would like to point out that also in the EU the potentials of AM technology to support the supply with limb prostheses are already realised to a significant extent. In Germany, for example, the company Makea Industries, a start-up which was founded in the context of a fab lab (i.e. a DIY site), has partnered with a globally leading company (Ottobock) in order to establish an open accessible infrastructure for individual and independent product development in the field of prosthetic technology. Under the roof of this "Open Innovation Space", Makea is operating the Fab Lab Berlin. Within this open digital fabrication workshop, Makea claims, private hobbyists, start-ups or other corporates and institutions are invited to learn how to use digital fabrication machines and apply this knowledge under expert supervision to implement their ideas. As far as we can judge from participant observation and some interaction with members of this community, this aim has been achieved to a significant extent. While it is far from clear how policy-makers on the EU level or in Member States can help such innovation partnerships prosper, we submit that they live up to their above-mentioned aim of bringing together entrepreneurs, practitioners and interested citizens (or "citizen scientists") in an innovative and pro-innovative setting.

\section{Blurring of boundaries between therapy and non-therapeutic body modification}

If one looks at the boundaries between therapy and human enhancement or other body modification techniques, AM technologies are only one, albeit an important, element of pertinent technological progress.

As an early STOA study has already pointed out - and we argue that this observation is still valid -, the topic of (non-therapeutic) "human enhancement" (i.e. any modification aimed at improving individual human performance and brought about by science-based or technology-based interventions in the human body) still constitutes, perhaps more than anything else, a societal and cultural phenomenon. While it touches upon a wide variety of broader societal processes, specific desires and fears of both individuals and society at large, and often deep-seated intellectual traditions in Western and, in particular, European history, it is not a technology issue which can easily be handled in policy contexts.

As is the case with many other STEEPED aspects of bio-AM, the aspect of "human enhancement" is all but specific to the field of bio-AM. Nevertheless, progress in bio-AM may render some of the questions discussed in the long-standing and extensive ethical debate on human enhancement more urgent. While we still agree with the above-mentioned earlier STOA study that the topic of human enhancement is not yet a proper policy issue, but an issue in need of broader societal deliberation, more recent developments necessitate a fresh look at the topic: On the one hand, in the current decade we have witnessed the rise of new movements, such as the "cyborg" movement, which actually experiment, usually outside medical settings, with human enhancement technologies. On the other hand, technological progress has made some non-therapeutic body modification interventions feasible.

In this respect, it is important to notice that older discourses on human enhancement in the 1990s and 2000s were focused on the "improvement of human performance". US research and technology policy activities such as the so-called "NBIC initiative" (NBIC stands for nano, bio, info and cogno/neuro) and the ideology of "transhumanism" contributed to focusing the debate on this aspect of performance enhancement (Coenen 2010). Since then, it has become clearer that such a framing of the discussion is misleading. "Human enhancement" in the sense of performance enhancement is actually but one area 
of body modification without therapeutic goals or in non-medical settings (Rembold 2014). As mentioned above, the rise of DIY movements in this area raises new questions with respect to safety and the topic of informed consent; but also questions concerning identity and the blurring boundaries between therapy and lifestyle choices when it comes to so-called "wishful medicine" become more urgent. Medical progress such as the (medically indicated) implantation of a 3D printed skull into a woman's head fuels far-reaching hopes concerning the use of AM for "cosmetic" surgery. While there are cases, such as the use of AM-based products in gender reassignment surgery, which, due their psychological relevance to the patient/user, are medically indicated, other demands for customised body parts may be more controversial.

It must be emphasised again, however, that the questions that can be asked concerning the distribution of benefits with regard to the use of bio-AM in this context are not different to the ones in the general debate on human enhancement. AM only provides new technological options here, but does not substantially change ethical or societal aspects.

While not specific to AM, the blurring of the boundaries between therapeutic and non-therapeutic applications of bio-AM thus raise challenges concerning clinical validation, safety, and bioethical and broader societal questions. Moreover, the debate about human enhancement - in which bio-AM will increasingly be a topic - is closely connected with more futuristic discussions about the prospects of bio-AM and related techno-scientific fields. Our scenario work therefore prominently included the aspects of the blurring of boundaries between therapy and non-therapeutic body modification, especially in the Mr. Perfect scenario and the Skin Valley scenario.

\subsection{Public participation and citizen-driven innovation}

In this project, the role of citizens concerning the current state and future prospects of bio-AM was a major topic. In this respect, we looked at two aspects: On the one hand, we analysed the topic of public dialogue on bio-AM. On the other hand, we asked to what extent citizen-driven innovation actually plays and potentially can play a role in the development of bio-AM.

For quite some time now, so-called "citizen science" activities are funded at the EU level as well as in some Member States (for example Austria, Germany, and Ireland). The European Commission sees citizen science as an important feature of open science, defining "citizen science" as practices through which the public is taking a more active role in science. In the EC's understanding of the concept, it includes both citizens "doing science" (including, for that matter, technology development) and the activities aimed at helping citizens get a better understanding of science (and technology) and influence research and research policy. The latter aspect is also known as "public engagement" with or "public participation" in science, while the former, more recent aspect is strongly connected with the rise of DIY movements and, no matter in what kind of setting, is about citizens without the pertinent scientific expertise engaging in techno-scientific research or development.

While the involvement of citizens in the latter activities can certainly be deemed a new way of public participation in or public engagement with science (and certain DIY and grassroots activities can be of concern for the public), we submit that it makes sense to distinguish between these two aspects. One reason for this is that many citizens obviously want to have a say in the assessment of scientific developments as well as in research policy, but are not interested in "doing science" themselves. Another reason why we think the distinction makes sense is the fact that DIY and grassroots as well as citizen science activities initiated by scientific institutions may go beyond public deliberation of science and technology issues and also make contributions to research and technology development (RTD) and thereby to innovation processes. While "mere" deliberation can of course also be crucial for innovation processes, citizens "doing science" can make direct contributions to technological progress and societal applications of its results.

In both our horizon scanning and our scenario work, the actual and potential role of citizens, and of grassroots and DIY initiatives in particular, was one of the foci. As regards such topics as human 
enhancement and food printing, it became clear that public engagement in imagining their future prospects will be essential, since innovation processes in these areas will be closely interlinked with farreaching questions concerning societal values which cannot be answered by experts or stakeholder representatives alone but need to give a wide variety of voices a say.

At the same time, AM and bio-AM specifically - in particular, as argued above, when it comes to the field of prosthetics - are an area in which citizens "doing science" and technology development, above all in grassroots and DIY settings, can really make a difference.

Of the various challenges we identified in this respect during the project, the following appear to be the most important.

\section{The distribution of responsibility in bio-AM development}

The scenarios highlighted issues of responsibility which cannot be solved through regulation alone. Trust in, as well as enthusiasm for, the technology also depend on the involvement of citizens in the development of bio-AM. For some citizens, improved training in the relevant skills might be important, for others the ability to understand the societal implications of current and future bio-AM.

The potential for distributed and local production of some bio-AM applications also implies an increased responsibility of the users, not only regarding the safety and quality standards in production (such as in the Arms and Legs for Solidarity scenario or in the Skin Valley scenario) but also concerning the individual behaviour (such as in the New Teeth, New Life scenario). Furthermore, the spread of DIY initiatives raises new challenges with respect to RRI which cannot easily be handled with traditional modes of the public communication of science and public participation.

\section{The public role of researchers and stakeholders}

A public dialogue on bio-AM adequate to the chances and challenges raised by this field is also not feasible without a responsible role of professional researchers and stakeholders. Experts we spoke with share the concern that the hype around bio-AM, especially with regard to organ printing, may cause serious harm to the field. Although their ideas on what would be possible in the coming years differed substantially, they all agreed that this application has huge potential. However, the current hype entails refraining from publishing negative results and overselling actual progress. Although one can argue that grand visions are beneficial for an emerging field of science and technology, the experts are afraid that the hype may in the end create public disappointment and thus impede public and, consequently, policy support for the field. Therefore, policy measures could be taken to help stimulate and organise inclusive and publicly visible deliberations on a solid information base, including the questioning of hype mechanisms in academic and business communities.

\section{The future of citizen science}

In light of what has been said above about the two aspects of citizen science, we submit that not only public dialogue and deliberation on bio-AM should be intensified, but also the potentials of citizendriven innovation should be scrutinised more systematically. Taking grassroots initiatives concerning 3D printed limb prostheses and their interactions with academic institutions and industry as an example, it may be asked to what extent - and how - self-organised citizens may contribute to innovation in bio-AM more broadly and new pro-innovation coalitions can be formed between them and companies, academic institutions and other established actors in the innovation system. 


\section{Policy options}

As mentioned above, this final section outlines options for action against the backdrop of crucial policy challenges (9.1.), which in turn are derived from the analyses above and further results of the project. The options for action (9.2.) are also developed with a view to the still rather few recent and current activities at the EU level specifically targeted at bio-AM and to other activities relevant to this field of research and technology development (9.2.1). In this section, we will also specifically look at the role committees and initiatives of the EP play or may play in the future as well as at activities of the EC. Given the particularities of the emerging field of bio-AM, we emphasise again that it is currently not possible to develop a comprehensive and detailed framework of bio-AM policy options. Therefore, much of the following has a preliminary, explorative or tentative character.

What particularities of the bio-AM field are we referring to here? As the project results presented in the preceding sections show, bio-AM, as it was defined for this project, has a very wide range of actual and potential application areas, and the differences between these areas are huge in terms of the current state of the art and future prospects. While some areas are characterised by highly visionary perspectives and very early exploratory approaches concerning future prospects, other areas already show significant progress in RTD or even instances of successful innovation. This is closely related to the fact that also in the very heterogeneous bio- and medical fields AM is a multipurpose, crosscutting enabling technology par excellence. We have thus often decided to outline options for action that focus on the role of bio-AM in broader fields of science, technology or application. As a matter of course, this makes the task of drafting useful policy options more difficult than is the case with options that only deal with bio-AM itself. And such options for action in broader fields necessarily often refer to a rather general level. However, to counter this tendency, we also present a number of quite detailed ideas concerning very specific actual or possible bio-AM RTD activities and uses. Further progress in science, technology and innovation processes will be necessary in order to bridge this gap.

\subsection{Challenges}

As regards EU-level policy challenges raised by bio-AM, it is useful to shortly reflect on the role of the EU level in the innovation policy mix in Europe. As Reillon (2016), in an analysis of the fragmentation of the EU innovation landscape (resulting from the diversity of national and regional policies), points out, the competences of the EU with respect to key policies ("R\&D policy", "Industrial and SME policy", "Education and skills policy", and "Regional and cohesion policy") are relatively strong in RTD policy and regional and cohesion policy, but exist mainly (in the case of RTD policy) or only (in the case of regional and cohesion policy) in terms of funding and promotion activities. In these two areas, regulatory measures on the EU level may only play a role in the case of RTD policy, and only potentially and with low competence of the EU. In the case of industrial and SME policy, Reillon sees some competence also in regulatory respects, but in all three areas ("Fund", "Regulate", "Promote") only potentially and with low competence. With regard to education and skills policy, he states that this policy field is an important area of EU competence concerning promotion activities, but points out that it has no competence at all concerning funding and regulation.

Based on this assessment by Reillon, the results of the project concerning regulatory aspects only have moderate relevance with respect to EU policies and only in the areas of RTD policy and industrial and SME policy. Of particular interest, however, could be funding and promotion measures in the field of RTD policy and with respect to regional and cohesion policy. Likewise, promotion measures with regard to education and skills in bio-AM can be of major importance. 
Furthermore, it is noteworthy that Reillon sees strong EU competence with respect to two "key framework conditions": the "Single Market and Competition" and intellectual property rights (IPRs). In particular the latter is of great importance with respect to the field of bio-AM. Furthermore, Reillon deems the promotion of standards an important part of the EU's role in the innovation policy mix in Europe, and sees at least some competence and potential for action in the promotion of a "culture of innovation".

While there are of course overlaps between the areas - such as the impacts of funding or promotion activities in key policy areas on key framework conditions such as the culture of innovation and IPRs , we find Reillon's assessment useful for the purposes of this project. Aside from the regulatory aspects, his assessment may also be useful with respect to both the aspect of distribution of benefits and the aspects of public participation and citizen-driven innovation. While the EU regulatory competence is low or non-existent with regard to access to high-cost healthcare, it may play a significant role with respect to the key framework condition IPRs, also with a view to open access policies. Moreover, the role of citizens in the innovation system is a key field of action with regard to developing a culture of innovation (Reillon 2016, section 3.7), and there are potentials for action on the EU level and at least some competence. As Reillon emphasises, a particular set of values, norms and patterns of behaviour that stimulate the innovation process nowadays appears to be as important as managing the innovation process itself, in particular with a view to fostering creative thinking, collaboration, initiative, openness, and a positive approach to failure and high trust. This is also perfectly in line with the EC's recent emphasis on citizen science initiatives, which are to be supported based on an open approach that focuses on bottom-up, grassroots initiatives.

The following list of policy challenges should be read against this background. Crucial policy challenges we identified in the project are:

Challenge 1: How can an incoherent, piecemeal adaptation of different regulatory frameworks to ad hoc issues be avoided and a process initiated in which issues fundamental to bio-AM innovation and crosscutting the various regulatory frameworks are addressed? These fundamental issues raised by bioAM include, for example: (a) the blurring of the boundaries between biomaterial and non-biomaterial products; (b) the blurring of boundaries between advanced therapies and medical devices; (c) the creation of patient data and body materials and their transformation into new artefacts (which might not be adequately covered by current regulations of medical data and patient materials); (d) the questions of ownership of data, intellectual property and privacy; (e) issues of safety and informed consent; (f) the need for new standards and requirements for product development, validation and testing. In light of EU competencies in the innovation policy mix in Europe, it seems particularly important in terms of regulation to find the right balance between spreading of innovation through open source policy and protecting ownership through patenting regulation and the right balance between protecting citizens' privacy and ownership rights in regard to personal medical data and bodily material, but also other fundamental issues may be tackled by EU policy measures.

Challenge 2: How can the EU support and promote the application of bio-AM technology for improving public health? How can it be ensured that innovation in this field results in affordable, accessible and responsible applications that improve public health? Given the "competence profile" of the EU in this respect, we would argue that measures, in particular with regard to funding and promotion, are recommendable in all of Reillon's four key policy areas: RTD policy, industrial and SME policy, education and skills policy, and regional and cohesion policy. Moreover, the EU could play a significant role here in fostering a culture of innovation. 
Challenge 3: While the role of citizens is already crucial with respect to various aspects of the first two challenges, fostering both public participation and citizen-driven innovation is an important challenge in itself. In order to illustrate this point, we call to mind that in the European concept of RRI inclusive and responsive approaches with regard to the wide variety of needs, viewpoints, understandings and values of citizens in their various roles are deemed crucial for successful innovation processes and stronger embedding of science and technology in European societies. In our view, it is of utmost importance to notice here that public participation and citizen-driven innovation are two closely related, but still distinct elements or aspects of this policy field. While citizen-driven innovation based on RTD (co-)performed by citizens and civil society grassroots initiatives is an important and in many respects new element of public participation in the shaping of science and technology in society, citizens and civil society organisations also have an - in fact increasingly - important role to play in deliberative public engagement activities. The latter is also particularly relevant with regard to value conflicts, including emerging ones around more visionary topics such as "human enhancement", but also with respect to new practices related to such topics and a wide variety of topics that are already of broad interest to many European citizens, such as animal rights and protection and issues of access to benefits of techno-scientific and medical progress.

\subsection{Options}

With reference to the crucial policy challenges outlined above, we will now present and briefly discuss a number of options for action concerning the field of bio-AM. For this purpose, we will first provide some information on relevant recent and current activities on the EU level (9.2.1) before proceeding with the options for action derived from our project results (9.2.2).

\subsubsection{Selected relevant recent and current activities on the EU level}

In June 2014, a workshop on "Additive Manufacturing in FP7 and Horizon 2020" was organised by the European Commission (Directorate-General Research \& Innovation, Directorate D - Key Enabling Technologies, D2 "Advanced Manufacturing Systems and Biotechnologies"). In 2016, the Executive Agency for Small and Medium-sized Enterprises (EASME) of the European Commission published a report specifically analysing the economic potentials of AM. ${ }^{125}$ So EU-level activities focusing on AM have existed for quite some time now. However, only few of them have specifically addressed bio-AM.

Major completed RTD activities funded by the EU are the FP7 projects BIO-SCAFFOLDS126 (2013-2016), which worked on a new strategy for 3D printing of bone-forming cells, RAPIDOS ${ }^{127}$ (2013-2016) on the rapid prototyping of custom-made bone-forming tissue engineering constructs, and HYDROZONES 128 (2013-2017), addressing the problem of cartilage degeneration also by means of AM technology.

Currently, the EU is, for example, funding the RTD projects Symbionica and CerAMfacturing within the H2020 programme. Symbionica ${ }^{129}$ aims at developing a new technological platform integrating cuttingedge innovations in design and manufacturing in order to deliver better medtech products and services for an improved quality of life. The Symbionica objective is to make the production of smart orthopaedic implants/prosthesis technically feasible and economically sustainable with a level of customisation never seen before, including geometrical and morphological customisation to tailor the implants to

\footnotetext{
125 "Identifying current and future application areas, existing industrial value chains and missing competences in the EU, in the area of additive manufacturing (3D-printing)",

publications.europa.eu/resource/genpub/PUB_EA0116676ENN.1.1

126 https://cordis.europa.eu/project/rcn/108698_en.html

127 https://cordis.europa.eu/project/rcn/108972_en.html

128 https://cordis.europa.eu/project/rcn/106543_en.html

129 https://cordis.europa.eu/project/rcn/198346_en.html
} 
patient interfaces for endo-, exo- and hybrid implants made from multiple materials and functional customisation to adapt the prostheses' dynamic and static behaviour to patients' needs (responsiveness to loads, condition-based drug delivery etc.) across the patient life cycle. CerAMfacturing 130 is developing ceramic and multi-material components to produce personalised medical products using AM. In several case studies, samples for personalised medical products - microsurgical tools, implants and remedies - will be manufactured starting with the patient-specific physical dimensions and ending with components validated under practically relevant conditions. Ceramics is a material group which requires a lot of special know-how and highly experienced staff. For that reason, AM of ceramics is very far from home applications, but ceramic components are indispensable for consumer goods and medical devices. AM therefore opens up new horizons for novel components made of ceramics in a so far "impossible" geometry.

Another ongoing $\mathrm{H} 2020$ project is FAST ${ }^{131}$ in the field of tissue engineering, which aims to integrate a wide variety of "functions" - mechanics, geometry (porosity and shape), biomaterial, bio-active molecules and surface chemical groups - in one AM process. The final objective of the project is to realise a demonstrator of the proposed hybrid AM technology in order to achieve a small pilot production of scaffolds for bone regeneration with the novel smart features to be tested in some in vivo trials.

The European Research Council (ERC) also funds a small number of relevant projects, including the project $3 D$-JOINT ${ }^{132}$, in which bio-AM is used for developing a joint replacement, and the project CELL HYBRIDGE ${ }^{133}$, which aims at engineering constructs where scaffolds work as stem cell delivery systems actively controlling cell quiescence, proliferation and differentiation.

With the H2020 project AM-motion 134 , the EU is also pushing developments at a strategic level. The project aims at increasing Europe's value proposition for AM technologies and capabilities with a strong focus on a rapid market uptake of AM technologies across Europe by connecting and upscaling existing initiatives and efforts. To this end, the project connects industrial lead users with regional activities to develop a strategy and set up the pillars for the efficient implementation of AM by involving a large number of key stakeholders, particularly from industry. Ultimately, this should reinforce the European ecosystem of AM by improving the conditions for large-scale, cross-regional demonstration and market deployment.

The goal of the H2020 project SMART-map ${ }^{135}$ is to define and implement roadmaps for the responsible development of technologies and services in three fields, among them 3D printing in biomedicine. (The other two fields are precision medicine and synthetic biology and not relevant in our context.) The SMART-map project aims to develop a 3D Printing SMART Map as a roadmap to fully exploit the potential of bio-AM technology by involving 3D printing machine producers, software and services developers, advanced materials producers as well as civil society representatives. The project organised two industrial dialogues on the topic, one of which took place in Munich in February 2017136, the other one in Milan in May 2017137. The results of these workshops, which are already partly video documented online, will be fed into the project's 3D Printing SMART Map. The Munich workshop, which focused

\footnotetext{
130 https://cordis.europa.eu/project/rcn/198348_en.html

131 https://cordis.europa.eu/project/rcn/198809_en.html

132 https://cordis.europa.eu/project/rcn/197262_de.html

133 https://cordis.europa.eu/project/rcn/193481_en.html

134 http://www.am-motion.eu/

$135 \mathrm{http}: / /$ projectsmartmap.eu

$136 \mathrm{http}: / /$ projectsmartmap.eu/ind-dialogues/3d-printing-in-the-biomedical-field-munich/

$137 \mathrm{http}: / /$ projectsmartmap.eu/3d-printing-in-the-biomedical-field-milan/
} 
on RRI in 3D printing in biomedicine, has already been analysed in a SMART-map deliverable (Akca Prill et al. 2017). For reasons analysed in detail in the deliverable, the mix of stakeholder groups was not as even as the organisers had hoped for because the large majority of participants were either from industry or public research institutions, and only three participants were representatives of (other) civil society sectors (including one CSO representative). The participants focused on three topics: an RRI platform for 3D printing, an RRI certification tool and a legal check tool. The organisers point out that the RRI concept was deemed rather abstract by the participants, an observation that can often be made in such workshop settings.

Also the STOA project, whose results are presented in this report, has made some impact on ongoing discussions and other activities around bio-AM. The project established a cooperation with the SMARTmap project, for example by involving SMART-map staff in our project's workshop activities. Moreover, Philip Boucher, policy analyst at the European Parliamentary Research Service and in charge of the STOA project, publicly presented results of the project at the conference IN(3D)USTRY From Needs to Solutions, which took place in Barcelona in October 2017.138

In November 2016, the EFDD Group of the EP organised a conference with the title "The 3D Printing Revolution and the Dental Sector: Opportunities, Challenges and Future Applications", which was attended by a wide variety of stakeholders and policy-decision makers. The event was co-hosted by two Members of the European Parliament from two political groups: Dario Tamburrano (EFDD) and Patrizia Toja (S\&D).

The growing interest in the bio-AM field is also testified by the fact that a contribution to a Future and Emerging Technologies (FET) Flagship activity of the European Commission was submitted by a consortium covering the fields of bioprinting, biofabrication and biomanufacturing, as well as robotics, engineering and information technologies. ${ }^{139}$ This contribution, titled HOPE (Human Organ Printing Era), proposed an endeavour which should help transform European healthcare by developing new 3D tissue models and eliminating organ shortages, creating new 3D bioprinting industries, and transforming traditional surgical practice by personalised in situ (at the hospital) robotic bioprinting. The consortium also expressed the hope that in such a major effort synergies with existing and emerging industries could be exploited, for example in cosmetics (in vitro testing models), agriculture (biofabricated leather and food), space exploration (using 3D human living tissue and mini-organs as radiation sentinels) and future defensive capacity of Europe (employing human 3D tissues and organs for testing the effects of biological, chemical and nuclear weapons and development of protective countermeasures).

\subsubsection{Options responding to regulatory challenges}

In our scenario workshop, "clashs of regulations" were ranked as the most important issue. The notion of a "clash" can refer to different aspects in this context, for example the inadequacy of regulations (in terms of societal needs or in light of technological progress) and conflicting regulations (for example when processes of technological convergence transform regulated fields in terms of products, devices and therapies, or with respect to differences between national regulations which can lead to an increase in medical tourism). From an EU policy perspective, harmonisation and standardisation are needed as well as systematic revisions of existing regulations in light of technological progress. As we argued above, an incoherent, piecemeal adaption of different regulatory frameworks to ad hoc issues would be problematic.

138 https://www.youtube.com/watch?v=2h418_n0ag4; https://www.youtube.com/watch?v=j3oIAJcnY-I
139 https://ec.europa.eu/futurium/en/content/hope-human-organ-printing-era 
On the other hand, we also pointed out, with reference to Reillon (2016), that EU competence in the innovation policy mix in Europe is not particularly strong concerning regulatory action, with the exception of IPR regulations. We will thus refrain from going much into details here with respect to desirable regulatory reforms. Nevertheless, we think that some of the results of our project, in particular some stemming from our workshops, are of interest with regard to options for regulatory action, in particular on a general level.

Given the complexity of IPR issues in bio-AM, which will most probably increase due to technological progress and an increase in economic activities, we submit that a most important (and not too costly) option for action is the funding of specialised legal research on the challenges in this area. In line with the RRI approach, such funding should serve to make the best legal expertise available to policy-makers, but this should include expertise on open access solutions and thus not be limited to approaches which aim at increasing patentability options. The main rationales behind this would be (a) to find a right balance between spreading of innovation through open source policy and protecting ownership through patenting regulation, and (b) to establish an IPR regime which equally serves all players in the emerging innovation landscape of bio-AM and European societies at large. While the funding of such research could take place within European Commission programmes, its results could be useful for the work of the EP Committee on Legal Affairs (JURI) and other committees such as the Committee on Industry, Research and Energy (ITRE), the Committee on the Internal Market and Consumer Protection (IMCO) and, possibly, the Committee on Development (DEVE), given that a future ubiquity of certain bio-AM applications might be highly beneficial to the populations in developing countries. As we have pointed out above, the IPR topic is in some respects closely connected with issues of data ownership and privacy. These aspects should therefore be taken into account in the proposed research funding activities. If a strategy for using AM technology in regional development policy (see below) was developed, the Committee on Regional Development (REGI) could also profit from such research.

When it comes to certain specific regulatory actions, the results of our project suggest the following: As regards the practical issue of sharing CAD files online, it might be worthwhile considering making antiforgery measures mandatory for 3D printers, as was done for scanners and printers (Bradshaw et al. 2010). Given that existing regulations on medical treatments are designed to allow for maximum patient access, it may make sense to at least restrict the patentability of manufactured organs and tissues. In both cases, any regulatory decisions should, however, be based on a thorough assessment of the pros and cons in terms of overall societal benefits, i.e. with a view to the balance between spreading of innovation through open source policy and protecting ownership through patenting regulation.

Among the project results on the topic of IPRs described in the preceding sections, a more general regulatory option seems highly relevant: Patenting in the medical area could be limited to end user products and exclude enabling technologies, as is the case for breeding rights. Such an approach could be particularly beneficial to smaller companies, which could then more easily make contributions to innovation processes.

The issue of standardisation with respect to medical devices may become more pressing due to technological advances in this highly interdisciplinary field. Standardisation efforts are already taking place to some extent with regard to AM as an industrial technology. For medical devices, a similar scenario can be envisioned when the component is not implanted but serves, for example, as a visualising tool, surgical aid or an orthosis. However, upon implantation, the component is in contact with human cells. When the component is (1) comprised of a material unique to the manufacturing process (i.e. different from other materials on the market) or (2) unique in its geometry and functionality, standardisation becomes crucial. 
As regards longer-term perspectives, the field of tissue engineering may create new regulatory challenges. When using technologies such as stereolithography, where the manufactured material is different to the raw material, safety issues can arise. A photolithographic formulation is potentially toxic in the raw state and biocompatible in the final state, but a full conversion is needed in order to avoid safety problems. The application of human cells and the need for material biocompatibility require a whole range of standards and rules which do not exist today.

On a more general level, our project results suggest the following two major general options concerning regulatory strategies.

\section{General option A: A comprehensive regulatory framezork}

A comprehensive regulatory framework for all areas affected by bio-AM at a European level would offer several advantages. It could:

- foster the building of a framework for supporting patients and other human subjects receiving bioAM treatments and offer a clear framework for liability;

- help counterbalance (or even avoid) medical tourism towards countries that allow different applications and are less restrictive;

- improve safety of the applications by helping ensure that quality criteria and ethical standards are met;

- contribute to ensuring equal access for users in different countries since it goes beyond national borders.

Possible disadvantages are:

- A comprehensive framework requires much more work and implies a more delicate and possibly lengthy negotiation process among Member States. This could have a slow-down effect on the innovation regime, in particular on health programmes for less favoured groups (see scenarios Arms and Legs and New Teeth, New Life).

- A comprehensive and stricter regulatory framework may result in high costs and a focus on bioAM applications by industry for those who can afford it.

EU policies could work towards such a comprehensive framework, and the JURI and IMCO EP committees could play an important role here. An open question is, however, if EU competencies suffice to realise such a strategy.

\section{General option B: Case-specific adaption of regulations}

Possible advantages of this regulatory strategy are:

- As a comprehensive framework needs time to develop, a case-specific adaption of regulations seems to be an attractive first and fast solution in order not to slow down innovation at an early time in development.

- Regulations can co-evolve with the latest technological advances and their possible impacts, even if they were not foreseen earlier.

- A case-specific adaptation of regulations could respond more rapidly to the challenges due to widespread of distributed/home devices and applications in bio-AM.

- If there are considerable differences in perceptions and acceptance of different bio-AM applications, then case-specific adaptation may promote those applications that people welcome, and restrict more controversial applications. 
Possible disadvantages are:

- The case-specific adaptation of regulations should be taken with caution as it bears the risk of grey areas and of leading to an incoherent, piecemeal approach. The differences in the regulations between European countries that would result from the absence of a comprehensive framework could lead to medical tourism (also outside the EU) and the need to regulate export/import of medical bio-AM products.

- Furthermore, it raises ethical questions of equal access for users from different countries as well as economic issues of unequal opportunities for national economies, e.g. when IPRs are not protected in all countries.

While a comprehensive approach has clear advantages over a case-specific adaptation of regulations, the latter strategy is certainly more easily implementable. If it is chosen, it will become even more important that the wide range of problematic issues is addressed by policy actors. This would require the involvement of a correspondingly large number of EP committees.

\subsubsection{Options responding to the distribution of benefits}

A very important and increasingly urgent problem EU policy is faced with is the question of the distribution of benefits regarding the cost explosion in healthcare due to the ageing of the population in many European countries. As we already pointed out, experts in the field of bio-AM expect important contributions regarding the effectiveness of treatments and medical devices. Moreover, since many expected bio-AM applications promise to broaden the spectrum of cost-efficient therapeutic measures and could be produced locally, there is a huge potential for the healthcare sector, including new commercial opportunities (for SMEs in particular) and incentives for citizens to become active consumers of novel devices and applications. The question of accessibility depends both on the innovation regime chosen and on the modes of reimbursement. While EU competencies regarding the latter are minimal or non-existent, EU-level policy actors, including EP committees such as The Committee on Environment, Public Health and Food Safety (ENVI) and the EC, can play a major role in promoting the use of bio-AM technology in this context and in funding pilot and lighthouse activities. As pointed out above, we also recommend that studies and other activities on the problem of increasing costs in the health sector should regularly take into account the potentials of bio-AM technologies in this respect as well as the barriers to realising these potentials.

As it seems, current EU-funded RTD activities on bio-AM do not yet take RRI aspects sufficiently into account (see 7.2.1). This means that crucial aspects of successful innovation and the need for an inclusive approach concerning the distribution of benefits are often ignored. The EP may consider taking action with regard to this problem. Given the societally disruptive potentials of AM technology, it may also be considered making the field a testbed for publicly funded civil-society research and technological development activities.

As described in some detail above (6.2), several CSOs and academic institutions have started initiatives working for a better supply with limb prostheses in developing countries. Given the increasing number of war-torn countries in the world-regional neighbourhood of the EU, policy-makers on the EU level should seriously consider supporting such initiatives to a significant extent. Obviously, the EP DEVE Committee can play a key enabling role in this regard. Given that the well-being of populations in countries neighbouring the EU is crucial for our common future, such support of lighthouse projects in regions neighbouring the EU could have major beneficial impacts, also for the EU itself. 
Above (6.2), we also pointed out that, again in the AM application area of RTD for limb prostheses, there are examples of desirable new pro-innovation coalitions involving grassroots initiatives from the DIY movements and citizens using the RTD spaces established by representatives of these movements. Such experimental innovation labs are also attractive for industry involvement. While we think that it is far from clear how policy-makers on the EU level or in Member States can effectively help such innovation partnerships prosper in economically strong countries and regions, there might be potentials for highly beneficial policy action in EU Member States with less well-developed health systems. The European Commission could implement such actions, and EP Committees such as ENVI, ITRE and IMCO could play an important role in this respect.

As regards the potential impacts of the spread of bio-AM technologies on the job market in the medical and healthcare sector, tangible recommendations are very hard to make on a general level. Two important options for action are (1) the further funding of socio-economic research in this area, and (2) the stimulation and organisation of long-term monitoring and stakeholder participation processes for selected subareas of the medical and healthcare sector. Given the significant technological progress that has already been made in the dental sector, it would make sense to choose this sector as a testbed for the second option. Building on the activities initiated from within the EP (see 7.2.1) and the interim findings of this project, a permanent multi-stakeholder process including participants from all relevant professions could be organised. The results of such a process could, for example, be of interest to the EP ENVI and ITRE Committees and be funded within the European Commission's RTD funding programmes.

Open source, for example of data such as bioprinting files, is a concept that is already applied in the bioAM field and may be highly beneficial with regard to a fair distribution of benefits as well. However, open source also poses challenges in terms of safety and quality. This is not an issue restricted to bioAM, but bio-AM highlights that the rise of a consumer market for medical applications will become an important policy issue on various levels. The clinic is not the only space for medical interventions anymore, and new groups of stakeholders do not necessarily maintain a healthcare-oriented relationship with their subjects, including the medical-ethical rules that apply, for example in relation to informed consent, access to care, autonomy, quality and safety standards, protection of vulnerable groups, protection of medical data, clinical effectiveness and good care.

A fair distribution of benefits of bio-AM more generally may also be realised by using approaches that combine open source, regulation, patenting and funding. Two major general options are discernible here:

General option A: A mission-oriented innovation policy towards equal access to bio-AM applications

Possible advantages of this regulatory strategy are:

- This would be an RTD funding policy strategy which supports technological projects aimed at accessible bio-AM applications that address specific (chronic) diseases with high social burdens and which neglects fancy innovations for the "happy few".

- Given the numerous possibilities for bio-AM developments, SME policies could support SMEs to develop applications that are fit for local, regional and national markets and specific niches.

- Patenting would be restricted to end user products, but the underlying technologies would not be patentable.

Possible disadvantages are:

- Risks in terms of safety might be rather serious. 
- It still remains unclear whether applications will in fact be available for all on the basis of such a strategy, as in some markets certain bio-AM applications will continue to be too expensive (e.g. in the area of tissue and organ printing).

\section{General option B: Open innovation policy on bio-AM as competitive technology}

Possible advantages of this regulatory strategy are:

- Taking more strongly into account the limits of EU competence in the field of healthcare, this would be a combination of RTD funding and industrial policy aimed at creating a technologically advanced position in bio-AM and a single market for bio-AM applications.

- RTD funding for consortia of universities, university hospitals and firms to develop advanced bioAM applications. Results of these consortia will be published as open access and open data. Safety and costs are the key design criteria for all projects.

- Firms may still use patenting policies to create competitive market positions, but because of open access and open data, monopoly possibilities are restricted.

- Safety is strictly regulated, ensuring that applications once on the single market will be safe in all Member States.

- Implementation within the national and regional healthcare systems is still the responsibility of these authorities, but once the technology is advanced, cost-effective and safe, it will give a strong push to these national systems to adopt bio-AM.

Possible disadvantages are:

- Higher costs may result from harmonisation and safety regulation efforts

- Some overarching European goals might be hard to reach (at least in the near- or mid-term) due to too great a diversity of regional and Member State approaches

\subsubsection{Options responding to public participation and citizen-driven innovation}

To some extent, public participation and citizen-driven innovation activities may also help foster a fair distribution of the benefits of bio-AM. Why this?

In order to respond to this question and to prepare the presentation of options for action, we emphasise again that public participation and citizen-driven innovation are closely related, but distinct elements or aspects of this policy field: While citizen-driven innovation based on RTD (co-)performed by citizens and civil society grassroots initiatives is an important and in many respects new element of public participation in the shaping of science and technology in society, citizens and CSOs still also have an in fact increasingly - important role to play in deliberative public engagement (PE) activities. The latter is also particularly relevant with regard to value conflicts, including emerging ones around such more visionary topics as "human enhancement", but also with respect to new practices concerning such topics and a wide variety of topics which are already of broad interest to many European citizens, such as animal rights and protection and issues of access to benefits of techno-scientific and medical progress.

Public participation and citizen-driven innovation are thus distinct elements of this policy field because they entail significant different practices in different settings and with different degrees of citizen engagement. But they are also distinct aspects of it because (a) citizen-driven innovation activities necessarily go hand in hand with societal reflection on the RTD performed in them and contribute to the societal shaping of science and technology, and (b) public deliberation and dialogue not only contribute to the latter but can also involve hands-on activities and close interaction with researchers and technology developers. 
Public participation activities in the form of dialogue and deliberation are crucial for innovation processes because they can help informing and steering policy and other decision-making processes and contribute to societal reflection on all STEEPED aspects. If they are successfully organised according to the principles of European RRI, in particular with regard to the aspects of inclusiveness and transparent decision making, they are very well suited to support the fair distribution of the benefits of techno-scientific and medical progress and to bring into light societal hopes, fears and needs. While it is important to notice that such activities only very rarely claim to be representative for the society at large and thus only complement and do not replace democratic decision-making processes, they are thus important contributions to societal opinion-forming and reflection processes.

Citizen-driven innovation processes that involve citizen science, or more precisely citizen RTD, and include practices of social innovation increasingly become important in times when the education level in European societies increases, socio-economic and cultural tendencies support "prosumer", technology-savvy and DIY practices, and creativity becomes ever more important also in economic terms. While the notion of "citizen science" encompasses a wide variety of activities, including quite a few conducted in close cooperation with, or even on behalf of, academic and other institutions, RTD by grassroots initiatives - even if small and often not (yet) on the same level in terms of knowledge and know-how as industry or academic RTD - is of particular interest in our context. The new DIY movements are such grassroots initiatives and are characterised by open innovation approaches and a widespread but strongly interconnected network of innovation spaces, such as fab labs, maker spaces and hacker spaces. They also often seek connections with industry, local policy-makers and other societal actors, thereby helping to build new innovation coalitions.

The following presentation of options for action with respect to these two elements of the policy field not only build on the immediate results of the STOA project, including the interactions with external experts and stakeholders, but also on the manifold experiences made by us in EU-funded and national projects and other activities in the areas of public dialogue, public participation research, ethnographic and interview studies on citizen-driven innovation, and stakeholder dialogues. Analogous to sections 7.2.2 and 7.2.3, we speak of two major general options when referring to these fields. It should be noticed, however, that these options are in no way mutually exclusive or alternatives but can be combined.

General option A: Public participation to strengthen public values in the development of bioAM applications

In the STOA project, the scenario workshop participants strongly engaged with the topic of public participation and the question of what role citizens play in innovation processes. In each of the technomoral vignettes, it became clear, through the use of first person stories, that RRI in bio-AM also strongly depends on the roles citizens take, and not only on key players in the innovation system. In the workshop results, this was reflected in the highlighting of the issues of stimulating public dialogue, improved training in 3D skills, and education aimed at ensuring that people can handle the impacts of the new technology field.

When conducting public dialogue and deliberation activities, the goals of these activities should be fully transparent, including later use of the results, in order to allow citizens to make informed decisions whether and to what extent they want to get involved them.

In both our horizon scanning and our scenario work, the actual and potential role of citizens, and of grassroots and DIY initiatives in particular, was one of the foci. As regards such topics as human enhancement and food printing, it became clear that public engagement in imagining their future 
prospects will be essential since innovation processes in these areas will be closely interlinked with farreaching questions concerning societal values which cannot be answered by experts or stakeholder representatives alone but need to give a wide variety of voices a say.

As confirmed by all experts we interacted with, the current hype around bio-AM entails refraining from publishing negative results and overselling actual progress. Although one can argue that grand visions are beneficial for an emerging field of science and technology, the experts fear that the hype may in the end create public disappointment and thus impede public and, consequently, policy support for the field. Therefore, policy measures could be taken to help stimulate and organise inclusive and publicly visible deliberations on a solid information base, including the questioning of hype mechanisms in academic and business communities.

At the same time, public dialogue on all potentials of bio-AM is also needed in order to stimulate reflection on the future of European societies in this context. As long as such very visionary activities do not fuel the hype, they can and should be imaginative, for example also with the help of art. Interfaces between art and science must, however, not be reduced to very visionary aspects. In the STOA project we, for example, had an exchange with such artists as Lale Rodgarkia-Dara who are already involved in art projects using bio-AM technology. Societal issues such as human enhancement, but also technological visions building on 3D bio-AM such as $4 \mathrm{D}$ bioprinting, are legitimate topics of public dialogue, but the EU may aim to find a right balance between more futuristic activities and deliberations in which citizens and CSOs have their say in the shaping of current innovation processes.

Public dialogue and deliberation projects on other multi-purpose enabling technologies, such as nanotechnologies, have shown that a focus on the technology field itself can on the hand lead to discussions that encompass a wide range of STEEPED aspects, but also usually necessitate the selection of a set of application areas to be focused on in the project or event. Another option for action would thus be to promote the systematic inclusion of AM among the topics to be deliberated on in projects or events on broad areas such as health technologies.

General option B: Citizen-driven bio-AM applications to foster creativity, know-how and regional innovation coalitions

As mentioned above with regard to the topic of limb prostheses supply in poorer countries, direct funding and other support of citizen and CSO RTD and innovation initiatives can be an attractive option for action. This is not only due to the tangible societal benefits such funding and supporting measures can have, but also because such initiatives contribute to the increase of scientific and technological knowledge and know-how and their embedding in society. The European Commission is already a very important player in this area and plans to further increase its funding activities on citizen science within H2020.

While the strategy not to predefine top-down areas of RTD in which citizens or CSOs should get engaged is perfectly in line with the RRI principles, an important option for action is a more targeted funding and support of grassroots initiatives in citizen science, and in our context in particular of citizen RTD (citizen technoscience). At the moment, most funding by the European Commission - and the situation is similar in at least a number of Member States - goes to established institutions such as the traditional key players in science communication and universities, which then manage these activities, involving grassroots initiatives only in certain activities and often focusing on established educational or public dialogue modes of science communication or on research on citizen RTD. Consideration could thus be given to incorporating more specific rules into the EC's work programmes concerning how and to what (also financial) extent grassroots initiatives have to be included in EU-funded citizen science 
projects. The cascade funding opportunities provided in H2020 could be particularly useful in this context, and a fair balance between the various actor groups in the projects (academic institutions, companies, civil society grassroots initiatives etc.) is of utmost importance.

Similar options for action exist with respect to research led or entirely conducted by CSOs. The latter kind of citizen involvement in science, technology and the shaping of innovation processes has become increasingly important in recent years.

Forthcoming $\mathrm{H} 2020$ projects ${ }^{140}$ on and in citizen science, which are expected to include a strong element of citizen RTD and citizen-driven innovation, may include grassroots initiatives that use AM technology, at least for medical purposes.

An ambitious European strategy, which may also help to overcome the social bias in EU-funded RRI activities (in which middle/upper class and highly educated citizens appear to be overrepresented), would be a programme designed to foster the creation and maintenance of an EU-wide network of citizen-shaped innovation spaces, with a special view to disadvantaged areas such as (former) workingclass areas with high rates of unemployment or remote areas and economically weaker countries. In many existing initiatives and their spaces, AM technology already plays a central role. However, these initiatives and spaces are usually located in economically strong urban areas, and the large majority of members are young and highly educated citizens. These initiatives and spaces, and startups emerging from them, have in the meantime become highly attractive for companies from a variety of sectors, including the health sector. In the above-mentioned disadvantaged areas, public funding could make a major impact, in particular if the initiatives and spaces are long-term. Research funding and industrial and regional development policies could go hand in hand in such a programme, also helping to counter negative impacts of deindustrialisation processes. And even if the disadvantaged areas and regions did not directly profit from such spaces, a major challenge in the field of AM would be tackled in any case: the improvement of education in this field. This kind of citizen science education - and even sceptics concerning the immediate relevance of citizen RTD agree with this point - can be fostered in such innovation places.

\subsubsection{Summary of key policy options}

A summary of the policy options is presented below, along with the main possible advantages $(+)$, and disadvantages (-) and further options.

\section{Regulation}

\section{- General option 1A: A comprehensive regulatory framework}

+ Counterbalances medical tourism

+ Protects safety through quality and ethical standards

+ Ensures equal access for developing countries

- More time and resource consuming negotiation and development process

- Higher costs for end products

\section{- General option 1B: Case-specific adaptation of regulations}

+ Attractive first and fast solution in order not to slow down innovation in an early phase

+ Regulations can co-evolve with technological advances and respond more rapidly to the distributed/home manufacturing and application

140 https://ec.europa.eu/research/participants/portal/desktop/en/opportunities/h2020/ 
+ May have greater specificity in order to better react different applications of bio-AM

- Risk of grey areas and of an incoherent, piecemeal approach

- Ethical concerns regarding unequal access across the EU due to different IPR

- Additional regulation needed for import/export of bio-AM products

\section{- Further options}

1.1. Funding of specialised legal research on IPR, data ownership and privacy issues in bio-AM could result in a better understanding of the impact of current regulations on innovation and citizen rights, and help to adjust regulation to decrease innovation barriers and improve access

1.2. Anti-forgery measures could be made mandatory in 3D printers in order to prevent illegal sharing of CAD-files online

1.3. Patenting could be limited to end user products (e.g. printed organs and tissues) and patents denied for enabling bio-AM technologies/processes, also in order to give smaller developers the chance to use and improve existing technology. This may also incentivise the development of end user products, and ultimately maximise product availability for patients

\section{Distribution of benefits}

- General option 2A: A mission-oriented innovation policy towards equal access to bio-AM applications

+ Supports the development of bio-AM applications that address diseases with high societal relevance and health care burdens and neglects fancy innovations for the "happy few"

+ SME policies enable the development of applications that are fit for regional to national markets

- Higher safety risks, because production processes are less standardised and regulated

- Applications could still be too expensive, especially in the area of tissue and organ printing

- General option 2B: Open innovation policy on bio-AM as competitive technology

+ Safety is strictly regulated on a single, harmonized EU market

+ Patenting is possible (also for processes), but due to the combination with open access/data policies and RTD funding for consortia of academia and firms, monopolies are still restricted to ensure patient access

- Higher costs may result from harmonisation and safety regulation efforts

- Some overarching European goals might be hard to reach (at least in the near- or mid-term) due to too great a diversity of regional and Member State approaches

- Further options

2.1: Promote the use of bio-AM technology in health care

2.2: Support pilot, lighthouse and grassroots activities, also outside of the EU (for example limb prostheses for people in war-torn countries in the world-regional neighbourhood of the EU)

2.3: Take into account the potentials of bio-AM in studies and other activities on the problem of the increasing costs in the health sector

2.4: Take RRI aspects more into account in EU-funded RTD activities

2.5: Fund socio-economic research and long-term monitoring, including stakeholder participation processes, on the impact of bio-AM implementation on the job market (with the dental sector as a suitable first case study). 


\section{Public participation and citizen-driven innovation}

It is important to notice that the following two general options are not alternatives but can be combined because they each refer to one distinct element of a field of action, not to different strategies concerning the entire field. Accordingly, potential measures are listed for each option (and not, as above, advantages and disadvantages).

- General option 3A: Public participation to strengthen public values in the development of bioAM applications

3.A.1: As regards such topics as human enhancement and food printing, public engagement activities in imagining their future prospects could be promoted and funded in order to early on deal with far-reaching questions concerning societal values in an inclusive manner

3.A.2: As regards the risks of the current and future hypes, measures could be taken to help stimulate and organise inclusive and publicly visible deliberations on a solid information base, including the questioning of hype mechanisms in academic and business communities.

3.A.3: As a multi-purpose enabling technology, bio-AM may profit from public dialogue and deliberation projects and other activities which do not focus on bio-AM technology itself but systematically include AM among the topics to be deliberated on concerning broad areas such as health technologies.

- General option 3B: Citizen-driven bio AM-applications to foster creativity, know-how and regional innovation coalitions

3.B.1: Direct funding (e.g. in H2020 and FP9) and other support of citizen and civil-society-driven RTD and innovation initiatives using AM technology can be an attractive option for action, due not only to the tangible societal benefits such funding and supporting measures can have, but also because such initiatives contribute to the increase of scientific and technological knowledge and know-how and their embedding in society.

3.B.2: In this regard, a more targeted funding and support of grassroots initiatives in citizen science, and in in particular of citizen RTD (citizen technoscience) could be considered, including the incorporation of more specific rules into the EC's work programmes concerning how and to what (also financial) extent grassroots initiatives have to be included in EU-funded citizen science projects (e.g. by means of cascade funding). Similar options for action exist with respect to research led or entirely conducted by CSOs.

3.B.3: An ambitious European strategy, which may also help to overcome the social bias in EUfunded RRI activities (in which middle/upper class and highly educated citizens appear to be overrepresented), would be a programme designed to foster the creation and maintenance of an EU-wide network of citizen-shaped innovation spaces, with a special view to disadvantaged areas where public funding could make a major impact, in particular if the initiatives and spaces are longterm. Research funding and industrial and regional development policies could go hand in hand in such a programme, also helping to counter negative impacts of deindustrialisation processes and to foster education in AM technology.

\subsubsection{Concluding remarks}

As this report and the final sections in particular have shown, there is a gap between current and shortterm societal opportunities, societal risks and policy challenges related to bio-AM on the one hand, and long-term, often highly visionary prospects on the other hand. While the former allow developing a number of tangible options for action, the latter, as long as their assessment is based on the relevant expertise, can be tackled with plausible far-future scenarios. The gap is caused by the lack of mid-term scenarios that are evidence-based and anticipatory in a plausible manner. It might very well be the case 
that in studies such as the present report this gap can never be bridged, at least when it comes to enabling technologies that cross-cut a wide range of science, technology and application areas. While the current state of the art can be rather easily assessed and far-future perspectives can be plausibly dealt with on the basis of obvious potentials of the technologies in question, developments which will take place within the next about five to fifteen years are very hard to foresee due to the contingencies of socio-technical developments. While such mid-term orientation knowledge would be most useful for decision-makers, it is the hardest to generate in a convincing manner.

If these observations are valid, prognostic ambitions concerning mid-term future prospects should be very modest. The main task is then to design inclusive processes for the societal shaping of the technology fields in question. In this respect, the EU has the advantage of having an already welldeveloped, comprehensive approach to responsible innovation, the RRI concept, which puts particular emphasis on inclusiveness and the co-creation of innovation by a wide variety of stakeholder groups, including public participation. As regards the inclusion of a diversity of stakeholders, the STOA project this study reports on was successful. This did not make it an easier but an even more difficult task to generate results useful for policy-makers, precisely due to this diversity which entails a broad range of value positions and interests. Moreover, similar research activities, such as the ones in the abovementioned SMART-map project have shown that the RRI concept is still in need of further development (Akca Prill et al. 2017). Due to its manifold implications for the future of European societies in terms of techno-social progress and its broadly enabling character, the field of bio-AM is very well suited to become another testbed for the RRI approach. This would, however, necessitate a reflection on the current limits of this approach and an adaptation with regard to the current and potential new proinnovation coalitions between policy actors, companies, academic institutions, other established players in the innovation system and citizens who are engaged in citizen-driven innovation initiatives or simply want to have their say concerning the future of science and technology in European societies. 


\section{References}

Abdel-Moniem Barakat, A.; Abou-ElFetouh, A.; Hakam, M. M.; El-Hawary, H. \& Abdel-Ghany, K. M. (2014): »Clinical and radiographic evaluation of a computer-generated guiding device in bilateral sagittal split osteotomies«. Journal of Cranio-Maxillofacial Surgery 42, e195-e203.

Achillas, C.; Aidonis, D.; Iakovou, E.; Thymianidis, M. \& Tzetzis, D. (2015): »A methodological framework for the inclusion of modern additive manufacturing into the production portfolio of a focused factory«. Journal of Manufacturing Systems 37, Part 1, 328-339.

Ahearn, A. (2016): »Ethical Dilemmas in Liver Transplant Organ Allocation: Is it Time for a New Mathematical Model?«. AMA Journal of Ethics 18(2), 126-132.

Ahn, S.-H.; Montero, M.; Odell, D.; Roundy, S. \& Wright, P. K. (2002): »Anisotropic material properties of fused deposition modeling ABS«. Rapid Prototyping Journal 8(4), 248-257.

André, J.-C. (2017): From additive manufacturing to 3D/4D printing. 3: Breakthrough innovations : programmable material, 4D printing and bio-printing. London, UK: ISTE Press.

Anssari Moin, D.; Hassan, B. \& Wismeijer, D. (2016): »A novel approach for custom three-dimensional printing of a zirconia root analogue implant by digital light processing«. Clinical Oral Implants Research.

Arzt, E.; Gorb, S. \& Spolenak, R. (2003): »From micro to nano contacts in biological attachment devices«. Proceedings of the National Academy of Sciences 100, 10603-10606.

ASTM (2010): ASTM F2792-10, Standard Terminology for Additive Manufacturing Technologies. West Conshohocken, PA: ASTM International.

Autumn, K. (2006): »Frictional adhesion«. Journal of Experimental Biology 209, 3569-3579.

Azari, A. \& Nikzad, S. (2009): »The evolution of rapid prototyping in dentistry«. Rapid Prototyping Journal 15, 216-225.

Banks, J. (2013): »Adding Value in Additive Manufacturing«. IEEE Pulse 4, 22-26.

Bártolo, P. J. (ed.) (2011): Stereolithography: Materials, Processes and Applications. Boston, MA: Springer US.

Bendsøe, M. P. \& Sigmund, O. (2004): Topology Optimization. Theory, Methods, and Applications. Berlin, Heidelberg: Springer.

Bentz, R. M. \& Balshi, S. F. (2012): »Complete Oral Rehabilitation With Implants Using CAD/CAM Technology, Stereolithography, and Conoscopic Holography«. Implant Dentistry 21, 8-12.

Berke, G. M.; Fergason, J.; Milani, J. R.; Hattingh, J.; McDowell, M.; Nguyen, V. \& Reiber, G. E. (2010): »Comparison of satisfaction with current prosthetic care in veterans and servicemembers from Vietnam and OIF/OEF conflicts with major traumatic limb loss«. Journal of Rehabilitation Research and Development 47, 361-371.

Berman, M. 1991: Method and apparatus for making three-dimensional objects. (US5071503 A).

Beuer, F.; Schweiger, J. \& Edelhoff, D. (2008): »Digital dentistry«. British Dental Journal 204, 505-511.

Bhise, N. S.; Manoharan, V.; Massa, S.; Tamayol, A.; Ghaderi, M.; Miscuglio, M.; Lang, Q.; Zhang, Y. S.; Shin, S. R.; Calzone, G.; Annabi, N.; Shupe, T. D.; Bishop, C. E.; Atala, A.; Dokmeci, M. R. \& Khademhosseini, A. (2016): »A liver-on-a-chip platform with bioprinted hepatic spheroids«. Biofabrication 8, 14101.

Bidra, A. S.; Taylor, T. D. \& Agar, J. R. (2013): „Computer-aided technology for fabricating complete dentures«. The Journal of Prosthetic Dentistry 109, 361-366.

Bilgin, M. S.; Baytaroğlu, E. N.; Erdem, A. \& Dilber, E. (2016): »A review of computer-aided design/computer-aided manufacture techniques for removable denture fabrication«. European Journal of Dentistry 10, 286-291. 
Boland, T.; Tao, X.; Damon, B. J.; Manley, B.; Kesari, P.; Jalota, S. \& Bhaduri, S. (2007): »Drop-ondemand printing of cells and materials for designer tissue constructs«. Materials Science and Engineering: C 27, 372-376.

Boschetto, A.; Giordano, V. \& Veniali, F. (2013): "Surface roughness prediction in fused deposition modelling by neural networks«. The International Journal of Advanced Manufacturing Technology 67, 2727-2742.

Bourell, D. L.; Watt, T. J.; Leigh, D. K. \& Fulcher, B. (2014): »Performance Limitations in Polymer Laser Sintering «. Physics Procedia 56, 147-156.

British Standards Institute (2012): Publicly Available Specification PAS 83:12 Developing human cells for clinical applications in the European Union and the United States of America - Guide.

Bunzel, B.; Schmidl-Mohl, B.; Grundböck, A. \& Wollenek, G. (1992): »Does changing the heart mean changing personality? A retrospective inquiry on 47 heart transplant patients«. Quality of life research : an international journal of quality of life aspects of treatment, care and rehabilitation 1(4), 251256.

Business of Social Responsibility (BSR): 3-D printing. Sustainability opportunities and challenges (Issue Brief, November 2015).

Chae, M. P.; Rozen, W. M.; McMenamin, P. G.; Findlay, M. W.; Spychal, R. T. \& Hunter-Smith, D. J. (2015): »Emerging Applications of Bedside 3D Printing in Plastic Surgery «. Frontiers in Surgery 2, 25.

Chen, M.; Le, D. Q. S.; Baatrup, A.; Nygaard, J. V.; Hein, S.; Bjerre, L.; Kassem, M.; Zou, X. \& Bünger, C. (2011): »Self-assembled composite matrix in a hierarchical 3-D scaffold for bone tissue engineering «. Acta Biomaterialia 7, 2244-2255.

Chhaya, M. P.; Poh, P. S. P.; Balmayor, E. R.; van Griensven, M.; Schantz, J.-T. \& Hutmacher, D. W. (2015): »Additive manufacturing in biomedical sciences and the need for definitions and norms «. Expert Review of Medical Devices 12, 537-543.

Chin, S. Y.; Poh, Y. C.; Kohler, A.-C.; Compton, J. T.; Hsu, L. L.; Lau, K. M.; Kim, S.; Lee, B. W.; Lee, F. Y. \& Sia, S. K. (2017): »Additive manufacturing of hydrogel-based materials for next-generation implantable medical devices«. Science Robotics 2, eaah6451.

Chitragari, G.; Mahler, D. B.; Sumpio, B. J.; Blume, P. A. \& Sumpio, B. E. (2014): »Prosthetic Options Available for the Diabetic Lower Limb Amputee«. Clinics in Podiatric Medicine and Surgery 31, 173 185.

Chua, C. K.; Leong, K. F. \& Lim, C. S. (2010): Rapid Prototyping. Principles and Applications. Singapore: World Scientific.

Ciocca, L.; Fantini, M.; Crescenzio, F. de; Persiani, F. \& Scotti, R. (2011): »Computer-aided design and manufacturing construction of a surgical template for craniofacial implant positioning to support a definitive nasal prosthesis«. Clinical Oral Implants Research 22(8), 850-856.

Coenen, C. (2009): »Deliberating visions«. In: Kaiser, M.; Kurath, M.; Maasen, S. \& Rehmann-Sutter, Chr. (Hgg.). Governing Future Technologies. Dordrecht, Heidelberg, London, New York: Springer, 73-87.

Commission of the European Communites (2003): COMMISSION DIRECTIVE 2003/94/EC of 8 October 2003 laying down the principles and guidelines of good manufacturing practice in respect of medicinal products for human use and investigational medicinal products for human use. Official Journal of the European Union L 262, 22-26.

Commission of the European Communites (2005): COMMISSION DIRECTIVE 2005/28/EC of 8 April 2005 laying down principles and detailed guidelines for good clinical practice as regards investigational medicinal products for human use, as well as the requirements for authorisation of the manufacturing or importation of such products. Official Journal of the European Union L91, 13-19. 
Commission of the European Communites (2006): COMMISSION DIRECTIVE 2006/17/EC of 8 February 2006 implementing Directive 2004/23/EC of the European Parliament and of the Council as regards certain technical requirements for the donation, procurement and testing of human tissues and cells. Official Journal of the European Union L 38, 40-52.

Commission of the European Communites (2006): COMMISSION DIRECTIVE 2006/86/EC of 24 October 2006 implementing Directive 2004/23/EC of the European Parliament and of the Council as regards traceability requirements, notification of serious adverse reactions and events and certain technical requirements for the coding, processing, preservation, storage and distribution of human tissues and cells. Official Journal of the European Union L 294, 32-50.

Cook, P. S. (2014): »"It's a risk that requires evaluation and rational appraisal": emotion and infectious risk in xenotransplantation«. New Genetics and Society 33(1), 1-20.

Cossins, D. (2012): »Venter supports DNA printers«. https://www.thescientist.com/?articles.view/articleNo/32872/title/Venter-Supports-DNA-Printers/ (accessed: 2017-05-14).

Creylman, V.; Muraru, L.; Pallari, J.; Vertommen, H. \& Peeraer, L. (2013): »Gait assessment during the initial fitting of customized selective laser sintering ankle foot orthoses in subjects with drop foot «. Prosthetics and Orthotics International 37, 132-138.

Crump, S. S. 1992: Apparatus and method for creating three-dimensional objects. (US5121329 A).

d'Aveni, R. (2015): »The 3-D printing revolution«. Harvard Business Review 93(5), 40-48.

Davis, B. K. (2010): »The role of technology in facial prosthetics«. Current Opinion in Otolaryngology $\mathcal{E}$ Head and Neck Surgery 18, 332-340.

de Gans, B.-J.; Duineveld, P. C. \& Schubert, U. S. (2004): »Inkjet Printing of Polymers«. Advanced Materials 16, 203-213.

Deckard, C. R. 1989: Method and apparatus for producing parts by selective sintering. (US4863538 A.).

Derby, B. (2010): »Inkjet Printing of Functional and Structural Materials«. Annual Review of Materials Research 40, 395-414.

Derby, B. (2015): »Additive Manufacture of Ceramics Components by Inkjet Printing«. Engineering $1(1), 113-123$

Despeisse, M.; Baumers, M.; Brown, P.; Charnley, F.; Ford, S. J.; Garmulewicz, A.; Knowles, S.; Minshall, T.H.W.; Mortara, L.; Reed-Tsochas, F. P. \& Rowley, J. (2017): »Unlocking value for a circular economy through 3D printing «. Technological Forecasting and Social Change 115, 75-84.

Dhandayuthapani, B.; Yoshida, Y.; Maekawa, T. \& Kumar, D. S. (2011): »Polymeric Scaffolds in Tissue Engineering Application«. International Journal of Polymer Science 2011, e290602.

Dombroski, C. E.; Balsdon, M. E. R. \& Froats, A. (2014): »The use of a low cost 3D scanning and printing tool in the manufacture of custom-made foot orthoses«. BMC research notes 7, 443.

Domingo-Espin, M.; Borros, S.; Agullo, N.; Garcia-Granada, A.-A. \& Reyes, G. (2014): »Influence of Building Parameters on the Dynamic Mechanical Properties of Polycarbonate Fused Deposition Modeling Parts«. 3D Printing and Additive Manufacturing 1, 70-77.

Dorfinger, P., Stampfl, J., Liska, R. (2015): »Toughening of Photopolymers for Stereolithography (SL)«. In: Edtmaier, C. \& Requena, G. (Hgg.). Materials Science Forum, 53-59.

Drummer, D.; Cifuentes-Cuéllar, S. \& Rietzel, D. (2012): »Suitability of PLA/TCP for fused deposition modelingnull«. Rapid Prototyping Journal 18, 500-507.

Dufner, A. \& Harris, J. (2015): »Trust and altruism--organ distribution scandals: do they provide good reasons to refuse posthumous donation?«. The Journal of medicine and philosophy 40(3), 328-341.

Ebrahim, T. Y. (2017): »3D Bioprinting Patentable Subject Matter Boundaries«. Seattle UL Rev 41, 1. 
Elliott, A. M.; Ivanova, O.; Williams, C. \& Campbell, T. (2013): »An Investigation of the Effects of Quantum Dot Nanoparticles on Photopolymer Resin for Use in Polyjet Direct 3D Printing «. Advanced Engineering Materials 15, 903-907.

Ely, S. (2009): »Personalized medicine«. Translational Research 154(6), 303-308.

Espalin, D.; Alberto Ramirez, J.; Medina, F. \& Wicker, R. (2014): »Multi-material, multi-technology FDM $\ll$. Rapid Prototyping Journal 20(3), 236-244.

European Commission (2014): REPORT FROM THE COMMISSION TO THE EUROPEAN PARLIAMENT AND THE COUNCIL in accordance with Article 25 of Regulation (EC) No 1394/2007 of the European Parliament and of the Council on advanced therapy medicinal products and amending Directive 2001/83/EC and Regulation (EC) No 726/2004 28.03.2014.

European Parliament and the Council of the European Union (1995): DIRECTIVE 95/46/EC OF THE EUROPEAN PARLIAMENT AND OF THE COUNCIL of 24 October 1995 on the protection of individuals with regard to the processing of personal data and on the free movement of such data. Official Journal of the European Union L 281, 31-50.

European Parliament and the Council of the European Union (1998): DIRECTIVE 98/44/EC OF THE EUROPEAN PARLIAMENT AND OF THE COUNCIL of 6 July 1998 on the legal protection of biotechnological inventions. Official Journal of the European Union L 213, 13-21.

European Parliament and the Council of the European Union (1998): DIRECTIVE 98/79/EC OF THE EUROPEAN PARLIAMENT AND OF THE COUNCIL of 27 October 1998 on in vitro diagnostic medical devices. Official Journal of the European Union L 331, 1-37.

European Parliament and the Council of the European Union (2001): DIRECTIVE 2001/20/EC OF THE EUROPEAN PARLIAMENT AND OF THE COUNCIL of 4 April 2001 on the approximation of the laws, regulations and administrative provisions of the Member States relating to the implementation of good clinical practice in the conduct of clinical trials on medicinal products for human use. Official Journal of the European Union L 121, 34-44.

European Parliament and the Council of the European Union (2003): DIRECTIVE 2002/98/EC OF THE EUROPEAN PARLIAMENT AND OF THE COUNCIL of 27 January 2003 setting standards of quality and safety for the collection, testing, processing, storage and distribution of human blood and blood componentsand amending Directive 2001/83/EC. Official Journal of the European Union L 33, 30-40.

European Parliament and the Council of the European Union (2004): DIRECTIVE 2004/23/EC OF THE EUROPEAN PARLIAMENT AND OF THE COUNCIL of 31 March 2004 on setting standards of quality and safety for the donation, procurement, testing, processing, preservation, storage and distribution of human tissues and cells. Official Journal of the European Union L 102, 48-58.

European Parliament and the Council of the European Union (2007): REGULATION (EC) No 1394/2007 OF THE EUROPEAN PARLIAMENT AND OF THE COUNCIL of 13 November 2007 on advanced therapy medicinal products and amending Directive 2001/83/EC and Regulation (EC) No 726/2004. Official Journal of the European Union L 324, 121-137.

European Parliament and the Council of the European Union (2017): REGULATION (EU) 2017/746 OF THE EUROPEAN PARLIAMENT AND OF THE COUNCIL of 5 April 2017 on in vitro diagnostic medical devices and repealing Directive 98/79/EC and Commission Decision 2010/227/EU. Official Journal of the European Union L 117, 176-330.

Faustini, M. C.; Neptune, R. R.; Crawford, R. H. \& Stanhope, S. J. (2008): »Manufacture of Passive Dynamic ankle-foot orthoses using selective laser sintering «. IEEE transactions on bio-medical engineering 55, 784-790.

Fedorovich, N. E.; Wijnberg, H. M.; Dhert, W. J.A. \& Alblas, J. (2011): »Distinct Tissue Formation by Heterogeneous Printing of Osteo- and Endothelial Progenitor Cells«. Tissue Engineering Part A 17, 2113-2121. 
Felzmann, R.; Gruber, S.; Mitteramskogler, G.; Tesavibul, P.; Boccaccini, A. R.; Liska, R. \& Stampfl, J. (2012): »Lithography-Based Additive Manufacturing of Cellular Ceramic Structures«. Advanced Engineering Materials 14(12), 1052-1058.

Feng, F.; Wang, H.; Guan, X.; Tian, W.; Jing, W.; Long, J.; Tang, W. \& Liu, L. (2011): »Mirror imaging and preshaped titanium plates in the treatment of unilateral malar and zygomatic arch fractures«. Oral surgery, oral medicine, oral pathology, oral radiology, and endodontics 112(2), 188-194.

Feng, Z.; Dong, Y.; Zhao, Y.; Bai, S.; Zhou, B.; Bi, Y. \& Wu, G. (2010): »Computer-assisted technique for the design and manufacture of realistic facial prostheses«. British Journal of Oral and Maxillofacial Surgery 48, 105-109.

Ford, S. \& Despeisse, M. (2016): »Additive manufacturing and sustainability«. Journal of Cleaner Production 137, 1573-1587.

Gailey, R.; Allen, K.; Castles, J.; Kucharik, J. \& Roeder, M. (2008): »Review of secondary physical conditions associated with lower-limb amputation and long-term prosthesis use«. Journal of Rehabilitation Research and Development 45, 15-29.

Gebhardt, A. (2007): Generative Fertigungsverfahren. Rapid Prototyping - Rapid Tooling - Rapid Manufacturing. Munich: Hanser.

Gebler, M.; Schoot Uiterkamp, A. J.M. \& Visser, C. (2014): »A global sustainability perspective on 3D printing technologies«. Energy Policy 74, 158-167.

Gerrits, A.; Jones, C. L.; Valero, R. \& Dolinsek, S. (eds.) (2006): A concept of manufacturing system enabling the creation of custom-fit products. 10th Int Research/Expert Conf, Barcelona, Spain, September $11-15$.

Giannatsis, J. \& Dedoussis, V. (2007): »Additive fabrication technologies applied to medicine and health care«. The International Journal of Advanced Manufacturing Technology 40, 116-127.

Gilbert, F.; O'Connell, C. D.; Mladenovska, T. \& Dodds, S. (2018): »Print Me an Organ? Ethical and Regulatory Issues Emerging from 3D Bioprinting in Medicine«. Science and Engineering Ethics 24(1), 1-19.

Goodridge, R. D.; Tuck, C. J. \& Hague, R. J. M. (2012): »Laser sintering of polyamides and other polymers«. Progress in Materials Science 57, 229-267.

Gopalakrishnan, N.; Hannam, R.; Casoni, G. P.; Barriet, D.; Ribe, J. M.; Haug, M. \& Halaas, Ø. (2015): »Infection and immunity on a chip«. Lab Chip 15(6), 1481-1487.

Goyanes, A.; Robles Martinez, P.; Buanz, A.; Basit, A. W. \& Gaisford, S. (2015): »Effect of geometry on drug release from 3D printed tablets«. International Journal of Pharmaceutics 494, 657-663.

Griffiths, C. A.; Howarth, J.; Almeida-Rowbotham, G. de; Rees, A. \& Kerton, R. (2016): ^A design of experiments approach for the optimisation of energy and waste during the production of parts manufactured by 3D printing «. Journal of Cleaner Production 139, 74-85.

Groll, J.; Boland, T.; Blunk, T.; Burdick, J. A.; Cho, D.-W.; Dalton, P. D.; Derby, B.; Forgacs, G.; Li, Q.; Mironov, V. A.; Moroni, L.; Nakamura, M.; Shu, W.; Takeuchi, S.; Vozzi, G.; Woodfield, T. B. F.; Xu, T.; Yoo, J. J. \& Malda, J. (2016): »Biofabrication: reappraising the definition of an evolving field«. Biofabrication 8(1), 13001.

Guo, R.; Lu, S.; Page, J. M.; Merkel, A. R.; Basu, S.; Sterling, J. A. \& Guelcher, S. A. (2015): »Fabrication of 3D Scaffolds with Precisely Controlled Substrate Modulus and Pore Size by Templated-Fused Deposition Modeling to Direct Osteogenic Differentiation«. Advanced healthcare materials 4, 18261832.

Gupta, M. K.; Meng, F.; Johnson, B. N.; Kong, Y. L.; Tian, L.; Yeh, Y.-W.; Masters, N.; Singamaneni, S. \& McAlpine, M. C. (2015): »3D printed programmable release capsules«. Nano Letters 15, 53215329.

Güth, J.-F.; Keul, C.; Stimmelmayr, M.; Beuer, F. \& Edelhoff, D. (2013): »Accuracy of digital models obtained by direct and indirect data capturing «. Clinical Oral Investigations 17, 1201-1208. 
Haddow, G.; Bruce, A.; Calvert, J.; Harmon, S. H.E. \& Marsden, W. (2010): »Not “human" enough to be human but not "animal" enough to be animal - the case of the HFEA, cybrids and xenotransplantation in the UK«. New Genetics and Society 29(1), 3-17.

Hammer, B.; Zizelmann, C. \& Scheufler, K. (2010): „Solid modeling in surgery of the anterior skull base«. Operative Techniques in Otolaryngology-Head and Neck Surgery 21, 96-99.

Hanasono, M. M. \& Skoracki, R. J. (2013): „Computer-assisted design and rapid prototype modeling in microvascular mandible reconstruction«. The Laryngoscope 123(3), 597-604.

Harper, N. G.; Russell, E. M.; Wilken, J. M. \& Neptune, R. R. (2014): »Selective laser sintered versus carbon fiber passive-dynamic ankle-foot orthoses«. Journal of Biomechanical Engineering 136, 91001.

Haske, W.; Chen, V. W.; Hales, J. M.; Dong, W.; Barlow, S.; Marder, S. R. \& Perry, J. W. (2007): »65 nm feature sizes using visible wavelength 3-D multiphoton lithography«. Optics Express 15(6), 3426.

Healthineers, S.: »SOMATOM Force«. SOMATOM Force. https://www.healthcare.siemens.com/computed-tomography/dual-source-ct/somatomforce/technical-specifications (accessed: 2016-11-01).

Heller, C.; Schwentenwein, M.; Russmueller, G.; Varga, F.; Stampfl, J. \& Liska, R. (2009): »Vinyl esters«. Journal of Polymer Science Part A: Polymer Chemistry 47, 6941-6954.

Ho, S. T. B.; Hutmacher, D. W.; Ekaputra, A. K.; Hitendra, D. \& Hui, J. H. (2010): »The evaluation of a biphasic osteochondral implant coupled with an electrospun membrane in a large animal model«. Tissue Engineering. Part A 16, 1123-1141.

Hourd, P.; Medcalf, N.; Segal, J. \& Williams, D. J. (2015): »A 3D bioprinting exemplar of the consequences of the regulatory requirements on customized processes«. Regenerative Medicine 10. (7), 863-883.

Huang, H.-L.; Hsu, J.-T.; Fuh, L.-J.; Lin, D.-J. \& Chen, M. Y. C. (2010): »Biomechanical simulation of various surface roughnesses and geometric designs on an immediately loaded dental implant«. Computers in Biology and Medicine 40, 525-532.

Huang, S. H.; Liu, P.; Mokasdar, A. \& Hou, L. (2013): »Additive manufacturing and its societal impact: a literature review «. The International Journal of Advanced Manufacturing Technology 67(5), 1191-1203.

Huang, W.; Zheng, Q.; Sun, W.; Xu, H. \& Yang, X. (2007): »Levofloxacin implants with predefined microstructure fabricated by three-dimensional printing technique«. International Journal of Pharmaceutics 339, 33-38.

Hull, C. W. 1986: Apparatus for production of three-dimensional objects by stereolithography. (US4575330 A).

Hung, S.-S. \& Lee, Z.-L. (2008): „Clinical application of rapid prototype model in pediatric proximal femoral corrective osteotomy«. Orthopedics 31(1), 72.

Inzana, J. A.; Trombetta, R. P.; Schwarz, E. M.; Kates, S. L. \& Awad, H. A. (2015): »3D printed bioceramics for dual antibiotic delivery to treat implant-associated bone infection«. European Cells and Materials 30, 52.

Izatt, M. T.; Thorpe, P. L. P. J.; Thompson, R. G.; D'Urso, P. S.; Adam, C. J.; Earwaker, J. W. S.; Labrom, R. D. \& Askin, G. N. (2007): »The use of physical biomodelling in complex spinal surgery«. European spine journal : official publication of the European Spine Society, the European Spinal Deformity Society, and the European Section of the Cervical Spine Research Society 16(9), 1507-1518.

Jiménez-Fabián, R. \& Verlinden, O. (2012): »Review of control algorithms for robotic ankle systems in lower-limb orthoses, prostheses, and exoskeletons«. Medical Engineering \& Physics 34, 397-408.

Jin, Y.-a.; Plott, J.; Chen, R.; Wensman, J. \& Shih, A. (2015): »Additive Manufacturing of Custom Orthoses and Prostheses - A Review «. Procedia CIRP 36, 199-204.

Jones, R.; Haufe, P.; Sells, E.; Iravani, P.; Olliver, V.; Palmer, C. \& Bowyer, A. (2011): »RepRap - the replicating rapid prototyper«. Robotica 29, 177-191. 
Joo, H.-S.; Park, S.-W.; Yun, K.-D. \& Lim, H.-P. (2016): „Complete-mouth rehabilitation using a 3D printing technique and the CAD/CAM double scanning method «. The Journal of Prosthetic Dentistry $116,3-7$.

Kaiser, M.; Kurath, M.; Maasen, S. \& Rehmann-Sutter, C. (eds.) (2009): Governing Future Technologies. Dordrecht, Heidelberg, London, New York: Springer.

Karatas, O. H. \& Toy, E. (2014): »Three-dimensional imaging techniques«. European Journal of Dentistry $8,132-140$.

Kataoka, T.; Oka, K.; Miyake, J.; Omori, S.; Tanaka, H. \& Murase, T. (2013): »3-Dimensional Prebent Plate Fixation in Corrective Osteotomy of Malunited Upper Extremity Fractures Using a Real-Sized Plastic Bone Model Prepared by Preoperative Computer Simulation«. The Journal of Hand Surgery 38, 909-919.

Katrina Megget (2016): »How 3D printing could revolutionise medicine«. In: Raconteur (Hg.). https:/ / www.raconteur.net/healthcare/how-3d-printing-could-revolutionise-medicine (accessed: 2016-08-30).

Khaled, S. A.; Burley, J. C.; Alexander, M. R. \& Roberts, C. J. (2014): »Desktop 3D printing of controlled release pharmaceutical bilayer tablets«. International Journal of Pharmaceutics 461, 105-111.

Khaled, S. A.; Burley, J. C.; Alexander, M. R.; Yang, J. \& Roberts, C. J. (2015): »3D printing of tablets containing multiple drugs with defined release profiles«. International Journal of Pharmaceutics 494, 643-650.

Klein, G. T.; Lu, Y. \& Wang, M. Y. (2013): »3D Printing and Neurosurgery - Ready for Prime Time?«. World Neurosurgery 80, 233-235.

Koch, L.; Deiwick, A.; Schlie, S.; Michael, S.; Gruene, M.; Coger, V.; Zychlinski, D.; Schambach, A.; Reimers, K.; Vogt, P. M. \& Chichkov, B. (2012): »Skin tissue generation by laser cell printing«. Biotechnology and Bioengineering 109, 1855-1863.

Kono, K.; Shintani, A.; Okada, H. \& Terada, T. (2013): »Preoperative Simulations of Endovascular Treatment for a Cerebral Aneurysm Using a Patient-Specific Vascular Silicone Model«. Neurologia Medico-Chirurgica 53, 347-351.

Kreiger, M. \& Pearce, J. M. (2013): »Environmental Life Cycle Analysis of Distributed ThreeDimensional Printing and Conventional Manufacturing of Polymer Products«. ACS Sustainable Chemistry \& Engineering 1(12), 1511-1519.

Krini, R.; Ha, C. W.; Prabhakaran, P.; Mard, H. E.; Yang, D.-Y.; Zentel, R. \& Lee, K.-S. (2015): »Photosensitive Functionalized Surface-Modified Quantum Dots for Polymeric Structures via TwoPhoton-Initiated Polymerization Technique«. Macromolecular Rapid Communications 36, 1108-1114.

Kruth, J. P.; Wang, X.; Laoui, T. \& Froyen, L. (2003): »Lasers and materials in selective laser sintering «. Assembly Automation 23, 357-371.

Kruth, J.-P.; Mercelis, P.; van Vaerenbergh, J.; Froyen, L. \& Rombouts, M. (2005): »Binding mechanisms in selective laser sintering and selective laser melting «. Rapid Prototyping Journal 11, 26-36.

Lal, K.; White, G. S.; Morea, D. N. \& Wright, R. F. (2006): »Use of Stereolithographic Templates for Surgical and Prosthodontic Implant Planning and Placement. Part II. A Clinical Report«. Journal of Prosthodontics 15, 117-122.

Langer, R. \& Tirrell, D. A. (2004): »Designing materials for biology and medicine«. Nature 428, 487492.

Leiggener, C.; Messo, E.; Thor, A.; Zeilhofer, H.-F. \& Hirsch, J.-M. (2009): »A selective laser sintering guide for transferring a virtual plan to real time surgery in composite mandibular reconstruction with free fibula osseous flaps«. International journal of oral and maxillofacial surgery 38(2), 187-192. 
Leino, M.; Pekkarinen, J. \& Soukka, R. (2016): »The Role of Laser Additive Manufacturing Methods of Metals in Repair, Refurbishment and Remanufacturing - Enabling Circular Economy«. Physics Procedia 83, 752-760.

Lethaus, B.; Poort, L.; Böckmann, R.; Smeets, R.; Tolba, R. \& Kessler, P. (2012): »Additive manufacturing for microvascular reconstruction of the mandible in 20 patients«. Journal of CranioMaxillofacial Surgery 40, 43-46.

Li, J.; Li, P.; Lu, H.; Shen, L.; Tian, W.; Long, J. \& Tang, W. (2013): »Digital Design and Individually Fabricated Titanium Implants for the Reconstruction of Traumatic Zygomatico-Orbital Defects:«. Journal of Craniofacial Surgery 24, 363-368.

Li, J.; Hsu, Y.; Luo, E.; Khadka, A. \& Hu, J. (2011): „Computer-Aided Design and Manufacturing and Rapid Prototyped Nanoscale Hydroxyapatite/Polyamide (n-HA/PA) Construction for Condylar Defect Caused by Mandibular Angle Ostectomy«. Aesthetic Plastic Surgery 35(4), 636-640.

Li, J.; Ballmer, S. G.; Gillis, E. P.; Fujii, S.; Schmidt, M. J.; Palazzolo, A. M. E.; Lehmann, J. W.; Morehouse, G. F. \& Burke, M. D. (2015): »Synthesis of many different types of organic small molecules using one automated process «. Science 347, 1221-1226.

Li, P. H. (2014): »3D Bioprinting Technologies«. Law, Innovation and Technology 6(2), 282-304.

Li, P. \& Faulkner, A.( 2017): 3D Bioprinting Regulations: a UK/EU Perspective, European Journal of Risk Regulation 8, 441-447.

Lipson, H. (2014): »The Importance of Standards«. 3D Printing and Additive Manufacturing 1, 107.

Liu, Q.; Leu, M. C. \& Schmitt, S. M. (2006): »Rapid prototyping in dentistry«. The International Journal of Advanced Manufacturing Technology 29(3), 317-335.

Lockhande, M. \& Crawford, R. H. (2001): »Testing of compliance in a prosthetic socket fabricated using selective laser sintering «. In: Solid Freeform Fabrication Symposium (Hg.). 2001 Solid Freeform Fabrication Symposium Proceedings, Austin, TX. 2001, 513-526.

Lotz, P. (2006): »The Pardox Of High R\&D And Industry Stability«. Industry and Innovation 5(2), 113137.

Low, S. W.; Ng, Y. J.; Yeo, T. T. \& Chou, N. (2009): »Use of Osteoplug polycaprolactone implants as novel burr-hole covers«. Singapore Medical Journal 50, 777-780.

Lu, S.; Xu, Y. Q.; Lu, W. W.; Ni, G. X.; Li, Y. B.; Shi, J. H.; Li, D. P.; Chen, G. P.; Chen, Y. B. \& Zhang, Y. Z. (2009): »A Novel Patient-Specific Navigational Template for Cervical Pedicle Screw Placement:«. Spine 34, E959-E966.

Mandrycky, C.; Wang, Z.; Kim, K. \& Kim, D.-H. (2016): »3D bioprinting for engineering complex tissues«. Biotechnology advances 34(4), 422-434.

Marga, F.; Jakab, K.; Khatiwala, C.; Shepherd, B.; Dorfman, S.; Hubbard, B.; Colbert, S. \& Gabor, F. (2012): »Toward engineering functional organ modules by additive manufacturing «. Biofabrication 4, 22001.

Martelli, N.; Serrano, C.; van den Brink, H.; Pineau, J.; Prognon, P.; Borget, I. \& El Batti, S. (2016): "Advantages and disadvantages of 3-dimensional printing in surgery «. Surgery 159, 1485-1500.

Materialise Mimics: ». http:/ / biomedical.materialise.com/mimics (accessed: 2016-11-02).

Melchels, F. P. W.; Feijen, J. \& Grijpma, D. W. (2010): »A review on stereolithography and its applications in biomedical engineering «. Biomaterials 31(24), 6121-6130.

Melchels, F. P.W.; Domingos, M. A.N.; Klein, T. J.; Malda, J.; Bartolo, P. J. \& Hutmacher, D. W. (2012): »Additive manufacturing of tissues and organs«. Progress in Polymer Science 37(8), 1079-1104.

Melocchi, A.; Parietti, F.; Loreti, G.; Maroni, A.; Gazzaniga, A. \& Zema, L. (2015): »3D printing by fused deposition modeling (FDM) of a swellable/erodible capsular device for oral pulsatile release of drugs«. Journal of Drug Delivery Science and Technology 30, 360-367. 
Miedzybrodzka, Z. (2003): »Congenital talipes equinovarus (clubfoot)«. Journal of Anatomy 202, 37-42.

Milusheva, S.; Tosheva, E.; Tochev, D. \& Toshev, Y. (2007): »Personalized Ankle Foot Orthosis with Exchangeable Elastic Elements«. Journal of Biomechanics 40, S592.

Mironov, V.; Boland, T.; Trusk, T.; Forgacs, G. \& Markwald, R. R. (2003): »Organ printing«. Trends in Biotechnology 21(4), 157-161.

Mironov, V.; Kasyanov, V. \& Markwald, R. R. (2011): »Organ printing«. Current Opinion in Biotechnology 22, 667-673.

Mohammed, M. I.; Mohan, M.; Das, A.; D. Johnson, M.; Singh Badwal, P.; McLean, D. \& Gibson, I. (2017): »A low carbon footprint approach to the reconstitution of plastics into 3D-printer filament for enhanced waste reduction«. KnE Engineering 2(2), 234-241.

Morineau, T.; Morandi, X.; Le Moëllic, N.; Diabira, S.; Riffaud, L.; Haegelen, C.; Hénaux, P.-L. \& Jannin, P. (2009): »Decision Making During Preoperative Surgical Planning«. Human Factors: The Journal of the Human Factors and Ergonomics Society 51, 67-77.

Mota, C.; Puppi, D.; Chiellini, F. \& Chiellini, E. (2015): »Additive manufacturing techniques for the production of tissue engineering constructs«. Journal of Tissue Engineering and Regenerative Medicine 9, 174-190.

Munoz, S.; van Ramos Jr. \& Dickinson, D. P. (2017): „Comparison of margin discrepancy of complete gold crowns fabricated using printed, milled, and conventional hand-waxed patterns«. The Journal of Prosthetic Dentistry 118(1), 89-94.

Murphy, S. V. \& Atala, A. (2014): »3D bioprinting of tissues and organs«. Nature Biotechnology 32(8), 773-785.

Neely, E. L. (2016): »The Risks of Revolution: Ethical Dilemmas in 3D Printing from a US Perspective«. Science and Engineering Ethics 22(5), 1285-1297.

Ng, P.; Lui, L.; Lee, V. S.P.; Tan, K. C.; Tay, E. H. \& Goh, J. C. (eds.) (2002): Rapid manufacturing machine (RMM) for prosthetic socket fabrication.

Ngan, E. M.; Rebeyka, I. M.; Ross, D. B.; Hirji, M.; Wolfaardt, J. F.; Seelaus, R.; Grosvenor, A. \& Noga, M. L. (2006): »The rapid prototyping of anatomic models in pulmonary atresia«. The Journal of Thoracic and Cardiovascular Surgery 132(2), 264-269.

Nikzad, M.; Masood, S. H. \& Sbarski, I. (2011): »Thermo-mechanical properties of a highly filled polymeric composites for Fused Deposition Modeling «. Materials \& Design 32, 3448-3456.

Ning, F.; Cong, W.; Qiu, J.; Wei, J. \& Wang, S. (2015): »Additive manufacturing of carbon fiber reinforced thermoplastic composites using fused deposition modeling«. Composites Part B: Engineering 80, 369-378.

Norman, J.; Madurawe, R. D.; Moore, C. M.V.; Khan, M. A. \& Khairuzzaman, A. (2017): »A new chapter in pharmaceutical manufacturing: 3D-printed drug products«. Editor's Collection 2016Editor's Collection 108, 39-50.

Örtorp, A.; Jönsson, D.; Mouhsen, A. \& Vult von Steyern, P. (2011): »The fit of cobalt-chromium threeunit fixed dental prostheses fabricated with four different techniques«. Dental Materials: Official Publication of the Academy of Dental Materials 27, 356-363.

Ottnad, T., Kagerer, M., Irlinger, F., Lueth, T.C. (July 2012): »Modification and further development of a drop on demand printhead for wax enabling future 3D-printing and rapid prototyping «. 2012 IEEE/ASME International Conference on Advanced Intelligent Mechatronics (AIM), 117-122.

Pallari, J. H. P.; Dalgarno, K. W. \& Woodburn, J. (2010): »Mass Customization of Foot Orthoses for Rheumatoid Arthritis Using Selective Laser Sintering «. IEEE Transactions on Biomedical Engineering 57, 1750-1756.

Peacock, S. R. (2013): »Why manufacturing matters«. Wm. E Mary L. Rev. 55, 1933. 
Pérez, C. J. L. (2002): »Analysis of the surface roughness and dimensional accuracy capability of fused deposition modelling processes«. International Journal of Production Research 40, 2865-2881.

Pink, J. (2015): »Risks of Additive Manufacturing: a Product Safety Perspective«. https://www.mddionline.com/risks-additive-manufacturing-product-safety-perspective (accessed: 2018-02-09).

Pridoehl, M.; Schmitt, G.; Poppe, D.; Kohlstruk, S.; Hammann, B.; Cremer, S.; Beks, K. \& Dewaelheyns, L. 2014: Multicoloured fused deposition modelling print. (US20140088751 A1).

Quante, K.; Ludwig, K. \& Kern, M. (2008): »Marginal and internal fit of metal-ceramic crowns fabricated with a new laser melting technology «. Dental Materials: Official Publication of the Academy of Dental Materials 24, 1311-1315.

Rai, B.; Lin, J. L.; Lim, Z. X. H.; Guldberg, R. E.; Hutmacher, D. W. \& Cool, S. M. (2010): »Differences between in vitro viability and differentiation and in vivo bone-forming efficacy of human mesenchymal stem cells cultured on PCL-TCP scaffolds«. Biomaterials 31, 7960-7970.

Raman, R.; Cvetkovic, C. \& Bashir, R. (2017): »A modular approach to the design, fabrication, and characterization of muscle-powered biological machines«. Nature Protocols 12(3), 519-533.

Ramu, D.; Ramesh, R. S.; Manjunath, S.; Shivakumar; Goel, V.; Hemnath, G. N. \& Alexander, A. (2015): »Pattern of External Breast Prosthesis Use by Post Mastectomy Breast Cancer Patients in India«. Indian Journal of Surgical Oncology 6, 374-377.

Reddy, J. N. (1993): An introduction to the finite element method: McGraw-Hill: New York.

Rembold, S. (2014): »'Human Enhancement'?«. NanoEthics 8(3), 307-315.

Rengier, F.; Mehndiratta, A.; Tengg-Kobligk, H. v.; Zechmann, C. M.; Unterhinninghofen, R.; Kauczor, H.-U. \& Giesel, F. L. (2010): »3D printing based on imaging data«. International Journal of Computer Assisted Radiology and Surgery 5, 335-341.

Rogers, B.; Gitter, A.; Bosker, G.; Faustini, M.; Lokhande, M. \& Crawford, R. (2001): »Clinical evaluation of prosthetic sockets manufactured by selective laser sintering«. 12th Freeform Fabrication Symposium, Austin, TX, b9.

Rogers, B.; Bosker, G. W.; Crawford, R. H.; Faustini, M. C.; Neptune, R. R.; Walden, G. \& Gitter, A. J. (2009): »Advanced trans-tibial socket fabrication using selective laser sintering «. Prosthetics and Orthotics International.

Rogers, W. E.; Crawford, R. H.; Beaman, J. J. \& Walsh, N. E. (1991): »Fabrication of prosthetic sockets by selective laser sintering «. 1991 Solid Freeform Fabrication Symposium Proceedings, Marcus, HL, Beaman, JJ, Barlow, JW, Bourell, DL, and Crawford, RH, eds., Austin, TX.

Rovick, J. S.; Chan, R. B.; van Vorhis, R. L. \& Childress, D. S. (1992): „Computer-aided manufacturing in prosthetics: Various possibilities using industrial equipment«. Proceedings of the 7 th World Congress of the International Society for Prosthetics and Orthotics. Various possibilities using industrial equipment, 22.

Rovick, J. S.; Childress, D. S. \& Chan, R. (1996): »An additive fabrication technique for the CAM of prosthetic sockets«. Journal of Rehabilitation Research and Development 33, 1.

Rowe, C. W.; Katstra, W. E.; Palazzolo, R. D.; Giritlioglu, B.; Teung, P. \& Cima, M. J. (2000): »Multimechanism oral dosage forms fabricated by three dimensional printing «. Journal of Controlled Release: Official Journal of the Controlled Release Society 66, 11-17.

Sachs, E. M.; Haggerty, J. S.; Cima, M. J. \& Williams, P. A. 1993: Three-dimensional printing techniques. (US5204055 A).

Sadan, A.; Blatz, M. B. \& Lang, B. (2005): »Clinical considerations for densely sintered alumina and zirconia restorations«. The International Journal of Periodontics \& Restorative Dentistry 25, 213-219. 
Salmi, M.; Paloheimo, K.-S.; Tuomi, J.; Wolff, J. \& Mäkitie, A. (2013): »Accuracy of medical models made by additive manufacturing (rapid manufacturing)«. Journal of cranio-maxillo-facial surgery : official publication of the European Association for Cranio-Maxillo-Facial Surgery 41 (7), 603-609.

Sandström, C. G. (2016): »The non-disruptive emergence of an ecosystem for 3D Printing - Insights from the hearing aid industry's transition 1989-2008«. Technological Forecasting and Social Change $102,160-168$.

Sanner, M. A. (2001): »People's feelings and ideas about receiving transplants of different origins questions of life and death, identity, and nature's border«. Clinical Transplantation 15(1), 19-27.

Schantz, J.-T.; Lim, T.-C.; Ning, C.; Teoh, S. H.; Tan, K. C.; Wang, S. C. \& Hutmacher, D. W. (2006): »Cranioplasty after Trephination using a Novel Biodegradable Burr Hole Cover«. Operative Neurosurgery 58, ONS-E176.

Scheper-Hughes, N. (2000): »The Global Traffic in Human Organs«. Current Anthropology 41(2), 191224.

Scheper-Hughes, N. (2003): »Keeping an eye on the global traffic in human organs«. Lancet 361(9369), 1645-1648.

Schievano, S.; Migliavacca, F.; Coats, L.; Khambadkone, S.; Carminati, M.; Wilson, N.; Deanfield, J. E.; Bonhoeffer, P. \& Taylor, A. M. (2007): »Percutaneous pulmonary valve implantation based on rapid prototyping of right ventricular outflow tract and pulmonary trunk from MR data«. Radiology 242(2), 490-497.

Schneider, C.; Lehmann, J.; van Osch, Gerjo J. V. M.; Hildner, F.; Teuschl, A.; Monforte, X.; Miosga, D.; Heimel, P.; Priglinger, E.; Redl, H.; Wolbank, S. \& Nürnberger, S. (2016): „Systematic Comparison of Protocols for the Preparation of Human Articular Cartilage for Use as Scaffold Material in Cartilage Tissue Engineering «. Tissue engineering. Part C, Methods 22, 1095-1107.

Schweizer, A.; Fürnstahl, P. \& Nagy, L. (2013): »Three-dimensional correction of distal radius intraarticular malunions using patient-specific drill guides«. The Journal of Hand Surgery 38(12), 2339_ 2347.

Sengeh, D. M. \& Herr, H. (2013): »A Variable-Impedance Prosthetic Socket for a Transtibial Amputee Designed from Magnetic Resonance Imaging Data«. JPO Journal of Prosthetics and Orthotics 25, 129137.

Silva, N. R.F.A.; Witek, L.; Coelho, P. G.; van Thompson, P.; Rekow, E. D. \& Smay, J. (2011): »Additive CAD/CAM Process for Dental Prostheses«. Journal of Prosthodontics 20, 93-96.

Skardal, A.; Zhang, J. \& Prestwich, G. D. (2010): »Bioprinting vessel-like constructs using hyaluronan hydrogels crosslinked with tetrahedral polyethylene glycol tetracrylates«. Biomaterials 31, 61736181.

Solid Freeform Fabrication Symposium (ed.) (2001): 2001 Solid Freeform Fabrication Symposium Proceedings, Austin, TX.

Song, B.; Dong, S.; Liao, H. \& Coddet, C. (2011): »Process parameter selection for selective laser melting of Ti6Al4V based on temperature distribution simulation and experimental sintering «. The International Journal of Advanced Manufacturing Technology 61, 967-974.

Sood, A. K.; Ohdar, R. K. \& Mahapatra, S. S. (2010): »Parametric appraisal of mechanical property of fused deposition modelling processed parts«. Materials \& Design 31, 287-295.

SPRITAM(R) (SPREE-tam) (levetiracetam) (2016): »Medication Guide 3879800, I. D.« (accessed: 201608-15).

Srivatsan, T. S. \& Sudarshan, T. S. (2015): Additive Manufacturing. Innovations, Advances, and Applications. London: CRC Press.

Stampfl, J.; Baudis, S.; Heller, C.; Liska, R.; Neumeister, A.; Kling, R.; Ostendorf, A. \& Spitzbart, M. (2008): »Photopolymers with tunable mechanical properties processed by laser-based highresolution stereolithography«. Journal of Micromechanics and Microengineering 18, 125014. 
Stampfl, J. \& Liska, R. (2011): »Polymerizable Hydrogels for Rapid Prototyping«. In: Bártolo, P. J. (Hg.). Stereolithography: Materials, Processes and Applications. Boston, MA: Springer, 161-182.

STOA (2009): Human enhancement (IP/A/STOA/FWC/2005-28/SC32 \& 39). European Parliament.

Stoetzer, M.; Rana, M.; See, C. von; Eckardt, A. M. \& Gellrich, N.-C. (2011): »Reconstruction of defects of maxillary sinus wall after removal of a huge odontogenic lesion using prebended 3D titaniummesh and CAD/CAM technique«. Head \& Face Medicine 7, 21.

Stratasys, Inc. Eden Prairie, MN: »FDM - Reviews \& Brand Information«. http:/ / www.trademarkia.com/fdm-74133656.html (accessed: 2015-09-02).

Strub, J. R.; Rekow, E. D. \& Witkowski, S. (2006): »Computer-aided design and fabrication of dental restorations«. The Journal of the American Dental Association 137, 1289-1296.

Sun, J. \& Zhang, F.-Q. (2012): »The Application of Rapid Prototyping in Prosthodontics«. Journal of Prosthodontics 21, 641-644.

Sun, Y. \& Soh, S. (2015): »Printing tablets with fully customizable release profiles for personalized medicine«. Advanced Materials 27, 7847-7853.

Tara, M. A.; Eschbach, S.; Bohlsen, F. \& Kern, M. (2011): »Clinical outcome of metal-ceramic crowns fabricated with laser-sintering technology«. International Journal of Prosthodontics 24(1): 46-48.

Telfer, S.; Gibson, K. S.; Hennessy, K.; Steultjens, M. P. \& Woodburn, J. (2012): »Computer-aided design of customized foot orthoses«. Archives of Physical Medicine and Rehabilitation 93, 863-870.

Telfer, S.; Munguia, J.; Pallari, J.; Dalgarno, K.; Steultjens, M. \& Woodburn, J. (2014): »Personalized foot orthoses with embedded temperature sensing «. Medical Engineering \& Physics 36, 9-15.

Teo, E. Y.; Ong, S.-Y.; Khoon Chong, M. S.; Zhang, Z.; Lu, J.; Moochhala, S.; Ho, B. \& Teoh, S.-H. (2011): »Polycaprolactone-based fused deposition modeled mesh for delivery of antibacterial agents to infected wounds«. Biomaterials 32, 279-287.

Torabi, K.; Farjood, E. \& Hamedani, S. (2014): »Rapid prototyping technologies and their applications in prosthodontics, a review of literature«. Journal of Dentistry, Shiraz University of Medical Sciences 1, $1-9$.

Torgersen, J.; Qin, X.-H.; Li, Z.; Ovsianikov, A.; Liska, R. \& Stampfl, J. (2013): »Hydrogels for TwoPhoton Polymerization«. Advanced Functional Materials 23, 4542-4554.

Traini, T.; Mangano, C.; Sammons, R. L.; Mangano, F.; Macchi, A. \& Piattelli, A. (2008): »Direct laser metal sintering as a new approach to fabrication of an isoelastic functionally graded material for manufacture of porous titanium dental implants«. Dental Materials 24, 1525-1533.

Udoekwere, U. I.; Oza, C. S. \& Giszter, S. F. (2014): »A pelvic implant orthosis in rodents, for spinal cord injury rehabilitation, and for brain machine interface research «. Journal of Neuroscience Methods 222, 199-206.

Vakili, H.; Kolakovic, R.; Genina, N.; Marmion, M.; Salo, H.; Ihalainen, P.; Peltonen, J. \& Sandler, N. (2015): »Hyperspectral imaging in quality control of inkjet printed personalised dosage forms «. International Journal of Pharmaceutics 483, 244-249.

Van Berneveld, J. \& Jansson (2017): Additive manufacturing: A layered revolution. Dublin: Eurofound (European Foundation for the Improvement of Living and Working Conditions).

van Noort, R. (2012): »The future of dental devices is digital«. Dental Materials 28, 3-12.

van Vlierberghe, S.; Dubruel, P. \& Schacht, E. (2011): »Biopolymer-Based Hydrogels As Scaffolds for Tissue Engineering Applications«. Biomacromolecules 12, 1387-1408.

van Woensel, L. \& Vrščaj, D. (2015): Towards scientific foresight in the European Parliament. In-depth analysis. European Parliament.

Vandenbroucke, B. \& Kruth, J.-P. (2007): »Selective laser melting of biocompatible metals for rapid manufacturing of medical parts«. Rapid Prototyping Journal 13, 196-203. 
Vecsei, B.; Joós-Kovács, G.; Borbély, J. \& Hermann, P. (2016): »Comparison of the accuracy of direct and indirect three-dimensional digitizing processes for CAD/CAM systems - An in vitro study «. Journal of Prosthodontic Research 61(2), 177-184.

Ventola, C. L. (2014): »Medical Applications for 3D Printing«. Pharmacy and Therapeutics 39, 704-711.

Vermeulen, N.; Haddow, G.; Seymour, T.; Faulkner-Jones, A. \& Shu, W. (2017): »3D bioprint me: a socioethical view of bioprinting human organs and tissues«. Journal of Medical Ethics 43, 618-624.

Vijayaragavan, E.; Kurian, L. M.; Sulayman, H. \& Gopal, T. V. (2014): »Application of Rapid Prototyping in the Treatment of Clubfoot in Children«. Procedia Engineering 97, 2298-2305.

Walter-Herrmann, J. \& Büching, C. (eds.) (2013): FabLab: Of machines, makers and inventors. Bielefeld: transcript.

Wang, G.; Li, J.; Khadka, A.; Hsu, Y.; Li, W. \& Hu, J. (2012): »CAD/CAM and rapid prototyped titanium for reconstruction of ramus defect and condylar fracture caused by mandibular reduction«. Oral Surgery, Oral Medicine, Oral Pathology and Oral Radiology 113, 356-361.

Wang, X.; Xu, S.; Zhou, S.; Xu, W.; Leary, M.; Choong, P.; Qian, M.; Brandt, M. \& Xie, Y. M. (2016): »Topological design and additive manufacturing of porous metals for bone scaffolds and orthopaedic implants«. Biomaterials 83, 127-141.

Wavreille, O.; François Fiquet, C.; Abdelwahab, O.; Laumonier, E.; Wolber, A.; Guerreschi, P. \& Pellerin, P. (2013): »Surgical and prosthetic treatment for microphthalmia syndromes«. British Journal of Oral and Maxillofacial Surgery 51, e17-e21.

Webb, P. A. (2000): »A review of rapid prototyping (RP) techniques in the medical and biomedical sector«. Journal of Medical Engineering \& Technology 24, 149-153.

Weisman, J. A.; Nicholson, J. C.; Tappa, K.; Jammalamadaka, U.; Wilson, C. G. \& Mills, D. K. (2015): »Antibiotic and chemotherapeutic enhanced three-dimensional printer filaments and constructs for biomedical applications«. International Journal of Nanomedicine 10, 357.

Wentink, M.; Stassen, L. P. S.; Alwayn, I.; Hosman, R. J. a. W. \& Stassen, H. G. (2003): »Rasmussen's model of human behavior in laparoscopy training «. Surgical Endoscopy and Other Interventional Techniques 17, 1241-1246.

Williams, R. J.; Bibb, R.; Eggbeer, D. \& Collis, J. (2006): »Use of CAD/CAM technology to fabricate a removable partial denture framework«. The Journal of Prosthetic Dentistry 96, 96-99.

Wohlers, T. T.; Campbell, R. I. \& Caffrey, T. (2016): Wohlers report 2016. 3D printing and additive manufacturing state of the industry : annual worldwide progress report. Fort Collins, CO: Wohlers Associates.

Wolbring, G.; Martin, A.; Tynedal, J.; Ball, N. \& Yumakulov, S. (2015): »Exploring discourse surrounding therapeutic enhancement of veterans and soldiers with injuries«. Work 50(1), 149-160.

Wu, B. M.; Borland, S. W.; Giordano, R. A.; Cima, L. G.; Sachs, E. M. \& Cima, M. J. (1996): »Solid freeform fabrication of drug delivery devices«. Journal of Controlled Release 40, 77-87.

Wu, M.; Tinschert, J.; Augthun, M.; Wagner, I.; Schädlich-Stubenrauch, J.; Sahm, P. R. \& Spiekermann, H. (2001): »Application of laser measuring, numerical simulation and rapid prototyping to titanium dental castings«. Dental Materials 17, 102-108.

Wurm, G.; Lehner, M.; Tomancok, B.; Kleiser, R. \& Nussbaumer, K. (2011): „Cerebrovascular Biomodelling for Aneurysm Surgery«. Surgical Innovation, 18(3), 294-306.

Xing, J.-F.; Zheng, M.-L. \& Duan, X.-M. (2015): »Two-photon polymerization microfabrication of hydrogels« Chemical Society Reviews 44, 5031-5039.

Yoo, S.-S. (2015): »3D-printed biological organs«. Expert Opinion on Therapeutic Patents 25(5), 507-511.

Yoo, S.-J.; Thabit, O.; Kim, E. K.; Ide, H.; Yim, D.; Dragulescu, A.; Seed, M.; Grosse-Wortmann, L. \& van Arsdell, G. (2016): »3D printing in medicine of congenital heart diseases«. 3D Printing in Medicine 2, 3. 
Yu, D. G.; Yang, X. L.; Huang, W. D.; Liu, J.; Wang, Y. G. \& Xu, H. (2007): »Tablets with material gradients fabricated by three-dimensional printing «. Journal of Pharmaceutical Sciences 96, 24462456.

Yu, D. G.; Zhu, L.-M.; Branford-White, C. J. \& Yang, X. L. (2008): »Three-dimensional printing in pharmaceutics«. Journal of Pharmaceutical Sciences 97, 3666-3690.

Zeng, K.; Pal, D.; Gong, H. J.; Patil, N. \& Stucker, B. (2014): »Comparison of 3DSIM thermal modelling of selective laser melting using new dynamic meshing method to ANSYS«. Materials Science and Technology 31, 945-956.

Ziemian, C.; Sharma, M. \& Ziemian, S. (2012): Anisotropic mechanical properties of ABS parts fabricated by fused deposition modelling. London: INTECH Open Access Publisher. 


Additive bio-manufacturing (bio-AM) is defined here as the use of 3D printing for medical purposes or non-therapeutic 'human enhancement', whether they involve the production of biological material or not. It includes any application for rehabilitating, supporting or augmenting biological functionality. The impacts of bio-AM are uncertain, and it is not clear which actions may be required to foster responsible development of the technology.

This study responds to these gaps in our knowledge by describing the state of the art and future development prospects, analysing their wide-ranging impacts - including social, ethical and economic aspects - and identifying key policy challenges along with options to respond to them. Key challenges are to avoid an incoherent, piecemeal adaptation of regulatory frameworks, to support responsible development that improves citizens' lives, and to foster public participation and citizen-driven innovation. The European RRI approach could provide a useful platform for responding to these challenges, emphasising inclusiveness and co-creation by a wide variety of stakeholder groups, including public participation.

This is a publication of the Scientific Foresight Unit (STOA)

EPRS | European Parliamentary Research Service, European Parliament

PE 614.571

ISBN 978-92-846-3148-3

doi: $10.2861 / 923327$

QA-01-18-636-EN-N

This document is prepared for, and addressed to, the Members and staff of the European Parliament as background material to assist them in their parliamentary work. The content of the document is the sole responsibility of its author(s) and any opinions expressed herein should not be taken to represent an official position of the Parliament. 